\title{
THE HEALTH IMPACT OF SEDENTARY BEHAVIOUR IN CHILDREN AND YOUTH
}

\begin{abstract}
Travis John Saunders
Thesis submitted to the

Faculty of Graduate and Postdoctoral Studies

in partial fulfillment of the requirements

for the Doctorate in Philosophy degree in Human Kinetics
\end{abstract}

School of Human Kinetics

Faculty of Health Sciences

University of Ottawa

Copyright (OTravis John Saunders, Ottawa, Canada 2013 


\begin{abstract}
Emerging evidence suggests that sedentary behaviour is independently associated with cardiometabolic disease risk in school-aged children and youth. This thesis includes 4 related studies in the pursuit of 2 objectives: 1) To determine the cross-sectional association of sedentary time, interruptions in sedentary time, sedentary bout length, and total movement variability with markers of cardiometabolic disease risk among children and youth, and 2) To examine the impact of 1-day of prolonged sedentary behaviour, with and without interruptions or structured physical activity, on markers of cardiometabolic disease risk, hunger, food intake and spontaneous physical activity levels in children and youth. In Study 1, we found that interruptions in sedentary time and short bouts of sedentary time were beneficially associated with clustered cardiometabolic disease risk in boys and girls aged 8-11 years, independent of total sedentary time, moderate-and-vigorous physical activity (MVPA), and other confounders (all $p<0.05$ ), while the opposite was true for screen based sedentary behaviours. In Study 2, we found that movement variability (minute-to-minute changes in movement intensity) was negatively associated with clustered cardiometabolic disease risk and systolic blood pressure independent of MVPA, sedentary time and other covariates in a representative sample of American children and youth aged 12-17 years (all $p<0.05$ ). In Studies 3 and 4, we found that prolonged sitting, with or without interruptions and structured MVPA did not result in acute changes in markers of cardiometabolic disease risk, nor subsequent ad libitum food intake or physical activity levels in healthy children aged 10-14 years (all $p \geq 0.05$ ). Taken together, the studies that make up this thesis suggest that optimal levels of cardiometabolic disease risk are most likely to be seen in children who limit their time engaging in screen-based sedentary behaviours, who frequently interrupt their sedentary time, and who have high levels of variability in their movement behaviours.
\end{abstract}




\section{Contributions}

The work in this thesis is my own, and I take full responsibility for its contents. Ethics applications were required for Studies 1, 3 and 4, and details of ethics approval are provided in Appendix A. A list of co-authors from the studies that make up this thesis can be found below. Details of the contributions of individual authors (including myself) to specific manuscripts can be found at the start of Chapters 2-6. At the time of final submission, 4 manuscripts in this thesis have been accepted for publication in the British Journal of Nutrition, the Canadian Journal of Diabetes, Metabolism and PLOS ONE. Both Metabolism and CJD are Elsevier journals, and therefore do not require a copyright form to be included in this thesis (See Appendix B). As the author I retain the copyright for manuscripts published in $B J N$, and PLOS ONE uses a Creative Commons Attribution license, therefore copyright forms are not required for these manuscripts either.

\section{Co-authors of works included in this thesis:}

Mark Tremblay (Chapters 2, 3, 4, 5 \& 6)

Jean-Philippe Chaput (Chapters 2, 3, 5 \& 6)

Gary Goldfield (Chapters 5 \& 6)

Rachel Colley (Chapters 5 \& 6)

Éric Doucet (Chapters 5 \& 6)

Glen Kenny (Chapters 5 \& 6)

Angelo Tremblay (Chapter 3)

Mélanie Henderson (Chapter 3)

Marie-Ève Mathieu (Chapter 3)

Jennifer O’Loughlin (Chapter 3)

Valerie Carson (Chapter 4) 


\section{Acknowledgements}

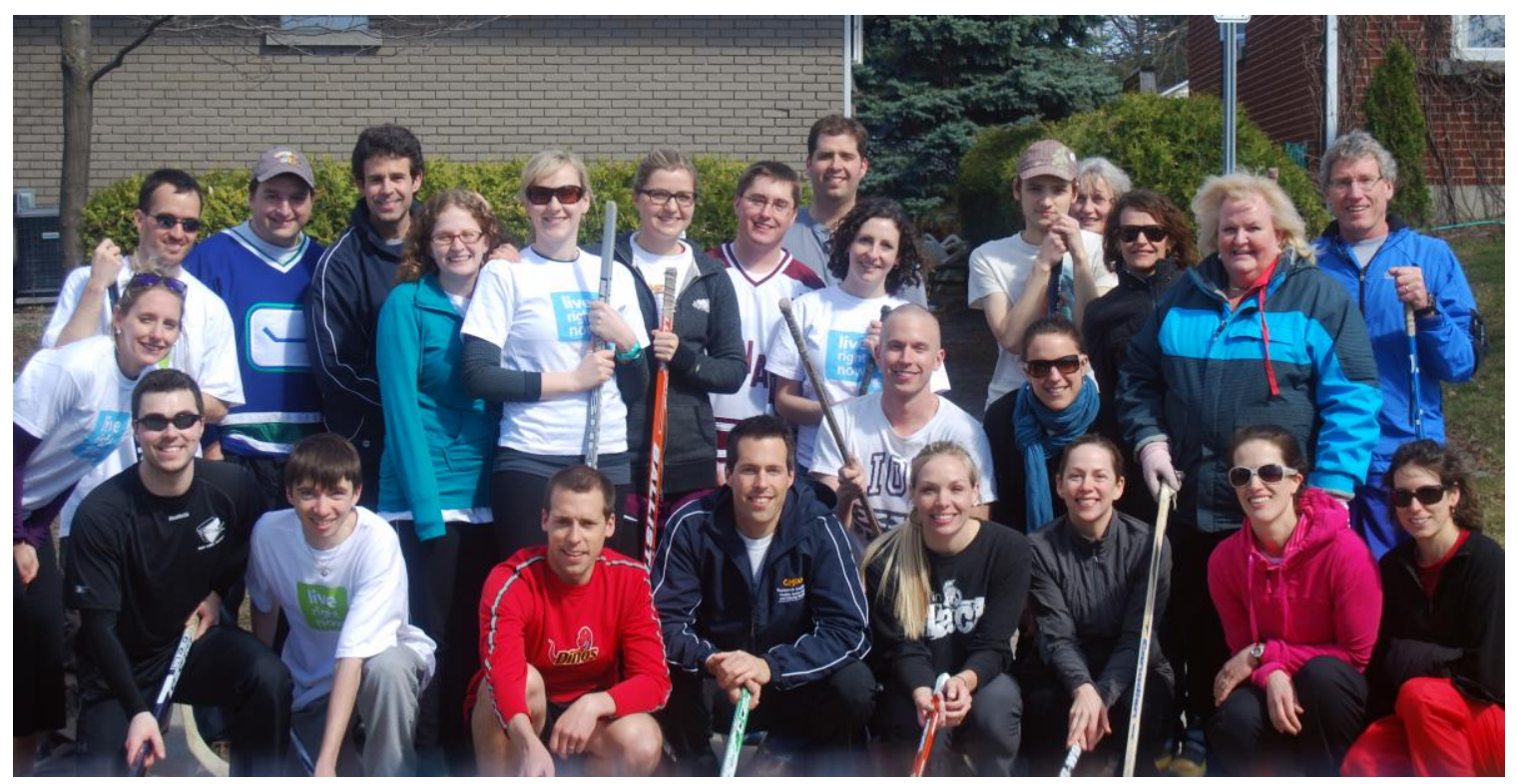

The HALO Research Group (and friends)

While I take full responsibility for the works that make up this thesis, none of the studies that follow would have been possible without the help of many friends and colleagues. I ask that the reader bear with me, as this section is one of the few opportunities that I will have to formally acknowledge and thank those individuals.

I would like to begin by thanking the many participants (and their families) who took part in the studies that make up this thesis. They volunteered a great deal of time, energy, and even blood, and without them none of these studies would have been possible. Not only that, but they helped make the long hours of data collection enjoyable, for which I am extremely grateful.

I would also like to sincerely thank my PhD supervisor Mark Tremblay. It is hard to find words to describe how much I appreciate all he has done for me as a supervisor and mentor. I came to work with Mark at a time when I was uncertain whether I wanted to continue with grad school. 
However, after just a few months working in the Healthy Active Living and Obesity Research Group that he oversees, my decision to pursue a PhD was both easy and obvious. Mark has been fiercely supportive of myself and my labmates, and has consistently provided me with rapid, detailed and (when necessary) blunt feedback throughout my PhD. I don't know that many graduate students are excited to go to work in the morning, but thanks to Mark (and the fantastic group he has assembled), I've always been able to look forward to work in "The Hub". Given the experience I have had working with Mark and the rest of the HALO team, I would also like to thank Michelle Lafrance, Doug Willms, and my uncle Patrick Flanagan for directly and/or indirectly sending me in this direction.

I would also like to sincerely thank Drs Gary "Gold Standard” Goldfield and Glen Kenny for sitting on my thesis committee, and for going far above and beyond the requirements in order to help me in my training and research. Gary has written me more reference letters than should be asked of anyone, been extremely supportive of my thesis projects, and has gone out of his way to include me in several productive side projects. Just as importantly, not since Chris Chelios has the (road) hockey world been blessed with such an ageless defenseman. For his part, Glen took time to mentor my teaching practicum, and has provided excellent advice in preparation for my comprehensive exam and thesis proposal, as well as the manuscripts included in this thesis. I have been very lucky to have the two of them on my thesis committee.

Although not officially part of my thesis committee, aside from Mark no one has had more impact on my PhD research than Dr Jean-Philippe Chaput. It was JP's Young Investigator Award that funded Studies 3 and 4 of this thesis, and it was his colleagues in Quebec that gave us access to the data used in Study 1. He has been an excellent colleague throughout, providing rapid and helpful feedback and support, and including me on a wide range of side projects. His 
productivity and work/life balance have also served as a model for myself and the other graduate students in the HALO lab.

I must also thank (fellow New Brunswicker!) Dr Éric Doucet for sitting on my comprehensive exam committee, and for welcoming me into his lab for my data collection. Studies 3 and 4 of this thesis would not have been possible without his help and support, and it has been a pleasure working with him throughout the thesis. I also appreciate the time and effort he has put into the graduate program in the School of Human Kinetics, first as a member of the Grad Committee and now as Director of Graduate Studies. Thanks also to the many wonderful people in his lab, including Ann Beninato, Isabelle Laforest, and Marie-Ève Riou, without whom data collection would have been impossible (and much less pleasant).

In addition to the aforementioned colleagues, I would also like to thank all of the collaborators who contributed to the manuscripts included in this thesis, as well as the side projects that I have been involved with over the years. They have all been extremely giving with their time, helpful in their feedback, and patient in responding to my questions (Drs Rachel Colley and Val Carson were especially helpful in this regard).

I would also like to thank all the members of the HALO Research Group at the Children's Hospital of Eastern Ontario Research Institute. I cannot imagine a better group of people to spend your days with. I will have especially fond memories of our regular games of HALO Hockey, and am pessimistic about ever finding another job that will allow me to play road hockey during my lunch hour multiple times every week. Particular thanks go to Helene Sinclair, Michelle Takacs, and Tina Hutchinson, the three excellent administrators who have helped keep HALO on track during my time with the group. I don't think anyone could put more into their 
job than Helene Sinclair, and it's been a pleasure to work with her these last few years.

Additional thanks also go to Niko Tzakis, Mike Borghese, Dr Zach Ferraro, Richard Larouche, Joel Barnes, Charles Boyer, and Allana Leblanc for their help with data collection and to Dr Kristi Adamo for always taking time from her busy schedule to help me navigate the research process. Thanks also to former HALOite Dr Meghann Lloyd for getting me involved in the CAPL study early in my time at HALO, and for Dr Pat Longmuir for keeping me involved since joining the group.

In addition to the folks at HALO, I have also been helped by a number of excellent staff members at the Children's Hospital of Eastern Ontario Research Institute. Thanks to Kim Allen and Sharon Haig for their unending patience and help with multiple finance and ethics applications, Dr Nick Barrowman and Kathryn Williams for their help with statistical questions, Dr Margaret Sampson for her expertise with systematic reviews, and Dr Catherine Pound for serving as the physician for studies 3 and 4 in this thesis.

I would be remiss if I did not also thank and acknowledge the funding agencies that have made my thesis possible. My PhD has been funded by Doctoral Research Awards from the Canadian Institutes of Health Research and the Canadian Diabetes Association, as well as an Excellence Scholarship from the University of Ottawa. Studies 3 and 4 in this thesis were also funded by a Young Investigator Award from the CHEO Research Institute to Dr JP Chaput.

Last, but certainly not least, I would like to thank my family for all the love and support that they have provided throughout graduate school, and life in general. Mom, Dad and my sister Katie have always been incredibly supportive, with everything from hockey to juggling to graduate studies, and I am very proud to be a member of their family. No one has contributed more to this 
PhD than my wife Daun, who I met shortly after entering graduate school in late 2006 . Thanks to her (and her alone) I've been able to maintain a sustainable level of work/life balance throughout my $\mathrm{PhD}$. She has made my PhD (and more importantly, life) a wonderful experience, and I can't thank her enough for her love and support during the past 4 years (and the 2 before that). I would also like to thank Daun's parents and the rest of the Lynch/Newland/Montgomery clan for welcoming me into their family, which has added so much to our time in Ottawa. Special thanks to Daun's stepfather, Dr Hugh Montgomery, for lending the famous wheelchair that was used to transport participants to and from the washroom in Studies 3 and 4. 


\section{Prelude to Thesis}

While the health importance of physical activity has been accepted for decades, recent evidence suggests that sedentary behaviour (e.g. sitting, and activities done while sitting) may be a unique and deleterious risk factor for chronic disease, independent of physical activity and other established risk factors. However, while numerous reports suggest a link between sedentary behaviour and health in the pediatric age group, several important questions remain unanswered. The following thesis attempts to advance our understanding of the relationship among sedentary behaviour, physical activity and health in the pediatric age group through the use of literature reviews, cross-sectional analyses of large datasets, and a lab-based randomized crossover study.

In Chapter 1 I offer an overview of the rationale, objectives and hypotheses of the current thesis. In Chapter 2 I provide a comprehensive overview of research into the health impact of sedentary behaviour in the pediatric age group. Building on the work presented in Chapter 2, Chapter 3 examines the association of total sedentary time, sedentary bout length, and breaks in sedentary time with markers of cardiometabolic disease risk in large cohort of children with a family history of obesity. Chapter 4 examines similar relationships for a novel characteristic of human movement; total movement variability. Chapters 5 and 6 examine the acute impact of prolonged sitting on markers of cardiometabolic disease risk, physical activity and energy intake. Finally, Chapter 7 discusses the implications of the findings laid out in Chapters 3-6, as well as opportunities for future research. Related studies that provide context for the findings presented in this thesis, as well as supporting documentation, can be found in the Appendices. It is my hope that the research included in this thesis will improve our understanding of the health impact of sedentary behaviour, and aid in the prevention of chronic disease among children and youth. 


\section{Table of Contents}

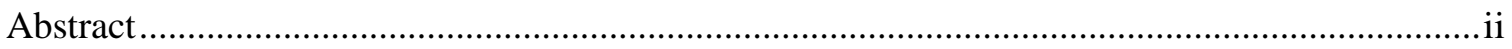

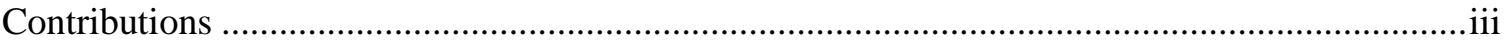

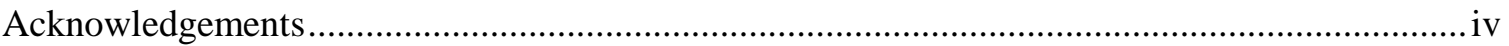

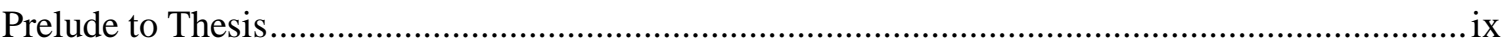

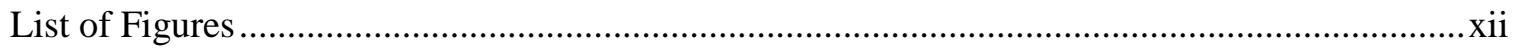

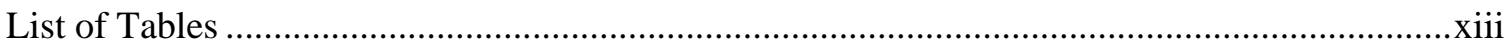

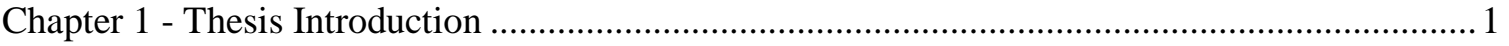

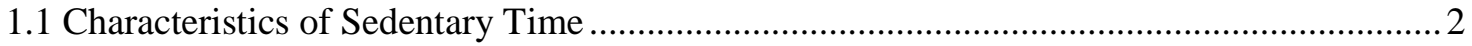

1.2 The impact of prolonged sitting on markers of cardiometabolic disease risk........................ 3

1.3 The impact of prolonged sitting on energy intake and physical activity ............................. 4

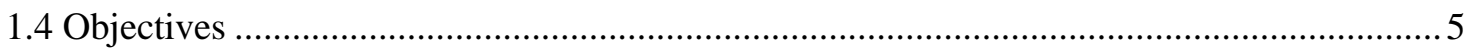

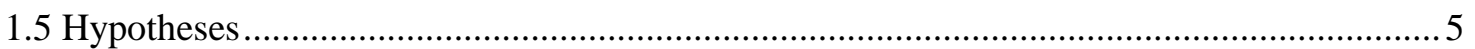

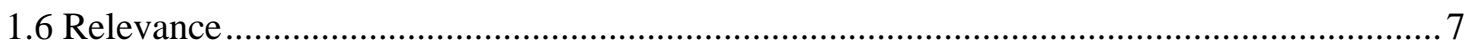

Chapter 2 - Literature Review: Sedentary behaviour as an emerging risk factor for

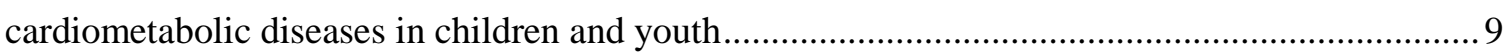

Chapter 3 - Study 1: Associations of sedentary behavior, sedentary bouts and breaks in sedentary time with cardiometabolic risk in children with a family history of obesity .................................. 43

Chapter 4 - Study 2: Movement variability is associated with clustered cardiometabolic disease

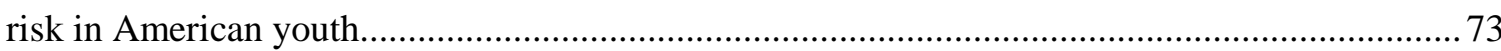

Chapter 5 - Study 3: Prolonged sitting and markers of cardiometabolic disease risk in children

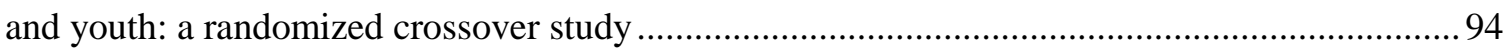

Chapter 6 - Study 4: Children and youth do not compensate for an imposed bout of prolonged sitting by reducing subsequent food intake or increasing physical activity: a randomized crossover study

Chapter 7 - General Discussion

7.1 Characteristics of sedentary behaviour: associations with health indicators

7.2 The impact of prolonged sedentary behaviour on markers of cardiometabolic disease risk,

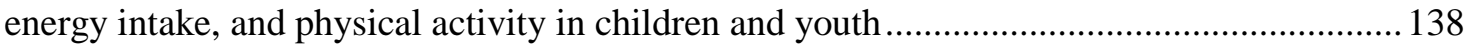

7.2.1 Does sitting per se have a negative impact on pediatric health?............................... 140

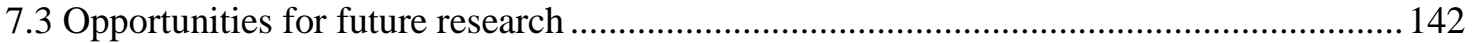

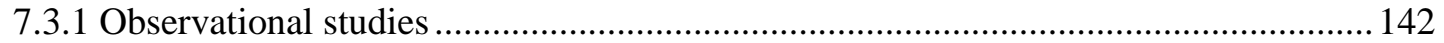

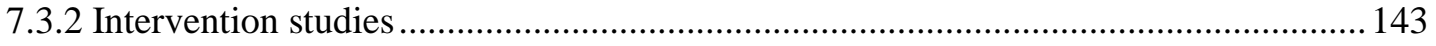




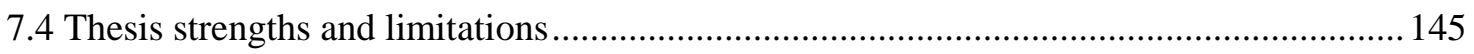

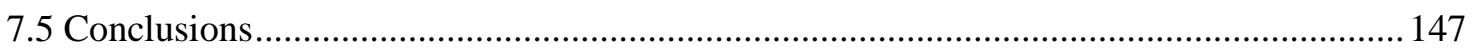

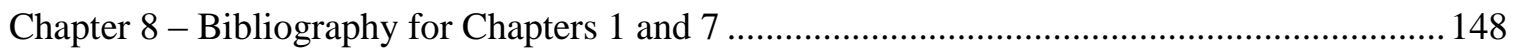

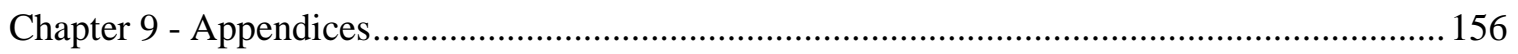

Appendix A : Ethics approval notices for thesis research projects .......................................... 157

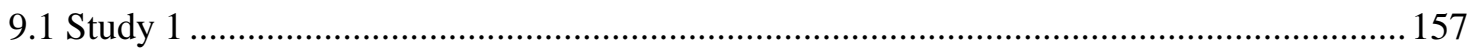

9.2 Study 2 (Data publically available, therefore no approval required) ................................ 159

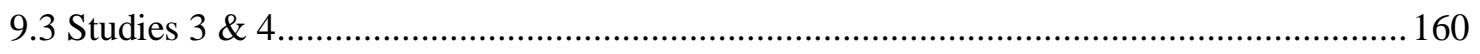

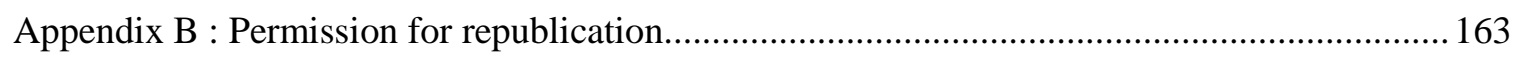

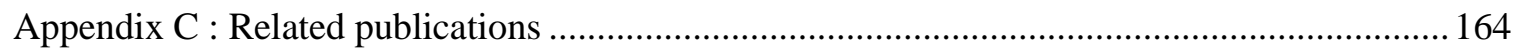

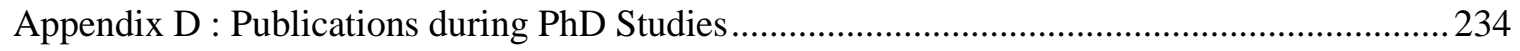

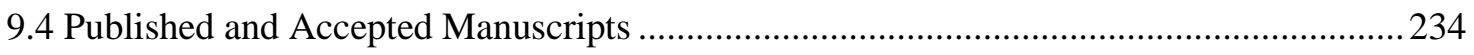

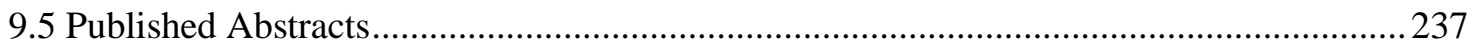




\section{List of Figures}

Figure 1.1 Sedentary behaviour and physical activity according to energy expenditure................. 1

Figure 2.1 Sedentary behaviour and physical activity as distinct constructs............................... 12

Figure 2.2 Self-reported media exposure of American youth over time....................................... 16

Figure 4.1 Examples of high and low movement variability.................................................... 76

Figure 4.2 Clustered cardiometabolic disease risk across tertiles of movement variability. ......... 82

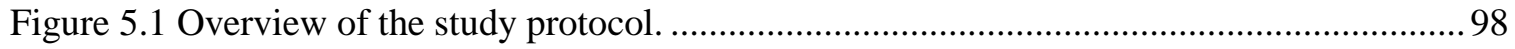

Figure 5.2 Insulin (A) and glucose (B) concentrations during 8 hours of prolonged sitting, with or

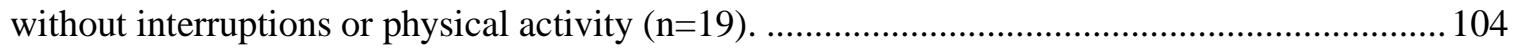

Figure 6.1 Overview of the study protocol (modified from Saunders et al. ${ }^{(16)}$ )......................... 118

Figure 6.2 Energy intake and estimated energy expenditure while in lab during a day of sitting with or without interruptions and structured physical activity................................................. 122

Figure 7.1 Examples of healthy and deleterious movement patterns....................................... 137 


\section{List of Tables}

Table 2.1 Comparison of reports examining the association between objectively measured sedentary time and markers of adiposity and cardiometabolic disease risk among children and

youth. 38

Table 3.1 Characteristics of study participants. 66

Table 3.2 Associations of sedentary behavior and physical activity with markers of cardiometabolic risk in boys.

Table 3.3 Associations of sedentary behavior and physical activity with markers of cardiometabolic risk in girls 70

Table 4.1 Subject characteristics 91

Table 4.2 Physical activity and sedentary behavior across tertiles of movement variability .92

Table 4.3 Associations between movement variability and markers of cardiometabolic disease risk in participants aged 12-17 years 93

Table 5.1 Participant characteristics.

Table 5.2 Net incremental area under the curve (iAUC) values for biomarkers of cardiometabolic disease risk during 8 hours of prolonged sitting, with or without interruptions or physical activity $(\mathrm{n}=19)$

Table 6.1 Characteristics of study participants at baseline.

Table 6.2 Measures of sedentary behaviour, physical activity, hunger and caloric intake during time spent in lab engaging in prolonged sitting, with and without breaks and structured physical activity $(n=20)$

Table 6.3 Sedentary behaviour and physical activity levels in the 24-hours immediately following prolonged sitting with or without breaks and structured physical activity $(\mathrm{n}=20) \ldots \ldots \ldots \ldots \ldots \ldots \ldots . . . . . . . .132$

Table 7.1 Summary of thesis key findings and contributions to the literature............................. 134

Table 7.2 Healthy and deleterious movement profiles for children and youth. 136 


\section{Chapter 1 - Thesis Introduction}

While the pediatric health benefits of physical activity are well-established (1-3), emerging evidence suggests that sedentary behaviour (activities that involve sitting or reclining while expending $\leq 1.5$ metabolic equivalents(4)) may be independently associated with increased health risk among children and youth (5-12).

Sedentary behaviour = any waking activity with an energy expenditure $\leq 1.5 \mathrm{METs}$

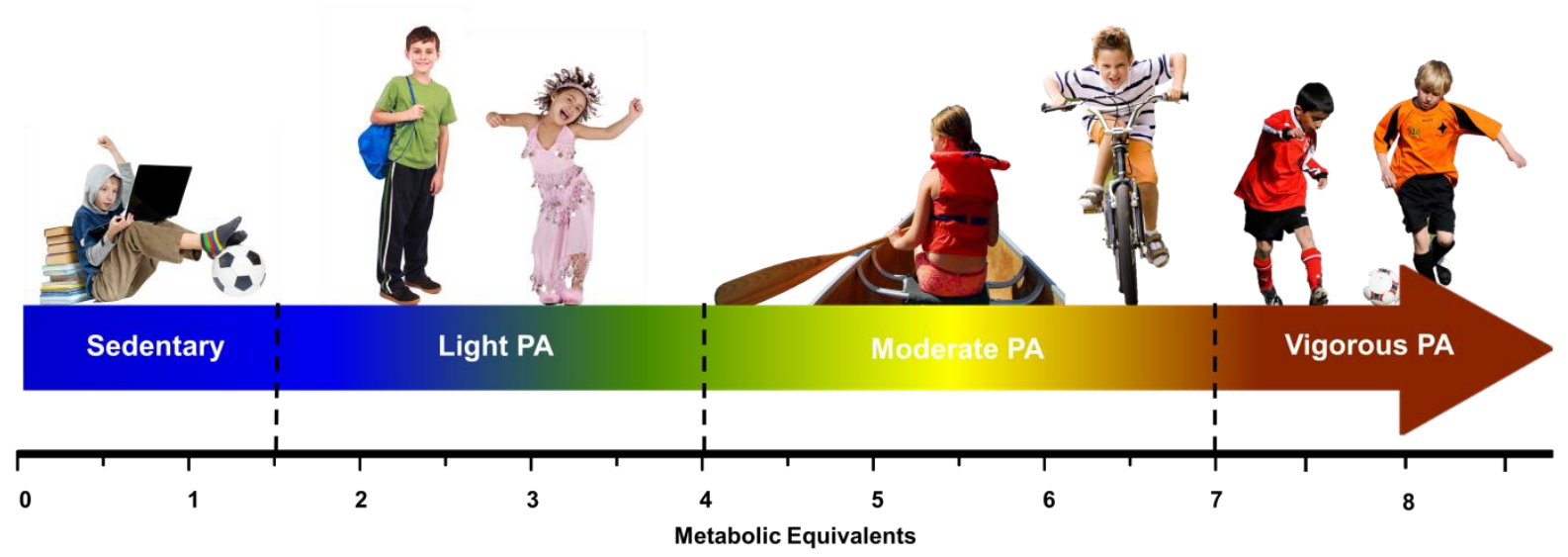

Figure 1.1 Sedentary behaviour and physical activity according to energy expenditure.

Despite a growing body of evidence suggesting that sedentary behaviour is associated with risk factors for cardiovascular disease and diabetes (hereafter referred to as cardiometabolic risk) in the pediatric population, several important questions remain. The purpose of the present thesis was to improve our understanding of the relationship between sedentary behaviour and cardiometabolic disease risk in school-aged children and youth. To do this, I have focused on 2 core areas of research: 
1. The cross-sectional relationship between characteristics of sedentary behaviour/human movement and markers of cardiometabolic disease risk.

2. The metabolic and behavioural impact of prolonged sitting.

\subsection{Characteristics of Sedentary Time}

While accumulating evidence suggests a relationship between total sedentary time and markers of cardiometabolic disease risk in the pediatric population, recent evidence in adults suggests that the manner in which sedentary time is accumulated may also have an important health impact. Interruptions in sedentary time (e.g. standing or walking for brief periods of time) are associated with reduced body weight, abdominal fat, and cardiometabolic disease risk independent of both total sedentary time and physical activity in adults $(13,14)$. Although a handful of studies have examined the association between breaks in sedentary time and markers of cardiometabolic disease risk in the pediatric population (15-18), none have been able to replicate the results observed in adults. Thus, at present it is unclear whether frequent interruptions in sedentary time are associated with reduced cardiometabolic disease risk among children and youth. However, it should be noted that past studies in this area have focused primarily on representative samples of children and youth $(15,16)$, who typically have low levels of cardiometabolic disease risk. It is possible that associations between characteristics of sedentary behaviour and cardiometabolic disease risk may be easier to detect among children with increased risk of cardiometabolic diseases, although this has not yet been investigated in any pediatric population.

Another unexamined aspect of human movement that may account for variation in cardiometabolic disease risk is total movement variability, defined as minute-to-minute changes in accelerometer counts per minute (CPM). It is also possible that variability per se may also be beneficial to health (19-23). For example, altering the output of mechanical ventilators to include random variations in breathing rate and volume results in improved oxygen saturation and organ 
function, when compared to ventilators that provide constant output (19,23). Further, for a given level of physical activity, individuals with high amounts of movement variability are likely to have lower levels of sedentary behaviour, and more frequent breaks in their sedentary time. To date no studies have examined the relationship between movement variability and cardiometabolic disease risk in any population.

\subsection{The impact of prolonged sitting on markers of cardiometabolic disease risk}

As mentioned above, there is an accumulating body of research in adults and children suggesting that excessive sedentary behaviour is associated with increased health risk. Further, evidence from both adults (24-26) and animal models (27) suggests that just a few hours of prolonged sedentary behaviour can result in dramatic changes in markers of cardiometabolic disease risk. Bey and Hamilton (27) have reported that just 6 hours of sedentary behaviour resulted in dramatic reductions in lipoprotein lipase activity in rat skeletal muscle, and that just one day of sedentary behaviour resulted in a $20 \%$ reduction in plasma HDL-cholesterol levels. Similarly, it has recently been reported that in comparison to a day that includes minimal sitting, a day of constant sitting reduced insulin action by $39 \%$ in healthy, recreationally active adults (24). In support of these findings, a recent systematic review from our group concluded that acute bouts of uninterrupted sedentary behaviour result in rapid and deleterious changes in insulin sensitivity, glucose tolerance, and lipid levels among adults (26).

Taken together, the above results suggest that as little as 1 day of uninterrupted sedentary behaviour may have a measureable and deleterious impact on markers of cardiometabolic disease risk. However, no study has examined the immediate cardiometabolic impact of a laboratorycontrolled bout of sedentary behaviour in children and youth. Therefore, despite evidence in both 
adult (24-26) and animal models (27), the acute influence of sedentary behaviour on markers of cardiometabolic disease risk in the pediatric population is unknown.

\subsection{The impact of prolonged sitting on energy intake and physical activity}

In addition to questions regarding the direct relationship between sedentary time and markers of cardiometabolic disease risk in youth, the relationship between sedentary behaviour and behavioural compensation (e.g. increases or decreases in physical activity or food intake associated with acute sedentary behaviour exposure) in the pediatric population is also unclear, and is another mechanism that may link excess levels of sedentary behaviour with increased health risk. For example, it has been reported that every one-hour increase in daily TV viewing among school-children is associated with an extra consumption of 167 calories per day (28). However, it is unclear whether this is due to factors related to chronic TV viewing (for example, exposure to advertisements for nutrient-poor foods (29), by distracting children from feelings of hunger and satiety (30)), or whether simply sitting for extended periods of time results in increased hunger and food intake in this population. It is worth noting that engaging in seated video game playing (31), has been reported to increase spontaneous food intake in adolescents, and a number of studies suggest that screen-based sedentary behaviours may lead to increased caloric consumption through a variety of mechanisms (32-34). However, despite the reported relationships between specific sedentary behaviours and subsequent food intake in adults, to date no study has investigated the impact of sitting per se, with or without breaks or structured physical activity, on hunger or food intake in children and youth.

As with hunger and food intake, to date no studies have examined the impact of prolonged sitting on subsequent levels of spontaneous physical activity. It has been suggested that physical activity may be centrally controlled by an "activitystat", such that individuals unconsciously increase or 
decrease their activity level to match energy intake or other internal cues (35). If true, this would suggest that children may increase their physical activity levels following a period of prolonged sitting. In support of this hypothesis, it has been reported that the introduction of structured physical activity may fail to increase (36), or may even reduce (37) total physical activity levels in the pediatric population, as a result of reductions in spontaneous physical activity and increases in sedentary behaviour. However, the impact of prolonged sitting on subsequent energy intake, physical activity and sedentary behaviour levels is presently unknown.

\subsection{Objectives}

The general objective of this thesis was to improve our understanding of the relationship between sedentary behaviour and markers of health in children and youth. In particular, it focused on 2 broad objectives that address the key gap areas described previously.

1. To determine the cross-sectional association of sedentary time, interruptions in sedentary time, sedentary bout length, and total movement variability with markers of cardiometabolic disease risk among children and youth.

2. To examine the impact of 1-day of prolonged sedentary behaviour, with and without interruptions or structured physical activity, on markers of cardiometabolic disease risk, hunger, food intake and spontaneous physical activity levels in children and youth.

\subsection{Hypotheses}

The general hypothesis of this thesis was that sedentary time would be positively associated with increased health risk among children and youth in both cross-sectional and intervention analyses. The specific hypotheses, and the individual studies that they accompany, are found below. 
Study 1. We hypothesized that markers of cardiometabolic disease risk would be negatively associated with interruptions in sedentary time and positively associated with long bouts of uninterrupted sedentary time in cross-sectional analyses. We hypothesized that these relationships would be independent of total sedentary behaviour and physical activity levels.

Study 2. We hypothesized that markers of cardiometabolic disease risk would be negatively associated with total movement variability (defined as the minute-to-minute variation in movement intensity as assessed using accelerometer counts per minute) in children and youth. We hypothesized that this relationship would be independent of total sedentary time and physical activity levels.

Study 3. We hypothesized that a day which included short breaks in sedentary time or structured physical activity would result in significantly lower levels of cardiometabolic disease risk (defined as insulin, glucose and lipid area-under the curve), in healthy children and youth, as compared to a day of prolonged sitting. Further, we hypothesized that a day which included both interruptions in sedentary time and structured physical activity would result in greater reductions in markers of cardiometabolic disease risk than a day which included interruptions in sedentary time but no structured physical activity.

Study 4. We hypothesized that a day which included short breaks in sedentary time or structured physical activity would not influence subsequent levels of energy intake and hunger in children and youth, when compared to a day of prolonged sitting. We further hypothesized that participants would compensate for a day of prolonged 
sitting by reducing their level of sedentary time, and increasing their level of physical activity, in the subsequent 24 hours.

\subsection{Relevance}

At present it is unclear whether characteristics of sedentary time or total movement variability are associated with markers of cardiometabolic disease risk in children and youth. It is also unknown whether prolonged sitting results in acute changes in markers of cardiometabolic disease risk or behavioural compensation. These critical gaps in knowledge are extremely important from a public health perspective, as recent evidence suggests that Canadian children spend roughly 8 hours per day - more than half of their waking hours - sitting (38-40). More than $70 \%$ of the average school day, including physical education, is sedentary (39), while the average Canadian child reports accumulating more than 6 hours per day of screen time during their discretionary leisure time (40). Given the ubiquity of sitting in the Canadian pediatric population, it is critical that we improve our understanding of the relationship between sedentary behaviour and health in pediatric populations.

The knowledge gaps described above limit our understanding of the health impact of sedentary behaviour in children and youth, and preclude the development of maximally efficacious interventions in this age group. Although cross-sectional evidence in adults suggests that characteristics of sedentary time may account for cardiometabolic disease risk beyond that explained by total sedentary time alone, these findings have yet to be replicated in children and youth. Similarly, while accumulating evidence suggests that sedentary behaviour is associated with increased health risk in children, it is unknown whether a single session of lab-controlled sedentary behaviour has a measurable metabolic impact in the pediatric population, or whether characteristics of sedentary time influence this impact. The studies contained within this thesis 
are an attempt to address these knowledge gaps, and will assist in the development of improved clinical and public health interventions targeting chronic disease risk in this age group. 


\title{
Chapter 2 - Literature Review: Sedentary behaviour as an emerging risk factor for cardiometabolic diseases in children and youth
}

\section{The following article is has been accepted for publication in the Canadian Journal of Diabetes, and has been formatted according to their requirements}

\author{
Authors: Travis J Saunders ${ }^{1,2}$, Jean-Philippe Chaput ${ }^{1,2}$, Mark S Tremblay ${ }^{1,2}$ \\ Affiliations: \\ ${ }^{1}$ Healthy Active Living and Obesity Research Group, Children's Hospital of Eastern Ontario \\ Research Institute, Ottawa, Ontario, Canada \\ ${ }^{2}$ School of Human Kinetics, University of Ottawa, Ottawa, Ontario, Canada

\section{Author Contributions} \\ The article was conceived and designed by TJS, JPC and MST. TJS wrote the initial draft of the \\ manuscript, while MST and JPC provided critical edits and additions. All authors approved the \\ final manuscript.
}

\section{Acknowledgements}

TJS is supported by a Doctoral Research Award from the Canadian Institutes of Health Research, a Doctoral Studentship Award from the Canadian Diabetes Association, and an Excellence Scholarship from the University of Ottawa. JPC holds a Junior Research Chair in Healthy Active Living and Obesity Research. Some of the research reported in this review was supported by a Young Investigator's Award to JPC from the Children's Hospital of Eastern Ontario Research Institute. 


\begin{abstract}
Sedentary behaviour (e.g. TV viewing, seated video game playing, prolonged sitting) has recently emerged as a distinct risk factor for cardiometabolic diseases among children and youth. This narrative review provides an overview of recent evidence in this area, and highlights research gaps. Current evidence suggests that North American children and youth spend between 40 and $60 \%$ of their waking hours engaging in sedentary pursuits. Although data are lacking on temporal trends of objectively measured sedentary time, self-reported sedentary behaviours have increased over the last half-century, with a rapid increase since the late 1990's. Excessive sedentary behaviour has been found to have independent and deleterious associations with markers of adiposity and cardiometabolic disease risk. These associations are especially consistent for screen-based sedentary behaviours (TV viewing, computer games, etc), with more conflicting findings observed for overall sedentary time. The above associations are possibly mediated by the influence of screen-based sedentary behaviours on energy intake. Although excessive sitting has been reported to have adverse acute and chronic metabolic impacts among adults, research on children is lacking. Research is particularly needed to investigate the impact of characteristics of sedentary behaviour (i.e. type/context, sedentary bout length, breaks in sedentary time, etc), as well as interventions that examine the health and behavioural impact of sitting per se.
\end{abstract}

Keywords: Sedentary behaviour, pediatrics, cardiometabolic disease 


\section{Introduction}

It is well established that high levels of physical activity are associated with reduced health risk in children and youth (1-3). Physical activity exhibits a dose-response relationship with health indicators in the pediatric population, and even modest amounts of physical activity can result in improved health for those at greatest risk (1). However, in addition to the consistent association between physical activity and health in the pediatric population, accumulating evidence suggests that the amount of time children and youth spend engaging in sedentary behaviours (i.e. activities that involve sitting or reclining while expending $\leq 1.5$ metabolic equivalents (4)) may be associated with increased cardiometabolic disease risk independent of other factors such as physical activity and abdominal obesity (5-12). In response to this new research, Canada has recently created pediatric sedentary behaviour guidelines, which are separate from (but complementary to) physical activity guidelines for this age group (11). These guidelines recommend that school-aged children and youth accumulate no more than 2 hours of recreational screen time each day, and that they also limit periods of prolonged sitting and motorized transport (11). Although a number of recent narrative reviews have examined the health impact of sedentary behaviour in adults (13-17), there is a lack of such a review in the pediatric population. Thus, this article aims to provide a comprehensive overview of the available evidence concerning sedentary behaviour and markers of cardiometabolic disease risk in school-aged children and youth. 
What is sedentary behaviour?

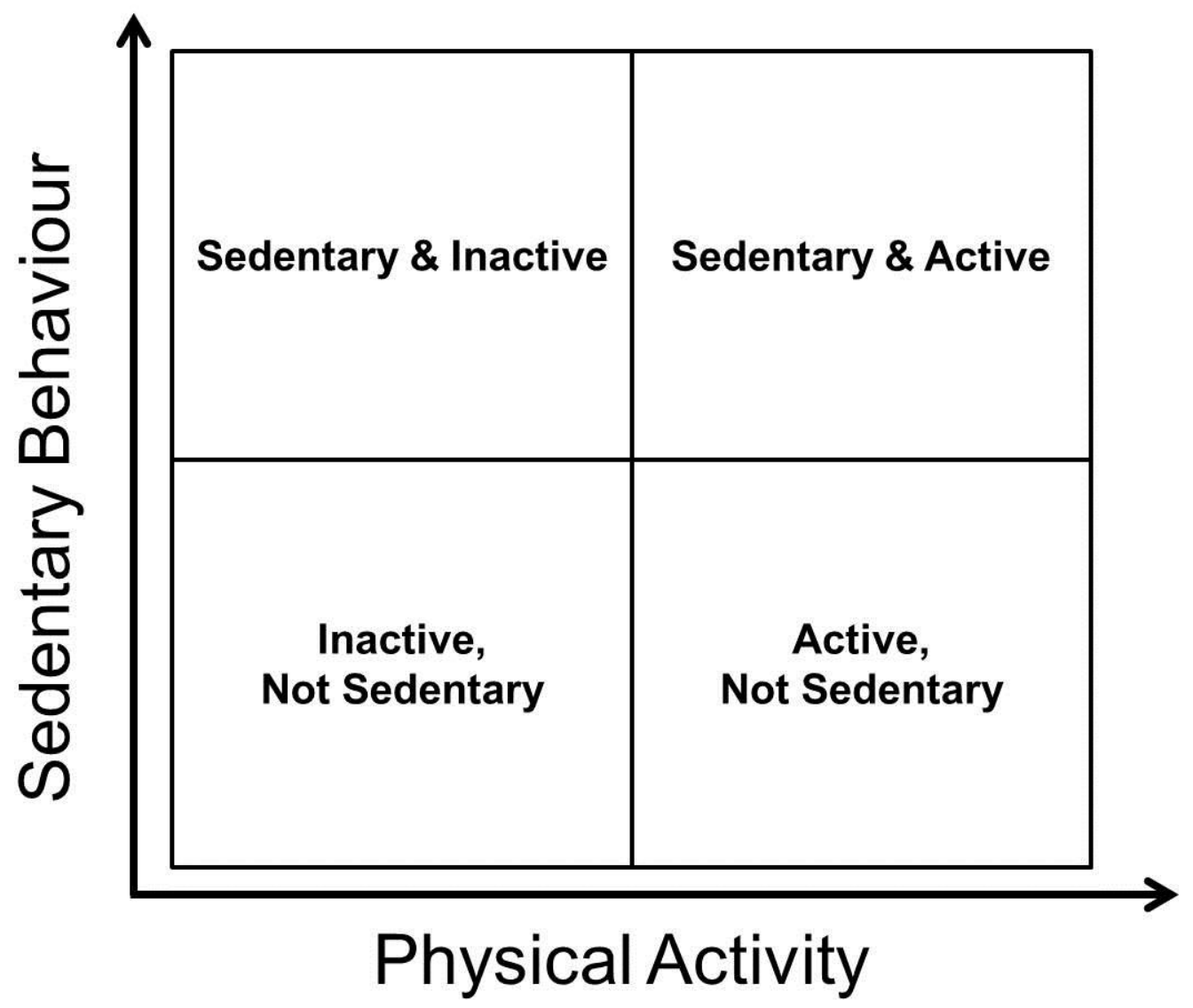

Figure 2.1 Sedentary behaviour and physical activity as distinct constructs.

What is sedentary behaviour?

The meaning of the word "sedentary" has evolved rapidly in recent years (18). Although the Latin root of the word sedentary literally means "to sit" (15), the term has historically been used by health researchers to refer to an individual who is not sufficiently physically active (4). Similarly, the phrase "sedentary lifestyle" has typically been used to refer to a lifestyle which includes little or no physical activity (19). It has therefore been relatively common for researchers to refer to individuals as "sedentary" due to their lack of physical activity, rather than the amount of time they spend sitting. However, recent evidence suggests that sitting too much and 
exercising too little are separate and distinct risk factors for chronic diseases including cancer, cardiovascular disease, and diabetes $(15,16,20,21)$. Further, an individual can easily meet physical activity guidelines while spending the vast majority of their day engaging in seated activities, or vice versa (see Figure 2.1). As a result, it has been proposed that the term "sedentary" should be used only to refer to activities which are defined by both a seated or reclining posture, and an energy expenditure at or near resting levels (4). Therefore, in this review the term sedentary will be used to specifically refer to waking behaviours characterized by energy expenditure $\leq 1.5$ METs while in a sitting or reclining posture (4). In contrast, the term "inactive" will be used to refer to an individual who is not sufficiently physically active (e.g. not meeting physical activity guidelines).

\section{How is pediatric sedentary behaviour measured?}

As with physical activity, sedentary behaviour can be assessed using a variety of self- and proxyreport questionnaires, or by direct measurement tools $(15,22,23)$. Self- and proxy-report tools typically take one of two approaches: 1) asking children or their parents to estimate the amount of time that they spend engaging in common sedentary behaviours (e.g. watching television, using a computer, playing passive video games, driving in a car, etc) which may be reflective of total sedentary time, or 2) asking them to estimate the amount of total time that they spend sitting on a daily basis. These tools are attractive because they are inexpensive and result in data that are relatively simple to analyze, while providing information related to specific modalities or contexts of sedentary behaviour (e.g. television viewing vs. reading). A recent systematic review suggests that self- and proxy-report tools generally display acceptable reliability and validity in assessing sedentary behaviour (22). However, these measures have a number of limitations. First and foremost, they are known to be limited by high levels of error and recall bias (23-26). Further, no single sedentary activity is representative of an individual's total sedentary behaviour 
profile $(23,27,28)$, which can pose an issue when data collection focuses on a limited number of sedentary behaviour modalities.

In contrast to self-report tools, accelerometers and inclinometers allow for the direct measurement of sedentary behaviour in childhood $(15,22,23)$. Accelerometers assess the number of movement "counts" in a given time period, and their use has increased rapidly in recent years (29). A variety of thresholds have been proposed to distinguish between sedentary behaviour and lightintensity physical activity, with a threshold of 100 counts per minute (CPM) being shown to have high sensitivity and specificity for the measurement of sedentary behaviour in pediatric populations using both Actigraph (Actigraph, Pensacola, USA) and Actical (Philips Respironics, Andover, USA) accelerometers (22,30-36). Accelerometers can also be used to assess the frequency of breaks in sedentary time and the duration of sedentary bouts, neither of which can be determined easily via self-report tools (37-39). However, a key limitation of accelerometers is their inability to distinguish between sitting and stationary standing (40), and the lack of information regarding the modality of sedentary behaviour (e.g. TV viewing vs. reading). Inclinometers such as the activPAL (PAL Technologies, Glasgow, UK) have been reported to be more accurate than accelerometers in differentiating between sitting and standing $(40,41)$, with Aminian and Hinckson reporting that the activPAL was able to perfectly distinguish between the two postures in healthy elementary school children (41). As with accelerometers, however, inclinometers are unable to provide information on the modality of sedentary behaviour, and have been used far less frequently. As a result of the limitations of both self-report and direct measurement tools, researchers have therefore advocated for the concurrent use of both strategies whenever possible $(22,23)$.

\section{Prevalence of sedentary behaviour in the pediatric population}


Available evidence suggests that children and youth in developed nations currently spend 40-60\% of their waking hours engaging in sedentary pursuits. Colley et al. used accelerometers to assess total sedentary time in a representative sample of 1,608 Canadians between the ages of 6 and 19 years $(37,42)$. They estimate that girls and boys respectively accumulate 7.4 and 8.5 hours of daily sedentary time, roughly half of which is accumulated during school hours $(37,42)$.

Sedentary time also tends to increase with age; children under 11 years averaged approximately 1.3 hours less daily sedentary time than those aged 11-14 years, and roughly 2 hours less than those aged 15-19 years (42). Similar levels and trends for accelerometer-derived sedentary behaviour have been reported in cross-sectional examinations of American (43) and European (44) children and youth.

The above-mentioned findings are also supported by longitudinal studies, which suggest that both screen time and total sedentary time increase with age $(45,46)$. For example, a longitudinal study of 759 Vietnamese students observed that boys and girls increased their daily sedentary time by more than 1 hour between the ages of 13 and 16 years (45). Similarly, Brodersen et al. found that self-reported screen time increased by more than 2.5 hours/week during a 5 -year period in a study of 5,863 British adolescents (46). It is worth noting that the frequency of breaks in sedentary time also appears to decrease with age; a longitudinal study of roughly 500 children found a decrease of approximately 2 breaks/hour/year from age 5 to age 15 (47). These findings suggest that children become more sedentary with age, and also accumulate their sedentary time in increasingly prolonged bouts. 
Temporal trends in sedentary behaviour among children and youth

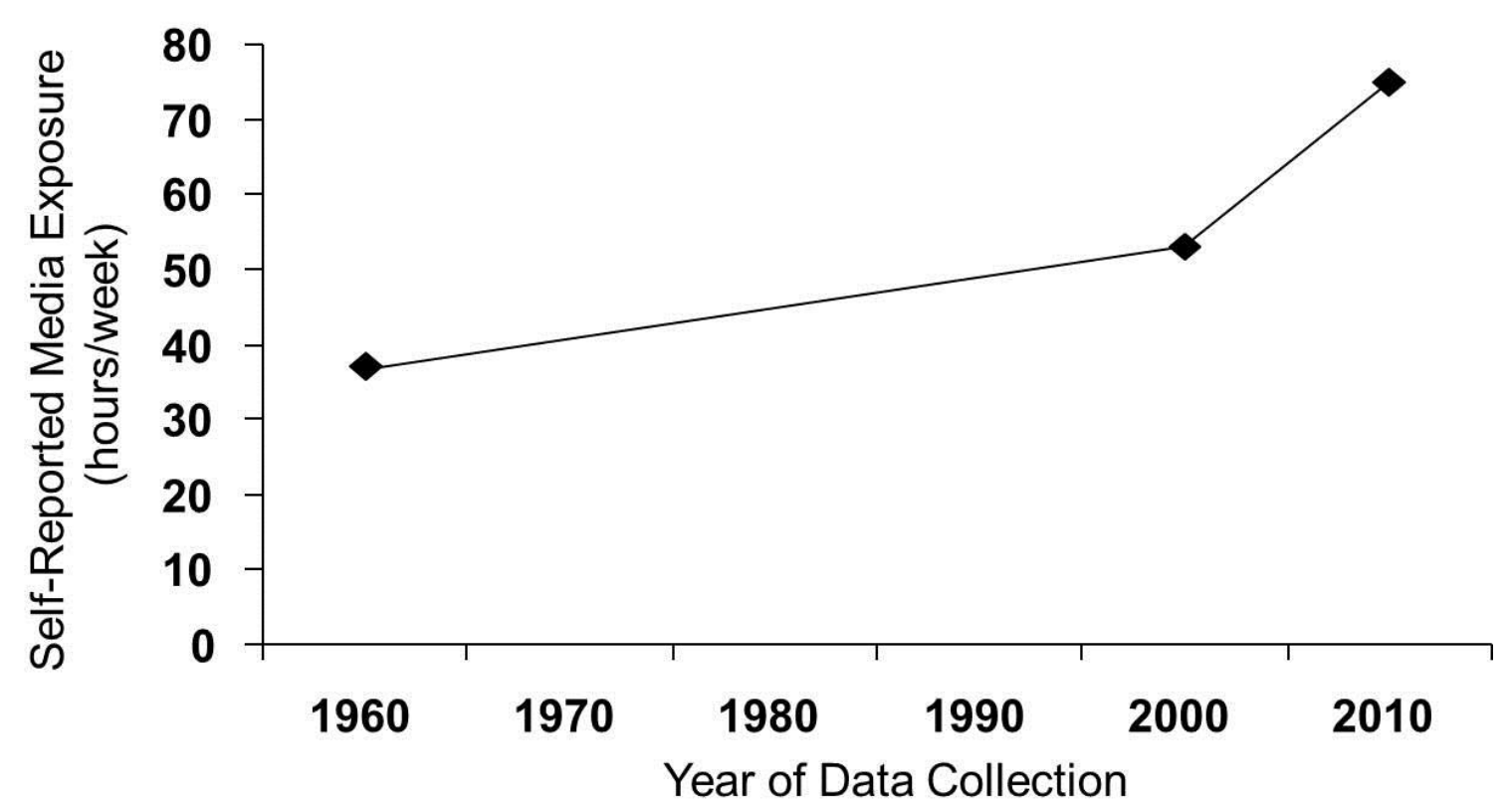

Figure 2.2 Self-reported media exposure of American youth over time.

Data from Schramm et al. (45) and Rideout et al. (46). Data have not been adjusted for multi-tasking (e.g. engaging with multiple media simultaneously).

Given the relatively recent introduction of accelerometry in population-based research, it is difficult to assess temporal trends in objectively measured sedentary time. However, selfreported media use (including TV, radio, audio, reading, etc) appears to have increased since the 1960's, with rapid increases observed in the past decade. Schramm et al. (48) reported that American children in grades 6 and 12 averaged roughly 37 hours/week of total media exposure in 1961. In contrast, recent evidence from the Kaiser Family Foundation reported that American children between the ages of 8 and 18 years averaged 53 hours/week of total media exposure in 1999, and 75 hours in 2009 (49) (Figure 2.2). After adjusting for multi-tasking (e.g. engaging with two forms of media simultaneously), the average American youth currently spends 54 hours engaging with media each week (49). The same report estimated that total media use increased by 1.5 hours/day among White American children between 1999 and 2009, and by more than 3 hours/day among Black and Hispanic children over the same period (49). A nationally 
representative study of 52,000 children and youth produced a similar estimate of total daily screen time among contemporary Canadian students (50), and temporal increases in self-reported screen time have also been reported in Czech girls (but not boys) (51) and Chinese children and youth $(52,53)$ during the late 1990's and early 2000's, although reductions in total screen time have been reported in Norwegian children (54) and Czech boys (51). Consequently, it is not surprising that the majority of children in developed nations currently exceed pediatric screen time recommendations $(11,55)$.

Along with the reported increases in total screen time, there has also been a shift away from TV viewing, and towards increased computer and video game use in recent decades $(49,51,56,57)$. In a study of Czech children between 1998 and 2008, the percent of sedentary time accounted for by TV viewing decreased from 17 to $12 \%$ among girls, and from 24 to $15 \%$ among boys (51). During the same period, the proportion of sedentary time accounted for by computer use more than doubled in both sexes (51). Finally, evidence suggests that sedentary modes of transportation (e.g. driving) have also increased dramatically since the 1960's throughout the Western world (58-60). Taken together, the above evidence suggests that the volume of total daily sedentary time has likely increased in the past 50 years, with computer and video game use playing a larger role in recent years.

\section{Sedentary time and markers of adiposity in children and youth}

A recent systematic review by Tremblay et al. (61) examined the relationship between sedentary behaviour (typically assessed via self- or proxy-reported screen time) and adiposity in 170 separate studies of school-aged children. Among 119 cross-sectional studies, 94 observed positive associations between sedentary behaviour and markers of adiposity. Further, the risk of being identified as obese increased in a dose-response manner with sedentary time. For example, in a sample of 461 Mexican children and youth, Hernández and colleagues observed that the odds 
of being classified as obese increased by $12 \%$ for every hour of self-reported television viewing (62). These cross-sectional findings are also supported by longitudinal evidence $(61,63,64)$.

Mitchell et al. (64) showed that objectively measured sedentary time was independently associated with increased weight gain between 9 and 15 years of age among children at the $50^{\text {th }}$, $75^{\text {th }}$ and $90^{\text {th }}$ body weight percentiles, independent of other covariates including physical activity, sleep, and diet.

Finally, evidence from randomized controlled trials demonstrates that reductions in sedentary time may result in reductions in adiposity $(61,65,66)$. For example, Robinson reported that elementary school children who were randomized to receive an intervention aimed at reducing screen time experienced a $0.45 \mathrm{~kg} / \mathrm{m}^{2}$ reduction in body mass index $(\mathrm{BMI})$ and a $2.30 \mathrm{~cm}$ reduction in waist circumference when compared to control students over a 6 month period (65). These findings are supported by a recent systematic review and meta-analysis, which concluded that interventions that reduce sedentary behaviour in children result in a mean decrease in BMI of $0.89 \mathrm{~kg} / \mathrm{m}^{2}$ (61). These results suggest that sedentary behaviour (especially screen time) has an independent and causal influence on the risk of excess weight gain and obesity in the pediatric age group $(61,67)$.

\section{Sedentary time and markers of cardiometabolic disease risk in children and youth}

Although it has been the focus of less research than adiposity, emerging evidence suggests that sedentary behaviour is also independently associated with other markers of cardiometabolic disease risk in children and youth $(5,8-10,12,61,68-71)$. Goldfield et al. have recently reported that television viewing and video game playing are independently associated with risk factors for diabetes and cardiovascular disease, respectively, independent of physical activity in overweight and obese adolescents $(9,10)$. Similarly, Kriska et al. (71) observed that in comparison to obese youth without diabetes, those recently diagnosed with diabetes accumulated roughly 1 additional 
hour of objectively measured sedentary time each day. These results are also supported by a recent report by Wennberg et al. (72), who found that self-reported TV viewing at age 16 is prospectively associated with the risk of metabolic syndrome at age 43 years. Participants who reported watching "several TV shows a day" at baseline had twice the odds of having metabolic syndrome at follow-up, independent of physical activity, socioeconomic status, and family history of diabetes. Associations were also seen for individual metabolic syndrome components including central obesity, lipids, and blood pressure (72). As with adiposity, these findings suggest that sedentary behaviour (typically measured as self-reported screen time) is independently associated with increased cardiometabolic disease risk in the pediatric population.

The role of sedentary behaviour modality: screen based vs. non-screen based sedentary time In the 5 year span between 2005 and 2010, the number of investigations assessing sedentary behaviour using objective measures doubled (29). As the volume of studies using both objective measures of sedentary behaviour (which assess total sedentary time) and self-reported sedentary behaviour (which typically focus on specific sedentary behaviours like screen time) has increased, a surprising trend has become apparent in the literature. While self-reported screen time is consistently associated with increased adiposity and cardiometabolic disease risk in children and youth independent of physical activity levels (7-10,61,68,70,73-75), the relationship between objectively measured sedentary time and health indicators is far less clear. Of the numerous studies examining the relationship between objectively measured sedentary time and markers of adiposity and cardiometabolic disease risk in the pediatric population $(5,12,37,64,68,69,71,74-82)$, only a small number $(64,69,79)$ have detected associations which remained significant after adjustment for physical activity (Table 2.1). These findings raise questions about the health impact of sitting per se, in comparison to the impact of specific screenbased sedentary behaviours. 
The differences between self-reported screen time and directly measured total sedentary time are most apparent when examined using a single cohort. For example, Carson and Janssen observed that self-reported TV viewing was independently associated with clustered cardiometabolic disease risk in a nationally representative sample of American children and youth (70). In contrast, the authors observed no independent associations between accelerometer-derived sedentary time and markers of cardiometabolic risk in the same sample. Similar findings have also been reported in other cohorts $(68,73-75,77)$. Chaput and colleagues found that self-reported screen time (but not objectively measured total sedentary time) was independently associated with increased waist circumference and reduced HDL-cholesterol concentrations in a cohort of 536 children at-risk for obesity (68). Similarly, Martinez-Gomez et al. (75) reported that several biomarkers were independently and deleteriously associated with self-reported TV viewing, but not objectively measured sedentary time, in a group of Spanish adolescents.

Given the bias and error that are known to be associated with self-report measures $(24,26)$, it is somewhat surprising that self-reported screen time appears to be more closely associated with health indicators than an objective measure of total sedentary time such as accelerometry. It is not uncommon for studies to report levels of self-reported screen time that seem highly implausible $(26,83)$, a characteristic that has been observed in other forms of self-report data collection as well (84). For example, a recent study found that a group of highly active and highly sedentary 10-11 year old students self-reported an average of 13.9 hours per day of screen time, and another 5.9 hours of physical activity $(26,83)$. If this were true, it would leave only 4.2 hours each day for eating, sleeping, and attending school, which seems unlikely. It has also been noted that the association between self-reported and directly measured sedentary behaviour can be extremely small $(23,70)$. Carson and Janssen observed a correlation of just 0.08 between selfreported TV viewing and accelerometer-derived sedentary time in a nationally representative sample of American children and youth (70), suggesting little overlap between the two measures. 
There are a number of factors that could explain the differences observed between self- or proxyreported screen time and objectively measured sedentary time in children and youth. Self- and proxy-reports of sedentary behaviour typically only provide information on a single behaviour (a subset of total sedentary time), while objective measures of sedentary time provide a global measure of time spent sedentary. The two types of measures are therefore assessing different things (23). For this reason, self- and proxy-reported sedentary behaviour typically account for far less total time than do objective measures; Colley et al. found that parent-reported screen time was equivalent to only a third of total sedentary time assessed via accelerometry in a representative sample of Canadian children (23). Given the highly sedentary nature of contemporary life $(42,43,47,85,86)$, it has also been suggested that the weak associations seen with objective measures of sedentary behaviour and different health indicators in children and youth may be due to a lack of inter-individual variability (23). Further, it has been noted that a variety of methods have been used to process accelerometer data in the pediatric population, and this may have a significant impact on the results of individual studies, making it difficult to directly compare results from separate investigations $(29,79)$. For example, studies have excluded data as "non-wear" time when there are as few as $10(5,75)$, or as many as $100(79)$ consecutive minutes with accelerometer values of 0 CPM. Further, although an accelerometer threshold of $100 \mathrm{CPM}$ is used most commonly to identify sedentary behaviour, thresholds as high as $1100 \mathrm{CPM}$ have been used in studies examining the relationship between sedentary time and markers of adiposity and cardiometabolic risk in the pediatric age group (79). While the impact of such methodological issues is not certain, a recent report by Atkin et al. (79) suggests that discrepancies in sedentary thresholds (e.g. 100 CPM vs 1100 CPM) are likely to have a much larger impact than differences in non-wear time. Counter intuitively the same authors reported that higher thresholds, which therefore classify higher intensities of movement as sedentary time, result in stronger associations between sedentary time and markers of cardiometabolic disease 
risk in the pediatric population. Further research into the impact of such methodological issues, and techniques for comparing across studies employing different methodologies, is clearly warranted.

Finally, as discussed below, it is also possible that certain forms of sedentary behaviour (e.g. TV viewing and other forms of screen time) may disproportionately promote other unhealthy behaviours such as excess food intake, which may explain why they are more consistently associated with health risk in the pediatric population $(67,87)$. Taken together, these findings suggest that screen time (and especially TV viewing time (88)) may be more closely associated with markers of cardiometabolic disease risk than total objectively measured sedentary time in the pediatric population, and this reinforces the notion that researchers should collect data using both measures whenever possible $(22,23)$.

\section{Characteristics of sedentary behaviour: impact on health indicators}

In addition to the health impact of total sedentary time and specific sedentary behaviours (e.g. screen time), recent evidence in adults suggests that certain patterns of sedentary behaviour may also have an important health impact $(38,39,89,90)$. A recent systematic review (89) concluded that prolonged bouts of uninterrupted sedentary behavior have a rapid and deleterious impact on insulin sensitivity, glucose tolerance, and triglyceride levels in adults. Further, interruptions in sedentary time have been shown to be beneficially associated with body weight, abdominal fat, triglycerides and glucose metabolism in adults $(38,39,90)$. These findings have yet to be replicated in the pediatric population.

Carson and Janssen failed to detect any associations between breaks in sedentary time, sedentary bout length, and cardiometabolic disease risk in a nationally representative sample of 2,527 American children and youth after adjustment for potential confounders (70). Kwon et al. (47) 
also reported no association between breaks in sedentary time and fat mass in a sample of 544 boys and girls in the Iowa Bone Development Study, with similar findings being reported among children from the Pacific Islands (91). To our knowledge, only one study to date has reported an association between characteristics of sedentary time and health indicators in children and youth; Colley and colleagues found that prolonged bouts of sedentary behaviour (those lasting 80+ minutes) are positively associated with waist circumference in boys aged 11-14 years from the Canadian Health Measures Survey, while the opposite is true for breaks in sedentary time (37). However, these associations were not observed in older or younger boys, or in girls of any age.

The limited evidence available to date suggests that characteristics of sedentary behaviour may be less closely associated with cardiometabolic disease risk in children than has previously been reported in adults. However, it should be noted that the studies that have been published to date have focused primarily on nationally representative samples of children and youth $(37,70)$. It is possible that associations between characteristics of sedentary behaviour and cardiometabolic disease risk may be stronger in populations with a family history of obesity, as this has previously been associated with increased childhood cardiometabolic risk (92-94).

Mechanisms by which sedentary behaviour can lead to poor health outcomes in children and youth

A number of mechanisms have been suggested that could explain the reported associations between sedentary behaviour and cardiometabolic disease risk in the pediatric population. Sedentary behaviours are defined by their low energy expenditure, and it has historically been assumed that they displace physical activity (4). This view is supported by a recent randomized crossover study which observed that exposing children to several hours of prolonged sitting did not result in any changes in physical activity levels in the subsequent 24-hour period (95). This suggests that when children engage in a bout of prolonged sedentary behaviour they do not 
compensate by increasing physical activity levels later on, thereby promoting positive energy balance (95). However, other evidence suggests that the displacement of physical activity plays a relatively small role in mediating the relationship between sedentary time and cardiometabolic disease risk in children and youth $(56,67,88,96,97)$. In a systematic review and meta-analysis examining the relationship between sedentary behaviours and physical activity, Marshall et al. (96) reported that while the two are negatively associated, the magnitude of the relationship is too small to be of clinical significance. As noted above, numerous studies have also observed significant associations between sedentary behaviour (whether self-reported or directly measured) and markers of cardiometabolic disease risk independent of physical activity levels in the pediatric population $(9,10,61,70)$. These findings suggest that a lack of physical activity is not the primary factor linking sedentary behaviour with health indicators in this age group.

In contrast to a lack of physical activity, a number of studies suggest that screen-based sedentary behaviours may lead to increased caloric consumption through a variety of mechanisms $(98,99)$. A recent intervention by Harris et al. (100) observed that exposing children to televised food advertisements increases subsequent ad libitum food intake by $45 \%$. Similar results have also been reported by Halford and colleagues $(101,102)$, who reported that the impact of advertisements on increased food intake is seen across all body weight categories, although it is most pronounced among children with obesity (101). They also noted that the ability to recognize food advertisements was positively associated with food intake, and that children with overweight and obesity were more likely to remember food advertisements after being exposed to them, when compared to their lean peers (101). It is possible that television viewing may also result in increased food intake by inducing "mindless eating" (103). An intervention study by Temple et al. (104) found that children spend more time eating and consume roughly twice as many calories while watching a continuous television program, in comparison to a control condition without entertainment. Passive video game playing has also been shown to increase 
food intake and result in a positive energy balance in the pediatric population. A randomized crossover study by Chaput et al. (105) observed that compared to sitting quietly, 1 hour of passive video game play resulted in an $80 \mathrm{kcal}$ increase in ad libitum food intake in adolescent boys. The collective findings suggest that sedentary screen-based sedentary behaviours (in particular television viewing) are likely to result in increased energy intake and positive energy balance in the pediatric population .

Finally, studies in adults suggest that prolonged sitting may have a rapid and direct impact on metabolic health, independent of changes in body weight or other health behaviours $(15,89)$. For example, intervention studies report that even relatively short bouts of uninterrupted sedentary behaviour result in reduced insulin sensitivity, glucose tolerance, and increased triglyceride levels in adults $(20,89,90,106,107)$. In comparison to a day of sitting that included periodic lightintensity walk breaks, Dunstan et al. reported that a day of uninterrupted sitting resulted in a $30 \%$ increase in insulin resistance in a group of overweight and obese adults (90). Similar results have also been reported in normal weight adults $(106,107)$ and may be due to reductions in lipoprotein lipase and glucose transport protein activity at the level of the skeletal muscle $(13,15)$.

To date only one intervention study has examined the acute effect of prolonged sitting in a pediatric population. Saunders et al. (108) exposed healthy 10-14 year olds to a day of uninterrupted sitting, as well as days with periodic interruptions of light and moderate intensity physical activity. In contrast to previous reports in adults (90), they reported that uninterrupted sitting did not have any impact on the insulin, glucose or lipid response to a standardized meal in this population. Although further intervention studies in children and youth are needed, the available evidence suggests that sitting per se may not have a direct deleterious impact on cardiometabolic health in healthy children and youth. Though it could be that the inherent 
metabolic health of children is such that current analytical methods have limited sensitivity to detect subtle, but adverse, physiological changes.

\section{Opportunities for future research}

Although the relationship between certain sedentary behaviours (e.g. screen time) and cardiometabolic disease risk among children and youth are well-established, the impact of sitting per se is far less clear. As discussed above, independent associations between objectively measured sedentary time and cardiometabolic risk have been reported by some, but not all studies. More research is therefore needed to clarify the relationship between objectively measured sedentary behaviour and health indicators in the pediatric age group. Systematic reviews and meta-analyses focusing specifically on objectively measured sedentary behaviour (as opposed to previous reviews that have focused on all sedentary behaviours) may be especially useful in this regard. Standardization of accelerometry methodology would also allow for much easier comparisons across studies. Future research should also examine whether any personal factors such as sex or body weight influence the reporting of screen time, and why some screenbased sedentary behaviours are associated with health outcomes in certain populations, but not others $(9,10)$.

More research is also needed into the role played by specific characteristics of sedentary behaviour in the pediatric population. Only a small number of studies have investigated the impact of sedentary bout length or breaks in sedentary time in the pediatric population, or the importance of sedentary behaviour during different periods of the day $(37,70)$. A better understanding of the characteristics of sedentary behaviour that are most closely associated with cardiometabolic disease risk is needed in order to develop interventions that are maximally efficacious in reducing cardiometabolic risk among children and youth. It is also possible that 
previously unexamined aspects of accelerometry data, such as total movement variability, may provide additional valuable information on movement patterns in the pediatric age group.

Finally, intervention studies are needed that examine the health and behavioural impact of prolonged sitting in the pediatric population. A recent systematic review concluded that uninterrupted sedentary behaviour results in rapid and deleterious changes in insulin sensitivity, glucose tolerance, and lipid levels in adults (89). However, as noted above, these findings have yet to be replicated in children or youth (108). By extension, it is also unclear whether substituting sedentary behaviour for standing or light-intensity physical activity can lead to improvements in cardiometabolic disease risk among the pediatric population. Further research on the relationship between sedentary behaviours and sleep quality and quantity is also required given the importance of a good night's sleep for overall health (109). Additionally, given the decline of outdoor active play observed over recent decades in children and youth (110), more research is urgently needed to better understand the implications of excessive indoor time and its associated sedentary, technology-centered activities on children's health.

\section{Conclusions}

Available evidence suggests that North American children and youth spend between 40 and 60\% of their waking hours engaging in sedentary behaviours (42-44). Markers of adiposity and cardiometabolic risk are positively associated with sedentary behaviour in general, and with screen-based sedentary behaviours in particular. These relationships appear to be due to the influence of screen-based sedentary behaviours on food intake, and may also be due to a direct metabolic impact of prolonged sitting, although this has received little research attention in the pediatric population. More research is needed to investigate the impact of characteristics of sedentary behaviour (sedentary bout length, breaks in sedentary time, etc), and interventions that examine the health and behavioural impact of sitting per se. Despite limited evidence in children 
and youth, reducing sedentary time in addition to increasing physical activity may have a significant role in the prevention of chronic diseases, including diabetes.

\section{Acknowledgements}

TJS is supported by a Doctoral Research Award from the Canadian Institutes of Health Research, a Doctoral Studentship Award from the Canadian Diabetes Association, and an Excellence Scholarship from the University of Ottawa. JPC holds a Junior Research Chair in Healthy Active Living and Obesity Research. Some of the research reported in this review was supported by a Young Investigator's Award to JPC from the Children's Hospital of Eastern Ontario Research Institute.

\section{Author Disclosures}

The authors report no conflicts of interest.

\section{Author Contributions}

The article was conceived and designed by TJS, JPC and MST. TJS wrote the initial draft of the manuscript, while MST and JPC provided critical edits and additions. All authors approved the final manuscript. 


\section{References}

1. Janssen I, LeBlanc AG. Systematic review of the health benefits of physical activity and fitness in school-aged children and youth. Int J Behav Nutr Phys Act. 2010;7(1):40.

2. Andersen LB, Harro M, Sardinha LB, et al. Physical activity and clustered cardiovascular risk in children: a cross-sectional study (The European Youth Heart Study). The Lancet. 2006;368(9532):299-304.

3. Schmitz KH, Jacobs DR, Hong CP, et al. Association of physical activity with insulin sensitivity in children. Int J Obes. 2002;26(10):1310-1316.

4. Sedentary Behaviour Research Network. Letter to the Editor: Standardized use of the terms "sedentary" and "sedentary behaviours". Appl Physiol Nutr Metab Physiol. 2012 Jun;37(3):540-542.

5. Sardinha LB, Andersen LB, Anderssen SA, et al. Objectively Measured Time Spent Sedentary Is Associated With Insulin Resistance Independent of Overall and Central Body Fat in 9- to 10-Year-Old Portuguese Children. Diabetes Care. 2008;31(3):569-575.

6. Danielsen Y, Júlíusson P, Nordhus I, et al. The relationship between life-style and cardiometabolic risk indicators in children: the importance of screen time. Acta Paediatr. 2011;100(2):253-259.

7. Ekelund U, Brage S, Froberg K, et al. TV Viewing and Physical Activity Are Independently Associated with Metabolic Risk in Children: The European Youth Heart Study. PLOS Med. 2006;3(12):e488.

8. Mark AE, Janssen I. Relationship between screen time and metabolic syndrome in adolescents. J Public Health. 2008;30(2):153-160.

9. Goldfield GS, Saunders TJ, Kenny GP, et al. Screen Viewing and Diabetes Risk Factors in Overweight and Obese Adolescents. Am J Prev Med. 2013;44(4):S364-S370.

10. Goldfield GS, Kenny GP, Hadjiyannakis S, et al. Video game playing is independently associated with blood pressure and lipids in overweight and obese adolescents. PLOS One. 2011;6(11):e26643.

11. Tremblay MS, LeBlanc AG, Janssen I, et al. Canadian Sedentary Behaviour Guidelines for Children and Youth. Appl Physiol Nutr Metab. 2011;36(1):59-64.

12. Cliff DP, Okely AD, Burrows TL, et al. Objectively measured sedentary behavior, physical activity, and plasma lipids in overweight and obese children. Obesity (Silver Spring). 2013;21(2):382-385. 
13. Hamilton MT, Hamilton DG, Zderic TW. Role of low energy expenditure and sitting in obesity, metabolic syndrome, type 2 diabetes, and cardiovascular disease. Diabetes. 2007;56(11):2655-2667.

14. Hamilton MT, Hamilton DG, Zderic TW. Exercise physiology versus inactivity physiology: an essential concept for understanding lipoprotein lipase regulation. Exerc Sport Sci Rev. 2004;32(4):161-166.

15. Tremblay MS, Colley RC, Saunders TJ, et al. Physiological and health implications of a sedentary lifestyle. Appl Physiol Nutr Metab. 2010;35(6):725-740.

16. Hamilton MT, Healy GN, Dunstan DW, et al. Too little exercise and too much sitting: inactivity physiology and the need for new recommendations on sedentary behavior. Curr Cardiovasc Risk Reports. 2008;2(4):292-298.

17. Bergouignan A, Rudwill F, Simon C, et al. Physical inactivity as the culprit of metabolic inflexibility: evidence from bed-rest studies. J Appl Physiol. 2011;111(4):1201-1210.

18. Pate RR, O’Neill JR, Lobelo F. The Evolving Definition of “Sedentary.” Exerc Sport Sci Rev. 2008;36(4):173-178.

19. Manson JE Skerrett PJ, Greenland P. The escalating pandemics of obesity and sedentary lifestyle: A call to action for clinicians. Arch Intern Med. 2004;164(3):249-258.

20. Wilmot EG, Edwardson CL, Achana FA, et al. Sedentary time in adults and the association with diabetes, cardiovascular disease and death: systematic review and metaanalysis. Diabetologia. 2012;55(11):2895-2905.

21. Lynch BM. Sedentary Behavior and Cancer: A Systematic Review of the Literature and Proposed Biological Mechanisms. Cancer Epidemiol Biomarkers Prev. 2010;19(11):2691 -2709 .

22. Lubans DR, Hesketh K, Cliff DP, et al. A systematic review of the validity and reliability of sedentary behaviour measures used with children and adolescents. Obes Rev. 2011;12(10):781-799.

23. Colley RC, Wong SL, Garriguet D, et al. Physical activity, sedentary behaviour and sleep in Canadian children: parent-report versus direct measures and relative associations with health risk. Health Rep. 2012;23(2):45-52.

24. Adamo KB, Prince SA, Tricco AC, et al. A comparison of indirect versus direct measures for assessing physical activity in the pediatric population: a systematic review. Int $J$ Pediatr Obes. 2009;4(1):2-27. 
25. Prince SA, Adamo KB, Hamel ME, et al. A comparison of direct versus self-report measures for assessing physical activity in adults: a systematic review. Int J Behav Nutr Phys Act. 2008;5(1):56.

26. Saunders TJ, Prince SA, Tremblay MS. Clustering of children's activity behaviour: the use of self-report versus direct measures. Int J Behav Nutr Phys Act. 2011;8:48.

27. Biddle SJ, Gorely T, Marshall SJ. Is television viewing a suitable marker of sedentary behavior in young people? Ann Behav Med. 2009;38(2):147-153.

28. Sugiyama T, Healy GN, Dunstan DW, et al. Is television viewing time a marker of a broader pattern of sedentary behavior? Ann Behav Med. 2008;35(2):245-250.

29. Cain KL, Sallis JF, Conway TL, et al. Using accelerometers in youth physical activity studies: a review of methods. $J$ Phys Act Health. 2013;10(3):437-450.

30. Treuth M S, Schmitz KH, Catellier D, et al. Defining Accelerometer Thresholds for Activity Intensities in Adolescent Girls. Med Sci Sports Exerc. 2004;36(7):1259-1266.

31. Evenson KR, Catellier DJ, Gill K, et al. Calibration of two objective measures of physical activity for children. $J$ Sports Sci. 2008;26(14):1557-1565.

32. Ridgers ND, Salmon J, Ridley K, et al. Agreement between activPAL and ActiGraph for assessing children's sedentary time. Int J Behav Nutr Phys Act. 2012;9(1):15.

33. Trost SG, Pate RR, Sallis JF, et al. Age and gender differences in objectively measured physical activity in youth. Med Sci Sports Exerc. 2002;34:350-355.

34. Trost SG, Loprinzi PD, Moore R, et al. Comparison of accelerometer cut points for predicting activity intensity in youth. Med Sci Sports Exerc. 2011;43: 1360-1368.

35. Puyau MR, Adolph AL, Vohra FA, et al.Prediction of activity energy expenditure using accelerometers in children. Med Sci Sports Exerc. 2004;36: 1625-1631.

36. Wong SL, Colley R, Connor-Gorber S, et al. Actical accelerometer sedentary activity thresholds for adults. J Phys Act Health. 2011; 8: 587-591.

37. Colley RC, Garriguet D, Janssen I, et al. The association between accelerometermeasured patterns of sedentary time and health risk in children and youth: a crosssectional study. BMC Public Health. 2013;13(200).

38. Healy GN, Dunstan DW, Salmon J, et al. Breaks in sedentary time: beneficial associations with metabolic risk. Diabetes Care. 2008;31(4):661-666.

39. Healy GN, Matthews CE, Dunstan DW, et al. Sedentary time and cardio-metabolic biomarkers in US adults: NHANES 2003-06. Eur Heart J. 2011;32(5):590-597. 
40. Dowd KP, Harrington DM, Donnelly AE. Criterion and Concurrent Validity of the $\operatorname{activPAL}^{\mathrm{TM}}$ Professional Physical Activity Monitor in Adolescent Females. PLOS One. 2012;7(10):e47633.

41. Aminian S, Hinckson EA. Examining the validity of the ActivPAL monitor in measuring posture and ambulatory movement in children. Int J Behav Nutr Phys Act. 2012;9(1):119.

42. Colley R, Garriguet D, Janssen I, et al. Physical activity of Canadian children and youth: accelerometer results from the 2007 to 2009 Canadian Health Measures Survey. Health Rep. 2011;22(1):15-24.

43. Matthews CE, Chen KY, Freedson PS, et al. Amount of Time Spent in Sedentary Behaviors in the United States, 2003-2004. Am J Epidemiol. 2008;167(7):875-881.

44. Pate RR, Mitchell JA, Byun W, et al. Sedentary behaviour in youth. Br J Sports Med. 2011;45(11):906-913.

45. Trang NH, Hong TK, van der Ploeg HP, et al. Longitudinal Sedentary Behavior Changes in Adolescents in Ho Chi Minh City. Am J Prev Med. 2013;44(3):223-230.

46. Brodersen NH, Steptoe A, Boniface DR, et al. Trends in physical activity and sedentary behaviour in adolescence: ethnic and socioeconomic differences. Br J Sports Med. 2007;41(3):140-144.

47. Kwon S, Burns TL, Levy SM, et al. Breaks in sedentary time during childhood and adolescence: Iowa bone development study. Med Sci Sports Exerc. 2012;44(6):10751080.

48. Schramm W, Lyle J, Parker E. Television in the Lives of Our Children. Stanford University Press; 1961.

49. Rideout V, Foehr U, Roberts D. Generation M2: Media in the Lives of 8- to 18-YearOlds. Kaiser Family Foundation; 2010. [cited 2013 May 14]. Available from: http://kff.org/other/event/generation-m2-media-in-the-lives-of/

50. Leatherdale ST, Ahmed R. Screen-based sedentary behaviours among a nationally representative sample of youth: are Canadian kids couch potatoes? Chronic Dis Inj Can. 2011;31(4):141-146.

51. Sigmundova D, El Ansari W, Sigmund E, et al. Secular trends: a ten-year comparison of the amount and type of physical activity and inactivity of random samples of adolescents in the Czech Republic. BMC Public Health. 2011;11:731.

52. Mak K-K, Day JR. Secular trends of sports participation, sedentary activity and physical self-perceptions in Hong Kong adolescents, 1995-2000. Acta Paediatr.

2010;99(11):1731-1734. 
53. Cui Z, Hardy LL, Dibley MJ, et al. Temporal trends and recent correlates in sedentary behaviours in Chinese children. Int J Behav Nutr Phys Act. 2011;8:93.

54. Øverby NC, Klepp K-I, Bere E. Changes in screen time activity in Norwegian children from 2001 to 2008: two cross sectional studies. BMC Public Health. 2013;13(1):80.

55. Australian Government Department of Health and Ageing. Sedentary behaviour and screen-time. [cited 2013 May 14]. Available from:

http://www.health.gov.au/internet/publications/publishing.nsf/Content/gug-directortoc $\sim$ gug-sedentary

56. Biddle SJH, Gorely T, Marshall SJ, et al. Physical activity and sedentary behaviours in youth: issues and controversies. J R Soc Promot Health. 2004;124(1):29-33.

57. Silva KS, Lopes A da S, Dumith SC, Garcia LMT, Bezerra J, Nahas MV. Changes in television viewing and computers/videogames use among high school students in Southern Brazil between 2001 and 2011. Int J Public Health. In Press.

58. Buliung RN, Mitra R, Faulkner G. Active school transportation in the Greater Toronto Area, Canada: an exploration of trends in space and time (1986-2006). Prev Med. 2009;48(6):507-512.

59. McDonald NC. Active transportation to school: trends among U.S. schoolchildren, 19692001. Am J Prev Med. 2007;32(6):509-516.

60. Van der Ploeg HP, Merom D, Corpuz G, et al. Trends in Australian children traveling to school 1971-2003: burning petrol or carbohydrates? Prev Med. 2008;46(1):60-62.

61. Tremblay M, LeBlanc AG, Kho M, et al. Systematic review of sedentary behaviour and health indicators in school-aged children and youth. Int J Behav Nutr Phys Act. 2011;8(1):98.

62. Hernández B, Gortmaker SL, Colditz GA, et al. Association of obesity with physical activity, television programs and other forms of video viewing among children in Mexico city. Int J Obes Relat Metab Disord. 1999;23(8):845-854.

63. Kaur H, Choi WS, Mayo MS, et al. Duration of television watching is associated with increased body mass index. J Pediatr. 2003;143(4):506-511.

64. Mitchell JA, Pate RR, Beets MW, et al. Time spent in sedentary behavior and changes in childhood BMI: a longitudinal study from ages 9 to 15 years. Int J Obes. 2013;37: 54-60.

65. Robinson TN. Reducing children's television viewing to prevent obesity: A randomized controlled trial. JAMA. 1999;282(16):1561-1567. 
66. Epstein LH, Roemmich JN, Robinson JL, et al. A randomized trial of the effects of reducing television viewing and computer use on body mass index in young children. Arch Pediatr Adolesc. Med. 2008;162(3):239-245.

67. Saunders TJ. Potential Contributors to the Canadian Pediatric Obesity Epidemic. ISRN Pediatr. 2011;1-10.

68. Chaput JP, Saunders TJ, Mathieu ME, et al. Combined associations between moderate to vigorous physical activity and sedentary behavior with cardiometabolic risk factors in children. Appl Physiol Nutr Metab. 2013;38(5):477-483.

69. Henderson M, Gray-Donald K, Mathieu ME, et al. How Are Physical Activity, Fitness, and Sedentary Behavior Associated With Insulin Sensitivity in Children? Diabetes Care. 2012;35(6):1272-1278.

70. Carson V, Janssen I. Volume, patterns, and types of sedentary behavior and cardiometabolic health in children and adolescents: a cross-sectional study. BMC Public Health. 2011;11(1):274.

71. Kriska A, Delahanty L, Edelstein S, et al. Sedentary behavior and physical activity in youth with recent onset of type 2 diabetes. Pediatrics. 2013;131(3):e850-856.

72. Wennberg P, Gustafsson PE, Dunstan DW, et al. Television Viewing and Low LeisureTime Physical Activity in Adolescence Independently Predict the Metabolic Syndrome in Mid-Adulthood. Diabetes Care. 2013; 36(7):2090-2097.

73. Hsu YW, Belcher BR, Ventura EE, et al. Physical activity, sedentary behavior, and the metabolic syndrome in minority youth. Med Sci Sports Exerc. 2011;43: 2307-2313.

74. Chaput JP, Lambert M, Mathieu ME, et al. Physical activity vs. sedentary time: independent associations with adiposity in children. Pediatr Obes. 2012;7:251-258.

75. Martinez-Gomez D, Eisenmann JC, Healy GN, et al. Sedentary Behaviors and Emerging Cardiometabolic Biomarkers in Adolescents. J Pediatr. 2012;160(1):104-110.

76. Basterfield L, Pearce MS, Adamson AJ, et al. Physical Activity, Sedentary Behavior, and Adiposity in English Children. Am J Prev Med. 2012;42: 445-451.

77. Martinez-Gomez D TJ. Associations between sedentary behavior and blood pressure in young children. Arch Pediatr Adolesc Med. 2009;163(8):724-730.

78. Kwon S, Burns TL, Levy SM, et al. Which Contributes More to Childhood AdiposityHigh Levels of Sedentarism or Low Levels of Moderate-through-Vigorous Physical Activity? The Iowa Bone Development Study. J Pediatr. 2013; 162(6):1169-1174. 
79. Atkin AJ, Ekelund U, Møller NC, et al. Sedentary Time in Children: Influence of Accelerometer Processing on Health Relations. Med Sci Sports Exerc. 2012; 45(6):10971104.

80. Mitchell JA, Mattocks C, Ness AR, et al. Sedentary behavior and obesity in a large cohort of children. Obesity (Silver Spring). 2009;17:1596-1602.

81. Steele RM, van Sluijs EMF, Cassidy A, et al. Targeting sedentary time or moderate- and vigorous-intensity activity: independent relations with adiposity in a population-based sample of 10-y-old British children. Am J Clin Nutr. 2009;90: 1185-1192.

82. Ekelund U, Luan J, Sherar LB, et al. Moderate to vigorous physical activity and sedentary time and cardiometabolic risk factors in children and adolescents. JAMA. 2012;307: 704712.

83. Jago R, Fox KR, Page AS, et al. Physical activity and sedentary behaviour typologies of 10-11 year olds. Int J Behav Nutr Phys Act. 2010;7:59.

84. Huang TT-K, Howarth NC, Lin B-H, et al. Energy intake and meal portions: associations with BMI percentile in U.S. children. Obes Res. 2004;12(11):1875-1885.

85. Nettlefold L, McKay HA, Warburton DER, et al. The challenge of low physical activity during the school day: at recess, lunch and in physical education. Br J Sports Med. 2011;45(10):813-819

86. Abbott RA, Straker LM, Mathiassen SE. Patterning of children's sedentary time at and away from school. Obesity (Silver Spring). 2013;21(1):E131-133

87. Saunders TJ, Chaput JP. Is obesity prevention as simple as turning off the television and having a nap? Br J Nutr. 2012;108(5):946-947.

88. Rey-López JP, Vicente-Rodríguez G, Biosca M, et al. Sedentary behaviour and obesity development in children and adolescents. Nutr Metab Cardiovasc Dis. 2008;18(3):242251.

89. Saunders TJ, Larouche R, Colley RC, et al. Acute Sedentary Behaviour and Markers of Cardiometabolic Risk: A Systematic Review of Intervention Studies. J Nutr Metab. 2012;1-12.

90. Dunstan DW, Kingwell BA, Larsen R, et al. Breaking up prolonged sitting reduces postprandial glucose and insulin responses. Diabetes Care. 2012;35(5):976-983.

91. Oliver M, Schluter PJ, Healy GN, et al. Breaks in Sedentary Time - Associations with Body Size in Pacific Mothers and their Children: Findings from the Pacific Islands Families Study. J Phys Act Health. 2012 In Press. 
92. Boney CM, Verma A, Tucker R, et al. Metabolic Syndrome in Childhood: Association With Birth Weight, Maternal Obesity, and Gestational Diabetes Mellitus. Pediatrics. 2005;115(3):e290-e296.

93. Druet C, Dabbas M, Baltakse V, et al. Insulin resistance and the metabolic syndrome in obese French children. Clin Endocrinol. 2006;64(6):672-678.

94. Reilly JJ, Armstrong J, Dorosty AR, et al. Early life risk factors for obesity in childhood: cohort study. BMJ. 2005;330(7504):1357.

95. Saunders TJ, Chaput JP, Goldfield GS, et al. Children and youth do not compensate for an imposed bout of prolonged sitting by reducing subsequent food intake or increasing physical activity: a randomized crossover study. Br J Nutr. In Press.

96. Marshall SJ, Biddle SJH, Gorely T, et al. Relationships between media use, body fatness and physical activity in children and youth: a meta-analysis. Int J Obes Relat Metab Disord. 2004;28(10):1238-1246..

97. Grund A, Krause H, Siewers M, et al. Is TV viewing an index of physical activity and fitness in overweight and normal weight children? Public Health Nutr 2001;4(06):12451251.

98. Thivel D, Aucouturier J, Doucet E, et al. Daily energy balance in children and adolescents. Does energy expenditure predict subsequent energy intake? Appetite. 2013;60(1):58-64.

99. Pearson N, Biddle SJH. Sedentary behavior and dietary intake in children, adolescents, and adults. A systematic review. Am J Prev Med. 2011;41(2):178-188.

100. Harris JL, Bargh JA, Brownell KD. Priming effects of television food advertising on eating behavior. Health Psychol. 2009;28(4):404-413.

101. Halford JCG, Gillespie J, Brown V, et al. Effect of television advertisements for foods on food consumption in children. Appetite. 2004;42(2):221-225.

102. Halford JCG, Boyland EJ, Hughes G, et al. Beyond-brand effect of television (TV) food advertisements/commercials on caloric intake and food choice of 5-7-year-old children. Appetite. 2007;49(1):263-267.

103. Boulos R, Vikre EK, Oppenheimer S, et al. ObesiTV: How television is influencing the obesity epidemic. Physiol Behav. 2012;107(1):146-153.

104. Temple JL, Giacomelli AM, Kent KM, et al. Television watching increases motivated responding for food and energy intake in children. Am J Clin Nutr. 2007;85(2):355-361.

105. Chaput JP, Visby T, Nyby S, et al. Video game playing increases food intake in adolescents: a randomized crossover study. Am J Clin Nutr. 2011;93(6):1196-1203. 
106. Stephens BR, Granados K, Zderic TW, et al. Effects of 1 day of inactivity on insulin action in healthy men and women: interaction with energy intake. Metabolism. 2011;(7):941-949.

107. Duvivier BMFM, Schaper NC, Bremers MA, et al. Minimal Intensity Physical Activity (Standing and Walking) of Longer Duration Improves Insulin Action and Plasma Lipids More than Shorter Periods of Moderate to Vigorous Exercise (Cycling) in Sedentary Subjects When Energy Expenditure Is Comparable. PLOS One. 2013;8(2):e55542.

108. Saunders TJ, Chaput JP, Goldfield GS, et al. Prolonged sitting and markers of cardiometabolic risk in children and youth: a randomized crossover study. Metabolism. In Press.

109. Knutson KL. Sleep duration and cardiometabolic risk: a review of the epidemiologic evidence. Best Pract Res Clin Endocrinol Metab. 2010;24(5):731-743.

110. Active Healthy Kids Canada. Are we driving our kids to unhealthy habits? [cited 2013 May 14]. 2013. Available from: http://activehealthykids.ca/ReportCard/ReportCardOverview.aspx 


\section{Tables}

Table 2.1 Comparison of reports examining the association between objectively measured sedentary time and markers of adiposity and cardiometabolic disease risk among children and youth.

\begin{tabular}{|c|c|c|c|c|c|c|}
\hline Reference & $\begin{array}{l}\text { Setting } \\
\text { (Population) }\end{array}$ & Age & $\mathrm{N}(\mathrm{M} / \mathrm{F})$ & Accelerometer & Sedentary Cut-Point & Key Findings \\
\hline \multicolumn{7}{|c|}{ No significant associations reported } \\
\hline Colley et al. (37) & $\begin{array}{l}\text { Canada } \\
\text { (General Population) }\end{array}$ & $6-19$ years & $1,608(809 / 799)$ & Actical & $<100 \mathrm{CPM}$ & $\begin{array}{l}\text { Sedentary time was not associated with } \\
\text { BMI, waist circumference, HDL-cholesterol } \\
\text { or systolic or diastolic blood pressure } \\
\text { independent of age, wear time and MVPA. }\end{array}$ \\
\hline $\begin{array}{l}\text { Carson and Janssen } \\
\text { (70) }\end{array}$ & $\begin{array}{l}\text { USA } \\
\text { (General Population) }\end{array}$ & $6-19$ years & $2,527(1,284 / 1,243)$ & Actigraph AM-7124 & $<100 \mathrm{CPM}$ & $\begin{array}{l}\text { Sedentary time was not associated with } \\
\text { clustered cardiometabolic risk after } \\
\text { adjustment for age, gender, race, SES, } \\
\text { smoking, total fat, saturated fat, cholesterol, } \\
\text { and sodium, or after additional adjustment } \\
\text { for MVPA. }\end{array}$ \\
\hline $\begin{array}{l}\text { Martinez-Gomez et } \\
\text { al. (75) }\end{array}$ & $\begin{array}{l}\text { Spain } \\
\text { (General Population) }\end{array}$ & $13-17$ years & $183(95 / 88)$ & ActiGraph GT1M & $<100 \mathrm{CPM}$ & $\begin{array}{l}\text { Sedentary time was not associated with } \\
\text { CRP, adiponectin or other adipokines after } \\
\text { adjustment for sex, age, and pubertal status, } \\
\text { or after further adjustment for BMI and } \\
\text { MVPA. }\end{array}$ \\
\hline Kwon et al. (78) & $\begin{array}{l}\text { USA } \\
\text { (General Population) }\end{array}$ & $8-15$ years & $554(277 / 277)$ & Actigraph 7164 & $<100 \mathrm{CPM}$ & $\begin{array}{l}\text { Sedentary time was not associated with fat } \\
\text { mass after adjustment for height and } \\
\text { MVPA. }\end{array}$ \\
\hline
\end{tabular}




\begin{tabular}{|c|c|c|c|c|c|c|}
\hline Chaput et al. (74) & $\begin{array}{l}\text { Canada } \\
\text { (Children with a } \\
\text { family history of } \\
\text { obesity) }\end{array}$ & $8-11$ years & $550(299 / 251)$ & Actigraph LS 7164 & $<100 \mathrm{CPM}$ & $\begin{array}{l}\text { Sedentary time was not associated with } \\
\text { body fat percentage or waist-to-height ratio } \\
\text { with or without adjustment for age, sex, } \\
\text { sleep duration, energy intake, sexual } \\
\text { maturation, parental SES and BMI or } \\
\text { MVPA. }\end{array}$ \\
\hline $\begin{array}{l}\text { Martinez-Gomez et } \\
\text { al. (77) }\end{array}$ & $\begin{array}{l}\text { USA } \\
\text { (General Population) }\end{array}$ & $3-8$ years & $111(57 / 54)$ & Actigraph 7164 & $<100 \mathrm{CPM}$ & $\begin{array}{l}\text { Sedentary time was not associated with } \\
\text { systolic or diastolic blood pressure after } \\
\text { adjustment for age, sex, height or body fat } \\
\text { percentage. }\end{array}$ \\
\hline \multicolumn{7}{|c|}{ Significant associations reported for at least one outcome, not independent of physical activity. } \\
\hline Sardinha et al. (5) & $\begin{array}{l}\text { Portugal } \\
\text { (General Population) }\end{array}$ & $9-10$ years & $308(161 / 147)$ & MTI Actigraph & $<500 \mathrm{CPM}$ & $\begin{array}{l}\text { Sedentary time positively associated with } \\
\text { insulin resistance after adjustment for sex, } \\
\text { sexual maturity, birth weight, measurement } \\
\text { time and both total and central adiposity. }\end{array}$ \\
\hline Cliff et al. (12) & $\begin{array}{l}\text { Australia } \\
\text { (Overweight and } \\
\text { Obese) }\end{array}$ & $5-10$ years & 126 & Actigraph 7164 & $<100 \mathrm{CPM}$ & $\begin{array}{l}\text { Sedentary time was negatively associated } \\
\text { with HDL-cholesterol, but not triglycerides, } \\
\text { total- or LDL-cholesterol after adjustment } \\
\text { for age, sex, adiposity, and diet. Sedentary } \\
\text { time was not associated with any outcome } \\
\text { after additional adjustment for MVPA. }\end{array}$ \\
\hline Kriska et al. (71) & $\begin{array}{l}\text { USA } \\
\text { (Youth with obesity } \\
\text { and type } 2 \text { diabetes) }\end{array}$ & 10-18 years & 551 & ActiGraph AM7164 & $<1 \mathrm{MET}$ & $\begin{array}{l}\text { Obese youths with T2D were sedentary for } \\
56 \text { more minutes/day than obese youth } \\
\text { without T2D. }\end{array}$ \\
\hline
\end{tabular}




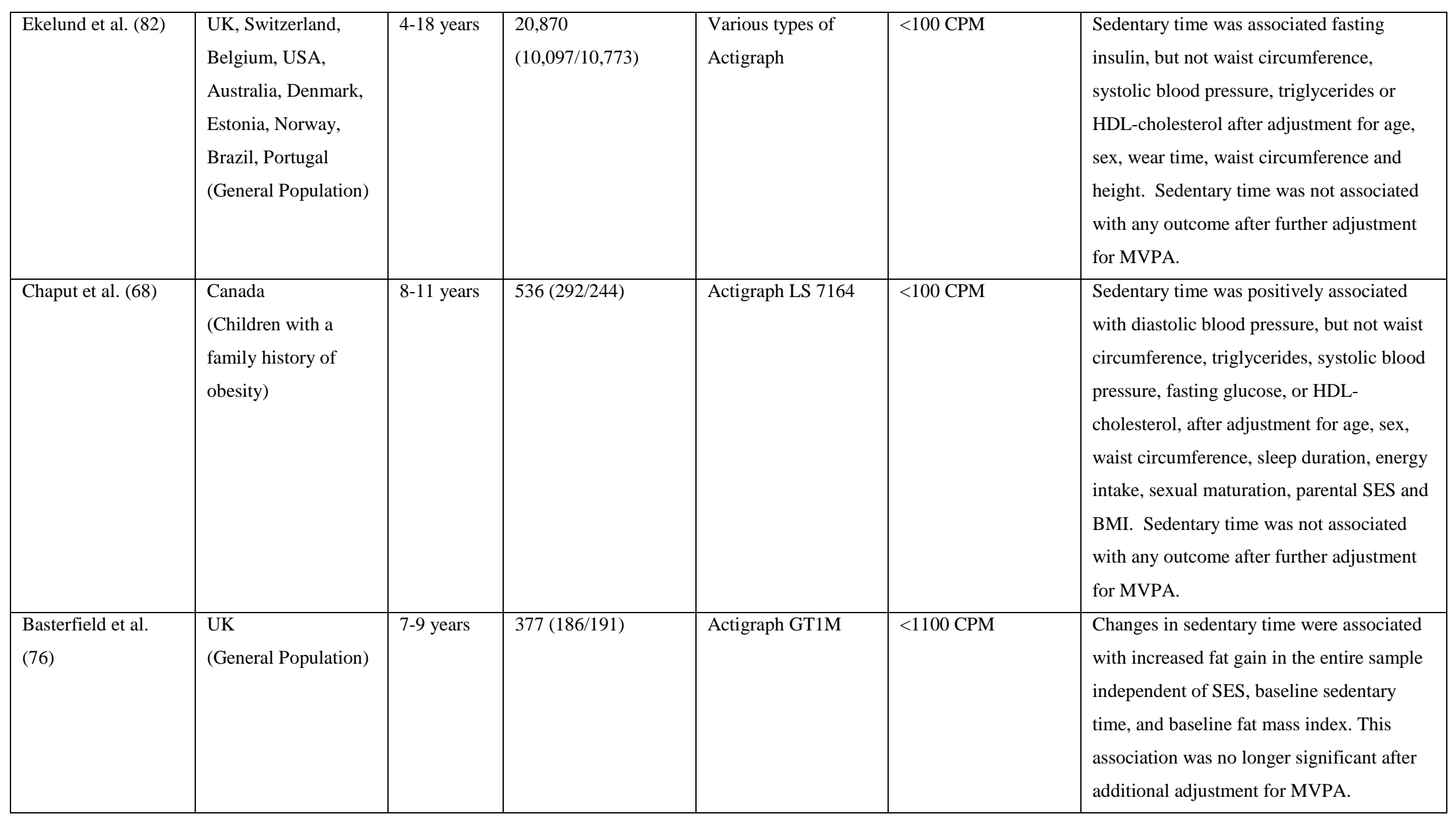




\begin{tabular}{|c|c|c|c|c|c|c|}
\hline Mitchell et al. (80) & $\begin{array}{l}\text { UK } \\
\text { (General Population) }\end{array}$ & 12 years & $5,434(2,950 / 2,844)$ & Actigraph AM7164 & $\leq 199$ CPM & $\begin{array}{l}\text { Sedentary time was significantly associated } \\
\text { with increased risk of obesity independent } \\
\text { gender, SES, pubertal status and early life } \\
\text { sleep and TV habits. These associations } \\
\text { were no longer significant after adjustment } \\
\text { for MVPA. }\end{array}$ \\
\hline Steele et al. (81) & $\begin{array}{l}\text { UK } \\
\text { (General Population) }\end{array}$ & $9-10$ years & $1,862(820 / 1042)$ & Actigraph GT1M & $<100 \mathrm{CPM}$ & $\begin{array}{l}\text { Sedentary time was positively associated } \\
\text { with waist circumference and fat mass } \\
\text { index (but not BMI) in unadjusted analyses. } \\
\text { Sedentary time remained associated with fat } \\
\text { mass index after adjustment for age, sex, } \\
\text { SES, birth weight, sleep duration or } \\
\text { maternal BMI. Sedentary time was not } \\
\text { associated with any outcome after further } \\
\text { adjustment for MVPA. }\end{array}$ \\
\hline
\end{tabular}




\begin{tabular}{|c|c|c|c|c|c|c|}
\hline $\begin{array}{l}\text { Henderson et al. } \\
\text { (69) }\end{array}$ & $\begin{array}{l}\text { Canada } \\
\text { (Children with a } \\
\text { family history of } \\
\text { obesity) }\end{array}$ & $8-11$ years & $424(222 / 202)$ & Actigraph LS 7164 & $<100 \mathrm{CPM}$ & $\begin{array}{l}\text { Sedentary time was positively associated } \\
\text { with insulin resistance after adjustment for } \\
\text { sex, age, pubertal stage, fitness and MVPA, } \\
\text { but not after additional adjustment for } \\
\text { adiposity. }\end{array}$ \\
\hline Mitchell et al. (64) & $\begin{array}{l}\text { USA } \\
\text { (General Population) }\end{array}$ & $9-15$ years & $798(391 / 407)$ & $\begin{array}{l}\text { ActiGraph } 7164 \text { and } \\
\text { GTM1 }\end{array}$ & $<100 \mathrm{CPM}$ & $\begin{array}{l}\text { Sedentary time was associated with weight } \\
\text { gain the 50th 75th and 90th BMI percentiles } \\
\text { independent of gender, race, maternal } \\
\text { education, hours of sleep, healthy eating } \\
\text { scores and MVPA. No significant } \\
\text { associations were observed at lower BMI } \\
\text { percentiles. }\end{array}$ \\
\hline Atkin et al. (79) & $\begin{array}{l}\text { Denmark, Estonia } \\
\text { and Portugal } \\
\text { (General Population) }\end{array}$ & 9,15 years & $2,327(1,059 / 1,268)$ & MTI Actigraph & $\begin{array}{l}<100,<500,<800, \\
\text { and }<1100 \mathrm{CPM}\end{array}$ & $\begin{array}{l}\text { In meta-regression using data from all cut- } \\
\text { points, sedentary time was associated with } \\
\text { increased clustered cardiometabolic disease } \\
\text { risk (but not adiposity) independent of age } \\
\text { group, age, sex, study location, sexual } \\
\text { maturity, day of the week, season, wear } \\
\text { time, adiposity and total physical activity. } \\
\text { The relationship between sedentary time } \\
\text { and clustered risk was stronger at higher } \\
\text { accelerometry thresholds. }\end{array}$ \\
\hline
\end{tabular}

CPM, counts per minute; MVPA, moderate and vigorous physical activity; SES, socioeconomic status; HDL, high density lipoprotein; BMI, body mass index; CRP, C-Reactive Protein. 


\section{Chapter 3 - Study 1: Associations of sedentary behavior, sedentary bouts and breaks in sedentary time with cardiometabolic risk in children with a family history of obesity}

The following article has been accepted for publication in the journal PLOS ONE, and has been formatted according to their requirements. Data from this article has been submitted for presentation at the Canadian Society for Exercise Physiology Annual Scientific Meeting in October, 2013.

Authors: Travis John Saunders ${ }^{1,2}$, Mark Stephen Tremblay ${ }^{1,2}$, Marie-Ève Mathieu ${ }^{3}$, Mélanie Henderson $^{4}$, Jennifer O’Loughlin ${ }^{5}$, Angelo Tremblay ${ }^{6}$ and Jean-Philippe Chaput ${ }^{1,2}$; on behalf of the QUALITY cohort research group

\section{Affiliations:}

${ }^{1}$ Healthy Active Living and Obesity Research Group, Children's Hospital of Eastern Ontario Research Institute, Ottawa, Ontario, Canada. ${ }^{2}$ School of Human Kinetics, Faculty of Health Sciences, University of Ottawa, Ottawa, Ontario, Canada. ${ }^{3}$ Department of Kinesiology, University of Montreal, Montreal, Quebec, Canada. ${ }^{4}$ Department of Pediatrics, CHU Ste-Justine and University of Montreal, Montreal, Quebec, Canada. ${ }^{5}$ Department of Social and Preventive Medicine, Faculty of Medicine, University of Montreal, Montreal, Quebec, Canada. ${ }^{6}$ Department of Kinesiology, Faculty of Medicine, Laval University, Quebec City, Quebec, Canada.

\section{Author Contributions}

Data collection was conducted by members of TEAM PRODIGY, an inter-university research team including Université de Montréal, Concordia University, Université Laval, and McGill University. The analyses included in this article were conceived and designed by TJS, JPC and MST. TJS performed statistical analyses and wrote the initial draft of the manuscript. MST, 
MEM, MH, JO, AT and JPC provided critical edits and additions. All authors approved the final manuscript.

\section{Acknowledgements}

This research was conducted by members of TEAM PRODIGY, an inter-university research team including Université de Montréal, Concordia University, Université Laval, and McGill University. The research team is grateful to all the children and their families who took part in this study as well as the technicians, research assistants and coordinators involved in the QUALITY cohort project. The authors are also grateful to Dr. Katherine Gray-Donald for providing dietary data. Dr. Marie Lambert (July 1952 - February 2012), pediatric geneticist and researcher, initiated the QUALITY cohort. Her leadership and devotion to QUALITY will always be remembered and appreciated. The QUALITY cohort is funded by the Canadian Institutes of Health Research, the Heart and Stroke Foundation of Canada and Fonds de la Recherche en Santé du Québec. The funders had no role in study design, data collection and analysis, decision to publish, or preparation of the manuscript. TJS is supported by Doctoral Research Awards from the Canadian Institutes of Health Research and the Canadian Diabetes Association, as well as an Excellence Scholarship from the University of Ottawa. JOL holds a Canada Research Chair in the Early Determinants of Adult Chronic Disease. AT holds a Canada Research Chair in Environment and Energy Balance. JPC holds a Junior Research Chair in Healthy Active Living and Obesity Research. 


\begin{abstract}
Background: Although reports in adults suggest that breaks in sedentary time are associated with reduced cardiometabolic risk, these findings have yet to be replicated in children.

Purpose: To investigate whether objectively measured sedentary behavior, sedentary bouts or breaks in sedentary time are independently associated with cardiometabolic risk in a cohort of Canadian children aged 8-11 years with a family history of obesity.

Methods: Data from 286 boys and 236 girls living in Quebec, Canada, with at least one biological parent with obesity (QUALITY cohort) were collected from 2005-2008, and analyzed in 2013. Sedentary behavior, light and moderate-to-vigorous physical activity were measured over 7 days using accelerometry. Leisure time computer/video game use and TV viewing over the past 7 days were self-reported. Outcomes included waist circumference, body mass index Zscore, fasting insulin, fasting glucose, triglycerides, HDL-cholesterol, C-reactive protein and a continuous cardiometabolic risk score.
\end{abstract}

Results: After adjustment for confounders, breaks in sedentary time and the number of sedentary bouts lasting 1-4 minutes were associated with reduced cardiometabolic risk score and lower BMI Z-score in both sexes (all $p<0.05$ ). The number of sedentary bouts lasting 5-9 minutes was negatively associated with waist circumference in girls only, while the number of bouts lasting 10-14 minutes was positively associated with fasting glucose in girls, and with BMI Z-score in boys (all $p<0.05$ ). Leisure time computer/video game use was associated with increased cardiometabolic risk score and waist circumference in boys, while TV viewing was associated with increased cardiometabolic risk, waist circumference, and BMI Z-score in girls (all $p<0.05$ ). Conclusions: These results suggest that frequent interruptions in sedentary time are associated with a favourable cardiometabolic risk profile and highlight the deleterious relationship between screen time and cardiometabolic risk among children with a family history of obesity. 


\section{Background}

Sedentary behavior (e.g. sitting or reclining while expending $\leq 1.5$ metabolic equivalents) [1] is independently associated with increased cardiometabolic risk in children and youth [2-10]. Recent systematic reviews have reported that sedentary behavior is associated with reduced cardiorespiratory fitness, increased adiposity and elevated risk of metabolic syndrome in the pediatric age group $[3,4]$. However, while a growing body of evidence suggests that sedentary behavior represents a novel risk factor for chronic disease among children and youth, it is unclear which characteristics and modalities of sedentary behavior are most closely associated with increased health risk in this population $[5,7,8,11]$.

Self-reported screen-based sedentary behaviors (e.g. television viewing, computer use, video game playing, etc.) have been consistently associated with increased markers of cardiometabolic risk in children and youth, independent of physical activity levels [3,5,7-9]. In contrast, studies examining accelerometer-derived measures of sedentary behavior in this age group have often failed to detect a significant association with markers of cardiometabolic risk after adjustment for confounders [5,11-14]. Similarly, while interruptions in objectively measured sedentary time are beneficially associated with markers of cardiometabolic risk in adults $[15,16]$, these findings have yet to be replicated in the pediatric age group $[5,11]$ where activity profiles are highly intermittent [17]. A better understanding of the relationship between characteristics of sedentary behavior and markers of cardiometabolic risk is necessary to inform lifestyle interventions and public health policies aimed at reducing chronic disease risk in children and youth.

The purpose of the present study was to investigate whether objectively measured sedentary time, or characteristics related to the accumulation of sedentary behavior (e.g. breaks in sedentary time or the accumulation of sedentary time in bouts of various lengths) are independently associated with cardiometabolic risk in a cohort of Canadian children aged 8-11 years with a family history 
of obesity. It was hypothesized that a continous cardiometabolic risk score would be positively associated with sedentary behavior, and negatively associated with breaks in sedentary time in this population.

\section{Materials and Methods}

\section{Study population}

The sample consisted of 630 children enrolled in the QUebec Adiposity and Lifestyle InvesTigation in Youth (QUALITY) cohort, which has been described previously [18]. Briefly, participants in the QUALITY cohort are white and aged 8-11 years at study entry, and all participants have at least one biological parent with obesity (i.e. a body mass index (BMI) $\geq 30$ $\mathrm{kg} / \mathrm{m}^{2}$ or abdominal waist circumference $\geq 88 \mathrm{~cm}$ for women or $\geq 102 \mathrm{~cm}$ for men). Children were excluded from the cohort if they were consuming a very low calorie diet ( $\leq 600 \mathrm{kcal} /$ day), had a serious physical or mental health condition that could compromise participation in the study, had diabetes (type 1 or type 2), or were currently taking steroids, $\beta$-blockers, thiazides or other drugs for hypertension.

Roughly 400000 flyers were distributed between 2005 and 2008 to families with children in Grades 2-5, in 1040 primary schools within $75 \mathrm{~km}$ of Montreal, Quebec City and Sherbrooke in Quebec, Canada. Of 3350 families who contacted the study coordinator, 1320 met all inclusion criteria. Reasons for non-participation at baseline among eligible families were: (i) not interested, $81 \%$; (ii) at least one parent did not agree to participate or was unavailable, $11 \%$; (iii) child declined to participate, $4 \%$; (iv) lived too far from a study centre, $2 \%$; (v) insufficient time, $1 \%$; and (vi) other, $1 \%$. 
All data included in the present analysis were collected during baseline examinations between 2005 and 2008. The present cross-sectional analysis was performed in 2013 and includes 522 participants with complete data for all variables of interest.

\section{Ethics Statement}

This project was approved by the institutional ethics review boards at Centre Hospitalier Universitaire Sainte-Justine and Laval University. Written informed parental consent and child assent were obtained for all participants, in accordance with the principles expressed in the Declaration of Helsinki.

\section{Outcome Measures}

All markers of cardiometabolic risk were assessed during a hospital visit. Height was measured to the nearest millimeter using a wall-mounted stadiometer. Weight was assessed to the nearest $0.1 \mathrm{~kg}$ using a spring scale that was calibrated daily. Waist circumference was assessed at the midpoint of the lowest rib and iliac crest at the end of a normal exhalation. Body mass index (BMI) was calculated by dividing body mass $(\mathrm{kg})$ by height in meters squared, and converted to a BMI Z-score based on values published by the Centers for Disease Control and Prevention [19]. All anthropometric measurements were taken in duplicate with participants wearing indoor clothing without shoes or sweaters and measured according to standardized methods by trained research assistants [18].

Metabolic markers were assessed using venous blood samples collected following a 12-hour overnight fast, analyzed in batches at a single site (CHU Sainte-Justine Clinical Biochemistry laboratory) [18]. Plasma insulin was measured with the ultrasensitive Access immunoassay system (Beckman Coulter, Brea, CA, USA). Glucose (oxidase method), HDL-C and triglycerides (enzymatic method) were measured using a Synchron LX, while high sensitivity C-Reactive 
Protein (hs-CRP) (immunoassay method) was measured using a Synchron CX (Beckman Coulter, Brea, CA, USA). Blood pressure was measured on the right arm, with the child in a sitting position and at rest for at least $5 \mathrm{~min}$, using an oscillometric instrument (Dinamap model CR9340, GE Healthcare, Mississauga, ON). Three consecutive measures were obtained with a 1 minute break between each measure. The average value of the 3 measures was used in the present analyses.

\section{Calculation of a Continuous Cardiometabolic Risk Score}

A sex-specific continuous cardiometabolic risk score was calculated for each participant as follows:

$$
\text { Continuous Cardiometabolic Risk Score }=-z H D L+z \text { Insulin }+z \text { Glucose }+
$$

$z$ Triglycerides $+(z B M I+z W C) / 2+(z S B P+z D B P) / 2$

This cardiometabolic risk score was used as a means of estimating an individual's global cardiometabolic risk. In contrast to a dichotomous metabolic syndrome diagnosis, this approach results in a continuous risk score that increases statistical power, and has been used in several recent investigations in the pediatric population $[6,20,21]$.

\section{Physical activity and sedentary behavior}

Objectively measured sedentary behavior and physical activity were assessed using the Actigraph LS 7164 accelerometer (Actigraph, Pensacola, FL, USA) for one week. Participants were instructed to wear the accelerometer on the right hip during all waking hours, except during bathing or aquatic activities such as swimming. Acclerometry data were downloaded as 1-min epochs and were processed using SAS 9.2 (SAS Institute, Cary, NC, USA) and Microsoft Excel (Microsoft, Redmond, WA, USA) according to standardized quality control and data reduction procedures [22]. Non-wear time was defined as at least 60 consecutive minutes of zero counts, with allowance for up to 2 minutes of counts between 0 and 100 [22]. A valid day was defined as 
$\geq 10$ hours of monitor wear time, and only participants with 4 or more valid days (including at least one weekend day) were included in the present analyses. There were no significant differences in any marker of cardiometabolic risk between participants with and without valid accelerometer data (data not shown).

Sedentary behavior was defined as all minutes with an average activity count of less than 100 counts/minute, light physical activity (LPA) as all minutes with an activity count of 100-2296 counts/minute, and moderate-to-vigorous intensity physical activity (MVPA) as any minute with an activity count greater than 2296 counts/minute [23]. A sedentary bout was defined as 1 or more consecutive minutes with less than 100 counts/minute. The number of daily bouts of sedentary time lasting 1-4 minutes, 5-9 minutes, 10-14 minutes, 15-29 minutes, and 30+ minutes were calculated for each participant. Breaks in sedentary time were calculated as any interruption in sedentary time lasting one minute or longer in which the accelerometer counts per minute rose up to or above 100 [15]. Daily television (TV) viewing, and leisure time computer/video game use (surfing the internet, playing video games on a computer or other device, etc.) were assessed using self-report questionnaires. Participants were asked how many hours they spent watching $\mathrm{TV}$ and using the computer for fun on weekdays and weekend days, and a mean score over the 7 days was computed. These questions are similar to those used in the Youth Risk Behavior Survey, and have been shown to be valid and reliable in the pediatric age group [24].

\section{Covariates}

Sexual maturation was assessed by a research nurse and was scored from 1 (pre-pubertal) to 5 (adult) according to Tanner stages $[25,26]$. Ten percent of boys and 35\% of girls had a Tanner stage of 2 or higher, indicating that they had begun puberty. Baseline questionnaires ascertained highest educational level of the parents (high school, pre-university level [Collège 
d'enseignement général et professionnel for Quebec], university) and total annual family income (categorized into 12 groups ranging from $<\$ 10,000$ to $\$ 140,000 \mathrm{CAD}$ or more).

\section{Statistical Analyses}

Sex-by-sedentary behavior interactions were investigated for all outcomes of interest. Significant sex interactions were observed for waist circumference, BMI Z-score, glucose, insulin, and hsCRP, therefore all analyses have been performed in boys and girls separately. Fasting insulin and plasma triglycerides were non-normally distributed and were therefore transformed using a BoxCox transformation prior to their inclusion in statistical analyses.

Independent t-tests were performed to assess differences in behavioral and cardiometabolic risk factors between boys and girls. Simple correlations were used to examine the relationship between self-reported and accelerometer-derived sedentary behavior. Regression analyses were performed to determine the associations between sedentary behavior and both the continuous cardiometabolic risk score and individual markers of cardiometabolic risk. Initial models were unadjusted, while subsequent analyses adjusted for accelerometer wear time, age, light and moderate-to-vigorous physical activity, total sedentary time, BMI Z-score (unless included in the outcome), Tanner stage, parental income and level of education. These covariates were chosen as they were associated with multiple markers of cardiometabolic risk in both sexes (all $\mathrm{p}<0.05)$. Statistical significance was set at a $\mathrm{p}$ value of $<0.05$. All statistical analyses were performed using SAS version 9.2 (SAS Institute, Cary, NC).

\section{Results}

Characteristics of study participants are presented in Table 3.1. In comparison to girls, boys were significantly more physically active and spent more time using computers/playing video games in their leisure time (all $\mathrm{p}<0.01$ ). Boys also had higher concentrations of fasting glucose and HDL- 
Cholesterol and lower diastolic blood pressure, triglycerides and fasting insulin (all $\mathrm{p}<0.01$ ). There were no differences between boys and girls in age, objectively measured sedentary time, LPA, self-reported television viewing, continuous cardiometabolic risk score or any anthropometric measurement (all $\mathrm{p}>0.05$ ). The number of daily sedentary bouts of each length was similar for both sexes. Boys accumulated fewer bouts of sedentary behavior lasting 1-4 minutes $(\mathrm{p}<0.05)$ while there were no differences between sexes for the number of sedentary bouts lasting 5-9 minutes, 15-29 minutes, or 30+ minutes (all p>0.05). Accelerometer-derived sedentary time was positively associated with leisure time computer/video game use in boys only $(\mathrm{r}=0.20, \mathrm{p}=0.008)$, but was not associated with self-reported TV viewing in either sex (all $\mathrm{p}>0.10)$.

\section{Unadjusted Associations}

Associations between characteristics of sedentary behavior and markers of cardiometabolic disease risk are presented in Tables 3.2 and 3.3. In boys, the continuous cardiometabolic risk score was positively associated with total sedentary time, the number of sedentary bouts lasting 10-14 minutes, the number of bouts lasting 15-29 minutes, and both TV viewing and leisure time computer/video game use, while it was negatively associated with the number of sedentary bouts lasting 1-4 minutes (all $\mathrm{p}<0.05$ ). Among girls, the continuous cardiometabolic risk score was positively associated with total sedentary time, sedentary bouts lasting 5-9, 10-14 minutes, and 15-29 minutes, as well as both TV viewing and leisure time computer/video game use (all $\mathrm{p}<$ $0.05)$.

\section{Adjusted Associations}

In the fully adjusted model, breaks in sedentary time were negatively associated with the continuous cardiometabolic risk score (boys: $\beta=-0.057,95 \% \mathrm{CI}=-0.106,-0.008$; girls: $\beta=-$ $0.084,95 \% \mathrm{CI}=-0.143,-0.024)$ and BMI Z-scores (boys: $\beta=-0.026,95 \% \mathrm{CI}=-0.040,-0.012$; 
girls: $\beta=-0.032,95 \% \mathrm{CI}=-0.048,-0.016)$ in both sexes (all $\mathrm{p}<0.05)$. Similar associations were also observed for the number of sedentary bouts lasting 1-4 minutes. The number of sedentary bouts lasting 5-9 minutes was negatively associated with waist circumference in girls only $(\beta=-$ $0.355,95 \% \mathrm{CI}=-0.686,-0.025)(\mathrm{p}<0.05)$. The number of sedentary bouts lasting $10-14$ minutes was positively associated with fasting glucose in girls $(\beta=0.078,95 \% \mathrm{CI}=0.024,0.133)$, and with BMI Z-score in boys $(\beta=0.169,95 \% \mathrm{CI}=0.035,0.302)$. The number of sedentary bouts lasting 15-29 minutes was negatively associated with fasting triglycerides $(\beta=-0.072,95 \% \mathrm{CI}=-$ $0.140,-0.003)$ and hs-CRP $(\beta=-0.279,95 \% \mathrm{CI}=-0.498,-0.060)$ in boys only (all $\mathrm{p}<0.05)$. Finally, leisure time computer/video game use was positively associated with continuous cardiometabolic risk $(\beta=0.485,95 \% \mathrm{CI}=0.084,0.886)$ and waist circumference $(\beta=0.799,95 \%$ $\mathrm{CI}=0.141,1.457)$, and negatively associated with HDL-cholesterol $(\beta=-0.041,95 \% \mathrm{CI}=-0.070,-$ 0.012) in boys only, while TV viewing was positively associated with continuous cardiometabolic risk $(\beta=0.736,95 \% \mathrm{CI}=0.404,1.068)$, waist circumference $(\beta=0.664,95 \% \mathrm{CI}=0.153,1.174)$ and BMI Z-score in girls only $(\beta=0.197,95 \% \mathrm{CI}=0.099,0.294)($ all $\mathrm{p}<0.05)$.

\section{Discussion}

The results of the present study demonstrate that breaks in sedentary time and short bouts of sedentary behavior (e.g. those lasting 1-4 minutes) are associated with reduced cardiometabolic risk and BMI Z-scores in children aged 8-11 independent of total sedentary time and physical activity. These cross-sectional results suggest that children who frequently interrupt their sedentary time may experience lower levels of cardiometabolic risk than those who accumulate sedentary behavior with less frequent interruptions. Markers of cardiometabolic risk were also more closely associated with self-reported leisure time computer/video game use and TV viewing than with objectively measured total sedentary time in this population. 
To our knowledge, this is the first study to report a beneficial association between breaks in sedentary time and global cardiometabolic risk in the pediatric population. Healy and colleagues have previously reported that breaks in sedentary time are independently and beneficially associated with adiposity, glucose metabolism, triglyceride levels and hs-CRP in adults $[15,16]$ although recent studies have generally failed to detect similar associations in children and youth [5,11]. Carson and Janssen [5] did not observe any association between breaks in sedentary time and continuous cardiometabolic risk in a representative sample of American children and youth aged 6-19 years. Examining another representative sample Canadian youth aged 6-19 years, Colley et al [11] found that breaks in sedentary time accumulated after 3pm on weekdays were associated with lower waist circumference in boys aged 11-14 years. However, they reported that breaks in sedentary time were not significantly associated with any other outcome in older or younger boys, or in girls of any age.

The explanation for this discrepancy between the present findings and previous investigations in the pediatric age group is not immediately clear. While the present analysis focused on children with a parental history of obesity, previous investigations into the role of breaks in sedentary behavior among children and youth have focused on representative samples of the Canadian [11] and American [5] pediatric populations. Due to differences in study methodology (e.g. participant age range, accelerometer model, etc) it is not possible to directly compare levels of overweight/obesity, markers of cardiometabolic disease risk or MVPA across the three studies. However, it is possible that associations between breaks in sedentary time and cardiometabolic risk may be stronger in the present population with a family history of obesity, as parental obesity has been associated with increased childhood cardiometabolic risk by some [27-29] but not all studies [30]. This difference in study population may help to explain why the present results are more similar to those reported previously by Healy and colleagues in adults $[15,16]$, rather than other investigations in children and youth $[5,11]$. 
Several mechanisms have been proposed which could explain the beneficial associations between breaks in sedentary time, short bouts of sedentary time, and continuous cardiometabolic risk observed in the present study. Imposed bouts of prolonged sedentary behavior have been shown to acutely reduce insulin sensitivity and increase triglyceride levels in adults [31], effects which are likely due to reductions in lipoprotein lipase and glucose transport protein activity in skeletal muscle $[32,33]$. Similarly, frequent walk breaks have been shown to greatly reduce the acute metabolic impact of prolonged sitting in overweight adults [34]. If the impact of chronic breaks in sedentary time are similar to those observed acutely in adults, this could provide a plausible mechanism linking frequent interruptions in sedentary behavior with lower levels of cardiometabolic disease risk. However, a recent study by Saunders and colleagues failed to detect any acute impact of prolonged sitting, with or without interruptions, on markers of cardiometabolic risk in healthy children and youth [35]. Therefore, given that breaks and short bouts of sedentary behavior were not independently associated with any individual markers of cardiometabolic risk other than BMI Z-score in the present study, it is also possible that excess body weight may simply predispose children toward less frequent interruptions in sedentary time.

The current finding that cardiometabolic risk appears to be more closely associated with selfreported TV viewing and leisure time computer/video game use than with objectively measured sedentary time is consistent with other findings in the pediatric population [5]. As noted recently by Pereira and Power, self-reported sedentary behaviours are poorly understood at present [36]. As a result, the reason for the discrepancy between objective and subjective measures of sedentary behavior in the present study is not clear. Given that self-report measures often differ dramatically from those based on accelerometry [37,38], it is somewhat surprising that it is selfreported sedentary behaviors which are more consistently associated with health risk in the pediatric population. However, it should be noted that self-reported screen time is only able to 
assess a single form of sedentary behaviour, while accelerometry provides a global measure of time spent sitting. As noted elsewhere, the two measures are therefore assessing different constructs $[39,40]$. This point is underscored by the recent findings of Carson and Janssen, who reported a correlation of just 0.08 between self-reported TV viewing and objectively measured sedentary time in a large sample of American children and youth [5].

The present findings suggest that it may be the behaviors children engage in while seated (e.g. increased food intake), rather than the act of sitting per se, that most strongly influences the development of cardiometabolic risk in the pediatric age group [39, 41-44]. For example, it has been reported that exposure to both video games [44] and television commercials [43] result in increased ad libitum food intake in children and youth. In contrast, sitting passively appears to have no impact on subsequent food intake or other forms of behavioural compensation $(39,43-$ 45]. The relationship between screen-based sedentary behaviours and excess food intake may therefore help to explain the associations observed between TV viewing, leisure time computer/video game use, and markers of cardiometabolic disease risk in the present study. More research into the mechanisms linking self-reported and directly measured sedentary behavior with markers of cardiometabolic risk is clearly warranted.

It is interesting to note that the associations between both self-reported and objectively measured aspects of sedentary behaviour appear to be more closely associated with measures of adiposity than with other markers of cardiometabolic disease risk in the present sample. This may be due to the fact that excess adiposity typically precludes the development of cardiometabolic dysfunction in children and youth [46]. For example, it has been reported that just $4 \%$ of obese adolescents have type 2 diabetes, whereas greater than $90 \%$ of youth with diabetes are overweight or obese [46]. Furthermore, it is known that the duration of obesity is strongly related to the risk of cardiometabolic dysfunction [47]. This may help to explain why sedentary behaviours are 
consistently associated with diabetes and cardiovascular disease in adults [48], despite the relatively few significant associations observed for markers of cardiometabolic disease risk in the present study.

The present study includes several strengths and limitations that warrant mention. The present study included objectively measured sedentary time and cardiometabolic risk factors. However, it was also cross-sectional in nature, precluding the determination of causality. Screen-based sedentary behaviours were assessed via self-report, which may have introduced additional error into the current analyses, when compared with more objective measures. Self-report measures have been shown to systematically over-estimate physical activity in children and youth [38], and it is possible that screen-based sedentary behaviours may be similarly over- or underestimated in this population. However, it should be noted that any error or response bias would be likely to bias the associations between screen-based sedentary behaviours and markers of cardiometabolic disease risk towards the null, which underscores the associations observed in the present analyses. It should also be noted that the accelerometer protocol employed by the present study may have resulted in some light physical activities (e.g. standing still) being inadvertently identified as sedentary behavior. Future studies which employ inclinometers may therefore be able to more accurately distinguish between seated and standing activities [49]. These findings are also based on a sample of white youth with a family history of obesity, and therefore may not generalize to all children or to other age groups.

\section{Conclusions}

The results of the present study demonstrate that breaks in sedentary time and short bouts of sedentary behavior are independently and beneficially associated with markers of cardiometabolic risk in children with a family history of obesity. These results also suggest that cardiometabolic risk is more closely associated with measures of self-reported leisure time screen time than with 
objectively measured sedentary time in this population. Future studies should investigate whether minimizing screen time or introducing frequent interruptions in sedentary time prevent the development of cardiometabolic risk among children with a family history of obesity. 


\section{References}

1. Sedentary Behavior Research Network (2012) Letter to the Editor: Standardized use of the terms "sedentary" and "sedentary behaviors". Appl Physiol Nutr Metab 37(3):540-2.

2. Salmon J, Tremblay MS, Marshall SJ, Hume C (2011) Health risks, correlates, and interventions to reduce sedentary behavior in young people. Am J Prev Med 41(2):197206.

3. Tremblay M, LeBlanc A, Kho M, Saunders TJ, Larouche R, et al (2011) Systematic review of sedentary behavior and health indicators in school-aged children and youth. Int J Behav Nutr Phys Act 8(1):98.

4. LeBlanc AG, Spence JC, Carson V, Connor Gorber S, Dillman C, et al. Systematic review of sedentary behavior and health indicators in the early years (aged 0-4 years) (2012) Appl Physiol Nutr Metab 37(4):753-72.

5. Carson V, Janssen I. Volume, patterns, and types of sedentary behavior and cardiometabolic health in children and adolescents: a cross-sectional study (2011) BMC Public Health 11(1):274.

6. Ekelund U, Brage S, Froberg K, Harro M, Anderssen SA, et al (2006) TV Viewing and Physical Activity Are Independently Associated with Metabolic Risk in Children: The European Youth Heart Study. PLOS Med 3(12):e488.

7. Goldfield GS, Kenny GP, Hadjiyannakis S, Phillips P, Alberga A, et al (2011) Video game playing is independently associated with blood pressure and lipids in overweight and obese adolescents. PLOS ONE 6(11):e26643. 
8. Goldfield GS, Saunders TJ, Kenny GP, Hadjiyannakis S, Phillips P, et al (2013) Screen Viewing and Diabetes Risk Factors in Overweight and Obese Adolescents. Am J Prev Med 44(4):S364-S370.

9. Mark AE, Janssen I (2008) Relationship between screen time and metabolic syndrome in adolescents. J Public Health 30(2):153-160.

10. Atkin AJ, Ekelund U, Møller NC, Froberg K, Sardinha LB, et al (2013) Sedentary Time in Children: Influence of Accelerometer Processing on Health Relations. Med Sci Sports Exerc 45(6): 1097-1104.

11. Colley RC, Garriguet D, Janssen I, Wong SL, Saunders TJ , et al (2013) The association between accelerometer-measured patterns of sedentary time and health risk in children and youth: results from the Canadian Health Measures Survey. BMC Public Health 7;13(1):200.

12. Ekelund U, Luan J, Sherar LB, Esliger DW, Griew P, Cooper A (2012) Moderate to vigorous physical activity and sedentary time and cardiometabolic risk factors in children and adolescents. JAMA 307(7):704-12.

13. Chaput JP, Lambert M, Mathieu ME, Tremblay MS, O’ Loughlin J, Tremblay A (2012). Physical activity vs. sedentary time: independent associations with adiposity in children. Pediatr Obes 7(3):251-8.

14. Chaput JP, Saunders TJ, Mathieu ME, Henderson M, Tremblay MS, et al (2013) Combined associations between moderate to vigorous physical activity and sedentary behavior with cardiometabolic risk factors in children. Appl Physiol Nutr Metab 38(5):477-83. 
15. Healy GN, Dunstan DW, Salmon J, Cerin E, Shaw JE, et al (2008) Breaks in sedentary time: beneficial associations with metabolic risk. Diabetes Care 31(4):661-6.

16. Healy GN, Matthews CE, Dunstan DW, Winkler EAH, Owen N (2011) Sedentary time and cardio-metabolic biomarkers in US adults: NHANES 2003-06. Eur Heart J 32(5):590-7.

17. Bailey RC, Olson J, Pepper SL, Porszasz J, Barstow TJ, Cooper DM (1995) The level and tempo of children's physical activities: an observational study. Med Sci Sports Exerc 27(7):1033-41.

18. Lambert M, Hulst AV, O’Loughlin J, Tremblay A, Barnett TA, et al (2012) Cohort Profile: The Quebec Adipose and Lifestyle Investigation in Youth Cohort. Int J Epidemiol 41(6):1533-44.

19. Kuczmarski RJ, Ogden CL, Guo SS, Grummer-Strawn LM, Flegal KM, et al (2002) 2000 CDC Growth Charts for the United States: methods and development. Vital Health Stat (246):1-190.

20. Franks PW, Ekelund U, Brage S, Wong M-Y, Wareham NJ (2004) Does the Association of Habitual Physical Activity With the Metabolic Syndrome Differ by Level of Cardiorespiratory Fitness? Diabetes Care 27(5):1187 -93.

21. Brage S, Wedderkopp N, Ekelund U, Franks PW, Wareham NJ, et al (2004) Features of the metabolic syndrome are associated with objectively measured physical activity and fitness in Danish children. Diabetes Care 27(9):2141-8. 
22. Colley R, Connor Gorber S, Tremblay MS (2010) Quality control and data reduction procedures for accelerometry-derived measures of physical activity. Health Rep 21(1):63-9.

23. Evenson KR, Catellier DJ, Gill K, Ondrak KS, McMurray RG (2008) Calibration of two objective measures of physical activity for children. J Sports Sci 26(14):1557-65.

24. Schmitz KH, Harnack L, Fulton JE, Jacobs DR, Gao S, et al (2004) Reliability And Validity of a Brief Questionnaire to Assess Television Viewing And Computer Use by Middle School Children. J School Health 74(9):370-377.

25. Marshall WA, Tanner JM (1969) Variations in pattern of pubertal changes in girls. Arch Dis Child 44(235):291-303.

26. Marshall WA, Tanner JM (1970) Variations in the pattern of pubertal changes in boys. Arch Dis Child 45(239):13-23.

27. Boney CM, Verma A, Tucker R, Vohr BR (2005) Metabolic Syndrome in Childhood: Association With Birth Weight, Maternal Obesity, and Gestational Diabetes Mellitus. Pediatrics 115(3):e290-e296.

28. Druet C, Dabbas M, Baltakse V, Payen C, Jouret B, et al (2006) Insulin resistance and the metabolic syndrome in obese French children. Clin Endocrinol 64(6):672-8.

29. Reilly JJ, Armstrong J, Dorosty AR, Emmett PM, Ness A, et al (2005) Early life risk factors for obesity in childhood: cohort study. BMJ 330(7504):1357.

30. Ajala O, Hosking J, Metcalf BS, Jeffery AN, Voss LD, Wilkin TJ (2012) The contribution of parental BMI to the metabolic health of their offspring. Pediatr Obes 7(2):143-50. 
31. Saunders TJ, Larouche R, Colley RC, Tremblay MS (2012) Acute Sedentary Behavior and Markers of Cardiometabolic Risk: A Systematic Review of Intervention Studies. J Nutr Metab 2012:1-12.

32. Hamilton MT, Hamilton DG, Zderic TW (2007) Role of Low Energy Expenditure and Sitting in Obesity, Metabolic Syndrome, Type 2 Diabetes, and Cardiovascular Disease. Diabetes 56(11):2655 -2667.

33. Tremblay MS, Colley RC, Saunders TJ, Healy GN, Owen N (2010) Physiological and health implications of a sedentary lifestyle. Appl Physiol Nutr Metab 35(6):725-40.

34. Dunstan DW, Kingwell BA, Larsen R, Healy GN, Cerin E, et al (2012) Breaking up prolonged sitting reduces postprandial glucose and insulin responses. Diabetes Care 35(5):976-83.

35. Saunders TJ, Chaput JP, Goldfield GS, Colley RC, Kenny GP, et al. (2013) Prolonged sitting and markers of cardiometabolic disease risk in children and youth: A randomized crossover study. Metabolism, In press.

36. Pereira SMP, Power C (2013) Sedentary Behaviours in Mid-Adulthood and Subsequent Body Mass Index. PLOS ONE 8(6): e65791.

37. Saunders TJ, Prince SA, Tremblay MS (2011) Clustering of children's activity behavior: the use of self-report versus direct measures. Int J Behav Nutr Phys Act 8:48.

38. Adamo KB, Prince SA, Tricco AC, Connor Gorber S, Tremblay MS (2008) A comparison of direct versus self-report measures for assessing physical activity in the pediatric population: a systematic review. Int J Ped Obesity 4(1):2-27. 
39. Saunders TJ, Chaput JP, Tremblay MS (2013) Sedentary behaviour as an emerging risk factor for cardiometabolic diseases in children and youth. Canadian J Diabetes, In press.

40. Colley RC, Wong SL, Garriguet D, Janssen I, Connor Gorber S, et al. (2012) Physical activity, sedentary behaviour and sleep in Canadian children: parent-report versus direct measures and relative associations with health risk. Health Rep 23(2):45-52.

41. Chaput JP, Klingenberg L, Astrup A, Sjödin AM (2011) Modern sedentary activities promote overconsumption of food in our current obesogenic environment. Obes Rev 12(5):e12-e20.

42. Saunders TJ, Chaput JP (2012) Is obesity prevention as simple as turning off the television and having a nap? Br J Nutr 108(5):946-7.

43. Harris JL, Bargh JA, Brownell KD (2009) Priming effects of television food advertising on eating behavior. Health Psychol 28(4):404-413.

44. Chaput JP, Visby T, Nyby S, Klingenberg L, Gregersen NT et al. (2011) Video game playing increases food intake in adolescents: a randomized crossover study. Am J Clin Nutr 93(6):1196-1203.

45. Saunders TJ, Chaput JP, Goldfield GS, Colley RC, Kenny GP, et al. (2013) Children and youth do not compensate for an imposed bout of prolonged sitting by reducing subsequent food intake or increasing physical activity: a randomized crossover study. $\mathrm{Br}$ J Nutr, In press.

46. Sinha R, Fisch G, Teague B, Tamborlane WV, Banyas B, et al. (2002) Prevalence of impaired glucose tolerance among children and adolescents with marked obesity. NEJM 346(11):802-10. 
47. Modan M, Karasik A, Halkin H, Fuchs Z, Lusky A, et al. (1986) Effect of past and concurrent body mass index on prevalence of glucose intolerance and type 2 (noninsulin-dependent) diabetes and on insulin response. The Israel study of glucose intolerance, obesity and hypertension. Diabetologia 29(2):82-9.

48. Wilmot EG, Edwardson CL, Achana FA, Davies MJ, Gorely T, et al. (2012) Sedentary time in adults and the association with diabetes, cardiovascular disease and death: systematic review and meta-analysis. Diabetologia 55(11):2895-905.

49. Grant PM, Ryan CG, Tigbe WW, Granat MH (2006) The validation of a novel activity monitor in the measurement of posture and motion during everyday activities. Br J Sports Med 40(12):992-997. 


\section{Tables}

Table 3.1 Characteristics of study participants.

\begin{tabular}{|c|c|c|c|}
\hline & Boy $(n=286)$ & Girl $(n=236)$ & Pvalue \\
\hline Age (years) & $9.2(9.1,9.3)$ & $9.1(9.0,9.2)$ & 0.55 \\
\hline Height (cm) & $139.3(138.4,140.2)$ & $138.4(137.3,139.5)$ & 0.20 \\
\hline Weight (kg) & $38.2(36.9,39.5)$ & $38.1(36.7,39.6)$ & 0.94 \\
\hline $\mathrm{BMI}\left(\mathrm{kg} / \mathrm{m}^{2}\right)$ & $19.4(18.9,19.9)$ & $19.6(19.0,20.1)$ & 0.61 \\
\hline Waist Circumference $(\mathrm{cm})$ & $67.6(66.2,69.0)$ & $67.3(65.8,68.8)$ & 0.82 \\
\hline Sedentary Time (min/day) & $363.5(354.9 \quad 372.1)$ & $366.7(358.1375 .4)$ & 0.61 \\
\hline Number of valid days of accelerometry (days) & $6.5(6.4,6.6)$ & $6.5(6.4,6.6)$ & 0.98 \\
\hline Number of hours of accelerometry data (hours/day) & $13.8(13.7,13.9)$ & $13.6(13.5,13.7)$ & 0.02 \\
\hline LPA (min/day) & $403.9(397.1,410.6)$ & $409.5(402.9,416.1)$ & 0.24 \\
\hline MVPA (min/day) & $61.2(57.8,64.6)$ & $41.2(38.8,43.6)$ & $<0.01$ \\
\hline Sedentary Bouts 1-4 Minutes (number/day) & $67(66,68)$ & $70(69,72)$ & $<.01$ \\
\hline Sedentary Bouts 5-9 Minutes (number/day) & $13(12,14)$ & $13(13,14)$ & 0.58 \\
\hline Sedentary Bouts 10-14 Minutes (number/day) & $4(4,5)$ & $4(4,5)$ & 0.88 \\
\hline Sedentary Bouts 15-29 Minutes (number/day) & $3(3,3)$ & $3(3,3)$ & 0.92 \\
\hline Sedentary Bouts 30+ Minutes (number/day) & $2(2,2)$ & $2(2,2)$ & 0.22 \\
\hline TV viewing (hours/day) & $2.0(1.8,2.2)$ & $1.8(1.6,2.0)$ & 0.12 \\
\hline Computer/video game use (hours/day) & $1.1(0.9,1.2)$ & $0.6(0.5,0.7)$ & $<0.01$ \\
\hline Systolic BP (mmHg) & $95(94,96)$ & $94(93,95)$ & 0.23 \\
\hline Diastolic BP (mmHg) & $49(49,50)$ & $50(50,51)$ & 0.01 \\
\hline Insulin (pmol/L) & $30.1(27.9,32.3)$ & $38.2(34.9,41.5)$ & $<0.01$ \\
\hline Glucose (mmol/L) & $5.00(4.96,5.04)$ & $4.90(4.85,4.94)$ & $<0.01$ \\
\hline HDL-Cholesterol (mmol/L) & $1.22(1.19,1.25)$ & $1.16(1.13,1.19)$ & $<0.01$ \\
\hline Triglycerides (mmol/L) & $0.76(0.72,0.80)$ & $0.89(0.84,0.95)$ & $<0.01$ \\
\hline hs-CRP (mg/L) & $1.09(0.82,1.36)$ & $1.20(0.92,1.48)$ & 0.57 \\
\hline Continuous Cardiometabolic Risk Score & $0.03(-0.41,0.48)$ & $0.03(-0.47,0.53)$ & 0.98 \\
\hline
\end{tabular}

Data presented as means (95\% confidence intervals).

$P$ values represent sex differences assessed using an independent Student's t-test.

Clustered cardiometabolic risk score was calculated by summing z-scores for insulin, glucose, triglycerides, inverted HDL-cholesterol, blood pressure, BMI, and waist circumference for each participant.

BMI, body mass index; LPA, light intensity physical activity; MVPA, moderate-to-vigorous physical activity; TV, television; BP, blood pressure; HDL, high density lipoprotein; hs-CRP, high sensitivity C-reactive protein. 
Table 3.2 Associations of sedentary behavior and physical activity with markers of cardiometabolic risk in boys.

\begin{tabular}{|c|c|c|c|c|c|c|c|c|}
\hline & $\begin{array}{c}\text { Continuous } \\
\text { Cardiometabolic } \\
\text { Risk }\end{array}$ & $\mathrm{WC}(\mathrm{cm})$ & BMI (Z-Score) & Insulin $(\mathrm{pmol} / \mathrm{L})$ & $\begin{array}{l}\text { Glucose } \\
(\mathrm{mmol} / \mathrm{L})\end{array}$ & HDL-C (mmol/L) & $\begin{array}{c}\text { Triglycerides } \\
(\mathrm{mmol} / \mathrm{L})\end{array}$ & hs-CRP (mg/L) \\
\hline \multicolumn{9}{|l|}{ Model 1} \\
\hline Sedentary Time (min/day) & $\begin{array}{c}0.010 \\
(0.004,0.016)^{*}\end{array}$ & $\begin{array}{c}0.040 \\
(0.022,0.059)^{*}\end{array}$ & $\begin{array}{c}0.002 \\
(0.001,0.004)^{*}\end{array}$ & $\begin{array}{c}0.001 \\
(0.001,0.002)^{*}\end{array}$ & $\begin{array}{c}0.0001 \\
(-0.0004,0.001)\end{array}$ & $\begin{array}{c}-0.0003 \\
(-0.0007,0.0001)\end{array}$ & $\begin{array}{c}0.001 \\
(-0.0002,0.001)\end{array}$ & $\begin{array}{c}0.004 \\
(0.001,0.006)^{*}\end{array}$ \\
\hline $\begin{array}{c}\text { Breaks in Sedentary Time } \\
\text { (number/day) }\end{array}$ & $\begin{array}{c}-0.020 \\
(-0.059,0.018)\end{array}$ & $\begin{array}{c}-0.093 \\
(-0.215,0.030)\end{array}$ & $\begin{array}{c}-0.006 \\
(-0.017,0.004)\end{array}$ & $\begin{array}{c}-0.004 \\
(-0.010,0.001)\end{array}$ & $\begin{array}{c}-0.002 \\
(-0.005,0.002)\end{array}$ & $\begin{array}{c}-0.001 \\
(-0.004,0.001)\end{array}$ & $\begin{array}{c}-0.004 \\
(-0.008,0.001)\end{array}$ & $\begin{array}{c}0.002 \\
(-0.014,0.019)\end{array}$ \\
\hline $\begin{array}{c}\text { Sedentary Bouts 1-4 } \\
\text { Minutes (number/day) }\end{array}$ & $\begin{array}{c}-0.065 \\
(-0.100,-0.003)^{*}\end{array}$ & $\begin{array}{c}-0.271 \\
(-0 . .82,-0.161)^{*}\end{array}$ & $\begin{array}{c}-0.019 \\
(-0.028,-0.009)^{*}\end{array}$ & $\begin{array}{c}-0.009 \\
(-0.014,-0.005)^{*}\end{array}$ & $\begin{array}{c}-0.002 \\
(-0.005,0.002)\end{array}$ & $\begin{array}{c}0.001 \\
(-0.002,0.003)\end{array}$ & $\begin{array}{c}-0.005 \\
(-0.010,-0.001)^{*}\end{array}$ & $\begin{array}{c}-0.017 \\
(-0.032,-0.002)^{*}\end{array}$ \\
\hline $\begin{array}{c}\text { Sedentary Bouts 5-9 } \\
\text { Minutes (number/day) }\end{array}$ & $\begin{array}{c}0.069 \\
(-0.076,0.214)\end{array}$ & $\begin{array}{c}0.357 \\
(-0.103,0.816)\end{array}$ & $\begin{array}{c}0.010 \\
(-0.028,0.049)\end{array}$ & $\begin{array}{c}0.006 \\
(-0.015,0.026)\end{array}$ & $\begin{array}{c}-0.004 \\
(-0.018,0.009)\end{array}$ & $\begin{array}{c}-0.008 \\
(-0.018,0.002)\end{array}$ & $\begin{array}{c}0.003 \\
(-0.015,0.021)\end{array}$ & $\begin{array}{c}0.077 \\
(0.014,0.139)^{*}\end{array}$ \\
\hline $\begin{array}{l}\text { Sedentary Bouts 10-14 } \\
\text { Minutes (number/day) }\end{array}$ & $\begin{array}{c}0.594 \\
(0.279,0.908)^{*}\end{array}$ & $\begin{array}{c}2.526 \\
(1.547,3.506)^{*}\end{array}$ & $\begin{array}{c}0.158 \\
(0.074,0.241)^{*}\end{array}$ & $\begin{array}{c}0.078 \\
(0.034,0.121)^{*}\end{array}$ & $\begin{array}{c}-0.010 \\
(-0.041,0.020)\end{array}$ & $\begin{array}{c}-0.026 \\
(-0.048,-0.004)^{*}\end{array}$ & $\begin{array}{c}0.038 \\
(-0.001,0.076)\end{array}$ & $\begin{array}{c}0.219 \\
(0.084,0.354)^{*}\end{array}$ \\
\hline $\begin{array}{l}\text { Sedentary Bouts 15-29 } \\
\text { Minutes (number/day) }\end{array}$ & $\begin{array}{c}0.391 \\
(0.059,0.723)^{*}\end{array}$ & $\begin{array}{c}2.071 \\
(1.034,3.107)^{*}\end{array}$ & $\begin{array}{c}0.122 \\
(0.034,0.210)^{*}\end{array}$ & $\begin{array}{c}0.063 \\
(0.017,0.108)^{*}\end{array}$ & $\begin{array}{c}0.006 \\
(-0.025,0.038)\end{array}$ & $\begin{array}{c}-0.004 \\
(-0.026,0.019)\end{array}$ & $\begin{array}{c}0.006 \\
(-0.035,0.047)\end{array}$ & $\begin{array}{c}0.126 \\
(-0.016,0.269)\end{array}$ \\
\hline $\begin{array}{l}\text { Sedentary Bouts 30+ } \\
\text { Minutes (number/day) }\end{array}$ & $\begin{array}{c}0.6620 \\
(-0.015,1.256)\end{array}$ & $\begin{array}{c}2.973 \\
(0.962,4.984)^{*}\end{array}$ & $\begin{array}{c}0.217 \\
(0.048,0.386)^{*}\end{array}$ & $\begin{array}{c}0.109 \\
(0.021,0.197)^{*}\end{array}$ & $\begin{array}{c}-0.006 \\
(-0.065,0.053)\end{array}$ & $\begin{array}{c}-0.012 \\
(-0.056,0.031)\end{array}$ & $\begin{array}{c}0.049 \\
(-0.029,0.127)\end{array}$ & $\begin{array}{c}0.066 \\
(-0.209,0.341)\end{array}$ \\
\hline TV Viewing (hours/day) & $\begin{array}{c}0.465 \\
(0.204,0.726)^{*}\end{array}$ & $\begin{array}{c}0.904 \\
(0.068,1.740)^{*}\end{array}$ & $\begin{array}{c}0.088 \\
(0.017,0.160)^{*}\end{array}$ & $\begin{array}{c}0.041 \\
(0.005,0.078)^{*}\end{array}$ & $\begin{array}{c}0.024 \\
(-0.0001,0.049)\end{array}$ & $\begin{array}{c}-0.015 \\
(-0.033,0.003)\end{array}$ & $\begin{array}{c}0.027 \\
(-0.006,0.060)\end{array}$ & $\begin{array}{c}0.130 \\
(0.017,0.243)^{*}\end{array}$ \\
\hline $\begin{array}{c}\text { Computer/Video Game } \\
\text { Use (hours/day) }\end{array}$ & $\begin{array}{c}0.687 \\
(0.300,1.073)^{*}\end{array}$ & $\begin{array}{c}1.629 \\
(0.394,2.863)^{*}\end{array}$ & $\begin{array}{c}0.058 \\
(-0.049,0.164)\end{array}$ & $\begin{array}{c}0.066 \\
(0.012,0.119)^{*}\end{array}$ & $\begin{array}{c}0.032 \\
(-0.004,0.068)\end{array}$ & $\begin{array}{c}-0.043 \\
(-0.069,-0.017)^{*}\end{array}$ & $\begin{array}{c}0.028 \\
(-0.021,0.076)\end{array}$ & $\begin{array}{c}0.173 \\
(0.007,0.340)^{*}\end{array}$ \\
\hline
\end{tabular}




\begin{tabular}{|c|c|c|c|c|c|c|c|c|}
\hline & $\begin{array}{c}\text { Continuous } \\
\text { Cardiometabolic } \\
\text { Risk }\end{array}$ & WC $(\mathrm{cm})$ & BMI (Z-Score) & $\begin{array}{l}\text { Insulin } \\
(\mathrm{pmol} / \mathrm{L})\end{array}$ & $\begin{array}{l}\text { Glucose } \\
(\mathrm{mmol} / \mathrm{L})\end{array}$ & $\begin{array}{l}\text { HDL-C } \\
(\mathrm{mmol} / \mathrm{L})\end{array}$ & $\begin{array}{c}\text { Triglycerides } \\
(\mathrm{mmol} / \mathrm{L})\end{array}$ & hs-CRP (mg/L) \\
\hline \multicolumn{9}{|l|}{ Model 2} \\
\hline Sedentary Time (min/day) & $\begin{array}{c}0.011 \\
(-0.019,0.041)\end{array}$ & $\begin{array}{c}0.038 \\
(-0.012,0.087)\end{array}$ & $\begin{array}{c}-0.001 \\
(-0.009,0.008)\end{array}$ & $\begin{array}{c}0.001 \\
(-0.003,0.004)\end{array}$ & $\begin{array}{c}0.002 \\
(-0.001,0.005)\end{array}$ & $\begin{array}{c}-0.001 \\
(-0.003,0.001)\end{array}$ & $\begin{array}{c}-0.0004 \\
(-0.004,0.003)\end{array}$ & $\begin{array}{c}0.003 \\
(-0.009,0.015)\end{array}$ \\
\hline $\begin{array}{c}\text { Breaks in Sedentary Time } \\
\text { (number/day) }\end{array}$ & $\begin{array}{c}-0.057 \\
(-0.106,-0.008)^{*}\end{array}$ & $\begin{array}{c}-0.027 \\
(-0.110,0.057)\end{array}$ & $\begin{array}{c}-0.026 \\
(-0.040,-0.012)^{*}\end{array}$ & $\begin{array}{c}-0.002 \\
(-0.008,0.004)\end{array}$ & $\begin{array}{c}0.001 \\
(-0.005,0.006)\end{array}$ & $\begin{array}{c}-0.001 \\
(-0.005,0.002)\end{array}$ & $\begin{array}{c}-0.005 \\
(-0.011,0.001)\end{array}$ & $\begin{array}{c}0.005 \\
(-0.014,0.025)\end{array}$ \\
\hline $\begin{array}{c}\text { Sedentary Bouts 1-4 } \\
\text { Minutes (number/day) }\end{array}$ & $\begin{array}{c}-0.063 \\
(-0.111,-0.015)^{*}\end{array}$ & $\begin{array}{c}-0.052 \\
(-0.133,0.030)\end{array}$ & $\begin{array}{c}-0.028 \\
(-0.041-0.016)^{*}\end{array}$ & $\begin{array}{c}-0.001 \\
(-0.007,0.005)\end{array}$ & $\begin{array}{c}-0.001 \\
(-0.006,0.004)\end{array}$ & $\begin{array}{c}-0.001 \\
(-0.004,0.003)\end{array}$ & $\begin{array}{c}-0.002 \\
(-0.008,0.004)\end{array}$ & $\begin{array}{c}-0.00002 \\
(-0.019,0.019)\end{array}$ \\
\hline $\begin{array}{l}\text { Sedentary Bouts 5-9 } \\
\text { Minutes (number/day) }\end{array}$ & $\begin{array}{c}-0.048 \\
(-0.245,0.148)\end{array}$ & $\begin{array}{c}0.080 \\
(-0.244,0.404)\end{array}$ & $\begin{array}{c}-0.039 \\
(-0.094,0.015)\end{array}$ & $\begin{array}{c}-0.007 \\
(-0.030,0.017)\end{array}$ & $\begin{array}{c}-0.005 \\
(-0.025,0.015)\end{array}$ & $\begin{array}{c}-0.011 \\
(-0.025,0.003)\end{array}$ & $\begin{array}{c}0.005 \\
(-0.019,0.029)\end{array}$ & $\begin{array}{c}0.047 \\
(-0.029,0.123)\end{array}$ \\
\hline $\begin{array}{l}\text { Sedentary Bouts 10-14 } \\
\text { Minutes (number/day) }\end{array}$ & $\begin{array}{c}0.473 \\
(-0.006,0.952)\end{array}$ & $\begin{array}{c}0.334 \\
(-0.468,1.135)\end{array}$ & $\begin{array}{c}0.169 \\
(0.035,0.302)^{*}\end{array}$ & $\begin{array}{c}0.001 \\
(-0.057,0.060)\end{array}$ & $\begin{array}{c}-0.041 \\
(-0.091,0.009)\end{array}$ & $\begin{array}{c}-0.030 \\
(-0.065,0.004)\end{array}$ & $\begin{array}{c}0.018 \\
(-0.041,0.077)\end{array}$ & $\begin{array}{c}-0.073 \\
(-0.262,0.115)\end{array}$ \\
\hline $\begin{array}{l}\text { Sedentary Bouts 15-29 } \\
\text { Minutes (number/day) }\end{array}$ & $\begin{array}{c}-0.165 \\
(-0.735,0.405)\end{array}$ & $\begin{array}{c}-0.721 \\
(-1.653,0.211)\end{array}$ & $\begin{array}{c}0.128 \\
(-0.029,0.285)\end{array}$ & $\begin{array}{c}-0.033 \\
(-0.101,0.035)\end{array}$ & $\begin{array}{c}-0.004 \\
(-0.063,0.055)\end{array}$ & $\begin{array}{c}0.026 \\
(-0.015,0.067)\end{array}$ & $\begin{array}{c}-0.072 \\
(-0.140,-0.003)^{*}\end{array}$ & $\begin{array}{c}-0.279 \\
(-0.498,-0.060)^{*}\end{array}$ \\
\hline $\begin{array}{l}\text { Sedentary Bouts 30+ } \\
\text { Minutes (number/day) }\end{array}$ & $\begin{array}{c}0.321 \\
(-0.411,1.054)\end{array}$ & $\begin{array}{c}0.194 \\
(-1.021,1.409)\end{array}$ & $\begin{array}{c}0.153 \\
(-0.051,0.357)\end{array}$ & $\begin{array}{c}0.038 \\
(-0.050,0.126)\end{array}$ & $\begin{array}{c}0.004 \\
(-0.070,0.077)\end{array}$ & $\begin{array}{c}0.007 \\
(-0.046,0.060)\end{array}$ & $\begin{array}{c}0.017 \\
(-0.072,0.106)\end{array}$ & $\begin{array}{c}-0.171 \\
(-0.447,0.106)\end{array}$ \\
\hline TV Viewing (hours/day) & $\begin{array}{c}0.249 \\
(-0.020,0.519)\end{array}$ & $\begin{array}{c}-0.176 \\
(-0.623,0.272)\end{array}$ & $\begin{array}{c}0.050 \\
(-0.026,0.125)\end{array}$ & $\begin{array}{c}0.003 \\
(-0.029,0.036)\end{array}$ & $\begin{array}{c}0.020 \\
(-0.008,0.048)\end{array}$ & $\begin{array}{c}-0.001 \\
(-0.021,0.018)\end{array}$ & $\begin{array}{c}-0.006 \\
(-0.039,0.027)\end{array}$ & $\begin{array}{c}0.050 \\
(-0.055,0.155)\end{array}$ \\
\hline $\begin{array}{c}\text { Computer/Video Game } \\
\text { Use (hours/day) }\end{array}$ & $\begin{array}{c}0.485 \\
(0.084,0.886)^{*}\end{array}$ & $\begin{array}{c}0.799 \\
(0.141,1.457)^{*}\end{array}$ & $\begin{array}{c}0.016 \\
(-0.097,0.128)\end{array}$ & $\begin{array}{c}0.031 \\
(-0.017,0.079)\end{array}$ & $\begin{array}{c}0.013 \\
(-0.028,0.055)\end{array}$ & $\begin{array}{c}-0.041 \\
(-0.070-0.012)^{*}\end{array}$ & $\begin{array}{c}0.015 \\
(-0.034,0.064)\end{array}$ & $\begin{array}{c}0.145 \\
(-0.011,0.300)\end{array}$ \\
\hline
\end{tabular}

Model 1. Unadjusted analyses.

Model 2. Adjusted for accelerometer wear time, age, light and moderate-to-vigorous physical activity, total sedentary time (except when

exposure), BMI Z-score (except when included in outcome), Tanner stage, parental income and level of education. Data are presented as beta coefficients (95\% confidence intervals). $\mathrm{n}=286$. 
Associations assessed using linear regression analysis. Data are presented as beta coefficients (95\% confidence intervals). $n=286$.

$*=\mathrm{p}<0.05$

Fasting insulin and plasma triglycerides have been transformed using a Box-Cox transformation.

Continous cardiometabolic risk score was calculated by summing z-scores for insulin, glucose, triglycerides, negative HDL-cholesterol, blood pressure, BMI, and waist circumference for each participant.

BMI, body mass index; HDL-C, high density lipoprotein cholesterol; hs-CRP, high sensitivity c-reactive protein; MVPA, moderate-to-vigorous physical activity; TV, television; WC, waist circumference. 
Table 3.3 Associations of sedentary behavior and physical activity with markers of cardiometabolic risk in girls.

\begin{tabular}{|c|c|c|c|c|c|c|c|c|}
\hline & $\begin{array}{c}\text { Continuous } \\
\text { Cardiometabolic } \\
\text { Risk }\end{array}$ & $\mathrm{WC}(\mathrm{cm})$ & BMI (Z-Score) & Insulin (pmol/L) & Glucose $(\mathrm{mmol} / \mathrm{L})$ & HDL-C $(\mathrm{mmol} / \mathrm{L})$ & $\begin{array}{l}\text { Triglycerides } \\
(\mathrm{mmol} / \mathrm{L})\end{array}$ & hs-CRP (mg/L) \\
\hline \multicolumn{9}{|l|}{ Model 1} \\
\hline Sedentary Time (min/day) & $\begin{array}{c}0.010 \\
(0.002,0.017)^{*}\end{array}$ & $\begin{array}{c}0.034 \\
(0.013,0.056)^{*}\end{array}$ & $\begin{array}{c}0.001 \\
(-0.001,0.003)\end{array}$ & $\begin{array}{c}0.020 \\
(0.001,0.003)^{*}\end{array}$ & $\begin{array}{c}0.001 \\
(-0.00004,0.0010)\end{array}$ & $\begin{array}{c}-0.0002 \\
(-0.001,0.0002)\end{array}$ & $\begin{array}{c}0.0001 \\
(-0.001,0.001)\end{array}$ & $\begin{array}{c}-0.001 \\
(-0.004,0.002)\end{array}$ \\
\hline $\begin{array}{l}\text { Breaks in Sedentary Time } \\
\text { (number/day) }\end{array}$ & $\begin{array}{c}0.013 \\
(-0.039,0.065)\end{array}$ & $\begin{array}{c}-0.020 \\
(-0.177,0.137)\end{array}$ & $\begin{array}{c}-0.009 \\
(-0.023,0.005)\end{array}$ & $\begin{array}{c}0.004 \\
(-0.004,0.012)\end{array}$ & $\begin{array}{c}0.004 \\
(-0.001,0.009)\end{array}$ & $\begin{array}{c}0.001 \\
(-0.002,0.004)\end{array}$ & $\begin{array}{c}-0.00004 \\
(-0.006,0.006)\end{array}$ & $\begin{array}{c}-0.009 \\
(-0.030,0.011)\end{array}$ \\
\hline $\begin{array}{c}\text { Sedentary Bouts 1-4 Minutes } \\
\text { (number/day) }\end{array}$ & $\begin{array}{c}-0.016 \\
(-0.064,0.032)\end{array}$ & $\begin{array}{c}-0.124 \\
(-0.266,0.017)\end{array}$ & $\begin{array}{c}-0.009 \\
(-0.022,0.004)\end{array}$ & $\begin{array}{c}-0.004 \\
(-0.011,0.004)\end{array}$ & $\begin{array}{c}-0.00001 \\
(-0.004,0.004)\end{array}$ & $\begin{array}{c}0.001 \\
(-0.002,0.004)\end{array}$ & $\begin{array}{c}0.0004 \\
(-0.005,0.006)\end{array}$ & $\begin{array}{c}-0.005 \\
(-0.024,0.014)\end{array}$ \\
\hline $\begin{array}{l}\text { Sedentary Bouts 5-9 Minutes } \\
\text { (number/day) }\end{array}$ & $\begin{array}{c}0.211 \\
(0.031,0.391)^{*}\end{array}$ & $\begin{array}{c}0.527 \\
(-0.009,1.063)\end{array}$ & $\begin{array}{c}0.024 \\
(-0.026,0.074)\end{array}$ & $\begin{array}{c}0.043 \\
(0.016,0.071)^{*}\end{array}$ & $\begin{array}{c}0.012 \\
(-0.004,0.029)\end{array}$ & $\begin{array}{c}-0.004 \\
(-0.015,0.007)\end{array}$ & $\begin{array}{c}0.001 \\
(-0.021,0.022)\end{array}$ & $\begin{array}{c}0.013 \\
(-0.058,0.085)\end{array}$ \\
\hline $\begin{array}{l}\text { Sedentary Bouts 10-14 Minutes } \\
\text { (number/day) }\end{array}$ & $\begin{array}{c}0.510 \\
(0.120,0.899)^{*}\end{array}$ & $\begin{array}{c}1.025 \\
(-0.141,2.191)\end{array}$ & $\begin{array}{c}-0.016 \\
(-0.124,0.092)\end{array}$ & $\begin{array}{c}0.087 \\
(0.027,0.146)^{*}\end{array}$ & $\begin{array}{c}0.052 \\
(0.017,0.087)^{*}\end{array}$ & $\begin{array}{c}-0.016 \\
(-0.039,0.008)\end{array}$ & $\begin{array}{c}0.012 \\
(-0.034,0.058)\end{array}$ & $\begin{array}{c}-0.057 \\
(-0.211,0.097)\end{array}$ \\
\hline $\begin{array}{l}\text { Sedentary Bouts 15-29 Minutes } \\
\text { (number/day) }\end{array}$ & $\begin{array}{c}0.413 \\
(0.012,0.814)^{*}\end{array}$ & $\begin{array}{c}1.512 \\
(0.338,2.686)^{*}\end{array}$ & $\begin{array}{c}0.017 \\
(-0.093,0.126)\end{array}$ & $\begin{array}{c}0.086 \\
(0.025,0.146)^{*}\end{array}$ & $\begin{array}{c}0.026 \\
(-0.010,0.062)\end{array}$ & $\begin{array}{c}-0.012 \\
(-0.036,0.012)\end{array}$ & $\begin{array}{c}-0.006 \\
(-0.053,0.040)\end{array}$ & $\begin{array}{c}-0.048 \\
(-0.206,0.110)\end{array}$ \\
\hline $\begin{array}{c}\text { Sedentary Bouts 30+ Minutes } \\
\text { (number/day) }\end{array}$ & $\begin{array}{c}0.442 \\
(-0.314,1.198)\end{array}$ & $\begin{array}{c}1.346 \\
(-0.906,3.597)\end{array}$ & $\begin{array}{c}0.033 \\
(-0.174,0.241)\end{array}$ & $\begin{array}{c}0.130 \\
(0.015,0.246)^{*}\end{array}$ & $\begin{array}{c}0.012 \\
(-0.056,0.080)\end{array}$ & $\begin{array}{c}0.014 \\
(-0.032,0.059)\end{array}$ & $\begin{array}{c}0.044 \\
(-0.044,0.133)\end{array}$ & $\begin{array}{c}-0.248 \\
(-0.544,0.047)\end{array}$ \\
\hline TV Viewing (hours/day) & $\begin{array}{c}0.774 \\
(0.475,1.072)^{*}\end{array}$ & $\begin{array}{c}2.458 \\
(1.557,3.359)^{*}\end{array}$ & $\begin{array}{c}0.176 \\
(0.090,0.261)^{*}\end{array}$ & $\begin{array}{c}0.099 \\
(0.053,0.145)^{*}\end{array}$ & $\begin{array}{c}0.023 \\
(-0.005,0.051)\end{array}$ & $\begin{array}{c}-0.030 \\
(-0.048,-0.011)^{*}\end{array}$ & $\begin{array}{c}0.050 \\
(0.014,0.087)^{*}\end{array}$ & $\begin{array}{c}0.196 \\
(0.074,0.319)^{*}\end{array}$ \\
\hline $\begin{array}{c}\text { Computer/Video Game Use } \\
\text { (hours/day) }\end{array}$ & $\begin{array}{c}0.902 \\
(0.314,1.490)^{*}\end{array}$ & $\begin{array}{c}3.215 \\
(1.456,4.974)^{*}\end{array}$ & $\begin{array}{c}0.191 \\
(0.026,0.356)^{*}\end{array}$ & $\begin{array}{c}0.133 \\
(0.044,0.222)^{*}\end{array}$ & $\begin{array}{c}0.010 \\
(-0.043,0.064)\end{array}$ & $\begin{array}{c}-0.037 \\
(-0.073,-0.001)^{*}\end{array}$ & $\begin{array}{c}0.058 \\
(-0.011,0.127)\end{array}$ & $\begin{array}{c}0.203 \\
(-0.033,0.440)\end{array}$ \\
\hline
\end{tabular}




\begin{tabular}{|c|c|c|c|c|c|c|c|c|}
\hline & $\begin{array}{c}\text { Continuous } \\
\text { Cardiometabolic } \\
\text { Risk }\end{array}$ & $\mathrm{WC}(\mathrm{cm})$ & BMI (Z-Score) & $\begin{array}{l}\text { Insulin } \\
(\mathrm{pmol} / \mathrm{L})\end{array}$ & $\begin{array}{l}\text { Glucose } \\
(\mathrm{mmol} / \mathrm{L})\end{array}$ & $\begin{array}{l}\text { HDL-C } \\
(\mathrm{mmol} / \mathrm{L})\end{array}$ & $\begin{array}{l}\text { Triglycerides } \\
(\mathrm{mmol} / \mathrm{L})\end{array}$ & hs-CRP (mg/L) \\
\hline \multicolumn{9}{|l|}{ Model 2} \\
\hline Sedentary Time (min/day) & $\begin{array}{c}0.014 \\
(-0.353,0.957)\end{array}$ & $\begin{array}{c}-0.015 \\
(-0.056,0.027)\end{array}$ & $\begin{array}{c}0.005 \\
(-0.003,0.014)\end{array}$ & $\begin{array}{c}-0.0001 \\
(-0.003,0.003)\end{array}$ & $\begin{array}{c}-0.0001 \\
(-0.003,0.003)\end{array}$ & $\begin{array}{c}-0.0001 \\
(-0.002,0.002)\end{array}$ & $\begin{array}{c}0.001 \\
(-0.003,0.004)\end{array}$ & $\begin{array}{c}-0.006 \\
(-0.016,0.005)\end{array}$ \\
\hline $\begin{array}{c}\text { Breaks in Sedentary Time } \\
\text { (number/day) }\end{array}$ & $\begin{array}{c}-0.084 \\
(-0.143,-0.024)^{*}\end{array}$ & $\begin{array}{c}-0.012 \\
(-0.102,0.079)\end{array}$ & $\begin{array}{c}-0.032 \\
(-0.049,-0.015)^{*}\end{array}$ & $\begin{array}{c}-0.001 \\
(-0.008,0.007)\end{array}$ & $\begin{array}{c}0.002 \\
(-0.004,0.008)\end{array}$ & $\begin{array}{c}0.0001 \\
(-0.003,0.004)\end{array}$ & $\begin{array}{c}-0.002 \\
(-0.010,0.005)\end{array}$ & $\begin{array}{c}0.009 \\
(-0.014,0.031)\end{array}$ \\
\hline $\begin{array}{l}\text { Sedentary Bouts 1-4 Minutes } \\
\text { (number/day) }\end{array}$ & $\begin{array}{c}-0.097 \\
(-0.153,-0.041)^{*}\end{array}$ & $\begin{array}{c}-0.066 \\
(-0.152,0.019)\end{array}$ & $\begin{array}{c}-0.032 \\
(-0.048,-0.016)^{*}\end{array}$ & $\begin{array}{c}-0.004 \\
(-0.010,0.003)\end{array}$ & $\begin{array}{c}-0.001 \\
(-0.006,0.005)\end{array}$ & $\begin{array}{c}-0.0003 \\
(-0.004,0.003)\end{array}$ & $\begin{array}{c}-0.003 \\
(-0.010,0.004)\end{array}$ & $\begin{array}{c}0.008 \\
(-0.013,0.029)\end{array}$ \\
\hline $\begin{array}{l}\text { Sedentary Bouts 5-9 Minutes } \\
\text { (number/day) }\end{array}$ & $\begin{array}{c}0.041 \\
(-0.192,0.274)\end{array}$ & $\begin{array}{c}-0.355 \\
(-0.686,-0.025)^{*}\end{array}$ & $\begin{array}{c}0.020 \\
(-0.047,0.087)\end{array}$ & $\begin{array}{c}0.004 \\
(-0.023,0.031)\end{array}$ & $\begin{array}{c}0.002 \\
(-0.020,0.024)\end{array}$ & $\begin{array}{c}0.004 \\
(-0.010,0.018)\end{array}$ & $\begin{array}{c}-0.009 \\
(-0.036,0.018)\end{array}$ & $\begin{array}{c}0.012 \\
(-0.072,0.096)\end{array}$ \\
\hline $\begin{array}{l}\text { Sedentary Bouts 10-14 Minutes } \\
\text { (number/day) }\end{array}$ & $\begin{array}{c}0.484 \\
(-0.106,1.073)\end{array}$ & $\begin{array}{c}-0.603 \\
(-1.451,0.245)\end{array}$ & $\begin{array}{c}-0.010 \\
(-0.181,0.162)\end{array}$ & $\begin{array}{c}0.017 \\
(-0.053,0.086)\end{array}$ & $\begin{array}{c}0.078 \\
(0.024,0.133)^{*}\end{array}$ & $\begin{array}{c}-0.026 \\
(-0.062,0.009)\end{array}$ & $\begin{array}{c}0.049 \\
(-0.020,0.118)\end{array}$ & $\begin{array}{c}-0.017 \\
(-0.230,0.196)\end{array}$ \\
\hline $\begin{array}{l}\text { Sedentary Bouts 15-29 Minutes } \\
\text { (number/day) }\end{array}$ & $\begin{array}{c}0.512 \\
(-0.209,1.233)\end{array}$ & $\begin{array}{c}-0.073 \\
(-1.107,0.962)\end{array}$ & $\begin{array}{c}0.157 \\
(-0.049,0.363)\end{array}$ & $\begin{array}{c}-0.028 \\
(-0.112,0.056)\end{array}$ & $\begin{array}{c}-0.001 \\
(-0.069,0.067)\end{array}$ & $\begin{array}{c}-0.020 \\
(-0.064,0.023)\end{array}$ & $\begin{array}{c}0.012 \\
(-0.071,0.096)\end{array}$ & $\begin{array}{c}-0.067 \\
(-0.328,0.194)\end{array}$ \\
\hline $\begin{array}{l}\text { Sedentary Bouts } 30+\text { Minutes } \\
\text { (number/day) }\end{array}$ & $\begin{array}{c}-0.260 \\
(-1.112,0.592)\end{array}$ & $\begin{array}{c}0.241 \\
(-0.980,1.463)\end{array}$ & $\begin{array}{c}-0.061 \\
(-0.308,0.185)\end{array}$ & $\begin{array}{c}0.043 \\
(-0.057,0.144)\end{array}$ & $\begin{array}{c}-0.032 \\
(-0.111,0.046)\end{array}$ & $\begin{array}{c}0.043 \\
(-0.008,0.093)\end{array}$ & $\begin{array}{c}0.062 \\
(-0.038,0.162)\end{array}$ & $\begin{array}{c}-0.240 \\
(-0.542,0.062)\end{array}$ \\
\hline TV Viewing (hours/day) & $\begin{array}{c}0.736 \\
(0.404,1.068)^{*}\end{array}$ & $\begin{array}{c}0.664 \\
(0.153,1.174)^{*}\end{array}$ & $\begin{array}{c}0.197 \\
(0.099,0.294)^{*}\end{array}$ & $\begin{array}{c}0.016 \\
(-0.026,0.058)\end{array}$ & $\begin{array}{c}0.005 \\
(-0.028,0.039)\end{array}$ & $\begin{array}{c}-0.009 \\
(-0.0300 .013)\end{array}$ & $\begin{array}{c}0.030 \\
(-0.012,0.072)\end{array}$ & $\begin{array}{c}0.108 \\
(-0.020,0.235)\end{array}$ \\
\hline $\begin{array}{c}\text { Computer/Video Game Use } \\
\text { (hours/day) }\end{array}$ & $\begin{array}{c}0.560 \\
(-0.076,1.197)\end{array}$ & $\begin{array}{c}0.548 \\
(-0.373,1.468)\end{array}$ & $\begin{array}{c}0.141 \\
(-0.043,0.325)\end{array}$ & $\begin{array}{c}0.021 \\
(-0.054,0.096)\end{array}$ & $\begin{array}{c}0.006 \\
(-0.055,0.066)\end{array}$ & $\begin{array}{c}-0.025 \\
(-0.064,0.013)\end{array}$ & $\begin{array}{c}0.020 \\
(-0.055,0.095)\end{array}$ & $\begin{array}{c}0.105 \\
(-0.126,0.335)\end{array}$ \\
\hline
\end{tabular}

Model 1. Unadjusted analyses.

Model 2. Adjusted for accelerometer wear time, age, light and moderate-to-vigorous physical activity, total sedentary time (except when

exposure), BMI Z-score (except when included in outcome), Tanner stage, parental income and level of education. Data are presented as beta coefficients ( $95 \%$ confidence intervals). $n=286$. 
Associations assessed using linear regression analysis.

Associations assessed using linear regression analysis. Data are presented as beta coefficients ( $95 \%$ confidence intervals). $\mathrm{n}=236$.

$*=\mathrm{p}<0.05$

Fasting insulin and plasma triglycerides have been transformed using a Box-Cox transformation.

Continuous cardiometabolic risk score was calculated by summing z-scores for insulin, glucose, triglycerides, negative HDL-cholesterol, blood pressure, BMI, and waist circumference for each participant.

BMI, body mass index; HDL-C, high density lipoprotein cholesterol; hs-CRP, high sensitivity c-reactive protein; MVPA, moderate-to-vigorous physical activity; TV, television; WC, waist circumference. 


\title{
Chapter 4 - Study 2: Movement variability is associated with clustered cardiometabolic disease risk in American youth.
}

The following article was originally submitted to the journal Diabetes Care, and has been formatted according to their requirements. Data from this article has been submitted for presentation at the Canadian Society for Exercise Physiology Annual Scientific Meeting in October, 2013.

\author{
Authors: Travis J Saunders ${ }^{1,2}$, Valerie Carson $^{3}$, Mark S Tremblay ${ }^{1,2}$ \\ Affiliations: \\ ${ }^{1}$ Healthy Active Living and Obesity Research Group, Children's Hospital of Eastern Ontario \\ Research Institute, Ottawa, Ontario, Canada \\ ${ }^{2}$ School of Human Kinetics, University of Ottawa, Ottawa, Ontario, Canada \\ ${ }^{3}$ Faculty of Physical Education and Recreation, University of Alberta, Edmonton, Alberta, \\ Canada
}

\section{Author Contributions}

TJS, VC and MST designed the current analysis. TJS conducted the statistical analyses and wrote the manuscript. All authors helped revise the manuscript. TJS had full access to all the data in the study and takes responsibility for the integrity of the data and the accuracy of the data analysis.

\section{Acknowledgements}

TJS is supported by Doctoral Research Awards from the Canadian Institutes of Health Research and the Canadian Diabetes Association, as well as an Excellence Scholarship from the University of Ottawa. The funding agencies had no role in the design, analysis, or preparation of the present manuscript. The authors report no conflicts of interest. 


\begin{abstract}
Objective: Both the intensity and volume of physical activity are associated with reduced health risk, while the opposite may be true for prolonged bouts of sedentary behavior. This suggests that movement variability, defined as minute-to-minute changes in accelerometer counts per minute, may also be associated with cardiometabolic disease risk. The purpose of this study was to determine whether movement variability was independently associated with markers of cardiometabolic disease risk in a representative sample of American youth aged 12-17 years.

Research Design and Methods: This study included 1460 adolescents from the 2003/04 and 2005/06 National Health and Nutrition Examination Surveys. Physical activity, sedentary behaviour, and movement variability (defined as minute-to-minute changes in accelerometer counts) were measured over 7 days using accelerometry. Outcomes included waist circumference, body mass index Z-score, fasting insulin, fasting glucose, triglycerides, HDL- and LDLcholesterol, systolic and diastolic blood pressure, and a clustered cardiometabolic disease risk score.
\end{abstract}

Results: Participants in the highest tertile of movement variability were characterized by relatively low levels of sedentary time and high levels of moderate and vigorous physical activity and breaks in sedentary time (all $p<0.05$ ). Movement variability was negatively associated with clustered cardiometabolic disease risk and systolic blood pressure in both sexes independent of physical activity, sedentary time and other covariates (all $p<0.05$ ).

Conclusions: These findings provide evidence that movement variability is independently associated with clustered cardiometabolic disease risk in American youth. Therefore, in addition to targeting increases in physical activity and decreases in sedentary time, frequent changes in movement intensity may be important for optimal health among youth. 


\section{Introduction}

Whether accumulated sporadically or in bouts lasting several minutes, moderate and vigorous physical activity (MVPA) is consistently associated with reduced cardiometabolic disease risk in youth (1-3). Further, the benefits of a given volume of physical activity appear to increase with exercise intensity $(2,4-8)$. Recent findings suggest that even short bouts of vigorous intensity physical activity are likely to have a positive impact on adiposity, cardiorespiratory fitness, blood pressure, vascular function, and insulin sensitivity (5-8). These findings suggest that intense bouts of activity, even when brief in duration, have an important impact on fitness and health.

In contrast to MVPA, emerging research suggests that sedentary behavior (activities that involve sitting or reclining while expending $\leq 1.5$ metabolic equivalents(9)) may be independently associated with increased health risk in youth (10-13). Furthermore, research among adults suggests the manner in which sedentary behavior is accumulated may also influence health risk. In particular, prolonged bouts of uninterrupted sedentary behavior appear to have a rapid and deleterious impact on health $(14,15)$, while interruptions in sedentary time are beneficially associated with body weight, abdominal fat, triglycerides and glucose metabolism in adults (1517) and with waist circumference in boys (18).

The above findings suggest that movement variability, defined as minute-to-minute changes in accelerometer counts per minute (CPM), may account for variation in cardiometabolic disease risk beyond that accounted for by MVPA and sedentary time. In comparison to individuals with low levels of movement variability, individuals with high movement variability are likely to accumulate higher amounts of MVPA, and lower amounts of sedentary behavior. For a given volume of sedentary behavior and MVPA, an individual with a high level of movement variability is also likely to have a greater number of breaks in sedentary time, and higher average physical activity intensity (See Figure 4.1). Furthermore, it has recently been proposed that with 
respect to various biological processes, variability per se may also be beneficial to health (19-23).

For example, mechanical ventilators that include random variations in breathing rate and volume result in improved oxygen saturation and organ function, when compared to ventilators that provide constant output $(19,23)$. To date no studies have examined the relationship between movement variability and cardiometabolic disease risk in any population.

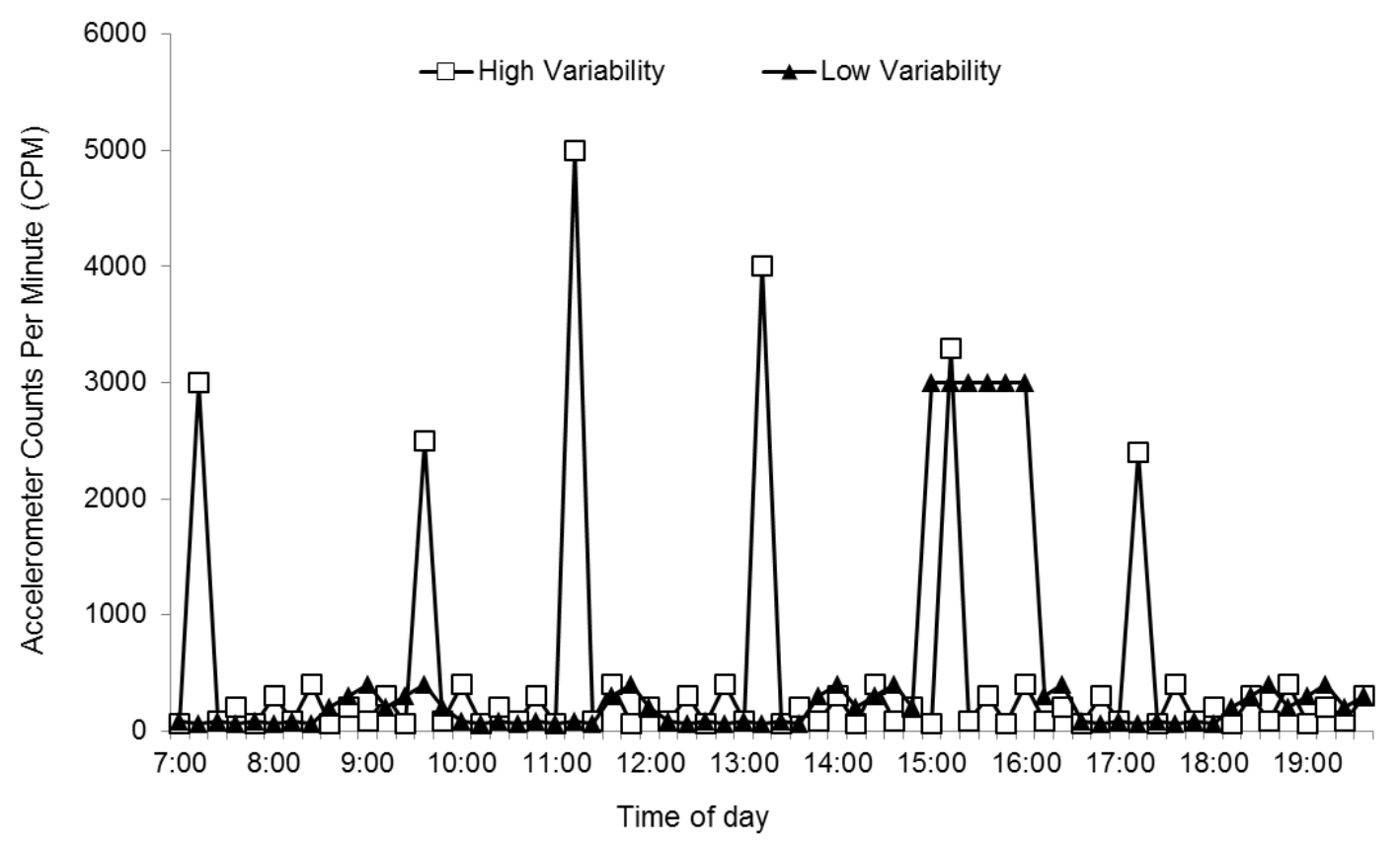

Figure 4.1 Examples of high and low movement variability.

Both individuals have the same amount of total MVPA and sedentary time, although the high variability individual has a cumulative variability of $51080 \mathrm{CPM}$, while the low variability individual has a cumulative variability 10180 CPM.

The purpose of the present study was to investigate the relationship between minute-to-minute movement variability and markers of cardiometabolic disease risk in a representative sample of American youth aged 12-17 years. We hypothesized that movement variability would be associated with improved cardiometabolic disease risk independent of MVPA and sedentary behavior in this population. 


\section{Research Design and Methods}

\section{Participants}

The present study is based on the 2003-2004 and 2005-2006 cycles of the Nutrition Health and Nutrition Examination Survey (NHANES), a nationally representative cross-sectional survey of Americans, which includes both a detailed health interview and a series of direct physical measures. The study was approved by the National Center for Health Statistics, and consent was obtained from all participants and their parents/guardians. A total of 3428 participants aged 1217 took part in the 2003-2004 and 2005-2006 iterations of the NHANES.

Measurement of Physical Activity, Sedentary Behavior, and Movement Variability

Physical activity, sedentary behavior, and movement variability were collected using the Actigraph AM-7124 accelerometer (Actigraph, Ft. Walton Beach, FL), which participants were asked to wear on their right hip for 7 consecutive days except when sleeping or engaging in aquatic activities such as swimming or bathing. Acclerometry data were downloaded as 1-min epochs and were processed using SAS 9.2 (SAS Institute, Cary, NC, USA) according to standardized quality control and data reduction procedures (24). Non-wear time was defined as at least 60 consecutive minutes of zero counts, with allowance for 1 to 2 minutes of counts between 0 and 100 (24). A valid day was defined as $\geq 10$ hours of monitor wear time, and only participants with 4 or more valid days, including at least 1 weekend day, were included in the present analyses. Time spent in moderate ( $\geq 4$ metabolic equivalents) and vigorous intensity physical activity ( $\geq 7$ metabolic equivalents) was determined using age-specific accelerometer cut-points $(25,26)$. Sedentary behavior was defined as $<100$ counts per minute (CPM). A break in sedentary time was defined as any episode lasting 1 minute or longer where the accelerometer count rose to $100 \mathrm{CPM}$ or higher (16). MVPA, VPA, sedentary time, breaks in sedentary time, 
and movement variability were adjusted by wear time by standardizing the variables using the residuals obtained when regressing the variables on wear time (27).

Movement variability was defined as the absolute minute-to-minute variability in accelerometer CPM, for all wear-time on valid days. For example, consecutive minutes with accelerometer counts of 85, 2000 and $150 \mathrm{CPM}$, have a cumulative variability score of $3765 \mathrm{CPM}((|2000-85|)+$ (|150-2000|)). Variability scores were summed for each valid day, and an average daily variability score was calculated for each participant.

\section{Outcome Measures}

All markers of cardiometabolic disease risk, including anthropometric measures, fasting insulin and glucose, triglycerides, HDL- and LDL-cholesterol, and blood pressure were taken by trained personnel at the mobile examination center visit (28). Weight was assessed using a Toledo digital scale (Mettler-Toledo, LLC, Columbus, $\mathrm{OH}$ ) while participants wore a paper gown. Height was assessed using a fixed stadiometer. BMI was calculated by dividing body mass $(\mathrm{kg})$ by height in meters squared, and converted to a BMI Z-score based on values published by the CDC (29). Waist circumference was made at the level of the iliac crest following a normal expiration.

HDL-cholesterol was measured using the direct HDL immunoassay method, while total cholesterol and triglycerides were measured enzymatically. LDL-cholesterol was calculated from measured values of total cholesterol, triglycerides, and HDL-cholesterol according to the Friedewald equation (30). Glucose and insulin measurements differed slightly between 2003 2004 and 2005-2006; glucose was assessed via the hexokinase method using the Roche Cobas Mira (F. Hoffmann-La Roche Ltd, Basel, Switzerland) in 2003-2004, and with the Roche/Hitachi 911 in 2005-2006. Insulin was analyzed via immunoenzymometric assay in 2003-2004 and via ELISAin 2005-2006 (31). A validated regression equation was used to convert values from 
2005-2006 to those which were directly comparable to values from 2003-2004 (31). Blood pressure was measured manually four consecutive times on the right arm while seated, and the average blood pressure was calculated after excluding the first reading, except when only one reading was taken ( $\mathrm{n}=86$ for systolic blood pressure and $\mathrm{n}=113$ for diastolic blood pressure).

\section{Calculation of Clustered Cardiometabolic Disease Risk Score}

An age and sex-specific clustered cardiometabolic disease risk score was calculated for each participant as follows:

Clustered Cardiometabolic Disease Risk Score $=-z H D L+z$ Insulin $+z$ Glucose +

$z$ Triglycerides $+(z B M I+z W C) / 2+(z S B P+z D B P) / 2(32)$

This clustered risk score was used as a means of estimating an individual's global cardiometabolic disease risk, and is based broadly on the metabolic syndrome (32).

\section{Covariates}

Age, sex, self-ascribed ethnicity (non-Hispanic white, non-Hispanic black, Hispanic, other), poverty-to-income ratio (a measure of socioeconomic status (SES), calculated as the ratio between family income and poverty threshold), smoking status, and energy intake were included as covariates. Ethnicity was self-reported by participants, and was included because previous research has observed significant interactions between sedentary behavior and ethnicity in the past (17). Smoking was assessed by asking participants "Have you ever tried cigarette smoking, even 1 or 2 puffs?", and participants were dichotomized to categories of "yes" or "no". Energy intake was assessed by 24-hour recall.

\section{Statistical Analyses}

Insulin, glucose, triglycerides, waist circumference, MVPA, vigorous physical activity (VPA), and breaks in sedentary time were non-normally distributed, and therefore log transformed prior 
to inclusion as the outcome in any statistical analysis. Sex-by-variability and ethnicity-byvariability interactions were investigated for all outcomes of interest. We observed significant sex interactions but not ethnicity interactions for clustered risk, HDL-Cholesterol and triglycerides; therefore, all analyses have been performed in boys and girls separately. T-tests were used to assess differences in movement variability between boys and girls. Linear regression analyses were used to examine the association between movement variability and markers of cardiometabolic disease risk after adjustment for age, ethnicity, SES, energy intake, smoking, and waist circumference (when not an outcome) (Model 1), and after additional adjustment for sedentary behavior and MVPA (Model 2). All regression results are presented as the change in the outcome per 10000 CPM increase in movement variability.

Participants were also divided into sex-specific tertiles of movement variability. Initial ANCOVAs were used to examine whether MVPA, VPA, sedentary behavior or breaks in sedentary time differed by tertile of movement variability, with adjustment for age, ethnicity, SES, energy intake and smoking status. Subsequent ANCOVAs were performed to assess whether the clustered cardiometabolic disease risk score varied according to tertiles of movement variability, after adjustment for age, ethnicity, SES, energy intake, smoking, sedentary behavior and MVPA. A Bonferroni correction was used to adjust for multiple comparisons in post hoc tests following ANCOVAs to assess differences between individual tertiles.

Data are presented as means and 95\% confidence intervals (CI) unless otherwise noted. Statistical significance was set at a $p$ value of $<0.05$. All statistical analyses were performed using SAS version 9.2 (SAS Institute, Cary, NC) and accounted for the complex design and sample weights of NHANES. Due to missing data and significant differences in age and ethnicity between included and excluded participants, sample weights were re-weighted for non-response to achieve a representative sample. 


\section{Results}

Subject characteristics are presented in Table 4.1. Full accelerometry and non-fasting blood sample data were available for 1460 participants ( 707 girls, 753 boys), however only 656 participants (314 girls, 342 boys) had full data for fasting blood samples (insulin, glucose, triglycerides, and LDL-Cholesterol). Thus analyses involving these outcomes and the clustered risk score have a sample of 656 participants, while analyses involving non-fasting outcomes (body mass index (BMI), waist circumference, HDL-Cholesterol, and systolic and diastolic blood pressure) include the full sample of 1460 .

Approximately $52 \%$ of the sample was male, with an average age of 14.9 years. Participants spent $57 \%$ of their time engaging in sedentary behavior, and 3\% engaging in MVPA. On average, participants accumulated 276976 CPM of movement variability during each valid day, with boys accumulating significantly more than girls (Girls: 245747 (238025, 253469); Boys: 306911 (296630, 317193), $p<0.0001)$.

Physical activity and sedentary behavior across tertiles of movement variability are presented in Table 4.2. In both sexes MVPA, VPA and breaks in sedentary time significantly increased across tertiles of movement variability, while sedentary time significantly decreased (all $p<0.0001$ ).

The associations between movement variability and markers of cardiometabolic disease risk are presented in Table 4.3. In both sexes, movement variability was associated with reduced clustered risk and systolic blood pressure independent of MVPA, sedentary time, and other covariates (all $p<0.05)$. Movement variability was also independently associated with reduced diastolic blood pressure in girls, reduced fasting glucose and insulin in boys, and increased BMI in boys (all $p<0.05)$. After back-transforming glucose and insulin from the log scale in boys, an 
additional $10000 \mathrm{CPM}$ of movement variability was associated with a $0.1(0.0,0.2) \%$ lower fasting glucose level, and with a $1.9(0.8,3.0) \%$ lower fasting insulin.

The association between tertiles of movement variability and clustered risk is shown in Figure 4.2. There was a significant trend for reduced clustered risk with increasing tertiles of movement variability independent of MVPA, sedentary time, and other covariates (all $p<0.05$ ) in both sexes. Clustered risk was significantly reduced in the highest tertile of movement variability compared to the lowest tertile in both boys and girls, while risk was also reduced in the middle tertile for boys only (all $p<0.05)$.

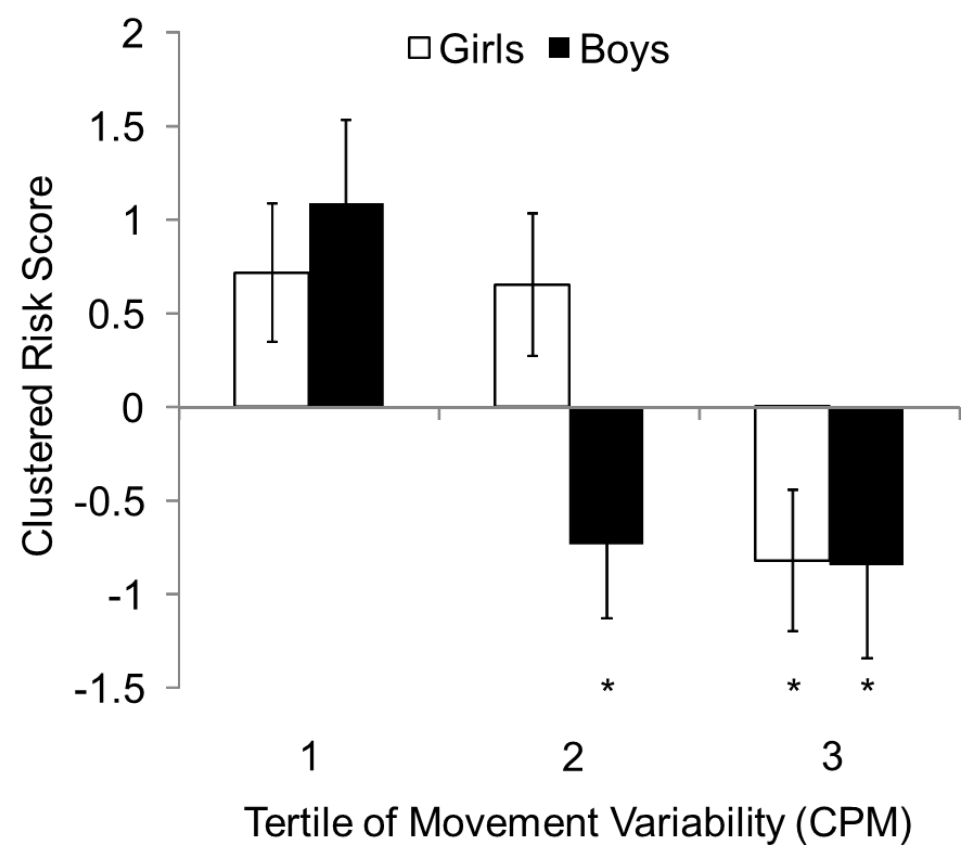

Figure 4.2 Clustered cardiometabolic disease risk across tertiles of movement variability.

Associations assessed using ANCOVAs with adjustment for age, ethnicity, SES, energy intake and smoking status. Data are presented as mean \pm standard error.

*=significantly different from Tertile 1 of same sex, $p<0.05$. 


\section{Conclusions}

The results of the present study support our hypothesis, and demonstrate that total movement variability is beneficially associated with clustered cardiometabolic disease risk in youth independent of covariates including energy intake, MVPA, and sedentary behavior. These findings also illustrate that individuals with high levels of movement variability are characterized by relatively low levels of sedentary behavior, and high amounts of MVPA, VPA, and breaks in sedentary time. These findings suggest that youth with high levels of variability in their movement patterns are likely to experience lower clustered risk than participants with lower levels of movement variability.

This is the first study to investigate the relationship between movement variability and cardiometabolic disease risk in any population, and therefore makes a novel contribution to the literature. However, the clinical and public health significance of these findings is not yet clear. These results suggest that a $100000 \mathrm{CPM}$ increase in daily movement variability could reduce clustered metabolic risk by approximately 1.5 units in boys, and by 1 unit in girls. To put this in perspective, this could be achieved by just 50 transitions from sedentary behavior to light physical activity, or an equivalent number of transitions from light to moderate activity. This increase in variability might be most easily achieved by activities which include alternations of high and low intensity movement, such as soccer or basketball $(33,34)$. Guagliano and colleagues (34) report that during basketball, soccer and netball games, adolescent girls spend roughly $40 \%$ of their time being sedentary, $30 \%$ engaging in light activity, and $15 \%$ in both MPA and VPA. A similar range of movement behaviors have been reported during soccer games involving adolescent boys (33). It is conceivable that household chores such as gardening or sweeping could also contribute to increased variability (35). 
The present findings suggest that for a given energy expenditure, interventions that produce greater increases in total movement variability may bring about greater health benefits in youth. Future research should investigate the health impact of variability per se, and examine whether different methods of increasing movement variability (e.g. frequent breaks in sedentary time vs increased time spent in variable MVPA) bring about comparable improvements in cardiometabolic health. If interventions targeting movement variability are shown to be efficacious in improving cardiometabolic health in at-risk youth, this could lead to interventions which are more feasible and attractive to non-exercisers than traditional programs focusing on structured bouts of MVPA.

There are a number of mechanisms that could explain the results observed in the present study. Variability is a key component of all forms of interval training - by repeated exposure to brief but intense stressors, an individual is exposed to a greater total load than would be possible if the intensity were consistent over time (5). Similar effects are likely to contribute to the beneficial associations observed in the present study. Movement variability was associated with reduced sedentary time, increased breaks in sedentary time, and increased volume of both MVPA and VPA - all factors that have been individually linked with improved health. This suggests that individuals with high levels of movement variability may benefit from the same mechanisms associated with these other movement behaviors, including increased lipoprotein lipase and glucose transport protein activity $(36,37)$, increased mitochondrial capacity (5) and vascular function $(5,37)$. Future research is needed to investigate the mechanisms underlying the relationships observed in the present study, and to determine whether there are benefits related to movement variability per se, or whether it is simply a proxy measure for other important movement behaviors. 
It has recently been argued that with respect to various forms of biological stimuli, variability per se may be beneficial to health (19-23). For example, it has been shown that randomly varying the rate and volume of breaths provided by a mechanical ventilator results in enhanced gas exchange and reduced stress in the lung, heart and brain when compared to ventilators that provide breaths at a constant rate and volume $(19,23)$. Further, it has been noted that disease states such as Parkinson's disease (gait patterns) and congestive heart failure (heart rate variability) are characterized by dramatic reductions in variability (21). Finally, some have suggested that variations in energy intake via intermittent fasting may lead to improvements in markers of cardiometabolic disease risk in adults $(20,22)$. Interestingly, children living in traditional lifestyles, such as Old Order Amish and Mennonite communities, appear to have greater variability in their movement profiles than children living a contemporary lifestyle (38). Given the current findings and those cited above, it is plausible that total movement variability may have a direct influence on markers of cardiometabolic disease risk in youth.

The present study has several important strengths and limitations that warrant mention. Strengths include the objective measurement of key movement variables and markers of cardiometabolic disease risk. Our study also included a large and representative sample of American youth aged 12-17. Limitations include the cross-sectional design, which precludes determinations of causality. Further, several important confounders included in the present study (SES, smoking status, and energy intake) were self-reported, which may have resulted in residual confounding $(39,40)$.

In conclusion, the present study provides evidence that movement variability is associated with improved clustered cardiometabolic disease risk as well as physical activity and sedentary behavior profiles in American youth. These results suggest that independent of MVPA and total sedentary time, increases in movement variability may result in improved health in this age 
group. Further research is needed to investigate the mechanisms underlying these relationships, and to determine whether increased movement variability per se is sufficient to improve health in this population. 


\section{References}

1. Holman RM, Carson V, Janssen I. Does the Fractionalization of Daily Physical Activity (Sporadic vs. Bouts) Impact Cardiometabolic Risk Factors in Children and Youth? PLoS One 2011;6(10):e25733.

2. Janssen I, LeBlanc AG. Systematic review of the health benefits of physical activity and fitness in school-aged children and youth. Int J Behav Nutr Phys Act 2010;7(1):40.

3. Jiménez-Pavón D, Kelly J, Reilly JJ. Associations between objectively measured habitual physical activity and adiposity in children and adolescents: Systematic review. Int J Pediatr Obes 2010;5(1):3-18.

4. Warburton DER, Nicol CW, Bredin SSD. Health benefits of physical activity: the evidence. CMAJ 2006;174(6):801-809.

5. Gibala MJ, Little JP, MacDonald MJ, Hawley JA.Physiological adaptations to lowvolume, high-intensity interval training in health and disease. J Physiol 2012;590(5):1077-1084.

6. Tjønna AE, Stølen TO, Bye A, Volden M, Slørdahl SA, Odegård R, et al. Aerobic interval training reduces cardiovascular risk factors more than a multitreatment approach in overweight adolescents. Clin Sci(Lond)2009;116(4):317-326.

7. Breil FA, Weber SN, Koller S, Hoppeler H, Vogt M. Block training periodization in alpine skiing: effects of 11-day HIT on VO2max and performance. Eur J Appl Physiol 2010;109(6):1077-1086.

8. Hulzebos HJ, Snieder H, van der Et J, Helders PJ, Takken T. High-intensity interval training in an adolescent with cystic fibrosis: a physiological perspective. Physiother Theory Pract 2011;27(3):231-237.

9. Sedentary Behaviour Research Network. Letter to the Editor: Standardized use of the terms "sedentary" and "sedentary behaviours."ApplPhysiol Nutr Metab Physiol 2012;37(3):540-542.

10. Tremblay M, LeBlanc A, Kho M, Saunders T, Larouche R, Colley R, et al. Systematic review of sedentary behaviour and health indicators in school-aged children and youth. Int J Behav Nutr Phys Act 2011;8(1):98.

11. Goldfield GS, Kenny GP, Hadjiyannakis S, Phillips P, Alberga AS, Saunders TJ, et al. Video game playing is independently associated with blood pressure and lipids in overweight and obese adolescents. PLoS One 2011;6(11):e26643. 
12. Goldfield GS, Saunders TJ, Kenny GP, Hadjiyannakis S, Phillips P, Alberga AS, et al. Screen Viewing and Diabetes Risk Factors in Overweight and Obese Adolescents. Am J Prev Med 2013;44(4):S364-S370.

13. Mark AE, Janssen I. Relationship between screen time and metabolic syndrome in adolescents. J Public Health 2008;30(2):153-160.

14. Saunders TJ, Larouche R, Colley RC, Tremblay MS. Acute Sedentary Behaviour and Markers of Cardiometabolic Risk: A Systematic Review of Intervention Studies. J Nutr Metab 2012;2012:1-12.

15. Dunstan DW, Kingwell BA, Larsen R, Healy GN, Cerin E, Hamilton MT, et al. Breaking up prolonged sitting reduces postprandial glucose and insulin responses. Diabetes Care 2012;35(5):976-983.

16. Healy GN, Dunstan DW, Salmon J, Cerin E, Shaw JE, Zimmet PZ, et al. Breaks in sedentary time: beneficial associations with metabolic risk. Diabetes Care 2008;31(4):661-6.

17. Healy GN, Matthews CE, Dunstan DW, Winkler EAH, Owen N. Sedentary time and cardio-metabolic biomarkers in US adults: NHANES 2003-06. Eur Heart J 2011;32(5):590-597.

18. Colley RC, Garriguet D, Janssen I, Saunders TJ, Carson V, Wong S, et al. The association between accelerometer-measured patterns of sedentary time and health risk in children and youth: a cross-sectional study. BMC Public Health 2013;13:200.

19. Mutch WA, Eschun GM, Kowalski SE, Graham MR, Girling LG, Lefevre GR. Biologically variable ventilation prevents deterioration of gas exchange during prolonged anaesthesia. Br J Anaesth 2000;84(2):197-203.

20. Taleb NN. Antifragile: Things That Gain from Disorder.New York, NY, Random House, 2012.

21. West BJ. Where Medicine Went Wrong: Rediscovering the Path to Complexity. Hackensack, NJ, World Scientific Publishing Company, 2006.

22. Mattson MP, Wan R. Beneficial effects of intermittent fasting and caloric restriction on the cardiovascular and cerebrovascular systems. J Nutr Biochem. 2005;16(3):129-137.

23. Brewster JF, Graham MR, Mutch WAC. Convexity, Jensen's inequality and benefits of noisy mechanical ventilation. J R Soc Interface 2005;2(4):393-396.

24. Colley R, Connor Gorber S, Tremblay MS. Quality control and data reduction procedures for accelerometry-derived measures of physical activity. Health Reports 2010;21(1):6369. 
25. Troiano RP, Berrigan D, Dodd KW, Mâsse LC, Tilert T, McDowell M. Physical activity in the United States measured by accelerometer. Med Sci Sports Exerc 2008;40(1):181188.

26. Trost SG, Pate RR, Sallis JF, Freedson PS, Taylor WC, Dowda M, et al. Age and gender differences in objectively measured physical activity in youth. Med Sci Sports Exerc 2002;34(2):350-355.

27. Willett W, Stampfer MJ. Total energy intake: implications for epidemiologic analyses. Am J Epidemiol 1986;124(1):17-27.

28. NHANES - Questionnaires, Datasets, and Related Documentation [article online], 2012. Available from: http://www.cdc.gov/nchs/nhanes/nhanes_questionnaires.htm. Accessed April 16, 2013.

29. Kuczmarski RJ, Ogden CL, Guo SS, Grummer-Strawn LM, Flegal KM, Mei Z, et al. 2000 CDC Growth Charts for the United States: methods and development. Vital Health Stat 2002;(246):1-190.

30. Friedewald WT, Levy RI, Fredrickson DS.Estimation of the Concentration of LowDensity Lipoprotein Cholesterol in Plasma, Without Use of the Preparative Ultracentrifuge. Clin Chem 1972;18(6):499-502.

31. NHANES 2005 - 2006: Plasma Fasting Glucose and Insulin Data Documentation, Codebook, and Frequencies [article online], 2008. Available from: http://www.cdc.gov/nchs/nhanes/nhanes2005-2006/GLU_D.htm. Accessed April 17, 2013.

32. Franks PW, Ekelund U, Brage S, Wong M-Y, Wareham NJ. Does the Association of Habitual Physical Activity With the Metabolic Syndrome Differ by Level of Cardiorespiratory Fitness? Diabetes Care 2004;27(5):1187 -1193.

33. Strøyer J, Hansen L, Klausen K. Physiological profile and activity pattern of young soccer players during match play. Med Sci Sports Exerc 2004;36(1):168-174.

34. Guagliano JM, Rosenkranz RR, Kolt GS. Girls' physical activity levels during organized sports in Australia. Med Sci Sports Exerc. 2013;45(1):116-122.

35. Ridley K, Ainsworth BE, Olds TS. Development of a Compendium of Energy Expenditures for Youth. Int J Behav Nutr Phys Act 2008;5(1):45.

36. Hamilton MT, Hamilton DG, Zderic TW. Role of low energy expenditure and sitting in obesity, metabolic syndrome, type 2 diabetes, and cardiovascular disease. Diabetes 2007;56(11):2655-2667. 
37. Tremblay MS, Colley RC, Saunders TJ, Healy GN, Owen N. Physiological and health implications of a sedentary lifestyle. Appl Physiol Nutr Metab 2010;35(6):725-740.

38. Esliger DW, Tremblay MS, Copeland JL, Barnes JD, Huntington GE, Bassett Jr DR. Physical activity profile of Old Order Amish, Mennonite, and contemporary children. Med Sci Sports Exerc 2010;42(2):296-303.

39. Hebert JR, Clemow L, Pbert L, Ockene IS, Ockene JK. Social Desirability Bias in Dietary Self-Report May Compromise the Validity of Dietary Intake Measures. Int J Epidemiol 1995;24(2):389-398.

40. Hill RJ, Davies PSW. The validity of self-reported energy intake as determined using the doubly labelled water technique. Br J Nutr 2001;85(4):415-430. 


\section{Tables}

Table 4.1 Subject characteristics.

\begin{tabular}{|c|c|}
\hline Variables & Total $(\mathrm{N}=1460)$ \\
\hline Age (years) & $14.9(14.8,15.0)$ \\
\hline \multicolumn{2}{|l|}{$\operatorname{Sex}(\%)$} \\
\hline Male & $51.6(753 / 1460)$ \\
\hline Female & $48.4(707 / 1460)$ \\
\hline \multicolumn{2}{|l|}{ Race $(\%)$} \\
\hline Non-Hispanic white & $23.1(337 / 1460)$ \\
\hline Non-Hispanic black & $35.1(512 / 1460)$ \\
\hline Hispanic & $38.4(561 / 1460)$ \\
\hline Other & $3.4(50 / 1460)$ \\
\hline \multicolumn{2}{|l|}{ Accelerometer Derived Variables* } \\
\hline Total wear time (minutes/day) & $860(849,871)$ \\
\hline Sedentary behavior (minutes/day) & $488(479,498)$ \\
\hline Moderate and vigorous physical activity (minutes/day) & $29(26,32)$ \\
\hline Vigorous physical activity (minutes/day) & $5(3,6)$ \\
\hline Breaks in sedentary time (number/day) & $98(97,100)$ \\
\hline Movement variability (counts/minute) & $276976(269637,284315)$ \\
\hline \multicolumn{2}{|l|}{ Markers of cardiometabolic disease risk } \\
\hline BMI $\left(\mathrm{kg} / \mathrm{m}^{2}\right)$ & $22.6(22.2,23.0)$ \\
\hline Waist Circumference $(\mathrm{cm})$ & $78.9(77.8,79.9)$ \\
\hline Insulin (pmol/L) & $66(60,72)$ \\
\hline Glucose (mmol/L) & $5.05(5.00,5.11)$ \\
\hline Triglycerides (mmol/L) & $0.93(0.86,1.01)$ \\
\hline HDL-Cholesterol (mmol/L) & $1.36(1.34,1.39)$ \\
\hline LDL-Cholesterol (mmol/L) & $2.29(2.20,2.38)$ \\
\hline Systolic Blood Pressure (mmHg) & $108(107,109)$ \\
\hline Diastolic Blood Pressure (mmHg) & $59(58,60)$ \\
\hline
\end{tabular}

Continuous data are presented as mean (95\% confidence interval). Percentages are presented as percent (numerator/denominator).

*Corrected for wear time using the residuals method 
Table 4.2 Physical activity and sedentary behavior across tertiles of movement variability.

\begin{tabular}{|c|c|c|c|c|}
\hline & Tertile 1 & Tertile 2 & Tertile 3 & $p$ for trend \\
\hline \multicolumn{5}{|l|}{ Females } \\
\hline Movement variability (counts/min) & $181354(177470,185239)$ & $242329(238467,246191)$ & $326946(315218,338675)$ & \\
\hline MVPA (mins/day) & $10(8,11)^{*}$ & $19(16,21)^{\dagger}$ & $34(31,37)^{\ddagger}$ & $<0.001$ \\
\hline VPA (mins/day) & $2(0,4)^{*}$ & $2(1,3)^{\dagger}$ & $5(4,6)^{7}$ & $<0.001$ \\
\hline Sedentary time (mins/day) & $550(535,565)^{*}$ & $518(500,536)^{\dagger}$ & $459(439,479)^{\sharp}$ & $<0.001$ \\
\hline Breaks in sedentary time (number/day) & $96(94,97)^{*}$ & $96(95,97)^{*}$ & $99(98,100)^{+}$ & $<0.001$ \\
\hline \multicolumn{5}{|l|}{ Males } \\
\hline Movement variability (counts/min) & $212089(202663,221515)$ & $305792(301729,309855)$ & $414946(401964,427928)$ & \\
\hline MVPA (mins/day) & $20(13,28)^{*}$ & $32(29,36)^{\dagger}$ & $64(56,72)^{:}$ & $<0.001$ \\
\hline VPA (mins/day) & $4(0,8)^{*}$ & $4(3,4)^{*}$ & $11(7,16)^{\frac{5}{5}}$ & $<0.001$ \\
\hline Sedentary time (mins/day) & $530(509,552)^{*}$ & $461(444,478)^{\dagger}$ & $399(380,419)^{\ddagger}$ & $<0.001$ \\
\hline Breaks in sedentary time (number/day) & $97(93,102)^{*}$ & $97(97,99)^{*}$ & $105(101,110)^{\frac{5}{5}}$ & 0.0002 \\
\hline
\end{tabular}

Associations assessed using ANCOVAs with adjustment for age, ethnicity, SES, energy intake and smoking status. Data are presented as mean ( $95 \%$ confidence interval). $n=1460$.

Columns with different superscript symbols are significantly different, $p<0.05$.

Although raw values are displayed in the above table, log transformed MVPA, VPA, and breaks in sedentary time were included in statistical analyses.

MVPA: moderate and vigorous physical activity; VPA: vigorous physical activity 
Table 4.3 Associations between movement variability and markers of cardiometabolic disease risk in participants aged 12-17 years.

\begin{tabular}{|l|c|c|}
\hline Outcomes & Model 1 & Model 2 \\
\hline Females & & \\
\hline Clustered Risk & $-0.063(-0.114,-0.012)^{*}$ & $-0.072(-0.124,-0.020)^{*}$ \\
\hline BMI z-Score & $-0.000(-0.020,0.010)$ & $-0.000(-0.020,0.010)$ \\
\hline Log transformed waist circumference & $-0.001(-0.005,0.002)$ & $-0.003(-0.008,0.001)$ \\
\hline Log transformed insulin & $-0.009(-0.022,0.004)$ & $-0.009(-0.023,0.004)$ \\
\hline Log transformed glucose & $-0.000(-0.002,0.002)$ & $0.000(-0.002,0.002)$ \\
\hline Log transformed triglycerides & $-0.002(-0.016,0.011)$ & $-0.003(-0.017,0.011)$ \\
\hline HDL-Cholesterol (mmol/L) & $0.002(-0.004,0.007)$ & $0.001(-0.005,0.008)$ \\
\hline LDL-Cholesterol (mmol/L) & $0.007(-0.010,0.023)$ & $0.005(-0.010,0.021)$ \\
\hline Systolic Blood Pressure (mmHg) & $-0.261(-0.411,-0.110)^{*}$ & $-0.262(-0.442,-0.080)^{*}$ \\
\hline Diastolic Blood Pressure (mmHg) & $-0.184(-0.348,-0.021)^{*}$ & $-0.102(-0.289,0.086)$ \\
\hline Males & & \\
\hline Clustered Risk & $-0.091(-0.171,-0.011)^{*}$ & $-0.119(-0.193,-0.046)^{*}$ \\
\hline BMI z-Score & $0.010(0.000,0.010)^{*}$ & $0.010(0.000,0.010)^{*}$ \\
\hline Log transformed waist circumference & $-0.001(-0.004,0.001)$ & $-0.001(-0.004,0.001)$ \\
\hline Log transformed insulin & $-0.010(-0.020,0.000)$ & $-0.019(-0.030,-0.008)^{*}$ \\
\hline Log transformed glucose & $-0.000(-0.001,0.001)$ & $-0.001(-0.002,-0.000)^{*}$ \\
\hline Log transformed triglycerides & $-0.007(-0.016,0.001)$ & $-0.004(-0.014,0.006)$ \\
\hline HDL-Cholesterol (mmol/L) & $0.003(-0.001,0.007)$ & $0.004(-0.001,0.010)$ \\
\hline LDL-Cholesterol (mmol/L) & $-0.002(-0.014,0.010)$ & $0.002(-0.016,0.019)$ \\
\hline Systolic Blood Pressure (mmHg) & $-0.108(-0.239,0.023)$ & $-0.171(-0.308,-0.033)^{*}$ \\
\hline Diastolic Blood Pressure (mmHg) & $-0.015(-0.150,0.116)$ & $0.065(-0.140,0.268)$ \\
\hline Associatis asssed using &
\end{tabular}

Associations assessed using linear regression.

Data are presented as the change in outcome associated with a 10000 CPM increase in movement variability (95\% confidence interval).

Model 1: Adjusted for age, ethnicity, SES, kcal, smoking and WC (when not in outcome).

Model 2: Adjusted as above, as well as sedentary behavior and moderate and vigorous physical activity.

Insulin, glucose, triglycerides, and waist circumference were log transformed prior to analyses. Analyses involving BMI, WC and HDL-cholesterol and blood pressure included 707 females and 753 males participants, while all others included 314 females and 342 males.

$*=$ statistically significant, $p<0.05$.

BMI: body mass index; WC: waist circumference; HDL-C: HDL-cholesterol; LDL-C: LDLcholesterol. 


\title{
Chapter 5 - Study 3: Prolonged sitting and markers of cardiometabolic
}

\author{
disease risk in children and youth: a randomized crossover study
}

The following article is in press at the journal Metabolism: Clinical and Experimental, and has been formatted according to their requirements. Data from this article were presented at the International Congress on Physical Activity and Public Health in October, 2012, and at the American College of Sports Medicine AGM in May, 2013.

Authors: Travis J. Saunders ${ }^{1,2}$, Jean-Philippe Chaput ${ }^{1,2}$, Gary S. Goldfield ${ }^{1,2}$, Rachel C. Colley ${ }^{1,2}$, Glen P. Kenny ${ }^{2}$, Eric Doucet ${ }^{2}$, Mark S. Tremblay ${ }^{1,2}$

\section{Affiliations:}

${ }^{1}$ Healthy Active Living and Obesity Research Group, Children's Hospital of Eastern Ontario Research Institute, Ottawa, Ontario, Canada

${ }^{2}$ School of Human Kinetics, University of Ottawa, Ottawa, Ontario, Canada

\section{Author Contributions}

Study design: TJS, JPC, RCC, GSG, GPK, ED, MST; data collection: TJS, JPC, ED; Data analysis and interpretation: TJS, JPC, MST; manuscript writing and editing: TJS, JPC, RCC, GSG, GPK, ED, MST.

\section{Acknowledgements}

This study was supported by a Research Grant from the Children's Hospital of Eastern Ontario Research Institute to JPC. TJS is supported by Doctoral Research Awards from the Canadian Institutes of Health Research and the Canadian Diabetes Association, as well as an Excellence Scholarship from the University of Ottawa.The authors wish to acknowledge the important contribution made to this study by the study participants and their parents. In addition, they wish to thank Natalie Tremblay for aiding with participant recruitment, as well as Ann Beninato, Isabelle Laforest, Mike Borghese, Niko Tzakis, Allana LeBlanc, Joel Barnes and Heather Wright for their assistance with data collection. 


\section{Structured Abstract}

Objective: Recent evidence suggests that short bouts of uninterrupted sedentary behavior reduce insulin sensitivity and glucose tolerance while increasing triglyceride levels in both healthy and overweight/obese adults. To date no study has examined the acute impact of uninterrupted sitting in children and youth. The objective of the present study was to determine whether 8 hours of uninterrupted sitting increase markers of cardiometabolic disease risk in healthy children and youth, in comparison to 8 hours of sitting interrupted by light intensity walk breaks or structured physical activity.

Materials/Methods: 11 healthy males and 8 healthy females between the ages of 10 and 14 years experienced 3 conditions in random order: (1) 8 hours of uninterrupted sitting (Sedentary); (2) 8 hours of sitting interrupted with a 2-minute light-intensity walk break every 20 minutes (Breaks); and (3) 8 hours of sitting interrupted with a 2-minute light-intensity walk break every 20 minutes as well as 2 x 20 minutes of moderate-intensity physical activity (Breaks+Physical Activity).

Insulin, glucose, triglyceride, HDL and LDL cholesterol area under the curve were calculated for each condition.

Results: We observed no significant differences in the area under the curve for any marker of cardiometabolic disease risk across the 3 study conditions (all $p>0.09$ ).

Conclusions: These results suggest that in comparison to interrupted sitting or structured physical activity, a single bout of 8 hours of uninterrupted sitting does not result in measurable changes in circulating levels of insulin, glucose, or lipids in healthy children and youth.

Key Terms: Sedentary behavior, insulin sensitivity, glucose tolerance, pediatric population 


\section{Abbreviations}

BMI: Body mass index

REE: Resting Energy Expenditure

iAUC: Incremental area under the curve

HDL: High density lipoprotein

LDL: Low density lipoprotein 


\section{Introduction}

Prolonged bouts of uninterrupted sedentary behavior (sitting or reclining while expending $\leq 1.5$ metabolic equivalents [1]) result in deleterious changes in insulin sensitivity, glucose tolerance, and plasma triglyceride levels in both healthy and overweight/obese adults [2-8]. Although initial studies in this area focused primarily on long-term bed rest and other restrictive forms of sedentary behavior [8], more recent studies have found that prolonged sitting may also result in significant reductions in insulin sensitivity and glucose tolerance in adult participants [3-5]. Dunstan and colleagues have recently reported that insulin and glucose responses to a standardized meal were elevated by nearly $25 \%$ following 7 hours of uninterrupted sitting in overweight and obese adults, in comparison to sitting with periodic light-intensity walk breaks [5]. Moreover, Stephens et al [4] reported that a single day of sitting reduced insulin action by $39 \%$ among a group of recreationally active young adults.

Despite the recent findings in adults, to date the effects of uninterrupted sitting in the pediatric population remain unexamined. Epidemiological studies have reported consistent associations between sedentary behavior and metabolic dysfunction in children and youth [9-11], suggesting that prolonged sitting may have a measurable health impact in this population. Given that the average child in North America spends more than half their waking hours sitting down [12-14], any cardiometabolic disease risk resulting from uninterrupted sedentary behavior in this age group would be of great public health importance. The objective of the present randomized crossover study was to determine whether 8 hours of uninterrupted sitting would result in increased concentrations of common markers of cardiometabolic disease risk in healthy children and youth, in comparison to a day of sitting interrupted by light intensity walk breaks, with and without structured physical activity. 


\section{Methods}

\section{Participants}

Nineteen healthy children and youth (11 male, 8 female) aged 10-14 years were recruited for this study. There were no limits placed on body weight or physical activity levels prior to study entry. Written consent was obtained from the parents of all participants prior to participation. Oral assent was obtained from participants aged 10-13 years, while participants aged 14 years provided written consent. This study conformed to the ethical standards outlined in the Declaration of Helsinki and was approved by the Research Ethics Boards at the Children's Hospital of Eastern Ontario Research Institute and the University of Ottawa.

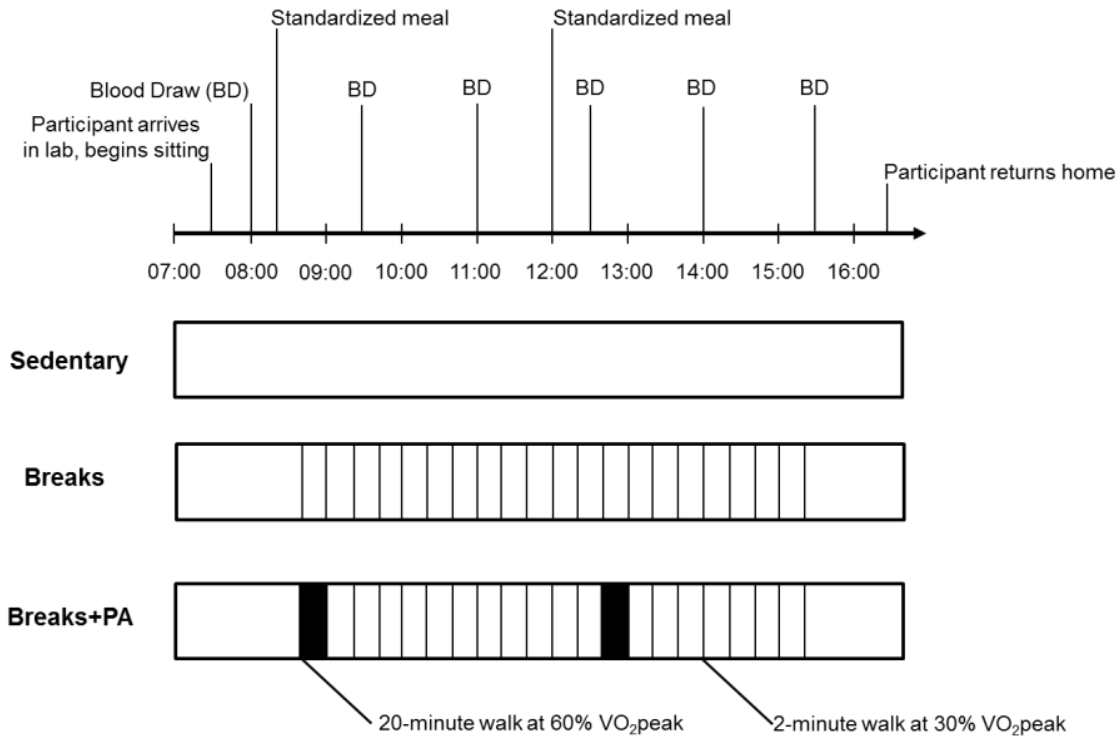

Figure 5.1 Overview of the study protocol.

\section{Baseline Testing Session}

Participants visited the Behavioral and Metabolic Research Unit at the University of Ottawa on 4 occasions - 1 baseline session and 3 experimental sessions - each separated by at least one week. Participants arrived for all sessions at 07:30, and were instructed to fast and abstain from structured exercise for 12 hours prior to each visit. The baseline session included measurements 
related to anthropometry, physical activity, sedentary behavior, cardiorespiratory fitness $\left(\mathrm{VO}_{2}\right.$ peak), and resting energy expenditure (REE). Weight was measured to the nearest $0.1 \mathrm{~kg}$ using a calibrated electronic scale. Standing height was measured to the nearest $0.5 \mathrm{~cm}$ using a wall-mounted stadiometer. Body mass index (BMI) was calculated as weight in kilograms divided by height in meters squared. Waist circumference was measured at the midpoint between the lower border of the last rib and the upper border of the iliac crest after a gentle expiration. Pubertal development was assessed using self-reported Tanner stages as previously validated by Taylor et al. [15].

REE and $\mathrm{VO}_{2}$ peak were assessed using an Ultima PF/PFX (MedGraphics, St Paul, USA) metabolic cart. $\mathrm{VO}_{2}$ peak was measured using the Dubowy graded treadmill protocol [16]. Participants wore an Actical accelerometer (Philips Respironics, Andover, USA) on their right hip for a total of five week days and two weekend days following baseline testing. Accelerometer data were processed using standardized reduction procedures [12] in SAS version 9.2 (SAS Institute, Cary, USA) and were used to assess baseline levels of physical activity and sedentary behavior. Pediatric accelerometer cut-points of 100, 1,500 and 6,500 counts per minute were used to identify light-, moderate- and vigorous-intensity physical activity, respectively [17]. Daily energy requirements were estimated as the sum of REE and average daily physical activityrelated energy expenditure as calculated using the Actical 2.12 software (Philips Respironics, Andover, USA).

\section{Experimental Sessions}

The 3 experimental conditions were performed in random order, as determined by TJS using a random number generator in Microsoft Excel (Microsoft Corporation, Redmond, USA) (Figure 5.1). Participants were blinded to the order of conditions, and only told which condition they 
would experience upon arrival in the lab each morning. Upon arrival to the lab at 07:30 a catheter was inserted into an antecubital vein for blood sampling. During the Sedentary condition, participants remained seated at all times from 07:30 until 15:30 (when necessary, participants were transported to the washroom via wheelchair). The Sedentary With Breaks (Breaks) condition was similar to the Sedentary condition, with the exception that participants walked for 2 minutes on a treadmill at an intensity equivalent to $30 \%$ of $\mathrm{VO}_{2}$ peak every 20 minutes beginning at 8:40 (i.e. 08:40, 09:00, 9:20, etc). Finally, the Sedentary With Breaks and Physical Activity (Breaks+PA) condition was similar to the Breaks condition, but in addition to walking at a lightintensity every 20 minutes, participants also performed two 20-minute bouts of moderateintensity physical activity by walking or jogging on a treadmill at $60 \%$ of $\mathrm{VO}_{2}$ peak from 08:4009:00 and from 12:40-13:00. During all 3 conditions, participants engaged in a standardized set of common sedentary behaviors in identical order -4 hours of watching movies and television programs, 2 hours of puzzles and other forms of mental work, and 1.5 hours of video games.

\section{Standardized Meals}

Standardized meals were provided at breakfast (08:15) and lunch (12:00), using a menu developed for the pediatric population. Breakfast consisted of white bread, butter, peanut butter, cheddar cheese, and orange juice, while lunch included chicken strips, tortilla chips, grapes, baby carrots, $2 \%$ milk, lemonade, ketchup, and Oreo cookies [18]. Both meals were standardized relative to estimated daily energy requirements (rather than macronutrient intake) with breakfast and lunch respectively providing $25 \%$ and $40 \%$ of estimated daily needs. The mean \pm SD intake at breakfast and lunch were $2322 \pm 410$ and $3669 \pm 799 \mathrm{~kJ}$, respectively. The proportion of calories from carbohydrate, fat, and protein respectively at breakfast were $52 \pm 5 \%, 36 \pm 5 \%$ and $12 \pm 1 \%$ while at lunch they were $57 \pm 2 \%, 31 \pm 3 \%$ and $12 \pm 3 \%$. Participants with allergies or food 
intolerances $(n=3)$ had individual food items replaced. However, each participant received identical meals at each of their 3 visits, and was asked to consume all food that was provided.

\section{Markers of Cardiometabolic Disease Risk}

Six blood draws were performed during each experimental day, with each sample requiring approximately $6 \mathrm{ml}$ of blood. The first blood draw occurred at 08:00, and further draws were performed every 90 minutes until 15:30. All markers of cardiometabolic disease risk were assessed in duplicate using heparinized plasma, which was stored at $-80^{\circ} \mathrm{C}$ prior to analysis. There were no missing samples for any participant or variable of interest. Insulin was assayed by enzyme-linked immunosorbent assay (ALPCO Diagnostics, Salem, USA). Glucose, triglycerides, HDL and LDL cholesterol were assessed on the Ortho Vitros 5.1FS (Ortho-Clinical Diagnostics, Rochester, NY). The inter-assay precision for each test was as follows: insulin 11\%; glucose 2\%; triglycerides 2\%; HDL-Cholesterol 3\%, LDL-Cholesterol 3\%. Net incremental area under the curve (iAUC) was calculated for all cardiometabolic disease risk factors using the trapezoid rule [19]. This approach was used rather than the positive iAUC since HDL- and LDLCholesterol curves were expected to have negative values [19].

\section{Statistical Analyses}

Sample size calculations were based on a recent study using a similar crossover design in overweight and obese adults [5]. Although this differs from the current study population, it is the only human study with a design similar in nature to the present study [5]. We estimated that 13 paired observations would provide $90 \%$ power to detect an absolute difference as small as 3,000 $\mathrm{pmol} / \mathrm{L} \cdot \mathrm{min}$ in our primary outcome of insulin incremental area under the curve (iAUC) across conditions with a standard deviation of $3,000 \mathrm{pmol} / \mathrm{L} \cdot \mathrm{min}$ at an alpha level of $p=0.05$ and a twotailed distribution. 
Insulin, triglyceride, HDL and LDL cholesterol iAUC were non-normally distributed, and therefore transformed using a Box-Cox transformation. To determine if males and females could be combined into one analysis, sex-by-condition interactions were assessed for all dependent variables. No significant interactions were detected, therefore males and females were combined for all analyses to maximize statistical power. A linear mixed-model was fitted for the iAUC of each risk factor, with effects for condition, age, sex, BMI, waist circumference, Tanner stage, and baseline physical activity and sedentary behavior. Statistical significance was defined as a $p$ value of 0.05 or less, and a Bonferroni correction was used to adjust for multiple comparisons in post hoc tests following the mixed-effect model. A similar linear mixed-model for raw levels of each risk factor over time was also fitted to assess temporal differences between conditions. This model included effects for condition, time, time-by-condition interaction, age, sex, BMI, waist circumference, Tanner stage, and baseline physical activity and sedentary behavior. Data are presented as mean \pm standard deviation. All statistical tests were performed in SAS 9.2.

\section{Results}

Subject characteristics are presented in Table 5.1. In comparison to female participants, males were significantly older and more sedentary (all $p<0.01)$. However, there were no differences between males and females in terms of BMI, self-reported Tanner stage, moderate-to-vigorous physical activity, or any marker of cardiometabolic disease risk at baseline (all $p \geq 0.15$ ) There were no significant differences in baseline markers of cardiometabolic disease risk across the 3 experimental conditions (all $p>0.25$ ).

iAUC values for the 3 experimental conditions are presented in Table 5.2. We did not observe significant differences for any marker of cardiometabolic disease risk (all $p>0.09$ ). This finding 
remained consistent with or without adjustment for age, sex, Tanner stage, BMI, waist circumference, and baseline physical activity and sedentary behavior. Separating analyses by sex did not materially change these results (data not shown).

When examining temporal changes in markers of cardiometabolic disease risk across conditions, we observed a significant time-by-condition interaction for plasma glucose concentrations only ( $p$ $=0.001)$. Post-hoc tests determined that the glucose concentrations were significantly greater at Time 2 during the Breaks+PA condition than during the Breaks condition $(p=0.004)$ but not the Sedentary condition ( $p=0.051$ ) (Figure 5.2). Glucose levels were not significantly different across conditions at any other time point (all $p>0.40$ ). 


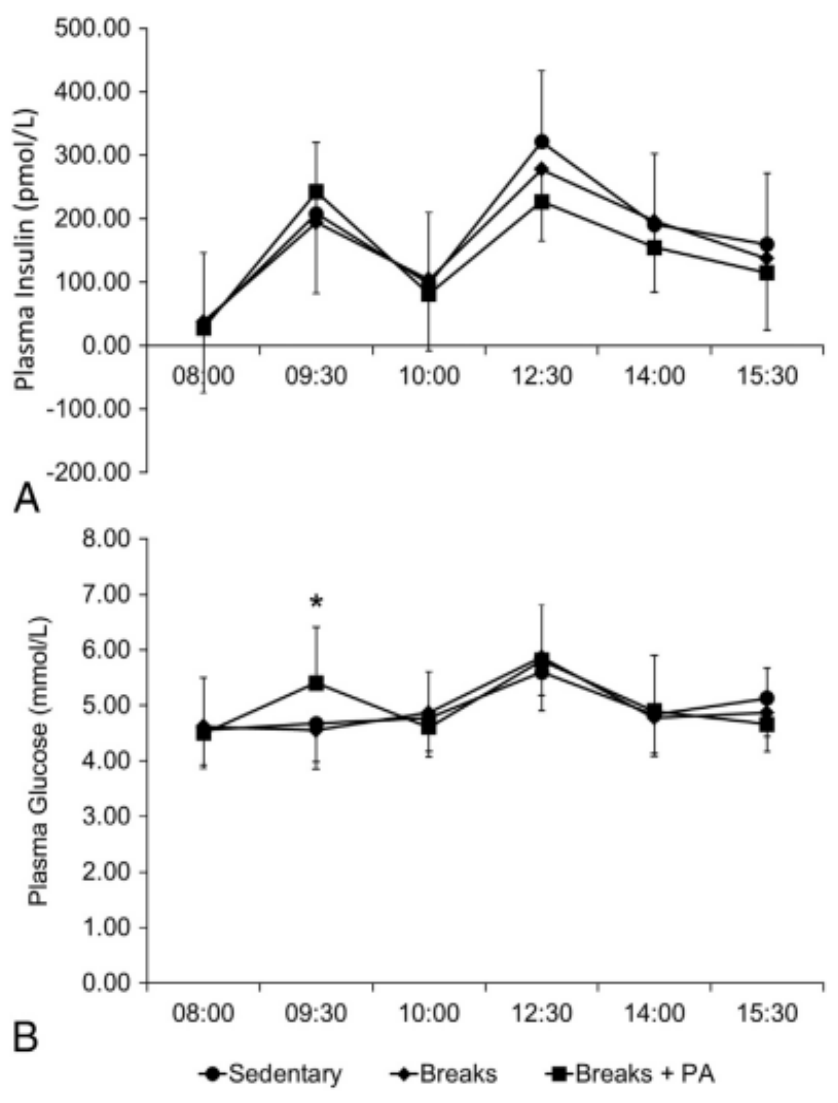

Figure 5.2 Insulin (A) and glucose (B) concentrations during 8 hours of prolonged sitting, with or without interruptions or physical activity $(n=19)$.

Sedentary: 8 hours of uninterrupted sitting.Breaks: 8 hours of sitting interrupted with a 2minute light-intensity walk break every 20 minutes. Breaks + Physical Activity: 8 hours of sitting interrupted with a 2 -minute light-intensity walk break every 20 minutes as well as 40 minutes of moderate-intensity physical activity.

Data are presented as mean and standard deviation. Significance was assessed by a linear mixed-model with effects for condition, time, time-by-condition interaction, age, sex, BMI, waist circumference, Tanner stage, and baseline physical activity and sedentary behavior. A Bonferroni correction was used to adjust for multiple comparisons in post hoc tests following the mixed-effect model. Only plasma glucose showed a significant time-bycondition interaction $(p=0.001) *$ significant difference between Breaks and Breaks + PA condition, $p<0.01$. 


\section{Discussion}

The results of the present randomized crossover study suggest that an acute prolonged bout of uninterrupted sitting does not result in deleterious changes in traditional markers of cardiometabolic disease risk in healthy children and youth. Although we observed a small increase in glucose levels following breakfast in the Breaks+PA condition, we observed no other differences across the three study conditions for any outcome of interest. These results are in contrast to those reported in both healthy and overweight/obese adults, where uninterrupted sitting has been reported to result in acute and deleterious changes in insulin sensitivity and glucose tolerance [5].

There are several factors which could explain the discrepancy between the current findings and those observed in adults [2-5]. The current study focused on healthy boys and girls who were more physically active than the average Canadian youth [12]. Girls, but not boys, were also less sedentary than the national average [12]. The participants were also aerobically fit and metabolically healthy at baseline, which is underscored by the relatively small insulin and glucose responses following both breakfast and lunch. It is probable that a similar investigation in physically inactive youth, a highly sedentary population, or those with obesity or other elevated cardiometabolic disease risk factors, may produce results more similar to those observed in adults. Employing a more sensitive measure of metabolic disease risk (i.e. continuous glucose measurements), or a larger food challenge (i.e. liquid meal high in fat and/or sugar) may also have more clearly differentiated between the study conditions. However, these techniques are substantially more burdensome than those used in the current study, which may impede their use in studies of the pediatric population. Examining the expression of genes related to carbohydrate and lipid metabolism may also have revealed differences between conditions, and is worth exploring in future studies in this population. However, given the present results it seems likely 
that the acute impact of a single bout of uninterrupted sitting on cardiometabolic disease biomarkers is simply smaller (or absent) in the pediatric population, as compared to adults.

It is not immediately clear why a transient increase in plasma glucose levels was observed at 9:30 during the Breaks+PA condition, but not during the Sedentary or Breaks conditions in the present study. It is worth noting that this increase occurred at the blood draw following the first 20minute bout of exercise at $60 \%$ of $\mathrm{VO}_{2}$ peak. Aerobic exercise has been reported to increase hepatic glucose production and plasma glucose levels in adults [20], and it is possible that this was the cause of the increase observed in the present study. However, there is unlikely to be any clinical significance to this brief and relatively small increase in glucose levels.

The current study has several strengths and limitations that warrant mention. This is the first investigation into the acute impact of uninterrupted sedentary behavior in the pediatric population, and employed a rigorous randomized crossover design. Further, in contrast to the liquid meals that are sometimes used in adult studies of this nature [5], the standardized meals employed in the current study were similar to the food eaten by children on a normal basis, increasing the ecological validity of our study. However, it is also possible that a liquid meal that is high in sugar and fat may also have provided a greater metabolic stimulus, which may have more effectively differentiated the impact of our three study conditions. A longer exposure to uninterrupted sitting may also have resulted in different results, although the ecological validity of such an approach would be questionable. All participants in the current study were healthy at baseline, and the majority were both lean and physically active. It is therefore unclear whether similar results would be observed among a population of overweight or obese youth, or those showing signs of metabolic dysfunction. The present findings are also limited by our small sample size, and by the relatively small number of outcomes which were examined. Finally, the 
present study did not examine whether fluctuations in energy balance across the three conditions may have influenced metabolic risk [4].

It is possible that the present findings may have differed had our study included a large number of participants, although our sample size calculation suggests that we had sufficient power for our primary outcome of insulin iAUC [5]. It is also worth noting that there were no consistent trends across the various outcomes measured, regardless of statistical significance. Although the insulin iAUC during the Sedentary condition was the highest of the 3 conditions, this was not the case for any other risk marker. This lends support to our conclusion that prolonged sitting does not result in significant increases in markers of cardiometabolic disease risk in this age group, and suggests that our results would not have been appreciably different with a larger sample size.

In conclusion, our findings suggest that in comparison to light walk breaks with or without structured physical activity, 8 hours of uninterrupted sitting do not result in measurable changes in circulating levels of insulin, glucose, or lipids in healthy children and youth. This suggests that the relationship between sedentary behavior and increased health risk observed in epidemiological studies may be due to the behaviors children engage in while seated, rather than any direct metabolic impact of sitting per se [21-23]. Future research should involve children at risk of cardiometabolic abnormalities to determine whether the results are comparable. 


\section{References}

1. Sedentary Behaviour Research Network. Letter to the Editor: Standardized use of the terms "sedentary" and "sedentary behaviours". Appl Physiol Nutr Metab 2012;37: 540542; doi:10.1139/h2012-024

2. Saunders TJ, Larouche R, Colley RC, et al. Acute Sedentary Behaviour and Markers of Cardiometabolic Risk: A Systematic Review of Intervention Studies. J Nutr Metab 2012: 1-12; doi:10.1155/2012/712435

3. Duvivier BMFM, Schaper NC, Bremers MA, et al. Minimal Intensity Physical Activity (Standing and Walking) of Longer Duration Improves Insulin Action and Plasma Lipids More than Shorter Periods of Moderate to Vigorous Exercise (Cycling) in Sedentary Subjects When Energy Expenditure Is Comparable. PLoS ONE 2013;8: e55542; doi:10.1371/journal.pone.0055542

4. Stephens BR, Granados K, Zderic TW, et al. Effects of 1 day of inactivity on insulin action in healthy men and women: interaction with energy intake. Metabolism 2011;60: 941-949.

5. Dunstan DW, Kingwell BA, Larsen R, et al. Breaking up prolonged sitting reduces postprandial glucose and insulin responses. Diabetes Care 2012; 35: 976-983.

6. Nygaard H, Tomten SE, Høstmark AT. Slow postmeal walking reduces postprandial glycemia in middle-aged women. Appl Physiol Nutr Metab 2009; 34: 1087-1092; doi:10.1139/H09-110

7. Yaroshenko YY, Zorban YG, Kuznetsov NK, et al. Changes in thyroid hormones and lipids in endurance trained volunteers during acute and rigorous bed rest conditions. Wiener Klinische Wochenschrift 1998;110: 225-231.

8. Bergouignan A, Rudwill F, Simon C, et al. Physical inactivity as the culprit of metabolic inflexibility: evidence from bed-rest studies. J Appl Physiol 2011;111: 1201-1210. 
9. Tremblay M, LeBlanc A, Kho M, et al. Systematic review of sedentary behaviour and health indicators in school-aged children and youth.Int J Behav Nutr Phys Act 2011; 8: 98; doi:10.1186/1479-5868-8-98

10. Goldfield GS, Kenny GP, Hadjiyannakis S, et al. Video game playing is independently associated with blood pressure and lipids in overweight and obese adolescents. PloS ONE 2011;6: e26643.

11. Goldfield GS, Saunders TJ, Kenny GP, et al. Screen Viewing and Diabetes Risk Factors in Overweight and Obese Adolescents. Am J Prev Med 2013; 44: S364-S370; doi:10.1016/j.amepre.2012.11.040

12. Colley R, Garriguet D, Janssen I, et al. Physical activity of Canadian children and youth: accelerometer results from the 2007 to 2009 Canadian Health Measures Survey. Health Reports 2011;22: 15-24.

13. Pate RR, Mitchell JA, Byun W, et al. Sedentary behaviour in youth. Br J Sports Med 2011;45: 906-913; doi:10.1136/bjsports-2011-090192

14. Leatherdale ST, Ahmed R. Screen-based sedentary behaviours among a nationally representative sample of youth: are Canadian kids couch potatoes? Chronic Dis Inj Can 2011;31: 141-146.

15. Taylor SJ, Whincup PH, Hindmarsh PC, et al. Performance of a new pubertal selfassessment questionnaire: a preliminary study. Paediatr Perinat Epidemiol 2011;15: 8894.

16. Dubowy K-O, Baden W, Bernitzki S, et al. A practical and transferable new protocol for treadmill testing of children and adults.Cardiology in the Young 2008;18: 615-623; doi:10.1017/S1047951108003181

17. Puyau MR, Adolph AL, Vohra FA, et al. Prediction of activity energy expenditure using accelerometers in children. Med Sci Sports Exerc 2004;36: 1625-1631. 
18. Shomaker LB, Tanofsky-Kraff M, Zocca JM, et al. Eating in the absence of hunger in adolescents: intake after a large-array meal compared with that after a standardized meal. Am J Clin Nutr 2010;92: 697-703.

19. Wolever TMS. Effect of blood sampling schedule and method of calculating the area under the curve on validity and precision of glycaemic index values.Br J Nutr 2004;91: $295-300$.

20. Howlett K, Febbraio M, Hargreaves M. Glucose production during strenuous exercise in humans: role of epinephrine. Am J Physiol Endocrinol Metab 1999;276: E1130-E1135.

21. Chaput JP, Saunders TJ, Mathieu ME, et al. Combined associations between moderate to vigorous physical activity and sedentary behavior with cardiometabolic risk factors in children. Appl Physiol Nutr Metab In Press; doi:10.1139/apnm-2012-0382.

22. Saunders TJ, Chaput JP. Is obesity prevention as simple as turning off the television and having a nap? Br J Nutr 2012;108: 946-947; doi:10.1017/S0007114512002644

23. Thivel D, Aucouturier J, Doucet E, et al. Daily energy balance in children and adolescents. Does energy expenditure predict subsequent energy intake? Appetite 2013; 60: 58-64; doi:10.1016/j.appet.2012.09.022 
Tables

Table 5.1 Participant characteristics.

\begin{tabular}{|c|c|c|c|}
\hline & Male $(\mathrm{n}=11)$ & Female $(\mathrm{n}=8)$ & $p$ value \\
\hline Age (years) & $12.9(0 / 8)$ & $11.3(0.7)$ & $<0.01$ \\
\hline BMI $\left(\mathrm{kg} / \mathrm{m}^{2}\right)$ & $18.7(4.5)$ & $17.4(2.9)$ & 0.49 \\
\hline Waist Circumference (cm) & $66.6(15.8)$ & $59.8(5.7)$ & 0.26 \\
\hline Tanner Stage & $1.9(1.0)$ & $1.5(0.8)$ & 0.41 \\
\hline Sedentary behavior (min/day) & $539.4(48.3)$ & $461.1(66.0)$ & $<0.01$ \\
\hline MVPA (min/day) & $66.8(28.5)$ & $59.5(23.8)$ & 0.56 \\
\hline Insulin (pmol/L) & $42.6(27.7)$ & $33.1(18.8)$ & 0.42 \\
\hline Glucose (mmol/L) & $4.7(0.4)$ & $4.6(0.3)$ & 0.71 \\
\hline LDL-Cholesterol (mmol/L) & $1.9(0.6)$ & $2.0(0.7)$ & 0.91 \\
\hline HDL-Cholesterol (mmol/L) & $1.3(0.3)$ & $1.3(0.4)$ & 0.15 \\
\hline Triglycerides (mmol/L) & $0.7(0.2)$ & $0.7(0.2)$ & 0.81 \\
\hline
\end{tabular}

BMI: body mass index; MVPA: moderate-and-vigorous physical activity; LDL: low density lipoprotein; HDL: high density lipoprotein. Date are presented as mean (SD). Significance was assessed using an independent measures $t$ test. 
Table 5.2 Net incremental area under the curve (iAUC) values for biomarkers of cardiometabolic disease risk during 8 hours of prolonged sitting, with or without interruptions or physical activity (n=19).

\begin{tabular}{|c|c|c|c|c|}
\hline & Sedentary & Breaks & Breaks + Physical Activity & $p$ for trend \\
\hline Insulin (pmol/L·min) & $80,559.3(66380.3)$ & $78,707.3(83074.5)$ & $64,270.8(42272.4)$ & 0.552 \\
\hline Glucose (mmol/L·min) & $185.3(171.7)$ & $163.2(148.8)$ & $248.0(220.4)$ & 0.091 \\
\hline Triglycerides (mmol/L·min) & $99.8(114.4)$ & $101.5(69.0)$ & $65.2(66.4)$ & 0.106 \\
\hline HDL-Cholesterol (mmol/L·min) & $-38.7(39.9)$ & $-32.2(40.2)$ & $-52.3(41.4)$ & 0.431 \\
\hline LDL-Cholesterol (mmol/L·min) & $-62.9(67.2)$ & $-50.0(28.9)$ & $-76.5(51.4)$ & 0.400 \\
\hline
\end{tabular}

Sedentary: 8 hours of uninterrupted sitting.

Breaks: 8 hours of sitting interrupted with a 2-minute light-intensity walk break every 20 minutes.

Breaks + Physical Activity: 8 hours of sitting interrupted with a 2-minute light-intensity walk break every 20 minutes as well as 40 minutes of moderate-intensity physical activity.

Data are presented as mean (standard deviation). Significance was assessed by a linear mixed-model with effects for condition, age, sex,

BMI, waist circumference, Tanner stage, and baseline physical activity and sedentary behavior. Although raw values are presented above,

all statistical analyses have been performed using normalized data. 


\section{Chapter 6 - Study 4: Children and youth do not compensate for an imposed bout of prolonged sitting by reducing subsequent food intake or increasing physical activity: a randomized crossover study}

The following article has been accepted for publication in the British Journal of Nutrition (Cambridge University Press), and has been formatted according to their requirements. Copyright of this article remains with the authors. Data from this article were presented at the American College of Sports Medicine AGM in May, 2013.

Authors: Travis J. Saunders ${ }^{1,2}$, Jean-Philippe Chaput ${ }^{1,2}$, Gary S. Goldfield ${ }^{1,2}$, Rachel C. Colley ${ }^{1,2}$, Glen P. Kenny ${ }^{2}$, Eric Doucet ${ }^{2}$, Mark S. Tremblay ${ }^{1,2}$

\section{Affiliations:}

${ }^{1}$ Healthy Active Living and Obesity Research Group, Children's Hospital of Eastern Ontario Research Institute, Ottawa, Ontario, Canada

${ }^{2}$ School of Human Kinetics, University of Ottawa, Ottawa, Ontario, Canada

\section{Author Contributions}

All authors contributed to the design of the research project; research was conducted by TJS and JPC; lab space and equipment were contributed by ED; TJS performed statistical analyses, and wrote the first draft of the manuscript with JPC and MST; all authors provided critical feedback to the manuscript and approved the final version; TJS takes primary responsibility for manuscript content. The authors have no potential conflicts of interest to report.

\section{Acknowledgements}

The authors wish to acknowledge the important contribution made to this study by the study participants and their parents. In addition, they wish to thank Natalie Tremblay for aiding with participant recruitment, Kathryn Williams for her help with statistical analyses, as well as Ann Beninato, Isabelle Laforest, Mike Borghese, Niko Tzakis, Allana LeBlanc and Joel Barnes for their assistance with data collection. This study was supported by a Research Grant from the 
Children's Hospital of Eastern Ontario Research Institute to JPC. TJS is supported by Doctoral Research Awards from the Canadian Institutes of Health Research and the Canadian Diabetes Association, as well as an Excellence Scholarship from the University of Ottawa. 


\begin{abstract}
The behavioural impact of an imposed bout of prolonged sitting has yet to be investigated in the pediatric population. Our objective was to determine the acute effect of prolonged sitting on $a d$ libitum food intake and spontaneous physical activity in healthy children and youth. A total of 20 healthy youth (12 males, 8 females) aged 10-14 years, with a mean \pm SD BMI of $18.6 \pm 4.3 \mathrm{~kg} / \mathrm{m}^{2}$, experienced 3 conditions in random order: (1) a day of uninterrupted sitting (Sedentary); (2) a day of sitting interrupted with a 2-minute light-intensity walk break every 20 minutes (Breaks); and (3) a day of sitting interrupted with a 2-minute light-intensity walk break every 20 minutes as well as 2 × 20 minutes of moderate-intensity physical activity (Breaks $+P A)$. Food intake (ad libitum buffet meal) and physical activity (accelerometry for 24 hours) were assessed following each condition. Despite significant differences in sedentary behaviour and activity levels during the 3 in-lab sessions (all $p<0.01$ ), we observed no differences in ad libitum food intake immediately following each condition, nor any changes in the level of sedentary behaviour or physical activity in the 24-hours following each condition (all $p>0.25$ ). These findings suggest that children and youth may not compensate for an imposed bout of sedentary behaviour by reducing subsequent food intake or increasing physical activity.
\end{abstract}




\section{Introduction}

Both acute and chronic exposure to some sedentary behaviours (activities that involve sitting or reclining while expending $\leq 1.5$ metabolic equivalents ${ }^{(1)}$ ) have been associated with excess food intake and weight gain in children and youth ${ }^{(2-5)}$. Chaput et al. ${ }^{(2)}$ reported that in comparison to seated rest, 45 minutes of seated video game play resulted in significant increases in acute food intake and positive energy balance in adolescent males. Similarly, a recent systematic review by Tremblay and colleagues ${ }^{(3)}$ concluded that sedentary behaviour (generally measured as time spent watching TV) was consistently associated with increased body weight and other markers of adiposity among school-aged children. This evidence has led some to suggest that sedentary behaviour may be a key contributor to increasing pediatric obesity rates ${ }^{(6-8)}$. However, while there is evidence that some common modalities of sedentary behaviour are likely to increase energy intake in children and youth, the impact of sitting per se has yet to be investigated ${ }^{(6)}$.

The influence of an imposed bout of prolonged sitting on subsequent physical activity in children and youth is also unclear. It has previously been suggested that physical activity levels among this population are regulated by an "activitystat"(9-11). In support of this view, several reports suggest that in response to an imposed bout of physical activity, youth may consciously or unconsciously compensate by reducing their physical activity levels throughout the rest of the day ${ }^{(9-12)}$. However, no study has yet examined whether an imposed bout of sedentary behaviour (i.e. sitting) results in a similar behavioural compensation in free-living conditions. If activity levels are regulated by a central mechanism similar to the "activitystat", it is plausible that youth may compensate for a prolonged period of sitting or inactivity by reducing their level of sedentary behaviour and increasing their level of physical activity later in the day. Given that North American children spend most of their waking time engaging in sedentary behaviours ${ }^{(13-15)}$, it is pertinent to investigate the impact of prolonged sitting on subsequent food intake and physical activity, both of which are important health-related behaviours.

The objective of this randomized crossover study was to determine whether one day of uninterrupted sitting would result in different compensatory changes in ad libitum food intake and/or spontaneous physical activity in healthy children and youth, in comparison to a day of sitting interrupted by light intensity walk breaks, with and without structured physical activity. Based on the available evidence, we hypothesized that prolonged sitting would result in a compensatory increase in subsequent spontaneous physical activity, a reduction in sedentary behaviour, and no change in ad libitum food intake. 


\section{Experimental Methods}

\section{Subjects}

Twenty healthy children and youth (12 males, 8 females) aged 10-14 years participated in this intervention study. There were no limits placed on participant weight or activity levels. This study was conducted according to the guidelines laid down in the Declaration of Helsinki and all procedures involving human subjects/patients were approved by the institutional Research Ethics Boards at the Children's Hospital of Eastern Ontario and the University of Ottawa. Written informed consent was obtained from the parents of all participants. Oral assent was obtained from participants aged 10-13 years (assent was witnessed and formally recorded), while participants aged 14 years provided written consent prior to participation.

\section{Baseline Testing Session}

The current analysis is part of a larger study examining the metabolic impact of prolonged sitting in children and youth, which has been described previously ${ }^{(16)}$. Participants attended 1 baseline session and 3 experimental sessions, each separated by at least one week. All sessions began at 07:30, and participants were instructed to fast and abstain from structured exercise for 12 hours prior to each visit. The baseline session included measurements related to anthropometry, physical activity, sedentary behaviour, cardiorespiratory fitness ( $\mathrm{VO}_{2}$ peak), and resting energy expenditure (REE). At this initial visit participants were asked to identify any food allergies or intolerances that might impact the standardized breakfast and buffet meals during the experimental sessions. Weight was measured to the nearest $0.1 \mathrm{~kg}$ using a BWB-800AS calibrated electronic scale (Tanita Corporation of America Inc., Arlington Heights, IL). Standing height was measured to the nearest $0.5 \mathrm{~cm}$ using a Tanita HR-100 wall-mounted stadiometer (Tanita Corporation of America Inc., Arlington Heights, IL). Body mass index (BMI) was calculated as weight divided by height squared $\left(\mathrm{kg} / \mathrm{m}^{2}\right)$. Children were categorized as overweight/obese using the International Obesity Taskforce (IOTF) cut-points. ${ }^{(17)}$ Waist circumference was measured at the midpoint between the lower border of the last rib and the upper border of the iliac crest after a gentle expiration. Pubertal development was assessed using self-reported Tanner stages as previously validated by Taylor et ${ }^{(18)}$.

REE and $\mathrm{VO}_{2}$ peak were measured using an Ultima PF/PFX (MedGraphics, St Paul, USA) metabolic cart. $\mathrm{VO}_{2}$ peak was assessed using the Dubowy graded treadmill protocol ${ }^{(19)}$. Participants wore an Actical accelerometer (Philips Respironics, Andover, USA) on their right hip 
for seven consecutive days following baseline testing. Accelerometer data were processed using standardized reduction procedures ${ }^{(13)}$ in SAS version 9.2 (SAS Institute, Cary, USA) and used to assess baseline levels of physical activity and sedentary behaviour. Accelerometer cut-points of 100, 1500 and 6500 counts per minute were used to identify light-, moderate-, and vigorousintensity physical activity, respectively ${ }^{(20)}$. Total energy expenditure during each of the experimental conditions was estimated using the following formula, where the thermic effect of food is fixed at 10\%: (REE + physical activity energy expenditure during the session) $\times 1.11^{(21)}$.

\section{Experimental Sessions}

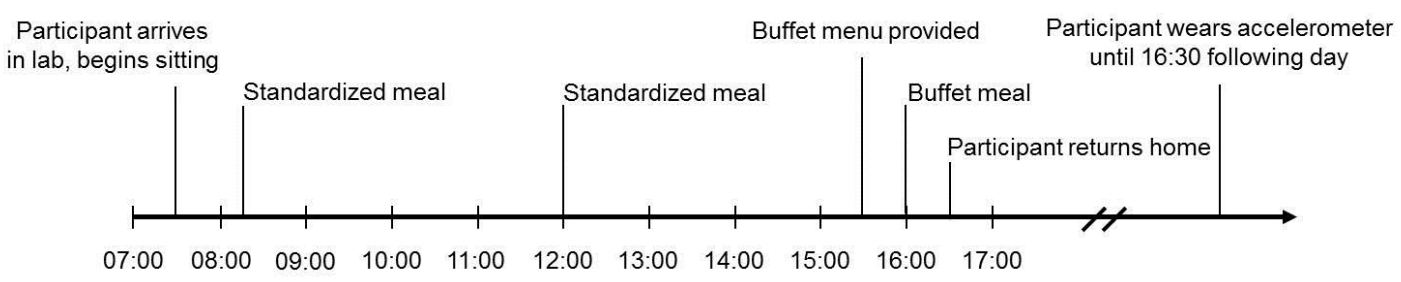

Sedentary

Breaks

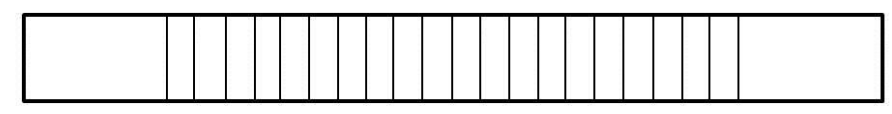

Breaks+PA

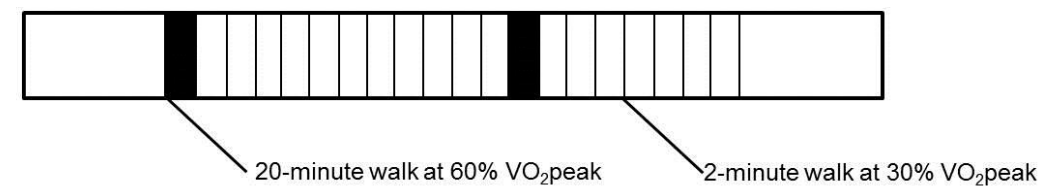

Figure 6.1 Overview of the study protocol (modified from Saunders et al. ${ }^{(16)}$ ).

Sedentary: a day of uninterrupted sitting.

Breaks: a day of sitting interrupted with a 2-minute light-intensity walk break every 20 minutes.

Breaks + Physical Activity: a day of sitting interrupted with a 2-minute light-intensity walk break every 20 minutes as well as $\mathbf{4 0}$ minutes of moderate-intensity physical activity.

The 3 experimental conditions were performed in random order, as determined using a random number generator in Microsoft Excel (Microsoft Corporation, Redmond, USA). Participants arrived at the lab at 07:30 for all experimental conditions and began sitting. During the Sedentary condition, participants remained seated without interruption until 16:30 (when necessary, participants were transported to the washroom via wheelchair) (Figure 6.1). The Sedentary With 
Breaks (Breaks) condition was similar to the Sedentary condition, with the exception that participants walked for 2 minutes on a treadmill at an intensity equivalent to $30 \%$ of $\mathrm{VO}_{2}$ peak every 20 minutes beginning at 08:40 (i.e. 08:40, 09:00, 09:20, etc). Finally, the Sedentary With Breaks and Physical Activity (Breaks $+P A$ ) condition was similar to the Breaks condition, but in addition to walking at a light-intensity every 20 minutes, participants also performed two 20minute bouts of moderate-intensity physical activity by walking or jogging on a treadmill at $60 \%$ of $\mathrm{VO}_{2}$ peak from 08:40-09:00 and from 12:40-13:00.

During all 3 conditions, participants engaged in a standardized set of common sedentary behaviours in identical order -4 hours of watching movies and television programs, 2 hours of puzzles and other forms of mental work, and 2 hours of video games. Each experimental condition concluded with a buffet meal which lasted from 16:00-16:30. Participants wore accelerometers for the duration of each experimental condition and the 24-hours following each condition to assess levels of physical activity and sedentary behaviour.

\section{Standardized Meals}

Standardized meals were provided at breakfast (08:15) and lunch (12:00), using a menu developed for the pediatric population ${ }^{(22)}$. Breakfast consisted of white bread, butter, peanut butter, cheddar cheese, and orange juice, while lunch included chicken strips, tortilla chips, grapes, baby carrots, $2 \%$ milk, lemonade, ketchup, and Oreo cookies. Both meals were standardized relative to estimated daily energy requirements (rather than macronutrient intake) with breakfast and lunch respectively providing $25 \%$ and $40 \%$ of estimated daily needs. Daily energy requirements were estimated as the sum of REE and average daily physical activity-related energy expenditure recorded at baseline. The mean \pm SD intake at breakfast and lunch were $2322 \pm 410$ and $3669 \pm 799 \mathrm{~kJ}$, respectively. The proportion of kilojoules from carbohydrate, fat, and protein respectively at breakfast were $52 \pm 5 \%, 36 \pm 5 \%$ and $12 \pm 1 \%$ while at lunch they were $57 \pm 2 \%, 31 \pm 3 \%$ and $12 \pm 3 \%$. Participants with allergies or food intolerances $(n=3)$ had individual food items replaced. However, each participant received identical meals at each of their 3 visits, and was asked to consume all food that was provided.

\section{Visual Analog Scales (VAS)}

Hunger and prospective food consumption were assessed immediately before participants were provided with the buffet food menu at 15:30 and again immediately following the buffet meal which occurred from 16:00-16:30. This was done using $100 \mathrm{~mm}$ Visual Analog Scales (VAS) 
adapted from those described by Hill and Blundell ${ }^{(23)}$, which are reliable both before and after a

meal $^{(24)}$ and have been employed previously in pediatric populations ${ }^{(2,25)}$. Subjects were asked to place a mark at the position which approximated their level of hunger and the amount of food they thought they could eat at that time.

\section{Buffet Meals}

Spontaneous food intake was assessed using an ad libitum buffet meal at 16:00 during each experimental condition. The buffet has been validated previously ${ }^{(26)}$, and allowed for assessment of total energy intake as well as macronutrient composition. The meal consisted of a variety of foods differing in macronutrient composition. Participants selected items from a written menu, were instructed to eat ad libitum, and were provided with additional servings on request. Participants were given 30 minutes for this meal, and all foods were weighed to the nearest $0.1 \mathrm{~g}$ before and after ingestion. Energy and macronutrient intake were calculated using The Food Processor (ESHA Research, Salem, Oregon).

\section{Statistical Analyses}

As described above, the current analysis is part of a larger investigation of the metabolic impact of prolonged sitting in the pediatric population ${ }^{(16)}$. The primary outcome of the study was insulin sensitivity, which was used to estimate the necessary sample size to assess significance. The sample size for the present analyses was therefore predetermined. However, given the levels of variability observed in the present study, a post hoc sample size calculation revealed that we had greater than $80 \%$ power to detect a difference of 12 minutes/day in moderate physical activity, 5 minutes/day in vigorous physical activity, or $600 \mathrm{~kJ}$ in energy intake across study conditions.

Buffet food intake (both in kilojoules and grams), absolute protein intake, percent fat intake, and both VAS following the buffet meal were non-normally distributed, and were transformed using Box-Cox transformations to improve normality. Baseline differences between male and female participants were assessed by independent samples t test for continuous variables and by chi square test for proportions.

To determine if males and females could be combined in subsequent analyses, sex-by-condition interactions were assessed for all dependent variables. No significant interactions were detected, therefore males and females were combined for all analyses to maximize statistical power and improve clarity. A linear mixed-model was fitted for each food intake-related outcome, with 
effects for condition, age, sex, Tanner stage, BMI and baseline physical activity and sedentary behaviour. Similar models were used for physical activity and sedentary behaviour-related outcomes, with additional adjustment for accelerometer wear-time. This study was not sufficiently powered to investigate the impact of BMI on these results, and therefore BMI-bycondition interactions were not examined. Statistical significance was defined as a two-sided alpha level of 0.05 , and a Bonferroni correction was used to adjust for multiple comparisons in post hoc tests following the mixed-effect model. Data are presented as mean (SD). All statistical tests were performed in SAS 9.2.

\section{Results}

Participant characteristics are presented in Table 6.1. In comparison to their female counterparts, male participants were significantly older, spent more time engaging in sedentary behaviour, and less time engaging in light-intensity physical activity at baseline (all $p<0.03$ ). In contrast, at baseline there were no differences between males and females with respect to BMI, waist circumference, self-reported Tanner stage, or daily moderate-and-vigorous intensity physical activity (MVPA) (all $p>0.15)$.

The amounts of sedentary behaviour, light-, and moderate-intensity physical activity accumulated during each experimental condition are presented in Table 6.2. As imposed, the 3 conditions varied significantly with respect to sedentary time, light- and moderate-intensity physical activity and total steps during the in-lab portion of the study (all $p<0.01$ ). According to accelerometer data, during the Sedentary condition participants spent $97.1 \%$ of lab time engaging in sedentary behaviour, compared to $86.5 \%$ and $81.0 \%$ in the Breaks and Breaks $+P A$ conditions, respectively. As expected, there were no differences in vigorous physical activity across the three study conditions $(p=0.18)$, nor did we observe differences for any measure related to hunger, food intake, or satiety across the three study conditions during the in-lab portion of the study (all $p>0.06$ ) (Table 6.2 and Figure 6.2). These results were similar with and without adjusting for age, sex, Tanner stage, BMI and baseline physical activity and sedentary behaviour. The estimated energy expenditure during the in-lab portion of the study differed significantly across the 3 conditions (all $p<0.01$ ), and is presented with energy intake in Figure 2.

The volume of sedentary behaviour and physical activity accumulated during the 24-hour period immediately following each experimental condition is presented in Table 6.3. We observed no significant differences for any activity-related variable (all $p>0.25$ ). These results were consistent 
whether examining absolute levels of activity, as a percent of total wear-time, as a change score relative to baseline levels, or restricting analyses to only those participants who had 10 or more hours of wear time (data not shown). These results were not impacted by adjustment for age, sex, Tanner stage, BMI, baseline physical activity and sedentary behaviour or accelerometer weartime.

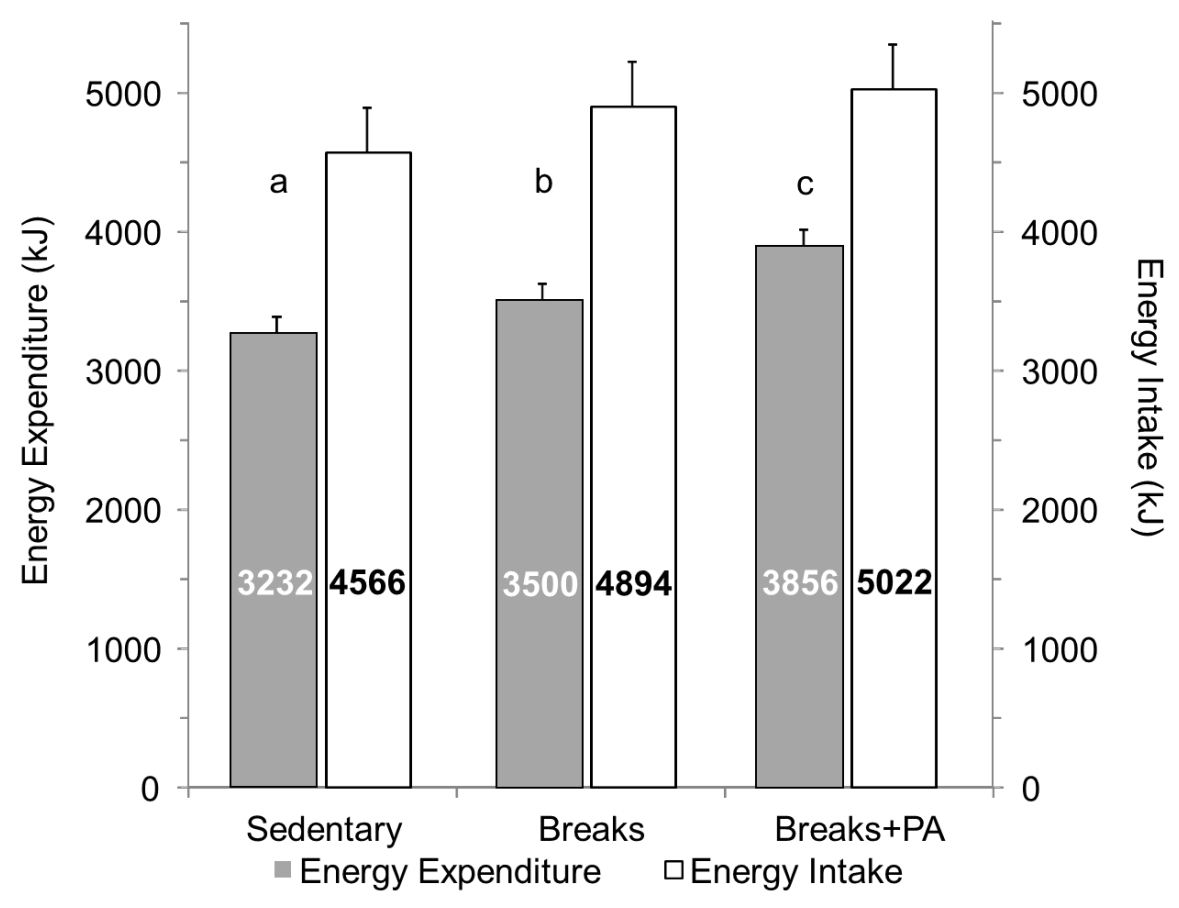

Figure 6.2 Energy intake and estimated energy expenditure while in lab during a day of sitting with or without interruptions and structured physical activity.

Sedentary: a day of uninterrupted sitting.

Breaks: a day of sitting interrupted with a 2-minute light-intensity walk break every 20 minutes.

Breaks + Physical Activity: a day of sitting interrupted with a 2-minute light-intensity walk break every 20 minutes as well as $\mathbf{4 0}$ minutes of moderate-intensity physical activity. Energy intake was assessed using an ad libitum buffet meal, while energy expenditure was estimated as (REE + Physical activity energy expenditure) $x$ 1.11.

Data are presented as mean \pm SEM. Significance was assessed by a linear mixed-model with effects for condition, accelerometer wear time, age, sex, Tanner stage, BMI and baseline physical activity and sedentary behaviour. Bars with different superscript letters are significantly different at $\boldsymbol{p}<\mathbf{0 . 0 5}$ level with Bonferroni correction. 


\section{Discussion}

The findings of the present study, although exploratory and hypothesis generating, suggest that children may not compensate for an acute bout of prolonged sitting by reducing subsequent food intake or increasing physical activity levels. Although there were differences in the level of sedentary behaviour, physical activity, and estimated energy expenditure during the 3 study conditions, we observed no differences in ad libitum food intake immediately following each session, nor were there any differences in physical activity or sedentary behaviour levels in the subsequent 24-hour period. Future studies are needed to examine whether prolonged sitting results in sustained positive energy balance, or whether subsequent adaptations in energy intake or expenditure are able to maintain energy homeostasis.

These results suggest that it is the behaviours that youth commonly engage in while seated (e.g. watching television $^{(4)}$, playing video games ${ }^{(2)}$, or doing mental work ${ }^{(27)}$ ), rather than sitting per se, that result in the increased food intake associated with sedentary behaviour. This is supported by the work of Epstein and colleagues ${ }^{(28-30)}$, who have reported that reductions in screen-based sedentary behaviours have an important influence on both energy intake and body weight among children and youth. For example, Epstein et al. reported that reducing daily screen time by 25 $50 \%$ resulted in a spontaneous reduction in energy intake of $1938 \mathrm{~kJ} /$ day in a group of nonoverweight teens over a 3 week period. ${ }^{(28)}$ Although physical activity-related energy expenditure also increased following the reduction in screen time, it was of a much smaller magnitude than the reduction in energy intake (474 kJ/day). ${ }^{(28)}$ Collectively, these findings suggest that focusing on a reduction in screen-based sedentary behaviours may have a greater impact on energy balance than a similar focus on total sedentary time.

The present findings also support the assertion that energy intake is not acutely coupled with energy expenditure in the pediatric population ${ }^{(6,31)}$. Instead, the available evidence suggests that any acute influence of physical activity on food intake in children and youth is likely to be related to the intensity of the activity, rather than the associated energy expenditure. For example, Thivel and colleagues ${ }^{(12)}$ recently compared the impact of high- $\left(75 \% \mathrm{VO}_{2} \mathrm{max}\right)$ and low-intensity $(40 \%$ $\mathrm{VO}_{2} \max$ ) exercise on ad libitum food intake in obese adolescents. They reported that despite both activity bouts expending roughly $1400 \mathrm{~kJ}$ of energy, only the high-intensity bout reduced subsequent food intake at lunch and dinner, in comparison to a day without structured exercise. While the Breaks $+P A$ condition of the present study did include a total of 40 minutes of structured exercise at $60 \%$ of $\mathrm{VO}_{2}$ peak, it may be that this intensity was insufficient to influence 
subsequent food intake. It is also possible that the acute influence of exercise on caloric intake in this age group may be different among healthy weight compared to overweight/obese populations, ${ }^{(32)}$ although the present study was not sufficiently powered to examine such body weight interactions. Future studies should also investigate variations in the magnitude and direction of behavioural compensation (or lack thereof) following prolonged sitting, as exerciseinduced variations in energy expenditure and body weight have been shown to vary considerably among adults. ${ }^{(33)}$

The current findings also suggest that physical activity levels are not acutely regulated by an internal "activitystat" ${ }^{\prime(9,10)}$, as we observed no difference in physical activity or sedentary behaviour levels in the 24-hour period following each experimental condition. Instead, these results support the recent findings of Goodman and colleagues ${ }^{(34)}$ who found no evidence that a bout of physical activity during one portion of the day was compensated for with reduced physical activity later in the day among a cohort of British children. These results are in contrast to those of Thivel and colleagues ${ }^{(12)}$, who reported that an imposed bout of high- and low-intensity physical activity did not significantly increase 24-hour energy expenditure above that observed during an inactive day among obese teenagers. However, it should be noted that Thivel and colleagues assessed energy expenditure by placing participants in calorimetric chambers, which is likely to have substantially reduced their opportunities for spontaneous physical activity outside of their bouts of structured exercise. In contrast, following the in-lab portion of each condition, the present study examined physical activity levels in free-living conditions, which may help to explain these discrepant findings.

Taken together, the above findings suggest that acute sedentary behaviour may contribute to a positive energy balance due to its low level of energy expenditure, and by failing to produce a compensatory reduction in energy intake or increase in energy expenditure subsequent to the behaviour. This effect is likely to be exacerbated through the increased caloric intake that is associated with many common sedentary behaviours such as TV viewing and video game playing ${ }^{(2,4,27)}$. However, the current findings also suggest that the introduction of periodic bouts of light- and moderate-intensity physical activity throughout the day may increase energy expenditure without resulting in compensatory changes in energy intake or spontaneous physical activity. It is worth noting that physical activity intensity has been negatively associated with adiposity in the pediatric age group, and therefore the impact of breaks of vigorous intensity on energy balance are worthy of future study ${ }^{(35)}$. The current results suggest that activity breaks of at 
least light- or moderate intensity spread throughout the day may be a simple way to promote or maintain energy balance in the current sedentary and obesogenic environment ${ }^{(36)}$.

The present study has several strengths and limitations which warrant mention. The study employed a rigorous randomized crossover design, which strictly controlled participants' energy intake, sedentary behaviour and physical activity across the 3 study conditions. However, energy intake was measured only once at the end of each in-lab session, and physical activity and sedentary behaviour were only assessed in the 24-hour period immediately following each lab session. It is therefore unclear whether similar results would be seen in response to chronic exposure to prolonged sedentary behaviour. The present findings are also limited by the small sample size, and therefore the possibility of a Type 2 error cannot be ruled out. It is also worth noting that participants were required to eat standardized meals at both breakfast and lunch, which may have been different from the amount or type of food they would consume on a normal day (habitual diet was not assessed in the current study). Similarly, the buffet meal in the present study took place at 16:00, which is earlier than the typical evening meal in North America. Further, participants in the present study were healthy, and more physically active at baseline than the general Canadian population ${ }^{(13)}$. Thus these results may not generalize to physically inactive, obese or diseased participants, or to other age groups. Physical activity and sedentary behaviour levels in the present study were assessed via accelerometers, which is not able to accurately measure all forms of activity (e.g. swimming, cycling). However, the use of accelerometry allowed for the assessment of sedentary behaviour and physical activity in free-living conditions, increasing the ecological validity of these findings. Finally, the buffet in the present study included palatable items such as pizza and potato chips, which may have itself influenced $a d$ libitum intake or reduced differences across conditions ${ }^{(6)}$.

In conclusion, we found no evidence that children and youth compensate for an imposed bout of prolonged sitting, with or without breaks and structured physical activity, by decreasing their subsequent energy intake and/or increasing their physical activity levels. These findings suggest that a sedentary day may lead to a positive energy balance through reduced energy expenditure without compensatory reductions in energy intake or subsequent increases in physical activity energy expenditure. They also suggest that the introduction of light- or moderate-intensity activity breaks throughout an otherwise sedentary day may help to increase energy expenditure with no compensatory increase in food intake, thus promoting energy balance in the pediatric age 
group. Future studies with larger sample sizes are needed to further investigate the impact of prolonged sitting on energy balance in the pediatric population. 


\section{References}

1. Sedentary Behaviour Research Network. (2012) Letter to the Editor: Standardized use of the terms "sedentary" and "sedentary behaviours". Appl Physiol Nutr Metab 37, 540-542. doi: 10.1139/h2012-024

2. Chaput JP, Visby T, Nyby S et al. (2011) Video game playing increases food intake in adolescents: a randomized crossover study. Am J Clin Nutr 93, 1196-1203. doi: 10.3945/ajen.110.008680

3. Tremblay M, LeBlanc A, Kho M et al. (2011) Systematic review of sedentary behaviour and health indicators in school-aged children and youth. Int J Behav Nutr Phys Act 8, 98. doi: $10.1186 / 1479-5868-8-98$

4. Harris JL, Bargh JA, Brownell KD. (2009) Priming effects of television food advertising on eating behaviour. Health Psychol 28, 404-413. doi: 10.1037/a0014399

5. Burke V, Beilin LJ, Simmer K et al. (2004) Predictors of body mass index and associations with cardiovascular risk factors in Australian children: a prospective cohort study. Int J Obes 29(1), 15-23.

6. Thivel D, Aucouturier J, Doucet E et al. (2013) Daily energy balance in children and adolescents. Does energy expenditure predict subsequent energy intake? Appetite 60, 5864. doi: 10.1016/j.appet.2012.09.022

7. Saunders TJ. (2011) Potential Contributors to the Canadian Pediatric Obesity Epidemic. ISRN Pediatr 917684. doi: 10.5402/2011/917684

8. Saunders TJ, Chaput JP (2012) Is obesity prevention as simple as turning off the television and having a nap? Br J Nutr 108, 946-7. doi: 10.1017/S0007114512002644

9. Rowland TW. (1998) The biological basis of physical activity. Med Sci Sports Exerc 30, 392-399.

10. Frémeaux AE, Mallam KM, Metcalf BS et al. (2011) The impact of school-time activity on total physical activity: the activitystat hypothesis (EarlyBird 46). Int J Obes 35, $1277-$ 1283. doi: $10.1038 / \mathrm{ijo} .2011 .52$

11. Wilkin TJ, Mallam KM, Metcalf BS et al. (2006) Variation in physical activity lies with the child, not his environment: evidence for an "activitystat"in young children (EarlyBird 16). Int J Obes 30, 1050-5.

12. Thivel D, Isacco L, Montaurier C et al. (2012) The 24-h energy intake of obese adolescents is spontaneously reduced after intensive exercise: a randomized controlled trial in calorimetric chambers. PLoS ONE 7, e29840. doi: 10.1371/journal.pone.0029840 
13. Colley R, Garriguet D, Janssen I et al. (2011) Physical activity of Canadian children and youth: accelerometer results from the 2007 to 2009 Canadian Health Measures Survey. Health Rep 22(1),15-24.

14. Pate RR, Mitchell JA, Byun W et al. (2011) Sedentary behaviour in youth. Br J Sports Med 45, 906-913. doi: 10.1136/bjsports-2011-090192

15. Leatherdale ST, Ahmed R. (2011) Screen-based sedentary behaviours among a nationally representative sample of youth: are Canadian kids couch potatoes? Chronic Dis Inj Can 31, 141-146.

16. Saunders TJ, Chaput JP, Goldfield GS et al. (2013). Prolonged sitting and markers of cardiometabolic disease risk in children and youth: A randomized crossover study. Metabolism, In press.

17. Cole TJ, Bellizzi, MC, Flegal KM, et al. (2000). Establishing a standard definition for child overweight and obesity worldwide: international survey. BMJ 320, 1240-1243.

18. Taylor SJ, Whincup PH, Hindmarsh PC et al. (2001) Performance of a new pubertal selfassessment questionnaire: a preliminary study. Paediatr Perinat Epidemiol 15, 88-94.

19. Dubowy K-O, Baden W, Bernitzki S et al. (2008) A practical and transferable new protocol for treadmill testing of children and adults. Cardiol Young 18, 615-623. doi: $10.1017 / \mathrm{S} 1047951108003181$

20. Puyau MR, Adolph AL, Vohra FA et al. (2004) Prediction of activity energy expenditure using accelerometers in children. Med Sci Sports Exerc 36, 1625-1631.

21. World Health Organization (2002) The World Health Report 2002: reducing risks, promoting healthy life. Geneva: WHO.

22. Shomaker LB, Tanofsky-Kraff M, Zocca JM et al. (2010) Eating in the absence of hunger in adolescents: intake after a large-array meal compared with that after a standardized meal. Am J Clin Nutr 92, 697-703. doi: 10.3945/ajen.2010.29812

23. Hill AJ, Blundell JE. (1986) Macronutrients and satiety: the effects of a high-protein or high-carbohydrate meal on subjective motivation to eat and food preferences. Nutr Behav (USA) 3, 133-144.

24. Arvaniti K, Richard D, Tremblay A. (2000) Reproducibility of energy and macronutrient intake and related substrate oxidation rates in a buffet-type meal. Br J Nutr 83, 489-495.

25. Rumbold PLS, Gibson A, Allsop S et al. (2011) Energy intake and appetite following netball exercise over 5 days in trained 13-15 year old females. Appetite 56, 621-8. doi: 10.1016/j.appet.2011.02.014 
26. McNeil J, Riou MÈ, Razmjou S, et al. (2012) Reproducibility of a food menu to measure energy and macronutrient intakes in a laboratory and under real-life conditions. Br J Nutr 108, 1316-1324.

27. Chaput JP, Tremblay A. (2007) Acute effects of knowledge-based work on feeding behaviour and energy intake. Physiol Behav 90, 66-72.

28. Epstein LH, Roemmich JN, Paluch RA et al. (2005) Influence of changes in sedentary behavior on energy and macronutrient intake in youth. Am J Clin Nutr 81, 361-366. http://ajcn.nutrition.org/content/81/2/361.full.

29. Epstein LH, Roemmich JN, Robinson JL et al. (2008) A Randomized Trial of the Effects of Reducing Television Viewing and Computer Use on Body Mass Index in Young Children. Arch Pediatr Adolesc Med 162, 239-245.

30. Epstein LH, Roemmich JN, Paluch RA et al (2005) Physical activity as a substitute for sedentary behavior in youth. Ann Behav Med 29,200-209.

31. Thivel D, Blundell JE, Duché P et al. (2012) Acute exercise and subsequent nutritional adaptations: what about obese youths? Sports Med 42, 607-13. doi: 10.2165/11632460000000000-00000

32. Nemet D, Arieli R, Meckel Y et al. (2010) Immediate post-exercise energy intake and macronutrient preferences in normal weight and overweight pre-pubertal children. Int $J$ Pediatr Obes 5, 221-9. doi: 10.3109/17477160903311538

33. King NA, Caudwell P, Hopkins M et al (2007) Metabolic and behavioral compensatory responses to exercise interventions: barriers to weight loss. Obesity 15, 1373-1383.

34. Goodman A, Mackett RL, Paskins J. (2011) Activity compensation and activity synergy in British 8-13 year olds. Prev Med 53, 293-298. doi: 10.1016/j.ypmed.2011.07.019.

35. Leblanc AG Janssen I. (2010) Systematic review of the health benefits of physical activity and fitness in school-aged children and youth. Int J Behav Nutr Phys Act 7, 40. doi:10.1186/1479-5868-7-40.

36. Chaput JP, Saunders TJ. (2012) Bioenergetics of Obesity: Is Fat Gain a Problem or a Solution? Bioenergetics: Open Access 1, e101. doi:10.4172/beg.1000e101 


\section{Tables}

Table 6.1 Characteristics of study participants at baseline.

\begin{tabular}{|c|c|c|c|c|c|}
\hline & \multicolumn{2}{|c|}{ Male $(n=12)$} & \multicolumn{2}{|c|}{ Female $(n=8)$} & \multirow[b]{2}{*}{$p$ value } \\
\hline & Mean & SD & Mean & SD & \\
\hline Age (years) & 12.8 & $(1.0)$ & 11.3 & $(0.7)$ & $<0.01$ \\
\hline $\operatorname{BMI}\left(\mathrm{kg} / \mathrm{m}^{2}\right)$ & 19.4 & $(5.0)$ & 17.4 & (2.9) & 0.31 \\
\hline Proportion Overweight/Obese & $2 / 12$ & & $1 / 8$ & & 0.80 \\
\hline Waist Circumference (cm) & 68.7 & $(16.5)$ & 59.8 & $(5.7)$ & 0.16 \\
\hline Tanner Stage & 2.0 & $(1.0)$ & 1.5 & $(0.8)$ & 0.27 \\
\hline Sedentary Behaviour (min/day) & 536.4 & $(47.2)$ & 461.6 & $(66.0)$ & $<0.01$ \\
\hline LPA (min/day) & 209.6 & $(45.6)$ & 256.8 & $(33.8)$ & 0.02 \\
\hline MVPA (min/day) & 64.0 & $(28.8)$ & 59.5 & $(23.8)$ & 0.72 \\
\hline
\end{tabular}

Date are presented as mean (SD).

BMI: Body mass index; LPA: light physical activity; MVPA: moderate-and-vigorous physical activity.

Baseline differences between male and female participants were assessed by independent samples $\mathrm{t}$ test (continuous variables) and chi square test (proportions). 
Table 6.2 Measures of sedentary behaviour, physical activity, hunger and caloric intake during time spent in lab engaging in prolonged sitting, with and without breaks and structured physical activity $(n=20)$.

\begin{tabular}{|c|c|c|c|c|c|c|c|}
\hline & \multicolumn{2}{|c|}{ Sedentary } & \multicolumn{2}{|c|}{ Breaks } & \multicolumn{2}{|c|}{ Breaks+PA } & \multirow[b]{2}{*}{$p$ for trend } \\
\hline & Mean & SD & Mean & SD & Mean & SD & \\
\hline Sedentary Behaviour (min) & 498.9 & $(19.2)^{\mathrm{a}}$ & 444.3 & $(19.2)^{b}$ & 416.0 & $(19.6)^{\mathrm{c}}$ & $<0.01$ \\
\hline Light Physical Activity (min) & 12.3 & $(19.8)^{\mathrm{a}}$ & 58.6 & $(19.8)^{b}$ & 55.4 & $(20.3)^{\mathrm{b}}$ & $<0.01$ \\
\hline Moderate Physical Activity (min) & 2.3 & $(14.5)^{\mathrm{a}}$ & 10.5 & $(14.5)^{\mathrm{a}}$ & 40.9 & $(14.9)^{\mathrm{b}}$ & $<0.01$ \\
\hline Vigorous Physical Activity (min) & 0.1 & (2.3) & $<0.1$ & (2.3) & 1.3 & (2.4) & 0.18 \\
\hline Steps (steps) & 687 & $(1979)^{\mathrm{a}}$ & 4482 & $(1979)^{\mathrm{b}}$ & 8658 & $(2042)^{c}$ & $<0.01$ \\
\hline Pre-Buffet Prospective Food Consumption (mm) & 56 & $(20)$ & 66 & $(20)$ & 61 & (19) & 0.07 \\
\hline Pre-Buffet Hunger (mm) & 55 & (19) & 63 & (19) & 56 & (18) & 0.16 \\
\hline Post-Buffet Prospective Food Consumption (mm) & 13 & $(15)$ & 14 & $(15)$ & 7 & (14) & 0.18 \\
\hline Post-Buffet Hunger (mm) & 9 & $(11)$ & 8 & $(11)$ & 6 & (10) & 0.25 \\
\hline Food intake in the buffet $(\mathrm{g})$ & 782 & $(254)$ & 839 & $(254)$ & 767 & $(254)$ & 0.37 \\
\hline Calories from carbohydrates $(\%)$ & 56 & (9) & 57 & (9) & 55 & (9) & 0.62 \\
\hline Calories from fat (\%) & 33 & (9) & 32 & (9) & 36 & (9) & 0.32 \\
\hline Calories from protein $(\%)$ & 10 & (4) & 11 & (4) & 10 & (4) & 0.44 \\
\hline
\end{tabular}

Sedentary: a day of uninterrupted sitting.

Breaks: a day of sitting interrupted with a 2-minute light-intensity walk break every 20 minutes.

Breaks + Physical Activity: a day of sitting interrupted with a 2-minute light-intensity walk break every 20 minutes as well as 40 minutes of moderate-intensity physical activity.

Significance was assessed by a linear mixed-model with effects for condition, age, sex, Tanner stage, BMI and baseline physical activity and sedentary behaviour. Columns with different superscript letters are significantly different at $p<0.05$ level with Bonferroni correction. 
Table 6.3 Sedentary behaviour and physical activity levels in the 24-hours immediately following prolonged sitting with or without breaks and structured physical activity $(\mathbf{n}=\mathbf{2 0})$.

\begin{tabular}{ccccccccc}
\hline & \multicolumn{3}{c}{ Sedentary } & \multicolumn{2}{c}{ Breaks } & \multicolumn{2}{c}{ Breaks+PA } \\
\cline { 2 - 7 } & Mean & SD & Mean & SD & Mean & SD & $p$ for trend \\
\hline Wear time (min) & 700.0 & $(235.0)$ & 728.7 & $(240.9)$ & 701.1 & $(247.0)$ & 0.90 \\
Sedentary Behaviour (min) & 518.5 & $(73.0)$ & 514.4 & $(73.3)$ & 501.5 & $(74.8)$ & 0.67 \\
Sedentary Behaviour (\% wear time) & 73.6 & $(10.1)$ & 71.1 & $(10.2)$ & 70.9 & $(10.4)$ & 0.60 \\
Light Physical Activity (min) & 139.5 & $(46.2)$ & 140.8 & $(46.4)$ & 152.5 & $(47.3)$ & 0.53 \\
Light Physical Activity (\% wear time) & 19.6 & $(6.1)$ & 20.2 & $(6.1)$ & 21.3 & $(6.2)$ & 0.60 \\
Moderate Physical Activity (min) & 48.1 & $(30.8)$ & 48.0 & $(30.9)$ & 52.7 & $(31.7)$ & 0.85 \\
Moderate Physical Activity (\% wear time) & 6.4 & $(4.8)$ & 7.5 & $(4.8)$ & 7.4 & $(5.0)$ & 0.73 \\
Vigorous Physical Activity (min) & 4.2 & $(10.1)$ & 6.9 & $(10.2)$ & 3.7 & $(10.4)$ & 0.54 \\
Vigorous Physical Activity (\% wear time) & 0.5 & $(1.5)$ & 1.2 & $(1.5)$ & 0.5 & $(1.6)$ & 0.26 \\
Steps (steps) & 10596 & $(5414)$ & 11172 & $(5439)$ & 10485 & $(5561)$ & 0.89 \\
\hline
\end{tabular}

Sedentary: a day of uninterrupted sitting.

Breaks: a day of sitting interrupted with a 2-minute light-intensity walk break every 20 minutes.

Breaks + Physical Activity: a day of sitting interrupted with a 2-minute light-intensity walk break every 20 minutes as well as 40 minutes of moderate-intensity physical activity.

Significance was assessed by a linear mixed-model with effects for condition, accelerometer wear time, age, sex, Tanner stage, BMI and baseline physical activity and sedentary behaviour. There were no significant differences between experimental conditions. 


\section{Chapter 7 - General Discussion}

Sedentary behaviour has been linked with increased cardiometabolic disease risk among children and youth in multiple cross-sectional and longitudinal studies(5-12,41-45). The purpose of the present thesis was to clarify the relationship between sedentary behaviour and health in the pediatric age group by addressing two key objectives:

1. To determine the cross-sectional association of sedentary time, interruptions in sedentary time, sedentary bout length, and total movement variability with markers of cardiometabolic disease risk among children and youth.

2. To examine the impact of 1-day of prolonged sedentary behaviour, with and without interruptions or structured physical activity, on markers of cardiometabolic disease risk, hunger, food intake and spontaneous physical activity levels in children and youth.

To achieve these objectives, we have performed 4 studies, using 3 datasets, and employed both cross-sectional and interventional research methodologies. Our findings, which are summarized in Table 7.1, make an important contribution to our understanding of the health impact of sedentary behaviour among children and youth. In the discussion that follows, I will examine our results in the context of my two key objectives, and in relation to the published literature. 
Table 7.1 Summary of thesis key findings and contributions to the literature.

\begin{tabular}{|l|l|}
\hline Study 1 & $\begin{array}{l}\text { Short bouts of sedentary behaviour, breaks in sedentary time, and screen-based sedentary } \\
\text { behaviours are independently associated with cardiometabolic disease risk among } \\
\text { children with a family history of obesity. }\end{array}$ \\
\hline Study 2 & $\begin{array}{l}\text { Total movement variability is independently associated with reduced cardiometabolic } \\
\text { disease risk among a representative sample of American children and youth. }\end{array}$ \\
\hline Study 3 & $\begin{array}{l}\text { Uninterrupted sitting does not result in acute changes in markers of cardiometabolic } \\
\text { disease risk among healthy children and youth aged 10-14 years. }\end{array}$ \\
\hline Study 4 & $\begin{array}{l}\text { Uninterrupted sitting does not result in compensatory changes in subsequent energy } \\
\text { intake or physical activity levels in health children and youth aged 10-14 years. }\end{array}$ \\
\hline
\end{tabular}

\subsection{Characteristics of sedentary behaviour: associations with health indicators}

The studies presented in Chapters 3 and 4 of this thesis demonstrate that breaks in sedentary time, short bouts of sedentary behaviour (e.g. those lasting 1-4 minutes), and total movement variability are associated with reduced cardiometabolic disease risk in children and youth, independent of total sedentary time and physical activity. These cross-sectional findings suggest that, all else being equal, children who have high amounts of movement variability and frequent interruptions in their sedentary time may experience reduced cardiometabolic disease risk when compared to children with less frequent interruptions in sedentary time or reduced movement variability. The findings presented in Chapters 3 and 4 also highlight the contrasting relationships of self-reported screen time and directly measured sedentary time with markers of cardiometabolic disease risk in the pediatric population. While TV viewing and computer time were independently associated with increased cardiometabolic disease risk in girls and boys respectively, daily sedentary time assessed via accelerometer was not associated with any marker of cardiometabolic disease risk in the fully adjusted model in either sex. 
To our knowledge, the studies contained in Chapters 3 and 4 are the first to report a favourable association between breaks in sedentary time, total movement variability and global cardiometabolic disease risk in the pediatric population. These studies add to a growing body of research in adults which suggests that prolonged bouts of uninterrupted sedentary time are associated with increased health risk, while the opposite is seen for breaks in sedentary time $(13,14,25,26,46-48)$. For example, breaks in sedentary time have been reported to be independently and beneficially associated with multiple markers of cardiometabolic disease risk in adults $(13,14)$. However, recent studies in pediatric populations have failed to detect any association between breaks in sedentary time and markers of cardiometabolic disease risk $(15,16)$, with the exception of waist circumference (in boys only) in one study (15). As noted in Chapter 3, the association between breaks in sedentary time and cardiometabolic disease risk in this thesis was examined in children with a family history of obesity, while previous studies on this topic have focused primarily on nationally representative samples of Canadian and American youth $(15,16)$. It is possible that this difference in sample populations may help to explain why the findings reported in Chapter 3 diverge from previous investigations in the pediatric population.

While the associations between characteristics of sedentary time and cardiometabolic disease risk presented in Chapter 3 were examined using a cohort of children with a family history of obesity, the role of total movement variability was investigated using a representative sample of American children and youth via the National Health and Nutrition Examination Survey. The independent associations observed between movement variability and clustered cardiometabolic disease risk in Chapter 4 are 
especially noteworthy, given that previous research using this same dataset has failed to detect any association between characteristics of sedentary time and markers of cardiometabolic disease risk (16). When combined with the findings in Chapter 3 and elsewhere in the published literature $(1,9,13,14,25,26)$, a picture begins to emerge which suggests that an "ideal" movement profile may be more complicated than simply meeting national physical activity guidelines.

Table 7.2 Healthy and deleterious movement profiles for children and youth.

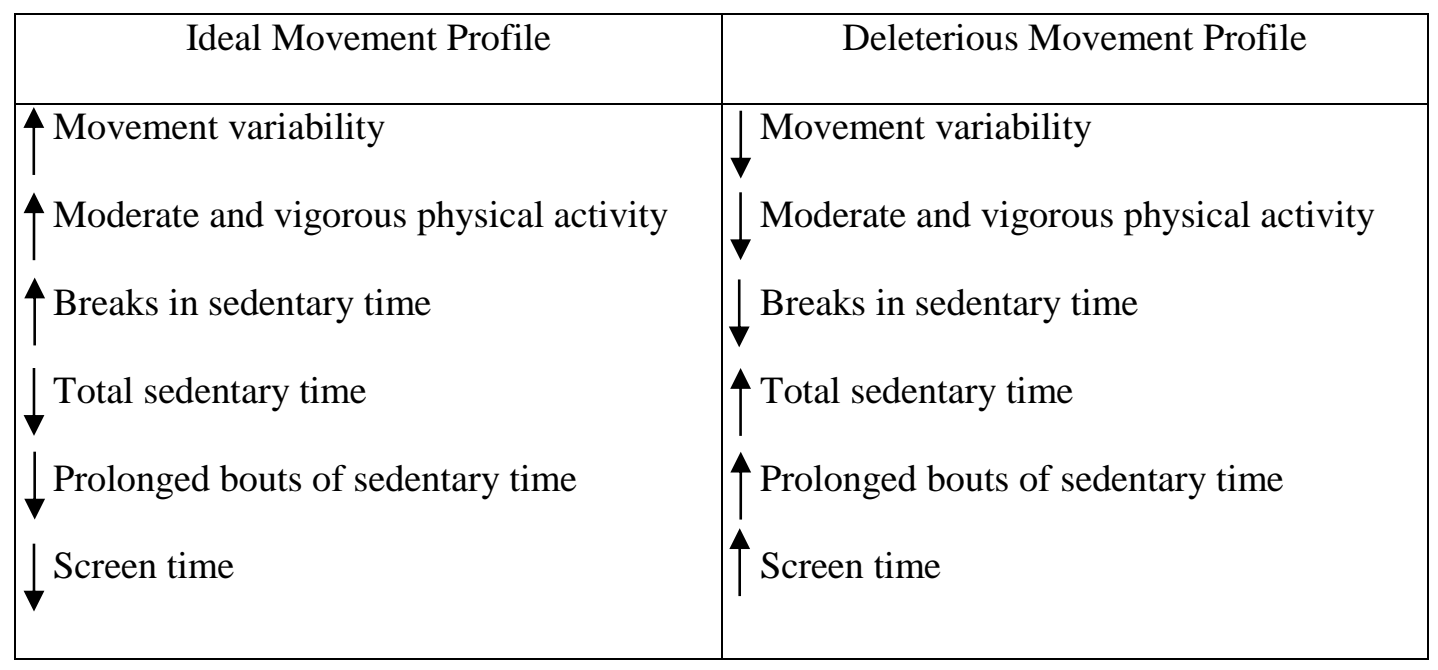

As outlined in Table 7.2 and Figure 7.1, the ideal movement profile for children and youth appears to be one which includes large amounts of physical activity, relatively little uninterrupted sitting or screen time, and frequent changes in movement intensity. However, at present the clinical relevance of breaks in sedentary time and increased movement intensity remain largely unclear in the pediatric age group. If future studies support the notion that increasing movement variability or breaks in sedentary time are associated with reduced health risk in the pediatric population, it could lead to the development of novel interventions with increased efficacy and/or effectiveness for 
treating or preventing chronic disease risk among children and youth. For example, data from the studies that make up this thesis suggest that for a given level of activity and sedentary time, interventions that produce greater increases in total movement variability and/or more frequent breaks in sedentary time could result in greater health benefits among children and youth. Both epidemiological and lab-based studies should examine the health impact of such changes in movement patterns, in order to determine whether they result in clinically significant improvements in cardiometabolic health. If intervention studies demonstrate that such changes are efficacious in improving cardiometabolic health in at-risk youth, this could lead to the development of interventions that are more practical for non-exercisers than programs which focus exclusively on structured exercise.

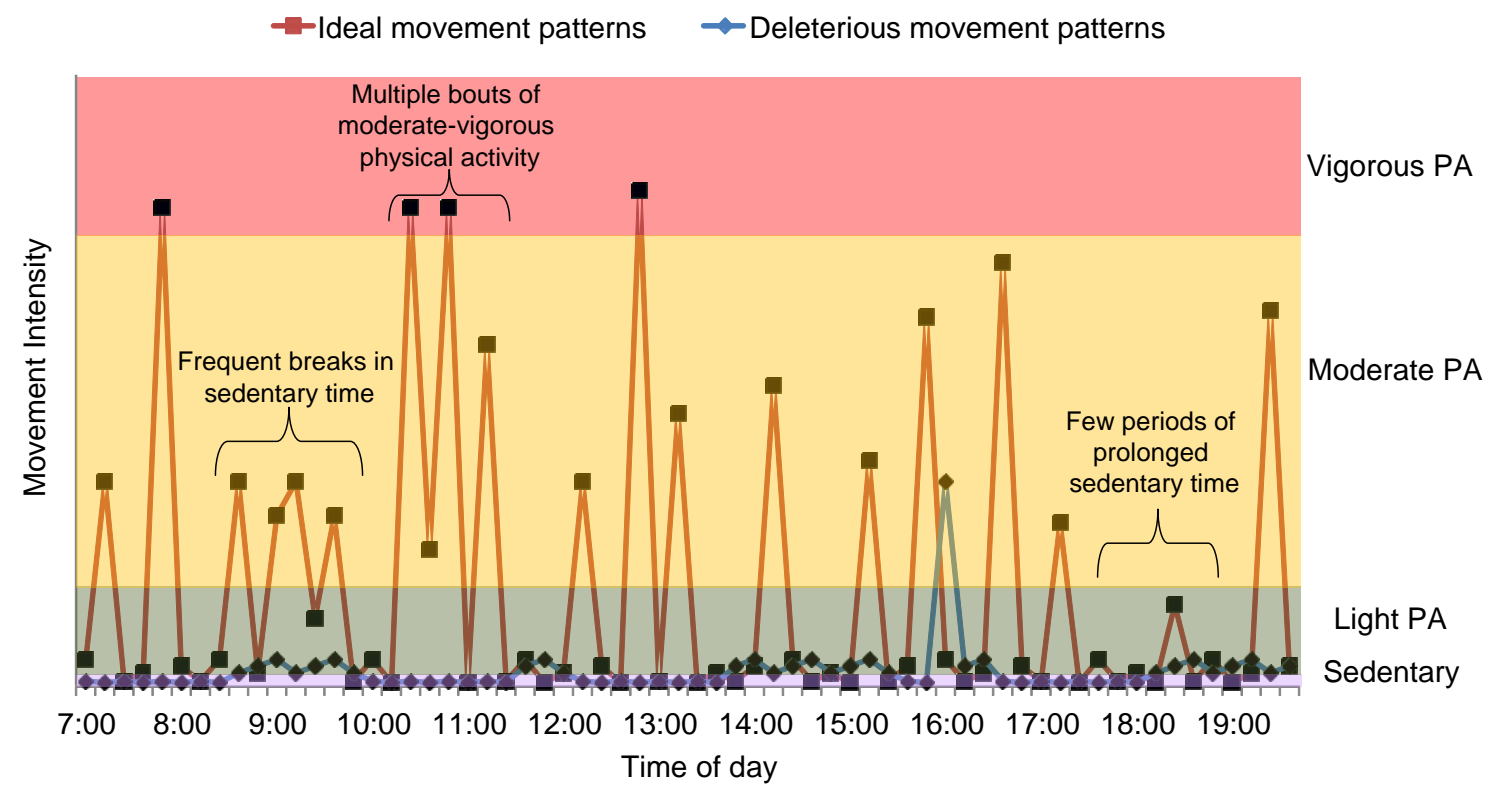

Figure 7.1 Examples of healthy and deleterious movement patterns.

In addition to any potential direct health impact of movement variability, the findings of Chapter 4 also suggest that this new metric may serve as a simple means of assessing the 
quality of individual's overall movement patterns. For example, Chapter 4 found that total movement variability is associated with a constellation of desirable movement behaviours, including high levels of physical activity, low levels of sedentary time, and frequent interruptions in sedentary time. When compared to individuals with low levels of movement variability, individuals with high movement variability are likely to accumulate higher amounts of MVPA, and lower amounts of sedentary behavior. Further, at any combination of total sedentary time and MVPA, individuals with high levels of movement variability are also likely to have more frequent breaks in sedentary time and/or higher average physical activity intensity. Rather than assessing each of these movement behaviours in isolation, movement variability could therefore be used as a global measure of a "healthy" movement profile. While further research is clearly necessary before such applications of movement variability can be made in a clinical setting, these examples nonetheless highlight the potential value of this novel movement characteristic.

\subsection{The impact of prolonged sedentary behaviour on markers of cardiometabolic disease risk, energy intake, and physical activity in children and youth}

Chapters 5 and 6 examined the metabolic and behavioural impact of prolonged sitting among a group of healthy children aged $10-14$ years. These studies failed to detect any significant impact of prolonged sitting on markers of cardiometabolic disease risk in the pediatric age group. Further, they also demonstrated that children did not compensate for an acute bout of prolonged sitting by modifying their subsequent levels of physical activity, sedentary behaviour, or food intake. As with the cross-sectional association between breaks in sedentary time and markers of cardiometabolic disease risk (which has been observed more consistently in adult populations than in children and youth), the 
results from Chapters 5 and 6 suggest that the acute impact of prolonged sitting may also differ according to sample population. For example, interventions which impose prolonged sitting have resulted in acute reductions in insulin sensitivity and glucose tolerance in multiple studies among adults $(25,46,49)$. We did not observe any such change among healthy children and youth in the present thesis, despite sitting for a comparable amount of time. It is possible that changes in markers of cardiometabolic disease risk may have been observed had the present intervention focused on a different participant population, such as children and youth with elevated risk of chronic disease, or those with a family history of obesity (similar to the participants in Chapter 3). As discussed below, this is an important area for future research in the pediatric age group.

The data presented in Chapters 5 and 6 also help to explain the disparate associations of objectively measured sedentary time and screen time observed in Chapter 3 and in the field more generally. Numerous studies $(9,11,12,16,50)$ have found that screen-based sedentary behaviours are associated with markers of cardiometabolic disease risk in children and youth. However, although some studies have reported a significant association between objectively measured sedentary time and markers of cardiometabolic disease risk in this population $(5,10,44,51-53)$, many others (including Chapter 3 of this thesis) have failed to detect such a relationship $(15,16,50,54-59)$. In Chapter 6 of this thesis we report that prolonged sitting did not result in significant changes in markers of cardiometabolic disease risk or subsequent food intake or physical activity in children and youth. In contrast, both observational and experimental evidence suggests that screen-based sedentary behaviours result in increased ad libitum food intake in this population $(29,34,60-62)$ Thus, while prolonged sitting and screen-based sedentary behaviours may result in less movement and promote incidental snacking, the resulting 
positive energy balance appears to be more related to screen-based sedentary behaviours. This is likely one reason why cardiometabolic disease risk is more consistently associated with screen-based sedentary behaviours than with total sedentary time in the pediatric population, as observed in Chapter 3.

\subsubsection{Does sitting per se have a negative impact on pediatric health?}

Given that neither objectively measured total sedentary time nor prolonged sitting were associated with increased cardiometabolic disease risk in the present thesis, some may ask whether it is worth promoting reductions in sedentary time in the pediatric age group. However, the balance of evidence continues to suggest that high levels of total sedentary time should be avoided by all age groups, including children and youth. Though not equivocal, several cross-sectional and longitudinal studies have found significant associations between sedentary behaviour and cardiometabolic disease risk among children and youth $(5,10,44,51-53)$. As discussed previously, results from Chapter 6 suggest that even in the absence of acute changes in markers of cardiometabolic disease risk, prolonged sitting is still likely to lead to positive energy balance, and potential weight gain. Further, as reported in Chapter 3, certain characteristics of sedentary behaviour (e.g. breaks and/or bout length) may be associated with risk factors for chronic disease in this age group, even if total sedentary time is not. It is also possible that longer (e.g. $>8$ hours) exposure to uninterrupted sedentary behaviours, or repeated exposures over a period of several days, may result in detectable adverse changes in cardiometabolic disease risk biomarkers.

Even if the potential immediate health impact of total sedentary time in the pediatric age group is disregarded, excessive sitting in childhood may still set children on a path for 
poor health in later life. Both total sedentary time and the number of prolonged bouts of sedentary behaviour increase with age $(56,63,64)$; therefore participants with high levels of sedentary behaviour in childhood are likely to experience even greater levels in adulthood. This is troubling, since total (self-reported) sedentary time in adulthood has been linked with increased risk of chronic disease morbidity and mortality (65).

Similarly, while we failed to detect an acute deleterious effect of prolonged sitting in the present thesis, it has been reported to reduce insulin sensitivity and glucose tolerance in several studies among adults $(25,46,49,66)$. Further, sedentary time appears to track moderately well through childhood, such that the most sedentary individuals among a population at one time point are likely to remain so later on $(67,68)$. Therefore, while the immediate health impact of prolonged sitting in childhood may be small, it may nevertheless result in increased cardiometabolic disease risk in adulthood.

The findings of the present thesis also lend support to the wording used in Canada's Sedentary Behaviour Guidelines, which were initially released in 2011 (41). The current guidelines focus on screen-based sedentary behaviours, stating that school-aged children and youth should "limit recreational screen time to no more than 2 hours per day; lower levels are associated with additional health benefits". However, they also state that children and youth should "limit sedentary (motorized) transport, extended sitting and time spent indoors throughout the day." Given the findings presented in this thesis and elsewhere in the published literature, the current approach which focuses primarily on screen-based sedentary behaviours, but which also suggests limiting prolonged sitting more generally, seems appropriate (41). 


\subsection{Opportunities for future research}

The results of the present thesis suggest a number of potential areas for future research, several of which have been briefly alluded to previously. These studies are discussed below and grouped according to study methodology.

\subsubsection{Observational studies}

The results of the present thesis raise several questions that can be explored further using existing cross-sectional databases. Chapter 4 of this thesis is the first study to examine the impact of movement variability in any population. Therefore, replication studies are needed to examine the association between movement variability and health in other populations of children, as well as examining similar relationships in adults, and in populations that are already at elevated risk for chronic disease. Longitudinal studies that examine the relationship between movement variability and health over time could also yield interesting results, and are now feasible given a growing number of longitudinal databases that include objective measures of sedentary time at multiple time points $(13,17,44)$. These studies will help to determine whether movement variability represents a novel and distinct risk factor for chronic disease, and establish whether interventions targeting movement variability are worthy of investigation.

In addition to total movement variability, further studies are also needed to clarify the association of breaks in sedentary time and sedentary bout length with health in the pediatric age-group. Although the study presented in Chapter 3 found significant associations between breaks in sedentary time and clustered cardiometabolic disease risk in our sample of children with a family history of obesity, other studies have failed to detect such an association in samples of the general population $(15,16)$. This suggests 
patterns of sedentary behaviour may be particularly important in specific groups of children, and future studies should examine whether this is the case. As with total movement variability, longitudinal studies are likely to be useful in deciding whether interventions targeting breaks in sedentary time are warranted in pediatric or adult populations.

\subsubsection{Intervention studies}

The results presented in Chapters 5 and 6 suggest that prolonged sitting has a minimal acute impact on metabolic health, energy intake and physical activity in healthy children and youth between the ages of 10 and 14 years. However, it is unclear whether such findings would be observed in older youth, or in those with increased markers of cardiometabolic disease risk. Studies have suggested that prolonged sitting and other forms of sedentary behaviour result in acute increases in markers of cardiometabolic disease risk in healthy young adults $(46,49,69-72)$. It is therefore possible that similar findings may be observed in older teens as well.

It is also possible that the majority of participants in Studies 5 and 6 were in such robust health that 1 day of prolonged sitting was insufficient to result in changes in markers of cardiometabolic disease risk. Acute changes in markers of cardiometabolic disease risk following prolonged sitting may be more consistent, and more clinically relevant, in children and youth with elevated cardiometabolic disease risk at study entry. It is worth noting that individuals living with chronic diseases such as the metabolic syndrome, cardiovascular disease and type 2 diabetes have been largely overlooked by intervention studies targeting sedentary behaviour in both adults and children (26). This omission is 
especially glaring given that these populations may be most likely to gain from lifestyle interventions that promote reductions in sedentary behaviour.

When compared to traditional lifestyle interventions that focus on structured physical activity, reductions in sedentary time (or increasing the frequency of breaks in sedentary time) are likely to be easier to implement and may promote better adherence, given that they require few skills, resources or physical effort. Although several studies have examined the impact of reduced screen-based sedentary behaviours on body weight in children and youth $(9,73,74)$, to date no studies have examined the metabolic impact of reducing total sedentary time in any pediatric population. Similarly, while a small number of studies have examined the impact of reducing total sedentary time in adult populations, most interventions to date have focused on feasibility rather than health outcomes (75-82). As a result, the efficacy and effectiveness of reducing total sedentary time remains unclear in both pediatric and adult populations. Studies examining the cardiometabolic impact of reducing total sedentary time, or specific sedentary behaviours, are therefore greatly needed moving forward.

It is also necessary to further investigate whether the health impact of sedentary behaviour can be modified through the adoption of other health behaviours. For example, Stephens et al. reported that the impact of prolonged sitting on insulin sensitivity was cut in half by simply reducing energy intake to match expenditure (46). Similarly, a recent intervention by Duvivier and colleagues (49) reported that an hour of daily vigorous exercise does not eliminate the cardiometabolic impact of prolonged sitting. Aside from the studies presented within the present thesis, to date no studies have examined such issues in the pediatric age group. A better understanding of the 
interaction between sedentary behaviour and other health behaviours is needed in order to tailor both clinical and public health messages targeting the prevention of chronic diseases.

Finally, interventions are needed that directly compare the health and behavioural impact of common sedentary behaviours. For example, previous studies have suggested that both video games and TV viewing increase ad libitum food intake in children and youth $(29,34,60,61)$. However, to our knowledge these behaviours have yet to be compared to each other, or with other common forms of sedentary behaviour such as reading or texting. As with projects discussed previously, these comparative studies may help target clinical and public health interventions towards those forms of sedentary behaviour which are most closely associated with deleterious health or health behaviours.

\subsection{Thesis strengths and limitations}

The current thesis has a number of strengths and weaknesses that warrant mention.The studies in this thesis focus on North American children and youth between the ages of 8 and 17 years. Thus, the findings generated from these studies may not generalize to other populations or age-groups. The first two investigations in this thesis (Chapters 3 and 4) were cross-sectional in nature, and therefore cannot make conclusions related to causality. Further, these studies examined only a brief snapshot of each individual's exposure to sedentary behaviour, and thus may not represent the impact of chronic sedentary behaviour. 
While the design of the third and fourth studies in this thesis (tightly controlled randomized crossover studies) allow for conclusions related to causality, the results may not generalize to more ecologically valid situations, or to cumulative bouts of prolonged sitting performed over a period of days or weeks. It should also be noted that participants in these studies were healthy and more active than the typical Canadian child, which may have influenced our findings towards the null. As discussed previously, future research should examine whether similar findings are observed in children and youth who are physically inactive, highly sedentary, or who have elevated markers of cardiometabolic disease risk.

Despite the abovementioned limitations, the studies that form this thesis have a number of key strengths which allow them to make an important contribution to our understanding of the health impact of sedentary behaviour in the pediatric age-group. The primary strength of this thesis is that it employed a variety of approaches ranging from large-scale nationally representative surveys to rigorously controlled lab-based intervention studies. This allowed for the examination of associations at the population level, as well as examining causal relationships and possible mechanisms in a more controlled setting. This approach provided insight into the mechanisms underlying key issues (for example, the frequent discrepancies in the strength of health-related associations for self-reported and objectively measured sedentary time in Chapter 3 of this thesis and in the literature more generally) which would not have been possible had only one methodology been applied to all studies. Finally, each study within this thesis also included the direct measurement of important markers of cardiometabolic disease risk, as well as objective measurement of physical activity and sedentary behaviour. 


\subsection{Conclusions}

The present thesis demonstrates that breaks in sedentary time, short bouts of sedentary behaviour and total movement variability are cross-sectionally associated with reduced cardiometabolic disease risk independent of physical activity and sedentary behaviour in certain populations of children and youth. Self-reported TV viewing and computer use were also strongly and independently associated with increased cardiometabolic disease risk in girls and boys, respectively. Despite these cross-sectional findings, in our intervention studies we found no acute impact of prolonged sitting, with or without breaks and structured physical activity, on markers of cardiometabolic disease risk, subsequent energy intake, or physical activity levels in healthy youth aged 10-14 years. The findings of these intervention studies show that prolonged sitting does not have an immediate impact on markers of cardiometabolic disease risk in children and youth, although it may predispose to positive energy balance. Collectively, these findings suggest that optimal levels of cardiometabolic disease risk are most likely to be seen in children who limit their time engaging in screen-based sedentary behaviours, who frequently interrupt their sedentary time, and who have high levels of variability in their movement behaviours. 


\section{Chapter 8 - Bibliography for Chapters 1 and 7}

1. Janssen I, LeBlanc AG. Systematic review of the health benefits of physical activity and fitness in school-aged children and youth. Int. J. Behav. Nutr. Phys. Act. 2010;7(1):40.

2. Andersen LB, Harro M, Sardinha LB, Froberg K, Ekelund U, Brage S, et al. Physical activity and clustered cardiovascular risk in children: a cross-sectional study (The European Youth Heart Study). The Lancet. 2006;368(9532):299-304.

3. Schmitz KH, Jacobs DR, Hong CP, Steinberger J, Moran A, Sinaiko AR. Association of physical activity with insulin sensitivity in children. Int. J. Obes. 2002;26(10):1310-6.

4. Sedentary Behaviour Research Network. Letter to the Editor: Standardized use of the terms "sedentary" and "sedentary behaviours."Appl. Physiol. Nutr. Metab. Physiol. Appliquée Nutr. Métabolisme. 2012 Jun;37(3):540-2.

5. Sardinha LB, Andersen LB, Anderssen SA, Quitério AL, Ornelas R, Froberg K, et al. Objectively Measured Time Spent Sedentary Is Associated With Insulin Resistance Independent of Overall and Central Body Fat in 9- to 10-Year-Old Portuguese Children. Diabetes Care. 2008 Mar;31(3):569 -575.

6. Danielsen Y, Júlíusson P, Nordhus I, Kleiven M, Meltzer H, Olsson S, et al. The relationship between life-style and cardio-metabolic risk indicators in children: the importance of screen time. Acta Paediatr. 2011 Feb;100(2):253-9.

7. Ekelund U, Brage S, Froberg K, Harro M, Anderssen SA, Sardinha LB, et al. TV Viewing and Physical Activity Are Independently Associated with Metabolic Risk in Children: The European Youth Heart Study. Plos Med. 2006 Dec 12;3(12):e488.

8. Mark AE, Janssen I. Relationship between screen time and metabolic syndrome in adolescents. J. Public Health. 2008;30(2):153.

9. Tremblay M, LeBlanc A, Kho M, Saunders T, Larouche R, Colley R, et al. Systematic review of sedentary behaviour and health indicators in school-aged children and youth. Int. J. Behav. Nutr. Phys. Act. 2011 Sep 21;8(1):98. 
10. Cliff DP, Okely AD, Burrows TL, Jones RA, Morgan PJ, Collins CE, et al.

Objectively measured sedentary behavior, physical activity, and plasma lipids in overweight and obese children. Obesity. 2013;21(2):382-5.

11. Goldfield GS, Kenny GP, Hadjiyannakis S, Phillips P, Alberga AS, Saunders TJ, et al. Video game playing is independently associated with blood pressure and lipids in overweight and obese adolescents. Plos One. 2011;6(11):e26643.

12. Goldfield GS, Saunders TJ, Kenny GP, Hadjiyannakis S, Phillips P, Alberga AS, et al. Screen Viewing and Diabetes Risk Factors in Overweight and Obese Adolescents. Am. J. Prev. Med. 2013 Apr;44(4):S364-S370.

13. Healy GN, Dunstan DW, Salmon J, Cerin E, Shaw JE, Zimmet PZ, et al. Breaks in sedentary time: beneficial associations with metabolic risk. Diabetes Care. 2008 Apr;31(4):661-6.

14. Healy GN, Matthews CE, Dunstan DW, Winkler EAH, Owen N. Sedentary time and cardio-metabolic biomarkers in US adults: NHANES 2003-06. Eur. Heart J. 2011 Mar;32(5):590-7.

15. Colley RC, Garriguet D, Janssen I, Saunders TJ, Carson V, Wong S, et al. The association between accelerometer-measured patterns of sedentary time and health risk in children and youth: a cross-sectional study. BMC Public Health. 2013;13(200).

16. Carson V, Janssen I. Volume, patterns, and types of sedentary behavior and cardio-metabolic health in children and adolescents: a cross-sectional study. BMC Public Health. 2011;11(1):274.

17. Kwon S, Burns TL, Levy SM, Janz KF. Breaks in sedentary time during childhood and adolescence: Iowa bone development study. Med. Sci. Sports Exerc. 2012 Jun;44(6):1075-80.

18. Oliver M, Schluter PJ, Healy GN, Tautolo E-S, Schofield G, Rush E. Breaks in Sedentary Time - Associations with Body Size in Pacific Mothers and their Children: Findings from the Pacific Islands Families Study. J. Phys. Act. Health. 2012 Dec 3;

19. Mutch WA, Eschun GM, Kowalski SE, Graham MR, Girling LG, Lefevre GR. Biologically variable ventilation prevents deterioration of gas exchange during prolonged anaesthesia. Br. J. Anaesth. 2000 Feb;84(2):197-203.

20. Taleb NN. Antifragile: Things That Gain from Disorder. Random House; 2012. 
21. West BJ. Where Medicine Went Wrong: Rediscovering the Path to Complexity (Studies of Nonlinear Phenomena in Life Science). 1st ed. World Scientific Publishing Company; 2006.

22. Mattson MP, Wan R. Beneficial effects of intermittent fasting and caloric restriction on the cardiovascular and cerebrovascular systems. J. Nutr. Biochem. 2005 Mar;16(3):129-37.

23. Brewster JF, Graham MR, Mutch WAC. Convexity, Jensen's inequality and benefits of noisy mechanical ventilation. J. R. Soc. Interface. 2005 Sep 22;2(4):393-6.

24. Stephens BR, Granados K, Zderic TW, Hamilton MT, Braun B. Effects of 1 day of inactivity on insulin action in healthy men and women: interaction with energy intake. Metabolism. 2011; 60(7):941-9.

25. Dunstan DW, Kingwell BA, Larsen R, Healy GN, Cerin E, Hamilton MT, et al. Breaking up prolonged sitting reduces postprandial glucose and insulin responses. Diabetes Care. 2012;35(5):976-83.

26. Saunders TJ, Larouche R, Colley RC, Tremblay MS. Acute Sedentary Behaviour and Markers of Cardiometabolic Risk: A Systematic Review of Intervention Studies. J. Nutr. Metab. 2012;2012:1-12.

27. Bey L. Suppression of skeletal muscle lipoprotein lipase activity during physical inactivity: a molecular reason to maintain daily low-intensity activity. J. Physiol. 2003 Aug;551(2):673-82.

28. Wiecha JL, Peterson KE, Ludwig DS, Kim J, Sobol A, Gortmaker SL. When children eat what they watch: impact of television viewing on dietary intake in youth. Arch. Pediatr. Adolesc. Med. 2006;160(4):436.

29. Halford JCG, Gillespie J, Brown V, Pontin EE, Dovey TM. Effect of television advertisements for foods on food consumption in children.Appetite. 2004 Apr;42(2):221-5.

30. Epstein LH, Paluch R, Smith JD, Sayette M. Allocation of attentional resources during habituation to food cues. Psychophysiology. 1997;34(1):59-64.

31. Chaput JP, Klingenberg L, Astrup A, Sjödin AM. Modern sedentary activities promote overconsumption of food in our current obesogenic environment. Obes. Rev. 2011; 12(5):e12-20. 
32. Thivel D, Aucouturier J, Doucet E, Saunders TJ, Chaput J-P. Daily energy balance in children and adolescents. Does energy expenditure predict subsequent energy intake? Appetite. 2013;60(1):58-64.

33. Pearson N, Biddle SJH. Sedentary behavior and dietary intake in children, adolescents, and adults.A systematic review. Am. J. Prev. Med. 2011 Aug;41(2):178-88.

34. Harris JL, Bargh JA, Brownell KD. Priming effects of television food advertising on eating behavior. Health Psychol. 2009;28(4):404.

35. Wilkin TJ, Mallam KM, Metcalf BS, Jeffery AN, Voss LD. Variation in physical activity lies with the child, not his environment: evidence for an "activitystat"in young children (EarlyBird 16). Int. J. Obes. 2006;30(7):1050-5.

36. Mallam KM, Metcalf BS, Kirkby J, Voss LD, Wilkin TJ. Contribution of timetabled physical education to total physical activity in primary school children: cross sectional study. Bmj. 2003;327(7415):592.

37. Hagströmer M, Elmberg K, M \aarild S, Sjöström M. Participation in organized weekly physical exercise in obese adolescents reduced daily physical activity. Acta Paediatr. 2009;98(2):352-4.

38. Colley R, Garriguet D, Janssen I, Craig C, Clarke J, Tremblay M. Physical activity of Canadian children and youth: accelerometer results from the 2007 to 2009 Canadian Health Measures Survey. Health Rep. 2011;22(1):15-24.

39. Nettlefold L, McKay HA, Warburton DER, McGuire KA, Bredin SSD, Naylor PJ. The challenge of low physical activity during the school day: at recess, lunch and in physical education. Br. J. Sports Med. 2011; 45(10):813-819.

40. Active Healthy Kids Canada. Are we driving our kids to unhealthy habits? [Internet].[cited 2013 May 14]. 2013. Available from: http://activehealthykids.ca/ReportCard/ReportCardOverview.aspx

41. Tremblay MS, LeBlanc AG, Janssen I, Kho ME, Hicks A, Murumets K, et al. Canadian Sedentary Behaviour Guidelines for Children and Youth. Appl. Physiol. Nutr. Metab. 2011;36(1):59-64.

42. Kaur H, Choi WS, Mayo MS, Jo Harris K. Duration of television watching is associated with increased body mass index. J. Pediatr. 2003 Oct;143(4):506-11. 
43. Mitchell JA, Pate RR, Liese AD. Changes in cardiovascular disease risk factors from age 9 to 19 and the influence of television viewing.Obes. Silver Spring Md. 2013 Feb;21(2):386-93.

44. Mitchell JA, Pate RR, Beets MW, Nader PR. Time spent in sedentary behavior and changes in childhood BMI: a longitudinal study from ages 9 to 15 years. Int. J. Obes. 2005. 2013 Jan;37(1):54-60.

45. Proctor MH, Moore LL, Gao D, Cupples LA, Bradlee ML, Hood MY, et al. Television viewing and change in body fat from preschool to early adolescence: The Framingham Children's Study. Int. J. Obes. 2003 Jun 18;27(7):827-33.

46. Stephens BR, Granados K, Zderic TW, Hamilton MT, Braun B. Effects of 1 day of inactivity on insulin action in healthy men and women: interaction with energy intake. Metabolism. 2011;60(7):941-9.

47. Cooper AR, Sebire S, Montgomery AA, Peters TJ, Sharp DJ, Jackson N, et al. Sedentary time, breaks in sedentary time and metabolic variables in people with newly diagnosed type 2 diabetes. Diabetologia. 2012 Mar 1;55(3):589-99.

48. Henson J, Yates T, Biddle SJH, Edwardson CL, Khunti K, Wilmot EG, et al. Associations of objectively measured sedentary behaviour and physical activity with markers of cardiometabolic health. Diabetologia. 2013 May;56(5):1012-20.

49. Duvivier BMFM, Schaper NC, Bremers MA, van Crombrugge G, Menheere PPCA, Kars M, et al. Minimal Intensity Physical Activity (Standing and Walking) of Longer Duration Improves Insulin Action and Plasma Lipids More than Shorter Periods of Moderate to Vigorous Exercise (Cycling) in Sedentary Subjects When Energy Expenditure Is Comparable. Plos One. 2013 Feb 13;8(2):e55542.

50. Chaput JP, Saunders TJ, Mathieu ME, Henderson M, Tremblay MS, O’Loughlin $\mathrm{J}$, et al. Combined associations between moderate to vigorous physical activity and sedentary behavior with cardiometabolic risk factors in children. Appl. Physiol. Nutr. Metab. 2013;38(5):477-83.

51. Henderson M, Gray-Donald K, Mathieu M-E, Barnett TA, Hanley JA, O'Loughlin J, et al. How Are Physical Activity, Fitness, and Sedentary Behavior Associated With Insulin Sensitivity in Children? Diab Care. 2012 Jun $1 ; 35(6): 1272-8$. 
52. Atkin AJ, Ekelund U, Møller NC, Froberg K, Sardinha LB, Andersen LB, et al. Sedentary Time in Children: Influence of Accelerometer Processing on Health Relations. Med. Sci. Sports Exerc. 2012 Dec 27;

53. Kriska A, Delahanty L, Edelstein S, Amodei N, Chadwick J, Copeland K, et al. Sedentary behavior and physical activity in youth with recent onset of type 2 diabetes. Pediatrics. 2013 Mar;131(3):e850-856.

54. Ekelund U, Luan J, Sherar LB, Esliger DW, Griew P, Cooper A. Moderate to vigorous physical activity and sedentary time and cardiometabolic risk factors in children and adolescents. Jama J. Am. Med. Assoc. 2012 Feb 15;307(7):704-12.

55. Martinez-Gomez D, Eisenmann JC, Healy GN, Gomez-Martinez S, Diaz LE, Dunstan DW, et al. Sedentary Behaviors and Emerging Cardiometabolic Biomarkers in Adolescents. J. Pediatr. 2012 Jan;160(1):104-110.e2.

56. Kwon S, Burns TL, Levy SM, Janz KF. Which Contributes More to Childhood Adiposity-High Levels of Sedentarism or Low Levels of Moderate-throughVigorous Physical Activity? The Iowa Bone Development Study. J. Pediatr. 2013 Jan 7;

57. Chaput J-P, Lambert M, Mathieu M-E, Tremblay MS, O’ Loughlin J, Tremblay A. Physical activity vs. sedentary time: independent associations with adiposity in children. Pediatr.Obes. 2012;7(3):251-8.

58. Steele RM, van Sluijs EMF, Cassidy A, Griffin SJ, Ekelund U. Targeting sedentary time or moderate- and vigorous-intensity activity: independent relations with adiposity in a population-based sample of 10-y-old British children. Am. J. Clin. Nutr. 2009 Nov;90(5):1185-92.

59. Martinez-Gomez D TJ. Associations between sedentary behavior and blood pressure in young children. Arch. Pediatr. Adolesc. Med. 2009 Aug 1;163(8):724-30.

60. Chaput JP, Visby T, Nyby S, Klingenberg L, Gregersen NT, Tremblay A, et al. Video game playing increases food intake in adolescents: a randomized crossover study. Am. J. Clin. Nutr. 2011;93(6):1196-203.

61. Halford JCG, Boyland EJ, Hughes G, Oliveira LP, Dovey TM. Beyond-brand effect of television (TV) food advertisements/commercials on caloric intake and food choice of 5-7-year-old children.Appetite. 2007 Jul;49(1):263-7. 
62. Wiecha JL, Peterson KE, Ludwig DS, Kim J, Sobol A, Gortmaker SL. When children eat what they watch: impact of television viewing on dietary intake in youth. Arch. Pediatr. Adolesc. Med. 2006;160(4):436.

63. Trang NHHD, Hong TK, van der Ploeg HP, Hardy LL, Kelly PJ, Dibley MJ. Longitudinal Sedentary Behavior Changes in Adolescents in Ho Chi Minh City. Am. J. Prev. Med. 2013 Mar;44(3):223-30.

64. Brodersen NH, Steptoe A, Boniface DR, Wardle J. Trends in physical activity and sedentary behaviour in adolescence: ethnic and socioeconomic differences. Br. J. Sports Med. 2007 Mar 1;41(3):140-4.

65. Wilmot EG, Edwardson CL, Achana FA, Davies MJ, Gorely T, Gray LJ, et al. Sedentary time in adults and the association with diabetes, cardiovascular disease and death: systematic review and meta-analysis. Diabetologia. 2012;1-11.

66. Nygaard H, Tomten SE, Høstmark AT. Slow postmeal walking reduces postprandial glycemia in middle-aged women. Appl. Physiol. Nutr. Metab. 2009 Dec;34(6):1087-92.

67. Baggett CD, Stevens J, McMurray RG, Evenson KR, Murray DM, Catellier DJ, et al. Tracking of physical activity and inactivity in middle school girls. Med. Sci. Sports Exerc. 2008 Nov;40(11):1916-22.

68. Biddle SJH, Pearson N, Ross GM, Braithwaite R. Tracking of sedentary behaviours of young people: A systematic review. Prev. Med. 2010 Nov;51(5):345-51.

69. Hamburg NM, McMackin CJ, Huang AL, Shenouda SM, Widlansky ME, Schulz E, et al. Physical inactivity rapidly induces insulin resistance and microvascular dysfunction in healthy volunteers. Arterioscler.Thromb.Vasc. Biol.

2007;27(12):2650.

70. Yaroshenko YY, Zorban YG, Kuznetsov NK, Kakurin AG, Popov VK, Yazulin VL. Changes in thyroid hormones and lipids in endurance trained volunteers during acute and rigorous bed rest conditions. Wien. Klin.Wochenschr. 1998;110:225-31.

71. Yanagibori R, Suzuki Y, Kawakubo K, Kondo K, Iwamoto T, Itakura H, et al. The effects of 20 days bed rest on serum lipids and lipoprotein concentrations in healthy young subjects. J. Gravitational Physiol. J. Int. Soc. Gravitational Physiol. 1997 Jan;4(1):S82-90. 
72. Bergouignan A, Rudwill F, Simon C, Blanc S. Physical inactivity as the culprit of metabolic inflexibility: evidence from bed-rest studies. J. Appl. Physiol.

2011;111(4):1201-10.

73. Epstein LH, Roemmich JN, Robinson JL, Paluch RA, Winiewicz DD, Fuerch JH, et al. A randomized trial of the effects of reducing television viewing and computer use on body mass index in young children. Arch. Pediatr. Adolesc. Med. 2008;162(3):239.

74. Robinson TN. Reducing children's television viewing to prevent obesity: A randomized controlled trial. JAMA. 1999 Oct 27;282(16):1561-7.

75. Healy GN, Eakin EG, LaMontagne AD, Owen N, Winkler EAH, Wiesner G, et al. Reducing sitting time in office workers: Short-term efficacy of a multicomponent intervention. Prev. Med. 2013 Jul;57(1):43-8.

76. John D, Thompson DL, Raynor H, Bielak K, Rider B, Bassett DR. Treadmill workstations: a worksite physical activity intervention in overweight and obese office workers. J. Phys. Act. Health. 2011 Nov;8(8):1034-43.

77. John D, Bassett D, Thompson D, Fairbrother J, Baldwin D. Effect of using a treadmill workstation on performance of simulated office work tasks. J. Phys. Act. Health. 2009 Sep;6(5):617-24.

78. Thompson WG, Levine JA. Productivity of transcriptionists using a treadmill desk. Work J. Prev. Assess. Rehabil. 2011 Jan 1;40(4):473-7.

79. Koepp GA, Manohar CU, McCrady-Spitzer SK, Ben-Ner A, Hamann DJ, Runge CF, et al. Treadmill desks: A 1-year prospective trial. Obesity. 2013;21(4):70511.

80. Straker L, Abbott RA, Heiden M, Mathiassen SE, Toomingas A. Sit-stand desks in call centres: associations of use and ergonomics awareness with sedentary behavior. Appl. Ergon. 2013 Jul;44(4):517-22.

81. Gilson ND, Suppini A, Ryde GC, Brown HE, Brown WJ. Does the use of standing "hot" desks change sedentary work time in an open plan office? Prev. Med. 2012 Jan;54(1):65-7.

82. Grunseit AC, Chau JY-Y, van der Ploeg HP, Bauman A. "Thinking on your feet": A qualitative evaluation of sit-stand desks in an Australian workplace. BMC Public Health. 2013 Apr 18;13(1):365. 
Chapter 9 - Appendices 


\section{Appendix A: Ethics approval notices for thesis research projects}

\subsection{Study 1}

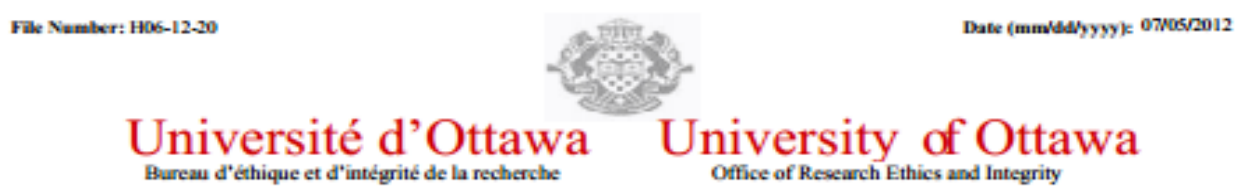

Ethics Approval Notice

Health Sciences and Science REB

Principal Investigator / Supervisor / Co-investigator(s) / Student(s)

$\begin{array}{llll}\text { First Name } & \text { Last Name } & \text { Affiliation } & \text { Role } \\ \text { Mark } & \text { Tremblay } & \text { Health Sciences/Human Kinetics } & \text { Supervisor } \\ \text { Travis } & \text { Saunders } & \text { Health Sciences/Others } & \text { Student Researcher } \\ \text { Angelo } & \text { Tremblay } & \text { Others/Others } & \text { Other Collaborator }\end{array}$

File Number: Ho6-12-20

Type of Project: Secondary use of data

Title: The Health Impact of Sedentary Behaviour in Children and Youth

Approval Date (mm/dd/yyyy)

Expiry Date (mm/dd/yyyy)

Approval Type

$07 / 05 / 2012$

$07 / 04 / 2013$

la

(la: Approval, lb: Approval for initial stage only)

Special Conditions / Comments:

N/A

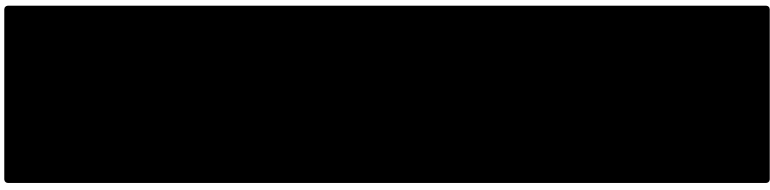




\section{Université d'Ottawa University of Ottawa}

This is to confirm that the University of Ottawa Research Ethics Board identified above, which operates in accordance with the Tri-Council Policy Statement and other applicable laws and regulations in Ontario, has examined and approved the application for ethical approval for the above named research project as of the Ethics Approval Date indicated for the period above and subject to the conditions listed the section above entitled "Special Conditions / Comments".

During the course of the study the protocol may not be modified without prior written approval from the REB except when necessary to remove subjects from immediate endangerment or when the modification(s) pertain to only administrative or logistical components of the study (e.g. change of telephone number). Investigators must also promptly alert the REB of any changes which increase the risk to participant(s), any changes which considerably affect the conduct of the project, all unanticipated and harmful events that oceur, and new information that may negatively affect the conduct of the project and safety of the participant(s). Modifications to the project, information/consent documentation, and/or recruitment documentation, should be submitted to this office for approval using the "Modification to research project" form available at: http://www.rges.uottawa.ca/ethics/application_dwn.asp

Please submit an annual status report to the Protocol Officer 4 weeks before the above-referenced expiry date to either close the file or request a renewal of ethics approval. This document can be found at: http://www.rges.uottawa.ca/ethics/application_dwn.asp

Signature:

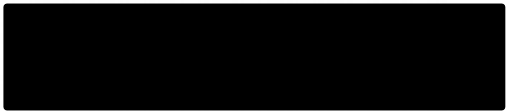

Riana Marcotte

Protocol Officer for Ethics in Research

For Daniel Lagarec, Chair of the Sciences and Health Sciences REB 


\subsection{Study 2 (Data publically available, therefore no approval required)}

From: Ethics [mailto:ethics@uottawa.ca]

Sent: September 27, 2011 3:16 PM

To: Saunders, Travis

Subject: RE: Secondary data sets

Hello Mr. Saunders,

Thank you for your email.

You will in fact need to submit for Secondary use of data for the studies from Quebec and from CHEO. Because the data from United States is publicly available, it will not be necessary to get a certificate for that data.

Secondary use of data refers to the use in research of information originally collected for a purpose other than the current research purpose (p. 62 of the Tri-Council Policy Statement on Ethics in Research).

Along with the submission form, you will need to submit a 5-page research proposal, as well as a permission letter from the original owner of the data indicating that they grant you permission to use the data for your research purposes.

If you have any questions or comments, please do not hesitate to contact me via email or at extension 5387,

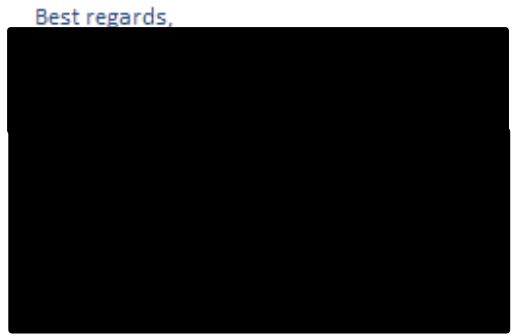




\subsection{Studies $3 \& 4$}

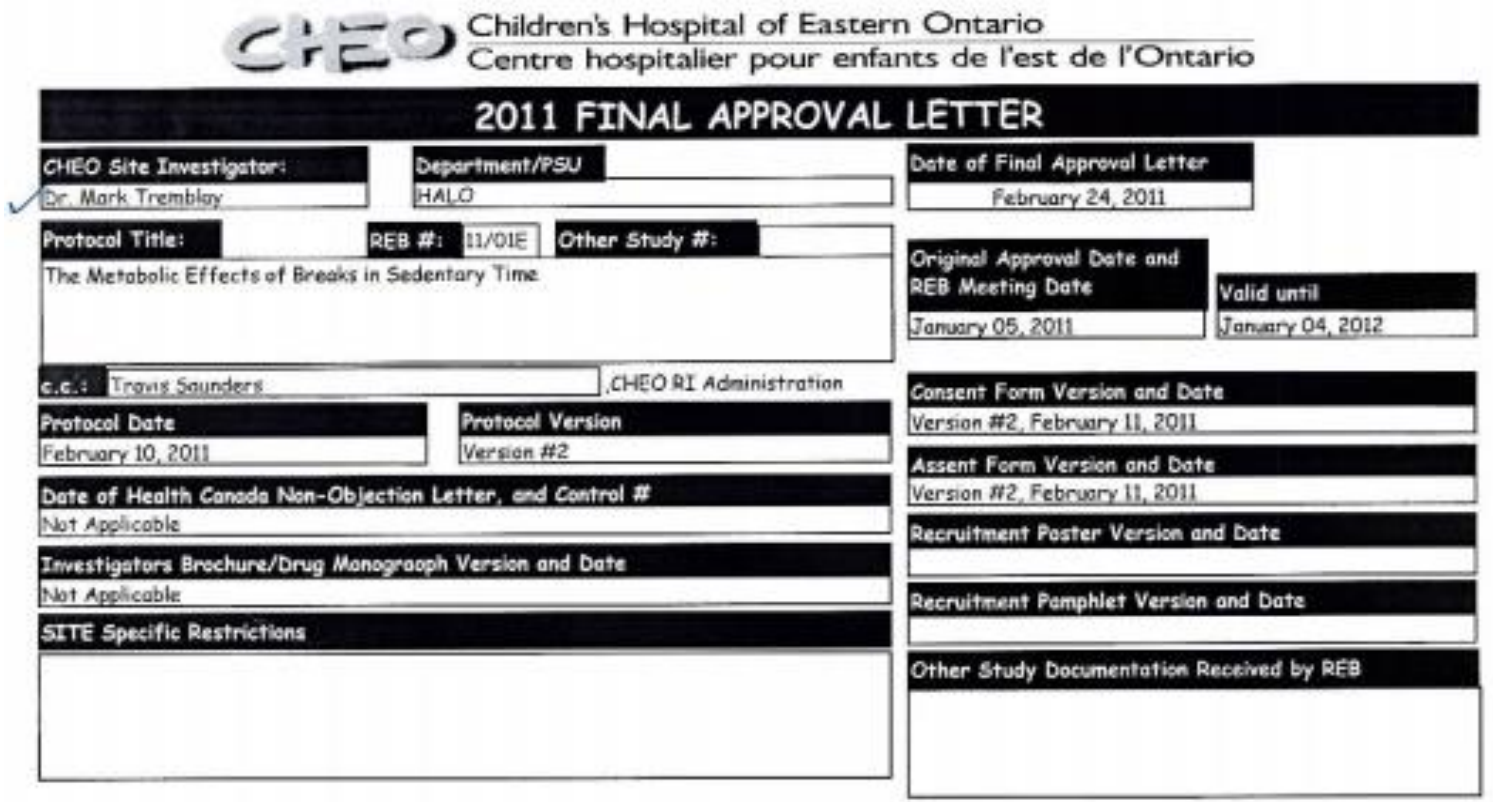

This protocol was approved at a meeting of the CHEO Research Ethics Boond in which the quorum rules were met and only those REB members who were independent of the investigator(s) conducting the study voted on the finol decision.

In fulfilling its mondate, the CHEO REB is guided by: Tri-Council Policy Statement, ICH Good Clinical Practice Proctices: Consolideted Guideline: Applicable laws and regulations of Ontario end Canoda (e.g. Health Canoda Division 5 of the Food and Drug Regulations \& the Food and Drugs Act - Medical Devices Regulations)

Final approval is granted with the understanding that the investigator ogrees to comply with the following requirements - The imestigator mast conduct the study in complance with the protocol and any additional conditions set eut by the Board.

- The imestigator must not implement any deviation from, or changes to, the protocol without the approval of the REB except where necessary to eliminate an inmediate hazard to the research subject, or when the change imvolves only lagistical or odministretive aspects of the study (e. 9. change of telephone number or research staff). As soon as possible, however, the protocol deviation form and, if apprope iete, the proposed protocol amendment(s) should be submitted to the Boord for review.

- The imestigator mast, prier to use, submit to the Board changes to the study documentetian, eg. ehanges to the informed carcent latters recruitaent materials. Should majar revisons to the consent form be made, the investigater ogrees to re-cemerent those subjects who hare originally cansented to the study and who wish to continue on the studty.

- For clinical drug or device trials, imestigators must promptly report to the REB all adverse events that are both serious and unexpected (SAES). For SAE reports on ONEO patients, the inwestigatar must also comply with the hospital-wide Policy regarding. Procedures For Considering Nedical Errur In The Oifferential Diagrosis of Severe Adverse Events (SAE) Associated with the Drugs Administered in a Clinical

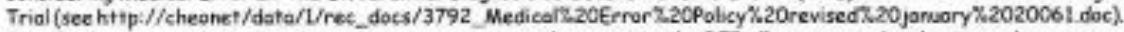

For all other research studies, investigators muat promptly report to the REB all unexpected and untoward occurrences (including the less ar theft of study data and other such privacy breaches)

- Investigators must prometly report to the REB any new information regarding the safety of reseorch subjests (e.g., shanges to the product menogroph or imestigator's brochure fer drug triais). Where available, any reports produced by Data Safety Monitering Boerd should be subaitted to the REB

Imestigators must notify the REB of any study closures (temporary, premoture or permanent), in writing along with an explanatian of the ratiansle for such oction.

- Imestigators must submit an annual renewal report to the AEB 30 dovs priar to the expinstion date stated abave

- Investigators must submit a final report at the conclusion of the study.

- Investigators must provide the Board with French versians of the consent form, unless a waiver has been gronted.

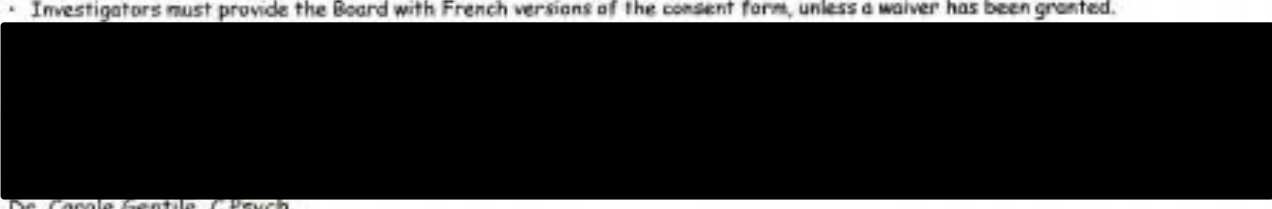

Or. Carole bentile, C.PSych

Chair, Research Ethics Board

CG/smeh 


\section{Université d'Ottawa University of Ottawa Bureau d'éthique et d'integrite de la recherche Office of Research Ethics and lutegnity}

\section{Ethics Approval Notice}

\section{Health Sciences and Science REB}

Principal Investigator / Supervisor / Co-investigator(s) / Student(s)

$\begin{array}{llll}\text { First Name } & \text { L.ast Name } & \text { Affliation } & \text { Role } \\ \text { Mark } & \text { Tremblay } & \text { Health Sciences/Others } & \text { Principal Investigator } \\ \text { Jean-Philippe } & \text { Chaput } & \text { Medicine/Medicine } & \text { Other Collaborator } \\ \text { Rachel } & \text { Colley } & \text { Others / Others } & \text { Other Collaborator } \\ \text { Eric } & \text { Doncet } & \text { Health Sciences / Human Kinetics } & \text { Other Collaborator } \\ \text { Gary } & \text { Goldfield } & \text { Medicine / Medicine } & \text { Other Collaborator } \\ \text { Glen } & \text { Kenny } & \text { Health Sciences / Human Kinetics } & \text { Other Collaborator } \\ \text { Travis } & \text { Saunders } & \text { Health Sciences/Others } & \text { Project Coordinator }\end{array}$

File Number: H 05-11-11

Type of Project: Professor

Title: The Metabolic Impact of Breaks in Sedentary Time

Approval Date (mm/dd/yyyy)

$06 / 13 / 2011$

(Ia: Approval, Ib: Approval for initial stage ouly)

Special Conditions / Comments:

$\mathrm{N} / \mathrm{A}$
Expiry Date (mm/dd/yyyy)

$06 / 12 / 2012$
Approval Type

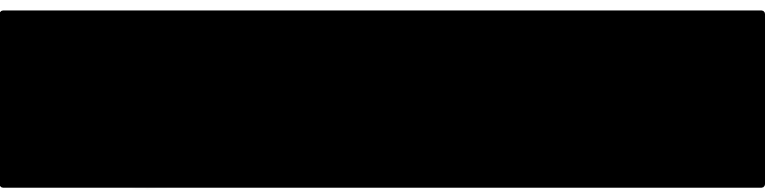




\section{Université d'Ottawa University of Ottawa}

This is to confirm that the University of Ontawa Research Ethics Board identified above, which operates in accordance with the Tri-Council Policy Statement and other applicable laws and regulations in Ontario, has examined and approved the application for ethical approval for the above named research project as of the Ethics Approval Date indicated for the period above and subject to the conditions listed the section above entitled "Special Conditions / Comments".

During the course of the study the protocol may not be modified without prior written approval from the REB except when necessary to remove subjects from immediate endangerment or when the modification(s) pertain to only adninistrative or logistical components of the study (e.g. change of telephone number). Investigators must also promptly alert the REB of any changes which increase the risk to participant(s), any changes which considerably affect the conduct of the project, all unanticipated and harmful events that occur. and new information that may negatively affect the conduct of the project and safety of the participant(s). Modifications to the project. information/consent documentation. and/or recruitment documentation. should be submitted to this office for approval using the "Modification to research project" form available at: http://www.rges.wottawa.ca/ethics/application_dwn.asp

Please submit an anmulal status report to the Protocol Officer 4 weeks before the above-referenced expiry date to either close the file or request a renewal of ethics approval. This document can be found at: http://www.rges uottawa.ca/ethics/application_dwn.asp
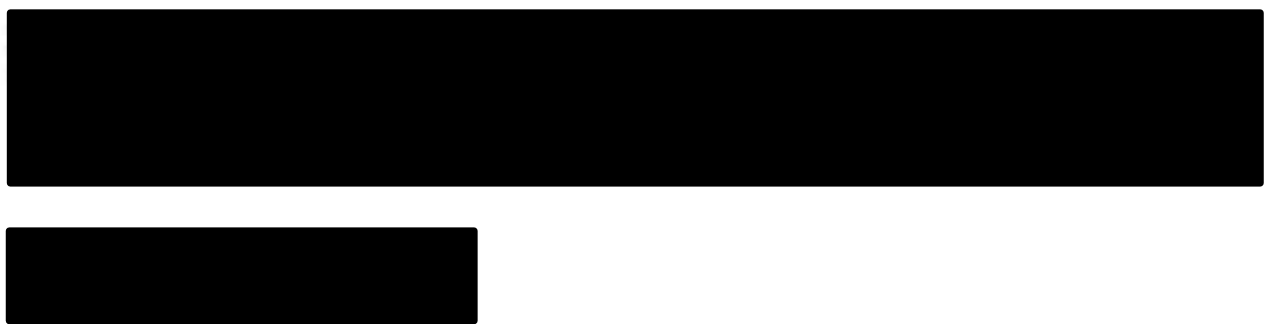

Germain Zongo

Protocol Officer for Ethics in Research

For Dr. Daniel Lagarec, Chair of the Health Sciences and Sciences REB 


\section{Appendix B: Permission for republication}

Chapters 2 and 5 have been accepted for publication in Elsevier journals, which do not require permission in order to include a manuscript in a thesis document (see below). Chapters 3 and 6 are published in journals which allow the author to maintain copyright, therefore permission is not required for these studies either.

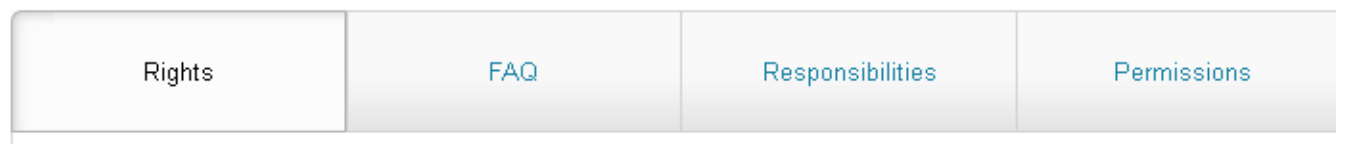

As a journal author, you have rights for a large range of uses of your article, including use by your employing institute or company. These rights can be exercised without the need to obtain specific permission.

How authors can use their own journal articles

Authors publishing in Elsevier journals have wide rights to use their works for teaching and scholarly purposes without needing to seek permission.

Table of Authors' Rights

\begin{tabular}{|c|c|c|c|}
\hline & $\begin{array}{l}\text { Preprint version (with a } \\
\text { few exceptions- see } \\
\text { below *) }\end{array}$ & $\begin{array}{l}\text { Accepted Author } \\
\text { Manuscript }\end{array}$ & $\begin{array}{l}\text { Published Journal } \\
\text { Articles }\end{array}$ \\
\hline $\begin{array}{l}\text { Use for classroom teaching by } \\
\text { author or author's institution and } \\
\text { presentation at a meeting or } \\
\text { conference and distributing } \\
\text { copies to attendees }\end{array}$ & Yes & $\begin{array}{l}\text { Yes with full } \\
\text { acknowledgement of final } \\
\text { article }\end{array}$ & $\begin{array}{l}\text { Yes with full } \\
\text { acknowledgement of final } \\
\text { article }\end{array}$ \\
\hline $\begin{array}{l}\text { Use for internal training by } \\
\text { author's company }\end{array}$ & Yes & $\begin{array}{l}\text { Yes with full } \\
\text { acknowledgement of final } \\
\text { article }\end{array}$ & $\begin{array}{l}\text { Yes with full } \\
\text { acknowledgement of final } \\
\text { article }\end{array}$ \\
\hline $\begin{array}{l}\text { Distribution to colleagues for } \\
\text { their research use }\end{array}$ & Yes & Yes & Yes \\
\hline $\begin{array}{l}\text { Use in a subsequent compilation } \\
\text { of the author's works }\end{array}$ & Yes & $\begin{array}{l}\text { Yes with full } \\
\text { acknowledgement of final } \\
\text { alticte }\end{array}$ & $\begin{array}{l}\text { Yes with full } \\
\text { acknowledgement of final } \\
\text { article }\end{array}$ \\
\hline $\begin{array}{l}\text { Inclusion in a thesis or } \\
\text { dissertation }\end{array}$ & Yes & $\begin{array}{l}\text { Yes with full } \\
\text { acknowledgement of final } \\
\text { article }\end{array}$ & $\begin{array}{l}\text { Yes with full } \\
\text { acknowledgement of final } \\
\text { article }\end{array}$ \\
\hline $\begin{array}{l}\text { Reuse of portions or extracts } \\
\text { from the article in other works }\end{array}$ & Yes & $\begin{array}{l}\text { Yes with full } \\
\text { acknowledgement of final } \\
\text { article }\end{array}$ & $\begin{array}{l}\text { Yes with full } \\
\text { acknowledgement of final } \\
\text { article }\end{array}$ \\
\hline $\begin{array}{l}\text { Preparation of derivative works } \\
\text { (other than for commercial } \\
\text { purposes) }\end{array}$ & Yes & $\begin{array}{l}\text { Yes with full } \\
\text { acknowledgement of final } \\
\text { article }\end{array}$ & $\begin{array}{l}\text { Yes with full } \\
\text { acknowledgement of final } \\
\text { article }\end{array}$ \\
\hline Preprint servers & Yes & $\begin{array}{l}\text { Yes with the specific } \\
\text { written permission of }\end{array}$ & No \\
\hline
\end{tabular}

From: http://www.elsevier.com/journal-authors/author-rights-and-responsibilities 


\title{
Appendix C: Related publications
}

\begin{abstract}
All of the publications in this appendix have been authored or co-authored by Travis Saunders during his PhD studies. Although they are not a part of the thesis itself, they are related to the health impact of sedentary behaviour, and are therefore included to provide additional context to the thesis. All of these papers have been published in journals with Creative Commons Attribution licenses, and therefore do not require permission for republication.
\end{abstract}




\title{
Review Article
}

\section{Acute Sedentary Behaviour and Markers of Cardiometabolic Risk: A Systematic Review of Intervention Studies}

\author{
Travis J. Saunders, ${ }^{1,2}$ Richard Larouche, ${ }^{1,2}$ Rachel C. Colley, ${ }^{1}$ and Mark S. Tremblay ${ }^{1,2,3}$ \\ 'Healthy Active Living and Obesity Reseurch Group, Children's Hospital of Eastern Ontario Research Institute, \\ Ortami, ON, Canada KlH 8LI \\ School of Human Kinetics, University of Ottami, Ottiwi, ON, Canada K1N 6N5 \\ 'Department of Pediatrios, University of Ottama, Ottaun, ON, Canada KIN 6N5 \\ Correspondence should be addressed to Travis J. Saunder \\ Received 24 January 2012; Accepted 28 March 2012 \\ Academic Editor: Maria Luz Fernandez \\ Copyright 2012 Travis J. Saunders et al. This is an open access article distributed under the Creative Commons Attribution \\ License, which permits unrestricted use, distribution, and reproduction in any medium, provided the original work is properly \\ cited. \\ North Americans spend half their waking hours engaging in sedentary behaviour. Although several recent internentions suggest \\ that short bouts of uninterrupted sedentary behaviour may result in acute increases in cardiometabolic risk, this literature has not \\ been reviewed systematically. This study performed a systematic review of the impact of uninterrupted sedentary behaviour lasting \\ 57 days on markers of cardiometabolic risk (insulin sensitivity, glucose tolerance, and fasting insulin, glucose, and lipid levels) \\ in humans. Interventions were identified through systematic searches of Medline and Embuse and screened by 2 independent \\ reviewers. $A$ total of 25 interventions were identified that examined the impact of imposed sedentary behaviour on biomarkers of \\ interest. The majority of these studies focused on bealthy young men, with very little identified research on females or other age \\ groups. We found consistent, moderate quality evidence that uninterrupted sedentary behaviour $\leq 7$ days results in moderate and \\ deleterious changes in insulin sensitivity, glucose tolerance, and plasma triglyceride levels. In contrast, there is inconsistent, very \\ low-quality evidence linking uninterrupted sedentary behaviour with changes in insulin, glucose, and HDL-and LDL-cholesterol \\ levels. These findings saggest that uninterrupted bouts of sedentary behaviour should be avoided in order to prevent or attenuate \\ transient increases in metabolic risk.
}

\section{Introduction}

High levels of chronic sedentary behaviour are associated with increased risk of obesity $[1,2]$, diabetes $[1,3]$, cardiovascular disease $[4-6]$, some cancers $[7]$, and even mortality [3-5]. For example, it has been reported that sedentary behaviour is prospectively associated with increased risk of all-cause and cardiovascular disease mortality, and that these associations remain significant after control for physical activity, diet, and smoking [4]. These findings suggest that sedentary behaviour should not be viewed as simply the lack of physical activity but may instead represent an independent and distinct risk factor for chronic disease.

In addition to the health impact of chronic sedentary behaviour, recent evidence suggests that increases in metabolic risk may be apparent following bouts of uninterrupted sedentary behaviour lasting just a few days in length [8-11]. Stephens et al, reported that a single day of uninterrupted sitting resulted in a $39 \%$ reduction in whole boxty insulin action in healthy adults [11]. Similarly, Hamburg et al. observed that 5 days of continuous bed rest produced deleterious changes in cholesterol, triglyceride, glucose, and insulin levels and reduced insulin sensitivity [10]. These findings are supported by work in animal models, which suggest that just 6-24 hours of sedentary behaviour results in significant reductions in lipoprotein lipase activity [12] and insulin sensitivity $[13,14]$ in skeletal muscle.

Although several narrative reviews have discussed the acute changes in metabolic risk following short-term exposure to uninterrupted sedentary behaviour $[8,9,15,16]$, 
the published literature in this area has yet to be examined systematically. Therefore, we conducted a systematic review examining the impact of uninterrupted sedentary behaviour lasting $\leq 7$ days (operationally defined as an "acute" bout) on insulin sensitivity, glucose tolerance, and lipid, glucose, and insulin levels in humans.

\section{Methods}

2.1. Study Criteria. To be included in this paper, a study had to examine at least one of the following risk markers in humans: insulin sensitivity, glucose tolerance, or fasting insulin, glucose, or lipid levels. Uninterrupted sedentary behaviour had to be imposed by the researchers for a period lasting 7 days or less. Studies examining longer (e.g., chronic) bouts of sedentary behaviour were excluded as it was felt that it would be inappropriate to consider the impacts of both acute and chronic sedentary behaviour in a single systematic review, given the large amount of heterogeneity that this would introduce into the methodologies of included studies. Only intervention studies (both randomized and nonrandomized) that imposed on participants a controlled bout of sedentary behaviour were included in this paper.

For the purposes of this paper, sedentary behaviour was defined as a distinct class of waking behaviours characterized by little physical movement and low-energy expenditure ( $\leq 1.5 \mathrm{METs}$ ), as well as a sitting or reclining posture [9]. Eligible forms of sedentary behaviour included sitting, bed rest (head-up, horizontal, and head-down), and casting (e-g, having one or more legs immobilized in a cast). There were no restrictions placed on the age or sex of participants. Only articles published in English or French were included in the present review, and no limits were placed on the date of publication. The review methodology was prospectively registered in PROSPERO (Registration number: CRD42011001431).

2.2. Search Strategy: Literature searches were performed using Ovid Medline and Ovid Embase in March of 2012. The search strategy was created with the help of a research librarian and run by TIS (see Algorithm 1). Potentially relevant articles were also identified by 6 key informants and through the authors' personal reference libraries. Articles were extracted as text files from the Ovid interface and imported into Reference Manager (Thompson Reuters, San Fransisco, CA, USA). Duplicate articles were first removed using the Ovid interface, and any remaining duplicates were removed manually. Once imported into Reference Manager. conference abstracts were also removed from the database.

Titles and abstracts of articles identified through the search were reviewed by two authors (T. I. Saunders and R. Larouche) using Reference Manager. Any articles identified as being potentially relevant by either reviewer were obtained for further screening. The full text of these articles was then reviewed independently by TJS and RL to determine whether the article met the a priori review inclusion criteria. All decisions at this stage were made by consensus and any discrepancies between the two reviewers were resolved through discussion. In this paper consensus was reached for all included articles.

23. Data Extraction and Analysis. Data was extracted by T. J. Saunders and verified by R. Larouche. Information was extracted regarding study design (year, methodology, country, number of participants, duration of sedentary behaviour. age), modality of sedentary behaviour, risk factors examined, and main findings. Reviewers were not blinded to the authors or journals when extracting data. The primary summary measure was the mean difference in each outcome measure (or mean change in nonrandomized interventions) following exposure to acute sedentary behaviour. Where possible, effect sizes were calculated using Cohen's $d$. We defined an effect size of $\leq 0.20$ as small, an effect of $0.21-0.80$ as moderate, and effects $\geq 0.81$ as large. For the purposes of this paper, positive effect sizes represent increased cardiometabolic risk (e.g-, increased fasting triglyceride levels), while negative effect sizes represent reduced risk.

Following data extraction it became clear that the interventions included in the present paper were very heterogeneous in terms of the length of exposure, the type of sedentary behaviour that was examined, and even the measurement of individual risk factors (e.g., insulin sensitivity was assessed using HOMA, QUICKI, whole body insulin action, oral glucose tolerance tests, and hyperinsulinemic clamps). Thus, we believe that meta-analyses or pooling of data across studies would be inappropriate and have therefore performed a qualitative synthesis of the evidence instead.

Forest plots were created using Review Manager 5.1 (The Nordic Cochrane Centre, The Cochrane Collaboration) to display the relationship between sedentary behaviour and each outcome of interest. Studies assessing glucose tolerance and insulin sensitivity employed a wide range of methodologies and units of measurement, and plots for these outcomes are therefore presented as percent mean difference, while all other outcomes are presented as mean difference with $95 \%$ confidence interval. Studies which did not provide raw data were not included in forest plots.

24. Quality of Evidence. The risk of bias and strength of evidence from individual studies was assessed using the Downs and Black Checklist [17], This 27 point checklist assesses the strength of reporting, external validity, internal validity, and power. As some questions are worth more than one point, the maximum score that a study can receive is 32 .

The quality of evidence for each outcome was assessed as high, moderate, low, or very low using the GRADE approach [18]. In this approach, randomized trials begin as highquality evidence and observational studies begin as lowquality evidence. For the purposes of this paper, nonrandomized interventions were considered as observational studies. Following the initial rating based on study design, the quality of evidence was then rated up or down for apparent risk of bias, imprecision, inconsistency, indirectness, of suspicion of publication bias. Risk of bias was assessed using Review Manager Version 5.1 (The Nordic Cochrane Centre, The 


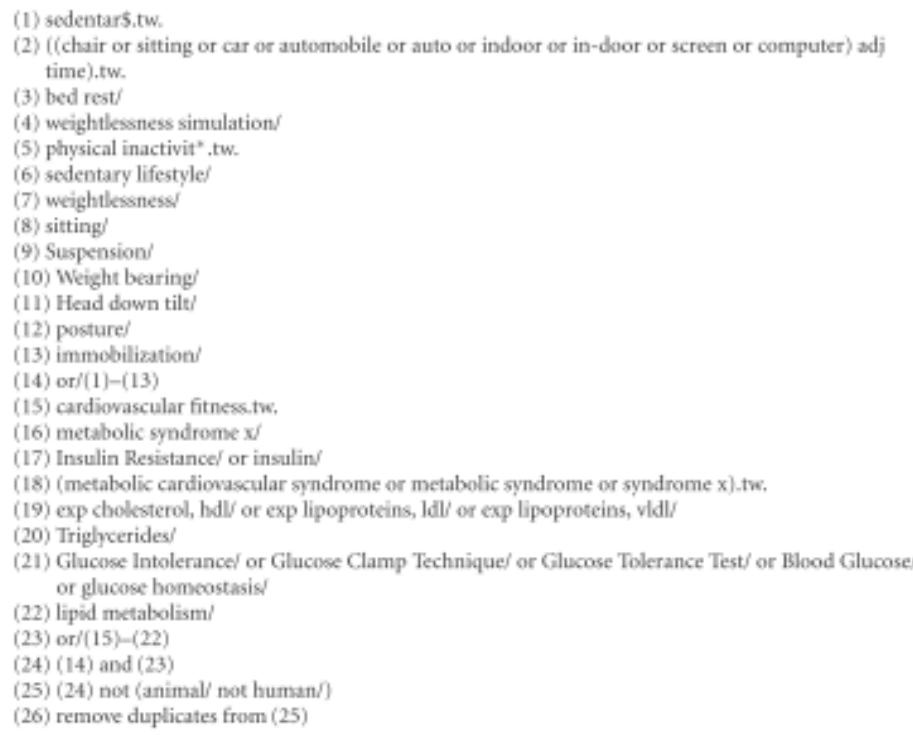

ALCORITHM 1: Medline search strategy.

Cochrane Collaboration), and GRADE was assessed using GRADEpro Version 3.6 (GRADE Working Group).

\section{Results}

3.1. Description of Studies. After deduplication and the removal of conference abstracts the search strategy retrieved 5,670 articles for initial screening (Figure 1). To this, 16 addi. tional articles that were identified through key informants were added, bringing the total number of potential articles to 5,686. Initial screening of titles and abstracts identified 85 articles that received a detailed assessment of the full text article. Reasons for excluding studies included an ineligible exposure ( $\mathrm{e}, \mathrm{g}, \mathrm{s}$ the bout of sedentary behaviour exceeded 7 days, or simply investigated the impact of reducing structured physical activity in active individuals, without actually imposing sedentary behaviour) $(n=26)$, the article being written in a language other than English or French $(n-12)$, ineligible outcome $(n-7)$, the article being a review or commentary $(n=10)$, and "other" $(n=2)$. Some articles were excluded for multiple reasons.

A total of 29 articles reporting data from 25 independent interventions met all inclusion criteria and are presented in the current review. Nineteen of the identified interventions were nonrandomized trials, 4 were randomized crossover studies (e.g-, participants served as their own controls), and 2 were randomized controlled trials. The studies included a total of 368 participants (309 males and 59 females). who were recruited from 12 countries across North America (USA), Europe (Denmark, France, Bulgaria, Russia, Greece, Sweden, Poland, and Slovakia, Norway), Asia (Japan), and Oceania (Australia). Participants ranged from 18 to 72 years of age, although the average age of participants was under 35 years for all but 3 studies, and under 30 for all but 7 studies. Sixteen studies employed head-up or horizontal bed rest, 5 employed head-down bed rest, 4 employed sitting. and one employed casting (one employed both sitting and head-down bed rest). The smallest studies had 5 participant $[19,20]$ and the largest had $38[21]$. The mean number of participants per study was $15,1 \pm 10.1$, and the median was 10 .

Three studies examined the impact of 2,4 , and 5 hours of uninterrupted sedentary behaviour on biomarkers of interest, respectively; all other studies examined the impact of 1 day or more. Six studies examined the impact of 1 day of sedentary behaviour, 4 examined 2 days, 7 examined 3 days, 2 examined 4 days, 6 examined 5 days, 2 examined 6 days, and 8 examined the impact of 7 days of sedentary behaviour ( 6 studies collected data at multiple time-points). Characteristics of individual studies are presented in Table 1 .

3.2. Fasting lnsulin. Two randomized crossover studies ( $n-$ 22) $[11,22]$ and 12 nonrandomized intervention studies ( $n=185\rangle[10,21,23-32]$ examined the impact of sedentary behaviour on fasting insulin levels (Figure 2). Neither 


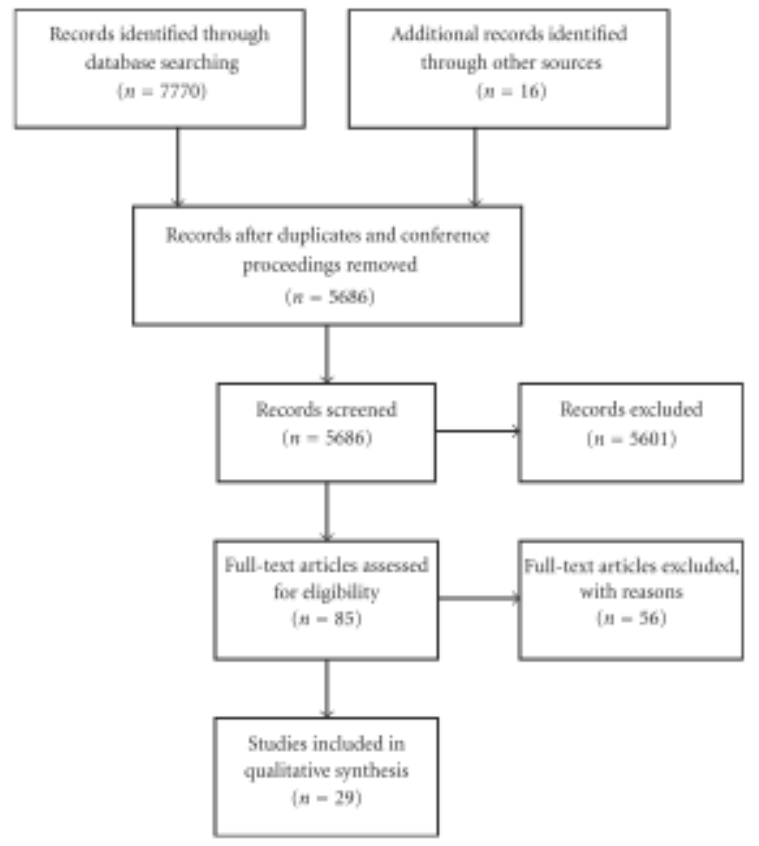

Figure 1: Flow of articles through the search process.

randomized crossover study obsenved significant changes in insulin levels as a result of uninterrupted sedentary behaviour, despite having effect sizes in the moderate range. Stephens et al. [11] reported that fasting insulin levels were $47.6 \pm 11.6 \mathrm{pmol} / \mathrm{L}$ following one full day (17 hours) of uninterrupted sitting, compared to $39.3 \pm 16.3 \mathrm{pmol} / \mathrm{L}$ following a day that included as little sitting as possible ( 6 hours of sitting spread throughout the day) in a group of 12 healthy young adults. Duran-Valdez et al. [22] also observed nonsignificant increases following 2 days of strict bed rest in both healthy participants $(71.4 \pm 42.6$ versus $84.1 \pm$ $36.4 \mathrm{pmol} / \mathrm{L})$ and those with type 2 diabetes $(79.2 \pm 50.3$ versus $106.3 \pm 46.6 \mathrm{pmol} / \mathrm{L}$ ).

Three nonrandomized interventions reported significant increases in insulin levels ranging from 26 to $47 \%$ following uninterrupted sedentary behaviour $[10,23,28]$ while 8 reported no change $[21,24-27,29,30]$. The interventions that observed a change in insulin levels tended to impose uninterrupted sedentary behaviour for a longer period of time than those that found no change $(6.0 \pm 1.0$ days versus $3.9 \pm 2.6$ days $)$, although the mean number of participants $(14.0 \pm 7.0$ versus $16,4 \pm 13.3)$ and the quality of the studies did not appear to differ across the interventions. Effect sizes for these nonrandomized interventions ranged from -0.16 to 0.95 , with all but one study reporting effect sizes in the small and moderate ranges,
Given the aforementioned evidence from both randomized and nonrandomized interventions, we conclude that an acute bout of uninterrupted sedentary behaviour may result in a small-to-moderate increase in fasting insulin levels. However, the inconsistency of this effect and the lack of a statistical significance in randomized interventions leads us to conclude that the quality of this evidence is very low (Table 2),

3.3. Fasting Glucase. Two randomized crossower studies ( $n=$ 22) $[11,22]$, one randomized controlled trial $(n=20)$ [33], and 14 nonrandomized intervention studies $(n-149)$ $(10,20,23-30,34-36)$ examined the impact of uninterrupted sedentary behaviour on fasting glucose levels. The one randomized controlled trial reported that, in comparison to ambulatory controls, plasma glucose levels were elevated by $34 \%$ following 7 days of uninterrupted bed rest [33]. The effect size in this randomized controlled trial was greater than 1 , signifying a large effect. In contrast, neither randomized crossover study reported any change in fasting glucose levels following uninterrupted sedentary behaviour [11,22], One of these randomized crossover studies reported a moderate effect size of $0.43,[11]$, while the other reported small effect sizes of 0.07 and 0.14 in healthy participants and those with type 2 diabetes, respectively [22]. 


\begin{tabular}{|c|c|c|c|c|c|c|c|c|c|c|c|}
\hline Reference & Design & First author & Year & Country & $n(\mathrm{M} / \mathrm{F})$ & Age range & Mean age & Modality & Duration & Outcomes & $\begin{array}{c}\text { Downs and } \\
\text { Black }\end{array}$ \\
\hline [37] & RCT & Yaroshenko & 1998 & Greece & $30(30 / 0)$ & $22-26$ & 24.8 & BR & 7 days & TG & 24 \\
\hline [33] & RCT & Zorbas & 1999 & Bulgaria & $30(30 / 0)$ & $22-26$ & 24.3 & $\mathrm{BR}$ & 7 days & FG & 24 \\
\hline$[22]$ & $\mathrm{RCO}$ & Duran-Valdez & 2008 & USA & $10(2 / 8)$ & $24-72$ & 46.2 & $\mathrm{BR}$ & 2 days & F, FG, IS & 21 \\
\hline [11] & $\mathrm{RCO}$ & Stephens & 2010 & USA & $12(6 / 6)$ & $19-32$ & 26.1 & SIT & $1 \mathrm{day}$ & F, FG, is & 24 \\
\hline [39] & $\mathrm{RCO}$ & Dunstan & 2012 & Australia & $19(11 / 8)$ & $45-65$ & 53.8 & SrT & 5 hours & IS, GT & 27 \\
\hline [42] & $\mathrm{RCO}$ & Nygaard & 2009 & Norway & $13(0 / 13)$ & $>50$ & & SIT & 2 bours & GT & 23 \\
\hline [21] & NT & Alibegovic & 2010 & Denmark & $38(38 / 0)$ & & 25.0 & BR & 7 days & FI & 23 \\
\hline [31] & NT & Alibegovic & 2009 & Denmark & $33(33 / 0)$ & & 25,6 & BR & 7 days & FI & 23 \\
\hline$[23,47]$ & NT & Blanc & 2000 & France & $16(8 / 8)$ & & 30.2 & HDBR & 6 days & $\underset{\text { GT }}{\mathrm{FI}, \mathrm{FG}, \mathrm{IS},}$ & 23 \\
\hline [35] & NT & Dolkas & 1977 & USA & $7(7 / 0)$ & $19-22$ & 20.0 & $\mathrm{BR}$ & 4 days & FG & 21 \\
\hline [25] & NT & Barbe & 1999 & France & $8(8 / 0)$ & $23-31$ & 27.1 & HDBR & 5 days & $\mathrm{FI}, \mathrm{FG}$ & 21 \\
\hline [10] & NT & Hamburg & 2007 & USA & $20(14 / 6)$ & & 30.7 & $\mathrm{BR}$ & 5 days & $\begin{array}{c}\mathrm{F}, \mathrm{FG}_{\mathrm{TG}} \text {, }\end{array}$ & 23 \\
\hline [20] & NT & Katkow & 1979 & Russia & $5(5,0)$ & & 34.0 & BR & 5 days & FG & 17 \\
\hline [41] & $\mathrm{NT}$ & Kïilerich & 2011 & Denmark & $6(6 / 0)$ & $22-36$ & 28.7 & BR & 7 days & IS, GI & \\
\hline [36] & NT & Ksinantova & 2002 & Slovakis & $15(15 / 0)$ & & 34.0 & HDBR & 4 days & FG & 20 \\
\hline [29] & $\mathrm{NT}$ & Lipman & 1972 & USA & $7(7 / 0)$ & $18-20$ & & BR & 3 days & Fl, IS, GT & 18 \\
\hline [32] & NT & Kanikowska & 2010 & Japan & $8(8 / 0)$ & & 27.0 & HDBR & 5 days & $\mathrm{Fl}, \mathrm{FG}, \mathrm{IS}$ & \\
\hline$[27,40]$ & NT & Mikines & 1989 & Denmark & $6(6 / 0)$ & & 25.0 & $\mathrm{BR}$ & 7 days & $\underset{\text { GT }}{\mathrm{Fl}, \mathrm{FG}, \mathrm{IS},}$ & 20 \\
\hline [30] & NT & Moro & 2007 & France & $8(8 / 0)$ & $22-27$ & 23,0 & $\begin{array}{c}\text { HDBR, } \\
\text { SIT }\end{array}$ & 4 hours & $\mathrm{FI}, \mathrm{FG}$ & 20 \\
\hline [34] & NT & Navasiolava & 2010 & Russia & $8(8 / 0)$ & & 23,0 & $\mathrm{BR}$ & 7 days & $\begin{array}{c}\text { FG, TG, } \\
\text { HDL., } \\
\text { LDL }\end{array}$ & 22 \\
\hline [26] & NT & Nygren & 1997 & Sweden & $6(6 / 0)$ & & 24.1 & $\mathrm{BR}$ & $1 \mathrm{day}$ & $\mathrm{Fl}, \mathrm{FG}, \mathrm{IS}$ & 22 \\
\hline [19] & NT & Richter & 1989 & Denmark & $5(5,0)$ & $22-24$ & & CAST & 7 days & IS & 20 \\
\hline $\begin{array}{l}{[24,48,} \\
49]\end{array}$ & $\mathrm{NT}$ & Smorawinski & 1996 & Poland & $29(29 / 0)$ & & 20.10 & BR & 3 days & IS, GT & 19 \\
\hline [28] & NT & Stuart & 1988 & USA & $6(6 / 0)$ & $21-28$ & 23.0 & $\mathrm{BR}$ & 7 days & $\underset{\text { GT }}{\mathrm{F}_{3}, \mathrm{FG}, \mathrm{IS},}$ & 22 \\
\hline$[38]$ & NT & Yanagibori & 1997 & Japan & $23(13 / 10)$ & $19-25$ & & BR & 3 days & $\begin{array}{c}\text { IS, GT, } \\
\text { TG, HDL, } \\
\text { LDL. }\end{array}$ & 22 \\
\hline
\end{tabular}

RCT: randomized controlled trial; ROO: rasdomized crossower, NT: nonrandomized trial; M: male; F: female; HDER: heud-down bed rest; 22BR: borizontal or head-up bed rest; STT: sitting; CAST; casting; FG: fasting glusose; FE: fasting insulins: TG: trighroerides; HDL; HDL-cholesterol; LDL; LDL-cholesterol; IS; insulin sensitivity; GT: glucose tolerance.

Whes an intervention was described in more than one paper, the wathor name and year are taken from the earliest publication.

One nonrandomized intervention observed a significant increase in glucose levels of moderate size [10], one reported a significant reduction of moderate size [36], and one intervention observed moderate and large reductions in males and females, respectively, although this change was only significant in females [23]. The 11 other intervention studies did not observe any significant change in fasting glucose levels following uninterrupted sedentary behaviour [20,24$30,32,34,35]$. The effect sizes among these 11 studies ranged from -1.21 to 0.47 .

Given the evidence provided by 17 separate intervention studies, we conclude that an acute bout of uninterrupted sedentary behaviour may result in a small-to-moderate increase in fasting glucose levels. However, the high level of inconsistency from both randomized and nonrandomized interventions leads us to conclude that this evidence is of very low quality.

3.4. Fasting Triglycerides, One randomized controlled trial $(n-30)[37]$ and 3 nonrandomized interventions $(n-51)$ $[10,34,38]$ assessed the impact of uninterrupted sedentary behaviour on fasting triglyceride levels (Figure 3). The randomized controlled trial [37] exposed 20 men to one week 


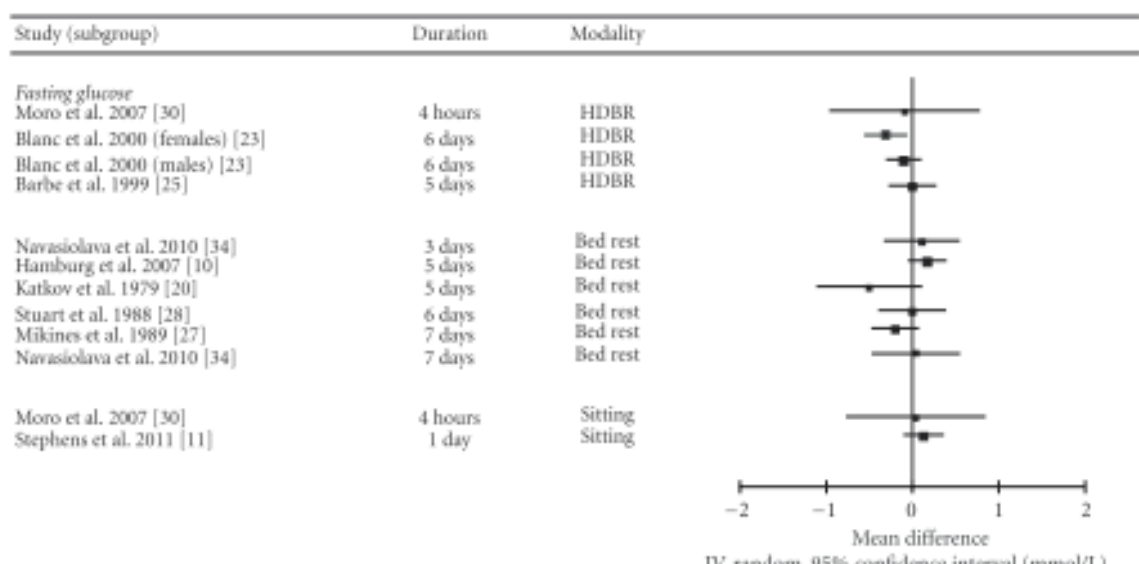

Fasting inewlis

Moro ef al. 2007 [30]

Barbe et al. 1999 [25]

Blanc et al. 2000 (females) (23)

Blanc ef al. 2000 (maks) |23)

4 hours

5 days

6 dap

6 dayk

Duran-Valdez et al. 200 (T2D) [22]

Duran-Valdez et al. 2008 (healthy) [22]

2 doys

2 dovs

tipman es al. 1972 [29]

Hamburg et al. 2003 [10]

3 days

5 days

Stuart et al. 1988 [28]

4 hours

Moro et al. 2007 [50]

Stephens et al. 2011 [11]

1 day
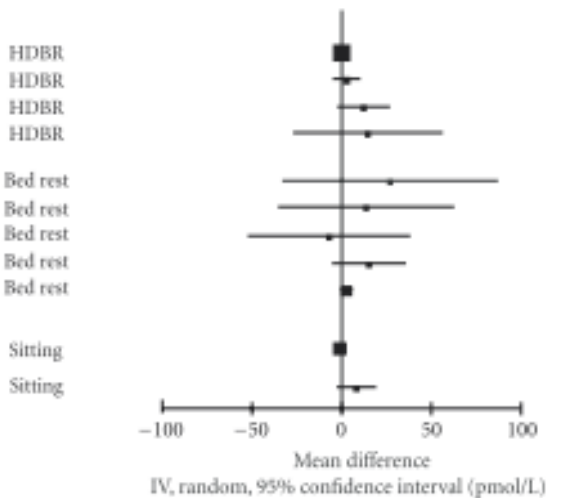

Figure 2: Forest plot of mean differences of fasting glucose and insulin values between sedentary behaviour and control conditions (sedentary behaviour-control).

of bed rest and assessed triglyceride levels on days 1,3 , and 7. The 20 men in the experimental group were further split into two groups of 10-those who knew when their bed rest would begin (acute bed rest), and those who were not told when it would begin (rigorous bed rest). In comparison to the control group, triglyceride levels were significantly elevated by $30.2 \%$ in the acute group after one day, although no change was observed in the rigorous group. Following 3 days of bed rest, triglyceride levels were elevated by $15.2 \%$ and $23.6 \%$ in the acute and rigorous bed rest groups, respectively. At the completion of 1 week of bed rest, triglyceride levels remained elevated by $36.8 \%$ and $31.9 \%$ in the acute and rigorous bed rest groups in comparison to the control group. The effect size for sedentary behaviour in this intervention was above 1 for both intervention groups on days 1,3 , and 7 , indicating a large effect.
The three nonrandomized interventions also found that acute sedentary behaviour resulted in significant increases in triglyceride levels [10, 34, 38]. Hamburg et al. [10] reported that triglyceride levels were elevated by $34.8 \%$ following 5 days of bed rest in 20 healthy men and women. Navasiolava et al. [34] observed that although no change in triglyceride levels was observed following 3 days of acute sedentary behaviour in a group of 8 male participants, triglyceride levels were $58.9 \%$ higher than baseline on day 7 . Finally, Yanagibori et al. [38] found that triglyceride levels were elevated by $38.1 \%$ following 3 days of bed rest in men, but not women. With the exception of male participants in one study [38], the effect sizes reported in these nonrandomized interventions were all moderate to large.

Given the large and relatively consistent changes in triglyceride levels reported by both a randomized controlled 
TA=LE 2: Summary of key evidence.

\begin{tabular}{lcccc}
\hline Risk factor & Number of studies & Number of participants (M/F) & Size of effect & Quality of evidence \\
\hline Insulin sensitivity & 11 & $161(118 / 43)$ & Moderate-to-Large & Moderate quality \\
Triglycerides & 4 & $81(65 / 16)$ & Moderate-to-Large & Moderate quality \\
Glucose tolerance & 6 & $119(83 / 36)$ & Moderate-to-Large & Moderate quality \\
HDL-cholesterol & 3 & $51(35 / 16)$ & Moderate & Very low quality \\
Fasting insulin & 14 & $207(187 / 20)$ & Small-to-Moderate & Very low quality \\
Fasting glucose & 17 & $191(163 / 28)$ & Small-to-Moderate & Very low quality \\
LDL-cholesterol & 2 & $28(22 / 6)$ & Moderate & Very low quality \\
\hline
\end{tabular}

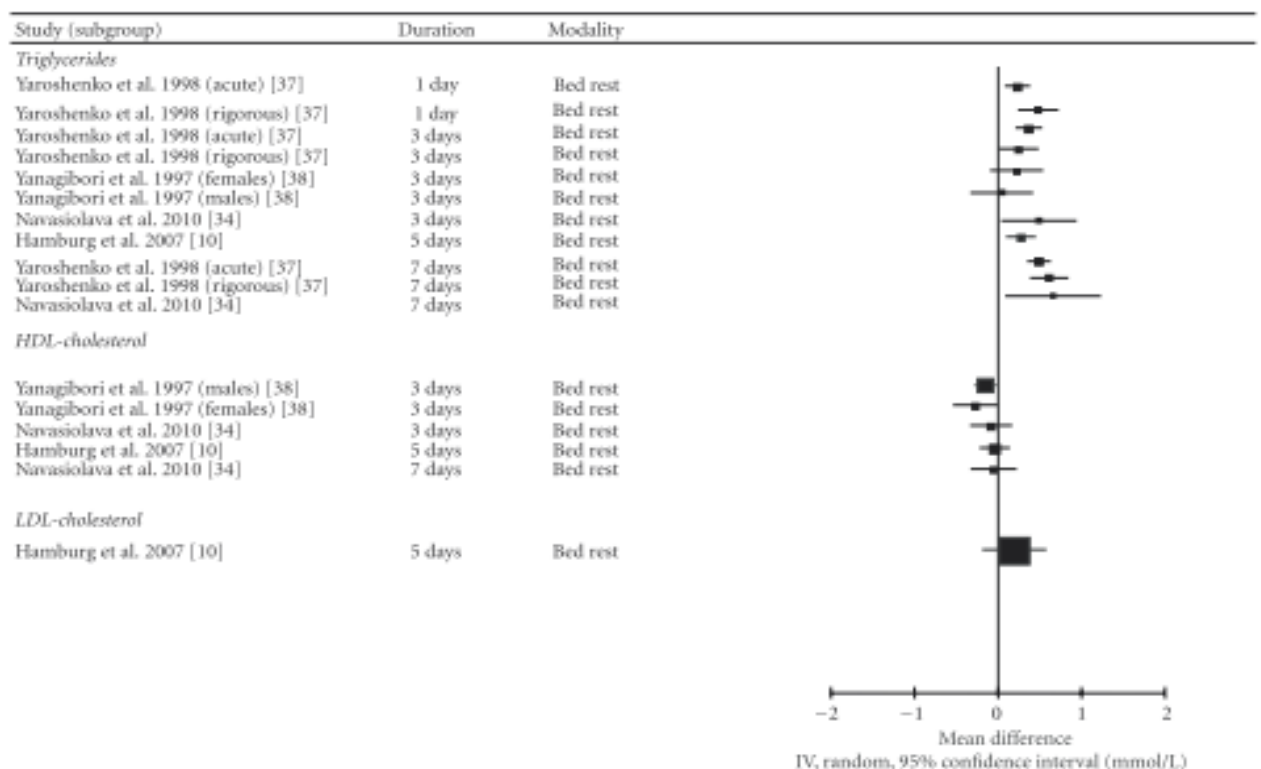

Figure 3: Forest plot of mean differences of fasting lipid levels between sedentary behaviour and control conditions (sedentary behaviourcontrol).

trial and nonrandomized interventions, we conclude that acute bouts of uninterrupted sedentary behaviour result in a moderate-to-large increase in circulating triglyceride levels and that the available evidence is of moderate quality.

3.5. Fasting HDL-Cholesterol. Three nonrandomized interventions $(n=51)[10,34,38]$ reported on the effect of uninterrupted sedentary behaviour ranging from 3 to 7 days on HDL-cholesterol levels. Two interventions reported nonsignificant reductions in HDL-cholesterol levels following sedentary behaviour $[10,34]$ while one study $[38]$ reported significant reductions of $11.5 \%$ and $19.3 \%$ in men and women, respectively, following 3 days of bed rest. The effect sizes in these studies ranged from 0.09 to 0.84 , suggesting that acute bouts of uninterrupted sedentary behaviour may result in moderate reductions in HDL-cholesterol levels. However, given the inconsistency of these findings and the lack of data from randomized interventions, we conclude that the available evidence is of very low quality.

3.6. L.DL-Cholesterol. Two nonrandomized interventions ( $n$ - 28) $[10,34]$ examined the relationship between uninterrupted sedentary behaviour and changes in LDL-cholesterol levels following $3[34], 5[10]$, and 7 [34] days of sedentary behaviour. Although the studies reported moderatesized increases in LDL-cholesterol levels at all time points, 


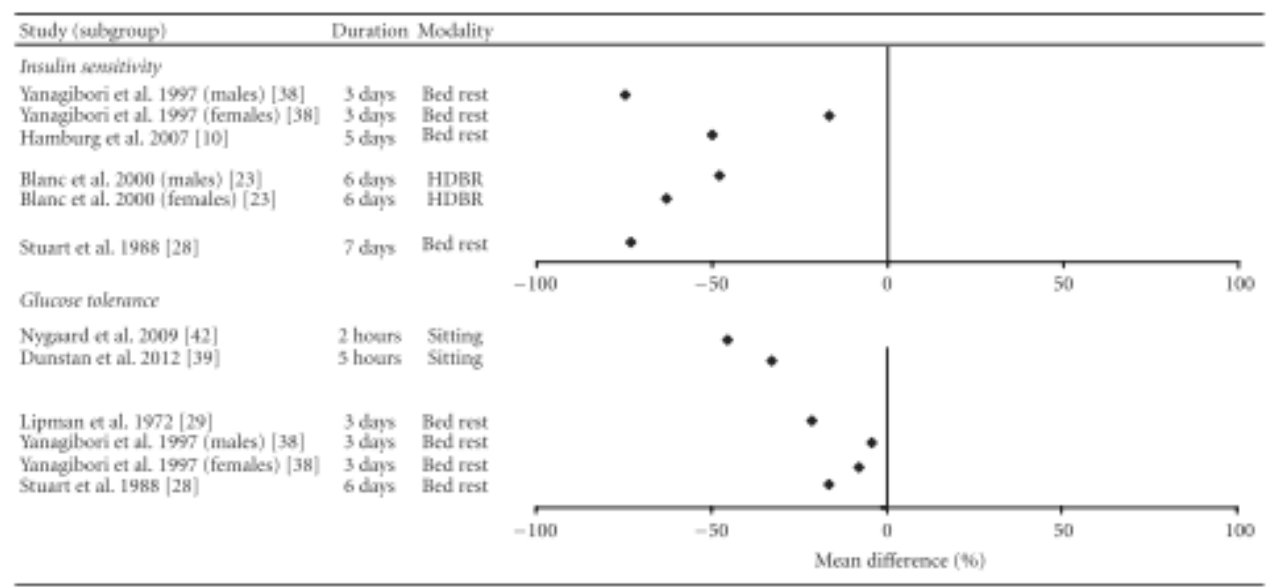

Fugura. 4: Forest plot of percent mean differences of insulin sensitivity and glucose tolerance between sedentary behaviour and control conditions (sedentary behaviour-control).

none of these increases were statistically significant. Thus, while the available evidence suggests that an acute bout of uninterrupted sedentary behaviour may result in a moderate increase in LDL-cholesterol levels, the quality of this evidence is very low.

3.7. Insulin Sensitivity. Three randomized crossover studies $(n=41)[11,22,39]$ and 10 nonrandomized interventions $(n=120)[10,19,23,24,28,29,32,38,40,41]$ examined the relationship between acute bouts of uninterrupted sedentary behaviour and measures of insulin sensitivity in healthy adults (Figure 4). The measures employed included HOMA $[10,32,41]$, QUICKI [22], insulin-stimulated glucose uptake [11], insulin sensitivity index [10], insulin area underthe-curve (AUC) during oral glucose tolerance tests or standardized meals $[23,24,28,38,39,41]$, and hyperinsulinemic euglycemic clamps $[19,28,40]$. The crossover studies measured insulin sensitivity during 5 hours of sedentary behaviour [39], as well as before and after $1[11,22]$ and 2 [22] days of sedentary behaviour. The nonrandomized interventions assessed insulin sensitivity before and after 3 $[24,29,38], 5[10], 6[23]$, and $7[19,28,40,41]$ days of sedentary behaviour.

Two of the three randomized crossover studies [11, 39] reported that uninterrupted sedentary behaviour had a deleterious effect on insulin sensitivity. Stephens et al. reported that insulin-stimulated glucose uptake was $39 \%$ lower following a day of acute sitting in a group of 12 healthy adults, in comparison to a day that minimized sitting [11]. Similarly, Dunstan et al. reported that the insulin AUC following a standardized meal was increased by $30 \%$ following 5 hours of uninterrupted sitting in a group of 19 overweight adults, in comparison to 5 hours of sitting which was broken up with periodic light-intensity walk breaks
[39]. The third crossover study [22] reported that 1 day of sedentary behaviour resulted in a nonsignificant reduction in QUICKI scores in 5 healthy adults and a nonsignificant increase in 5 adults with type 2 diabetes. $A$ significant reduction in insulin sensitivity was observed following two days of bed rest in participants with type 2 diabetes, but not in healthy adults. The significant reduction in participants with type 2 diabetes following 2 days of bed rest was of moderate size, while the nonsignificant reduction in healthy participants was small.

Eight of the 10 nonrandomized trials reported significant reductions in insulin sensitivity ranging from $12.5 \%$ to $100 \%$ following uninterrupted sedentary behaviour. For example. Hamburg et al. [10] reported that HOMA insulin sensitivity was reduced by $50 \%$ following 5 days of bed rest in 20 healthy adults, while the insulin sensitivity index was reduced by $12.5 \%$ in the same group of subjects. Similarly, Yanagibori et al. [38] report that insulin $A U C$ during an oral glucose tolerance test was increased by $16.6 \%$ in 10 men and $74.9 \%$ in 7 women following 3 days of bed rest. The effect sizes from these nonrandomized interventions ranged from 0.34 to 3.3.

Although the majority of studies $(9 / 12)$ examining insulin sensitivity had no control group, the effect sizes of the sedentary behaviour interventions were consistently moderate to large. The results were also consistent, with 10 of 12 published studies reporting a reduction in insulin sensitivity in at least one subgroup of participants. Thus, we conclude that acute bouts of uninterrupted sedentary behaviour are likely to result in a moderate-to-large reduction in insulin sensitivity and that the available evidence is of moderate quality.

3.8. Glucose Tolerance Tests. Two randomized crossover studies $(n=32)[39,42]$ examined the impact of $2[42]$ 
and 5 hours [39] of prolonged sitting on glucose AUC in response to a standard meal, while seven nonrandomized interventions $(n=87)[23,24,27-29,38,41]$ examined the impact of uninterrupted sedentary behaviour lasting 3 $[24,38], 6[23]$, and $7[27,28,41]$ days on measures of glucose tolerance.

Both of the randomized crossover studies reported that uninterrupted sitting resulted in significant increases in glucose AUC in response to a standardized meal. Nygaard et al. reported that 2 hours of sitting resulted in a $45 \%$ increase in the glucose response to a standard meal in a group of 13 elderly women, in comparison to a combination of sitting and walking at a self-selected "very light" intensity [42]. Similarly, Dunstan et al, reported that the glucose AUC following a test meal was $33 \%$ higher following 5 hours of prolonged sitting, in comparison to 5 hours of sitting which was broken up with periodic light-intensity walk breaks [39].

Five of seven nonrandomized studies reported significant reductions in glucose tolerance in at least some participants, ranging from 7.8 to $30 \%$. For example, Smorawinski et al. [24] reported that glucose AUC during oral glucose tolerance tests was $30 \%$ higher following three days of bed rest in inactive young men, although there were no change in endurance- or strength-trained athletes. Yanagibori et al. [38] observed significant $7.8 \%$ reductions in oral glucose tolerance in women, but not men, following 3 days of bed rest. The effect sizes in these studies ranged from -0.03 to 1.4 and were in the moderate or high range for all but one study.

The available evidence suggests that acute bouts of uninterrupted sedentary behaviour may result in moderateto-large reductions in oral glucose tolerance. Given the relatively consistent findings and the strong evidence from randomized crossover studies, we conclude that the evidence linking acute sedentary behaviour with reductions in glucose tolerance is of moderate quality.

3,9. Quality Asessment, Downs and Black scores assessing the risk of bias for individual studies are presented in Table 1. The average score was $21.4 \pm 2.3$, out of a maximum of 32 . The three randomized crossover studies had the highest quality $(25.0 \pm 1.7)$, followed by the randomized controlled trials $(22.5 \pm 2.1)$ and the nonrandomized interventions $(20.8 \pm 1.9)$. The overall quality of evidence related to each outcome is presented in Table 2.

\section{Discussion}

Based on our systematic review of data from 25 independent interventions, we found moderate quality evidence suggesting that acute bouts of uninterrupted sedentary behaviour lasting 2 hours to 7 days result in rapid and deleterious changes in triglyceride levels, insulin sensitivity, and glucose tolerance. We also found very low-quality evidence that it results in changes in fasting glucose, fasting insulin, and HDL- or LDL-cholesterol.

The findings of the current paper have important public health implications. Recent estimates suggest that on average North American adults and children spend $7-10$ hours per day-more than half their waking hours-engaging in sedentary behaviour [43-46]. This suggests that many individuals likely spend several consecutive hours sitting down on a regular basis, which is not dissimilar to the protocol employed by 3 randomized crossover studies in this paper that resulted in significant reductions in insulin sensitivity and glucose tolerance $[11,39,42]$. Individuals who perform long bouts of uninterrupted sedentary behaviour on a regular basis may therefore be exposing themselves to higher levels of circulating triglycerides, as well as reduced insulin sensitivity and glucose tolerance, which may help to explain the prospective associations between sedentary behaviour and chronic disease morbidity and mortality [3-5].

Research in animal models suggests mechanisms that may explain our observation of consistent changes in both insulin sensitivity and plasma triglyceride levels in response to uninterrupted sedentary behaviour. Bey and Hamilton reported that just 18 hours of hindlimb unloading results in near total cessation of lipoprotein lipase activity and roughly $75 \%$ reduction in triglyceride uptake in rat skeletal muscle [12]. Similarly, it is also well established that skeletal muscle denervation results in rapid changes in glucose transport protein expression and reductions in insulin sensitivity $[13,14]$. These findings suggest that rapid and deleterious changes in skeletal muscle metabolic function may underlie the relationship between sedentary behaviour, triglyceride levels, and insulin sensitivity observed in the present review.

4.1. Strengths and Limitarions. The major strength of this paper is its rigorous systematic methodology. The search strategy was developed in consultation with a research librarian with expertise in search creation, and the screening process included two independent reviewers who came to consensus on all included studies. Strength of evidence was assessed using GRADE in order to increase the transparency of the grading process. Finally, the paper was prospectively registered with PROSPERO.

The limitations of this paper relate primarily to the quality of evidence that is presently available. Of 25 independent interventions identified by this paper, only 6 employed a randomized design. Further, although fasting glucose, glucose tolerance, insulin, and insulin sensitivity have each been examined by 9 or more investigations, lipid levels have received little attention by comparison. Given the small number of studies and the low quality of evidence currently available for these outcomes, it is difficult to determine their relationship with sedentary behaviour with any certainty.

There has also been a large amount of heterogeneity in the modality of sedentary behaviour ( $\mathrm{e} . \mathrm{g}$, sitting versus bed rest) and in the way that outcome measures are calculated, which precluded the use of meta-analyses in the present paper. Only 5 studies identified by the current paper examined a modality of sedentary behaviour other than bed rest. The modality of sedentary behaviour which is most common in daily life is undoubtedly sitting, yet the acute impact of sitting has only been examined in four interventions. In contrast the metabolic impact of bed rest has received far more attention in the published literature [16], despite the 
fact that probenged periods of bed rest are uncommon in day-bo-day life, Given that it is unclear whether sitting and bed rest have a similar impact on markers of cardiometabolic risk, it is important that future studies focus on the impact of sitting vo determine whether it has an impoct which is similar to that of bed rest.

The sample size of most interventions identified by this paper was cuint small, atsd the vast maipeiry of stodies were performed in physically fit, bealthy young adult males bet. ween the apes of 20 and 30 . We colly identified two interventions focused on isdividuals above the aze of $50[39,42]$, or those with ekvated body weight $[22,39]$ and we were not able to identify amy interventions focesing on pediatric populations. Further, feamales made up just $16 \%$ of the participants in the identified interventions, which makes it undear whether the relationdhips obserwal in the current paper will generalize to females of any age.

It is also worth noting that, at present, it is difficult to differemtiate the impoct of sedencury behaviour per se froen that of a positive energy balance. If energy intake is main: tained at an individual's habitual leved, it can be assumed that an imposed bout of sedentary behwiour is likely to result in positive energr balance. Howerers, to our knowlodge only one intervention [11] has attempted to sparate the impact of an acute bout of sedentary betworiour from thas of acute positive energr balance. Interestingly. Stephens et al report that reducing energy intake to match energr expenditure during a bout of probonzed sedentary behaviour reduced the deleterions impact on insulin sensitivity by ronghly $50 \%$ [11]. Further, no studies idemtified in the current poper reported adjusting results for baseline physical activity, fitness or dict. Future mork should invertigate these issues further, in oeder wo determine the relative coentributions of sedentary behariour and positive energy balance to changes in candinmetabolic rivk factors.

To dant celly three studies hawe examined the impuct of uninterrupted sedentary behwiour lasting less than 1 day on markers of metabolic risk. Given that health individuals rarely spend 24 hours engaging in unincerrupued sedemary behaviour, it is important that future studies imvestigate whether sboter bouts of sedentary behaviour also hme a mexsarable impact on metabolic health. Future work should also investizate the acule impoct of sedentary behaviour on nontraditional markers of cardiometabolic risk including adipokines and markers of inflammation. Finallk, none of the studies identified in the current paper esamined whether these deleverious changes in risk markers persistod once participants returned to free living conditions. Thus, it is undear whether the changes observed in the revieured studies endure for seretal dars following the cessation of sedemary behaviour, or whether they are rapodly resolved. Assessing the clinical significanoe of these changes will be difficult until their time-course has been more carefully examined.

\section{Condusions}

This study demonstrates that, af present, there is moderate quality evidence that acute bouts of uninterrupted sedemary behaviour result in significant and deleterious changes in insulin sensitivitk, glucose tolerance, and plasma trielyocride levek. There is currently very low-quality evidence linking uninterrupied sedentary behaviour with changes in circulating insulin, glucose, and HDL- and LDL-dwelesterol leveds. There is no evidence that acute bouts of uninternupeed sed. entary behariour prowide any positive changs in markers of cardicmetabolic risk. Horwever, the majority of studie identified by this paper focused on healthy poung men, and it is therefore undear whether these results will generalixe to temales of to other age groupse These findinges sangest that uninterrupted bouts of sedentary behaviour should be avoided in order to prewent transient increases in metabolic risk.

\author{
Abbreviations \\ HDL-cholesterol: High-density lipoprotein cholesterol \\ LDL-cholesterolt Low-density lipoprotein cholesterol \\ MFT: Metabolic equivalent \\ HOMA: Homeostasis model of assessment \\ QUICKI: Quantitative insulin sensitivity sheck \\ AUC: Area-under-the-curve.
}

\section{Funding}

T. J. Saunders and R. Larouche are supported by Doctoral Research Aoards from the Canadian Institutes of Health Besearch and Excellence Scholarahipo from the Uniezrsity of Ottarwa. T. I. Saunders is also supported by a Doctoral Besearch Awand from the Canodian Disbetes Asoociation.

\section{Conflict of Interests}

The authors report no contlict of inderens.

\section{Authers' Centribution}

T. I. Saunders, R. Larouche, R. C. Colley, and M. S. Tremblay developed the surt rationale and criteria for inclusion asd esclusion. T. J. Saunders developed and escoubed the literature search. T. I Saunders and R. Lamuche screened all potentially rekvant articles. T. I. Saunders estracted data from included studies and wrote the first draft of the paper. R. Larouche checked data extraction, then reviewed, and dited the puper with R. C. Colley and M. S. Trumblen.

\section{Acknowledgments}

The authoes are graseful to Margaret Sampoion (Childrens Hospital of Eastern Ontario Research Indtitute) for her belp in developing the sarch strategy and to their kef informants for their help identifying relevant imorveations. 


\section{References}

[1] E. B. Hu, T. Y. Li, G. A. Colditz, W. C. Willett, and J. E. Manson, "Television watching and other sedentary behaviors in relation to risk of obesity and type 2 diabetes mellitus in women," Jourmal of the American Medical Association, woL. 289, no. 14. pp. 1785-1791, 2003.

[2] I. P. Rey-López, G. Vicente-Rodriguez, M. Biosca, and L. A. Moreno, "Sedentary bebaviour and obesity development in children and adolescents," Nutrition, Metubolism and Cardiovascular Diseases, vol. 18, no. 3, pp. 242-251, 2008.

[3] K. I. Proper, A. S. Singh, W. Van Mechelen, and M. J. M. Chinapus, "Sedentary behaviors and health outcomes among adults: a systematic review of prospective studies," American Journal of Preventive Medicine, vol, 40, no, 2, pp, 174-182. 2011.

[4] P. T. Katzmarzyk, T. S. Church, C. L. Craig, and C. Bouchard, "Sitting time and mortality from all causes, cardiovescular dis ease, and cancer," Medicine and Science in Sports and Exarcise. vol. 41, no. 5, pp. 998-1005, 2009.

[5] E. Stamatakis, M. Hamer, and D. W. Dunstan, "Screen-based entertainment time, all-cause mortality, and cardiovascular events: population-based study with ongoing mortality and hospital events follow-up," Journal of the American Colliege of Cardiology, vol. 57, no. 3, pp. 292-299, 2011.

[6] T. Y. Warren, V. Barry, S. P. Hooker, X. Sui, T. S, Church, and S N. Blair, "Sedentary behaviors increase risk of cardiovascular disease mortality in men," Medisine and Scrience in Sports and Exercise, vol. 42, no, 5, pp. 879-885, 2010.

[7] B. M. Lynch, "Sedentary behavior and cancer: a systematic review of the literature and proposed biological mechanisms," Cancer Fpidemiology Eiomarkers and Prevention, wol. 19, no. 11, pp. 2691-2709, 2010.

[8] M. T. Hamilton, D. G. Hamilton, and T. W. Zderic, "Role of lowr energy expenditure and sitting in obesity, metabolic s.n drome, type 2 diabetes, and cardiovascular disease," Diabetes. vol. 56, no. 11, pp. 2655-2667, 2007.

[9] M.S. Tremblay, R. C. Colley, T. I. Saunders, G. N. Healy and N. Owen, "Physiological and health implications of a sedentary lifestyle, Applied Physiology, Nurrition and Metabolism, wol 35, no. 6, pp. 725-740, 2010.

[10] N. M. Hamburg, C. I. McMackin, A. L Huang et al., "Physical inactivity rapidly induces insulin resistance and microvascular dysfunction in bealthy volunteers," Arteriosderosis, Thrombosis, and Vascular Biology, wol. 27, no. 12, pp. 2650-2656, 2007.

[11] B. R. Stephens, K. Granados, T. W. Zderic, M. T. Hamilton. and B. Braun, "Effects of 1 day of inactivity on insulin action in healthy men and women: interaction with energy intake," Metabalism, vol. 60, no. 7, pp. 941-949, 2011.

[12] L. Bey and M. T. Hamilton, "Suppression of skeletal muscle lipogrotein lipase activity during phrsical inactivity: a molecular reason to maintain daily low-intensity activity," Journal of Physiology, vol. 551, no, 2, pp. 673-682, 2003.

[13] J. . Wilkes and A. Bonen, "Reduced insulin-stimulated glucose transport in denervated muscle is associated with impaired Akt- $\alpha$ activation," American Journal of Physiology, wol. 279, no 4. Pp. E912-E919, 2000.

[14] L. Coderre, M. M. Monfar, K. S. Chen et al., "Alteration in the expression of GLUT- 1 and GL.UT-4 protein and messenger RNA levels in denervated rat muscles," Endocrinalogy, vol. 131 . no. 4, pp. 182I-1825, 1992.

[15] M. T. Hamilton, G. N. Healy, D. W. Dunstan et al., "Too little exercise and too much sitting inactivity physiology and the need for new recommendations on sedentary behavior," Current Candionaccular Risk Reports, wol. 2, pp, 292-298, 2008,

[16] A. Bergouignan, F. Rudwill, C. Simon, and S. Blanc, "Physical inactivity as the culprit of metabolic inflexibility; evidence from bed-rest studies," Journal of Applied Physiology, vol. 111, pp. 1201-1210, 2011.

[17] S. H. Downs and N. Black, "The feasibility of creating a checklist for the assessment of the methodological quality both of randomised and non-randomised studies of bealth care interventions," Journal of Epidemiology and Community Health, vol. 52, no, 6, pp, 377-384, 1998.

[18] G. H. Guyatt, A. D. Oxman, G. E. Vist, R. Kunz, Y. Falck-Ytter, and H. I. Schünemann, "GRADE: what is "quality of evidence" and why is it important to clinicians?" British Modical Journal, vol. 336, no, 7651, pp. 995-998, 2008.

[19] E. A. Richter, B, Kiens, M. Mizuno, and S. Strange, "Insulin action in human thighs after one-legged immobilization," Journal of Applied Physialogy, vol. 67, no. 1, pp. 19-23, 1989.

(20) V. E Katkov, V. V. Chestukhin, and L I. Shefter, "Short-term immobilization of healthy men: right ventricular function and metabolism during graded exercise, Cor ef Vasu, vol. 21, no. 1, pp. 61-70, 1979.

(21) A. C. Alibegovic, M. P. Sonne, L Hojbjerre et al., "The T-allele of TCF7L.2 rs7903146 associates with a reduced compensation of insulin secretion for insulin resistance induced by 9 days of bed rest," Diabetes, vol. 59, no. 4, pp. 836-843, 2010.

(22) E. Duran-Valdez, D. G. De Serna, S. Schneider, F. Amorim, M. Burge, and D. S. Schade, "Metaholic effects of 2 days of strict bed rest," Endocrine Practice, vol. 14, no. 5, pp, 564-569, 2008.

[23] S. Blanc, S. Normand, C. Pachiaudi, J, O. Fortrat, M. Laville, and $\mathrm{C}$. Gharib, "Fuel homeostasis during phrsical inactivity induced by bed rest," Journal of Clinionl Endocrinology and Metabolism, vol. 85, no, 6, pp, 2223-2233, 2000.

[24] I. Smormsióski, H. Kaciuba-Ułcilko, K. Nazar et al, "Effects of three-day bed rest on metabolic, hormonal and circulatory responses to an oral glucose load in endurance or strength trained athletes and untrained subjects," Journal of Physiology find Pharmacology, vol. 51, no. 2, pp. 279-289, 2000.

[25] P. Barbe, J. Galitzky, C. Thalamas et al., "Increase in epinephrine-induced responsiveness during microgravity simulated by head-down bed rest in humans," Journal of Applind Physialogy, vol. 87, no. 5, pp. 1614-1620, 1999.

[26] I. Nygren, A. Thorell, S. Efendic, K. S. Nair, and O Liungevist, "Site of insulin resistance after surgery: the contribution of hypocaloric nutrition and bed rest," Clinical Science, wol. 93. no. 2, pp, 137-146, 1997,

[27] K. J. Mikines, F. Dela, B. Tronier, and H. Galbo, "Effect of 7 dars of bed rest on dose-response relation between plasma glucose and insulin secretion," American Journal of Physiology, vol, 257, no, 1, pp. E43-E48, 1989.

[28] C. A. Stuart, R. E. Shangraw, M. L. Prince, E. I. Peters, and R. R Wolfe, "Bed-rest-induced insulin resistance occurs primarily in muscle," Mctabolism, vol. 37, no. 8, pp. 802-806, 1988.

(29) R. L. Lipman, P. Raskin, T. Love, I. Triebwasser, F. R. Lecocq, and I. J. Schnure, "Glucose intolerance during decreased physical activity in man," Dhabetes, vol. 21, no. 2, Pp. 101-107, 1972.

[30] C. Moro, F. Pillard, L. De Glisezinski et al., "Atrial natriuretic peptide contribution to lipid mobilization and utilization during head-down bed rest in humans," American Journal of Physiology, vol. 293, no. 2, pp. R612-R617, 2007.

[31] A. C. Alibegovic, L. Heibjerre, M. P. Sonne et aL, "Impact of 9 days of bed rest on bepatic and peripheral insulin action, insulin secretion, and whole-body lipolysis in healthy young 
male offspring of patients with type 2 diabetes," Diabetes, wol. 58, no. 12, pp. 2749-2756, 2009.

[32] D. Kanikowska, M. Sato, S, lwase et al. "Leptin and ghrelin levels in humans during physical inactivity induced by headdown bed rest," Aviation Space and Environmental Medicine, vol. 81, no. 4, pp. 383-386, 2010.

[33] Y. G. Zorbas, Y. L Yarullin, S. D. Denogradov and Y. B. Afonin, "Plasma volume and biochemical changes in athletes during bed rest chronic hyperhydration," Acta Astronautick, vol. 45 , no. 12, pp. 747-754, 1999.

[34] N. M. Navasiolava, F. Dignat-George, F. Sabatier et al., "Enforced physical inactivity increases endothelial microparticle levels in healthy volunteers" American lournal of Physioogy, vol. 299, na 2, pp. H248-H256, 2010.

[35] C. B. Dolkas and J. E. Greenleaf, "Insulin and glucose responses during bed rest with isotonic and isometric exercise," Journal of Applied Physioloxy Respiratory Environmental and Exercise Physiology, vol, 43, no, 6, pp. 1033-1038, 1977.

[36] L. Ksinantova, I. Koska, R. Kvetnansky, M. Marko, D. Hamar, and M. Vigas, "Effect of simulated microgravity on endocrine response to insulin-induced hypoglycemia in physically fit men, Hormone and Metabolic Research, wol. 34, no, 3, pp. 155$159,2002$.

[37] Y. Y. Yaroshenko, Y. G. Zorban, N. K. Kuznetsov, A. G. Kakurin, V. K. Popov, and V. L. Yazulin, "Changes in thyroid hormones and lipids in endurance trained volunteers during acute and rigorous bed rest conditions," Wiener Klinische Wodienschrift, vol. 110, no, 6, pp. 225-231, 1998.

[38] R. Yanagibori, Y. Suzuki, K. Kawakubo et al. "The effects of 20 days bed rest on serum lipids and lipoprotein concentrations in healthy young subjects," Jourmal of Gravitational Physialogy, vol. 4, no. 1, pp. \$82-590, 1997.

[39] D. Dunstan, B. A. Kingwell, R Larsen et al., "Breaking up prolonged sitting reduces postprandial glucose and insulin responses," Diabetes Care, vol. 35, no. 5, pp. 976-983, 2012.

[40] K. I. Mikines, E. A. Richter, E. Dela, and H. Galbo, "Seven days of bed rest decrease insulin action on glucose uptake in leg and whole body, Journal of Applied Physiology, wol. 70, no. 3, pp. 1245-1254, 1991.

[41] K. Kiilerich, S. Ringholm, and R. S. Bienso, "Exercise-induced pyruvate debydrogenase activation is not affected by 7 days of bed rest, Journal of Applied Physialogy, wol. 111, pp. 751-757, 2011.

[42] H. Nygaard, S. E. Tomten, and A. T. Hestmark, "Slow postmeal walking reduces postprandial glycemia in middle-aged women," Applied Physialogy, Nutrition and Metabolism, wol. 34, no, 6, pp, 1087-1092, 2009.

[43] R. C. Colley, D. Garriguet, 1. Janssen, C. L. Craig, J. Clarke, and M. S. Tremblay, "Physical activity of Canadian adults: accelerometer results from the 2007 to 2009 Canadian Health Measures Survey," Healeh Reports, vol. 22, no. 1, pp. 7-14, 2011.

[44] R. C. Colley, D. Garriguet, I. Janssen, C. L. Craig, I. Clarke, and M. S. Tremblay, "Physical activity of Canadian children and vouth: axcelerometer results from the 2007 to 2009 Canadian Health Measures Survey, Health Reports, vol. 22, no 1, pp. 15-23, 2011.

[45] V. Carson and I. lanssen, "Volume, patterns, and types of sedentary behavior and cardio-metabolic hezlth in children and adolescents: a cross-sectional study," BMC Public Health, vol. 11, article 274, 2011.

[46] C. E. Matthews, K. Y. Chen, P. S. Freedron et al, "Amount of time spent in sedentary behaviors in the United States, 20032004, American foumal of Epidemialogy, vol. 167, no, 7, pp $875-881,2008$.
(47) S. Blanc, S. Normand, C. Pachiaudi, M. Duvareille, and C. Gharib, "Leptin responses to physical inactivity induced by simulated weightlessness," American fourmal of Physiology, vol. 279, no. 3, pp. R891-R898, 2000.

[48] J. Smorawinski, H. Kaciuba-Uscilloo, K. Nazar, E. Kaminska, P. Korseun, and J. E. Greenleaf, "Comparison of changes in glucose tolerance and insulin secretion induced by three-day bed rest in sedentary subjects and endurance or strength trained athletes," Journal of Gravitational Physiology, vol. 5, no, 1, pp. P103-P104, 1998.

(49] I. Smorawinski, P. Kubala, H. Kaciuba-Uocisko, K. Nazar, E. Titow-Stupnicka, and J. E. Greenkeaf, "Effects of three day bed-rest on circulatory, metabolic and hormonal responses to oral glucose load in endurance trained athletes and untrained subjects," Journal of Gravitational Physialogy, wol. 3, no. 2, pp. 44-45, 1996. 


\title{
Sedentary Behaviour, Visceral Fat Accumulation and Cardiometabolic Risk in Adults: A 6-Year Longitudinal Study from the Quebec Family Study
}

Travis J. Saunders ${ }^{1,2 *}$, Mark S. Tremblay ${ }^{1,2,3}$, Jean-Pierre Després ${ }^{4,5}$, Claude Bouchard ${ }^{6}$, Angelo Tremblays, Jean-Philippe Chaput ${ }^{1,2,3}$

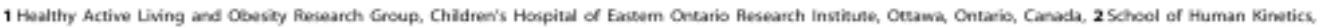

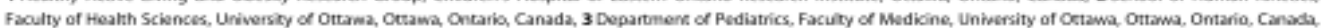

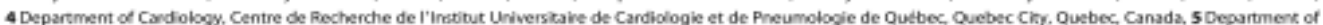
Nnesiblogy. Faculy of Medicine, Lawal University, Quebec City, Ouebec, Canada, 6 Haman Genomics Laboratory. Pennington Bomedical Research Center, Baton Aouge, Lousians, United States of America

\begin{abstract}
Background: Sedentary behaviour has recently emerged as a unique risk factor for chronic disease morbidity and mortality. One factor that may explain this relationship is visceral adiposity, which is prospectively associated with increased cardiometabolic risk and mortality. The objective of the present study was to determine whether sedentary behaviour was associated with increased accumulation of visceral fat or other deleterious changes in cardiometabolic risk over a 6 -year follow-up period among adult participants in the Quebec Family Study.

Methods: The current study included 123 men and 153 women between the ages of 18 and 65 . Total sedentary time and physical activity were assessed by self-report questionnaire. Cross-sectional areas of visceral and subcutaneous abdominal adipose tissue were assessed using computed tomography. Cardiometabolic biomarkers including fasting insulin, glucose, blood Fipids, HOMA-Insulin Resistance, and oral glucose tolerance were also measured. All variables of interest were collected at both baseline and follow-up.

Results: After adjustment for age, sex, baseline BMI, physical activity, energy intake, smoking, education, income and menopausal status, baselline sedentary behaviour was not associated with changes in visceral adiposity or any other marker of cardiometabolic risk. In the longitudinal model which adjusted for all studied covariates, every 15 -minute increase in sedentary behaviour from baseline to follow-up was associated with a $0.13 \mathrm{~cm}$ increase in waist circumference ( $95 \%$ $\mathrm{Cl}=0.02,0.25$ ). However, there was no association between changes in sedentary behaviour and changes in visceral adiposity or other markers of cardiometabolic risk.

Conclusion: These results suggest that neither baseline sedentary behaviour nor changes in sedentary behaviour are associated with longitudinal changes in visceral adiposity in adult men and women. With the exception of waist circumference, the present study did not find evidence of a relationship between sedentary behaviour and any marker of cardiometabolic risk in this population.

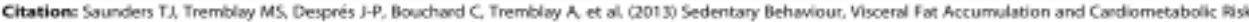

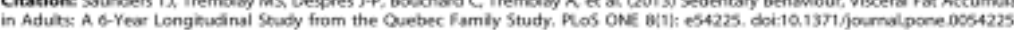

Editon Kaberi Daspupta, McGall University, Canado

Received August 1, 2012; Accepted December 11, 2012; Published January 9, 2013

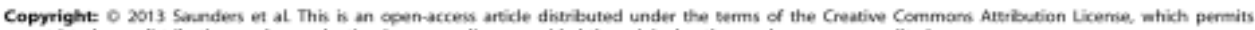
unestrictod use, datribution and reproduction in any modiam, providod the original author and source ane ovdited.

Funding: The Quebec family Study was supoorted over the years by muliple grants from the Medical Research Council of Canoda and the Canadian Institubes

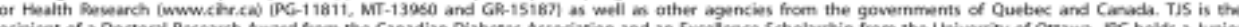

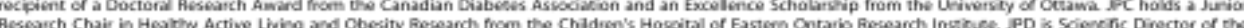

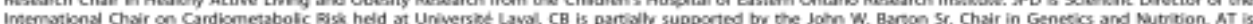
partisty funded by the Canada Plesearch Char in Emwinonment and Energy Balance. The finders had no nole in study desion data colection and anatysis, decision to publath or preparation of the manuscript

Competieg Interests! The authors have declared that no compesing imterests exist

- Emal:
\end{abstract}

Introduction

Sedentary behaviour (e,g, sitring, reclining) has recently emerged as a unique risk factor for chronic disease $[1,2]$ and is consistently associated with increased risk of both obesity ani mortality [3-6]. Excess sedentary time has also been associated with increased accumulation of central adiposiry and ocher markers of cardiometaholic risk [7, g]. For exampls, Wijndacte and colleagues reported that increases in television (TV) viewing during a 5-year follow-up period were associated with significant increases in waist circumference in both men and women, and increases in blood pressure and dustered cardiometabolic risk among worren [8]. 
One factor that may link sedentary behaviour with increased monhidity and mortality is the accumulation of visoeral adipose tissue, which is prospectively associated with mortality and increaed cardiometabolic risk [9-11]. Despite the hypothesized link between high levels of sedentary behaviour and both obesity and central adiposity, the association between sedentary behaviou and the accumulation of visceral adipose tisse remains largely unexamined. To our knowkder, only one cross-sectional study has examined this question, and reported no association between sedentary behaviour and visecral fat kevels in physically inactive adults [12].

A longitudinal study of the association between sedentary behaviour and the accumulation of visceral fat could therefore make an important contribution to our understanding of the relationship between sedentary behaviour and chronic disease morbidity and mortality. The objective of the present study was to determine whether sedentary behaviour was associated with increased accumulation of visceral fat or other deleterious changes in cardiometabolic risk among adult participants in the Quebec Family Study.

\section{Materials and Methods}

\section{Ethics Statement}

All participants provided written informed coesent to participate in the study. The project folloned guidelines of the Medical Rexarch Councal of Canada, and was approsed by the Medical Ethics Committee of Laval University.

\section{Subjects}

The Quebec Family Study was initiated at Laval University in 1978. The primary gosal of this progect was to investizate the role of genetics in the development of obesity and related cardiovascular risk factors. A total of 1630 individuals from 375 families were recruited and assesed in Phase I of the stuxly (1978 to 1981). I this initial phase recruitment was conducted irrespective of body weight, resulting in a cohort with body mass index (BMI), ranging

Table 1. Baseline subject characteristics across tertiles of baseline sedentary behaviour in males.

\begin{tabular}{|c|c|c|c|c|}
\hline & n & Low & Medium & High \\
\hline Age (years) & $39 / 33 / 51$ & 39 [15] & 39 [13] & $30(16)$ \\
\hline Baseine sedentary time iminiday) & $39 / 30 / 51$ & $30562 \%$ & 472500 & $607|\operatorname{sg}|$ \\
\hline MVPA imindayl & $39 / 32 / 51$ & 501601 & $41[47]$ & $25(36)$ \\
\hline BMI $\left(\mathrm{kg} / \mathrm{m}^{2}\right)$ & $39 / 33 / 51$ & $25.8|4.4\rangle$ & $26.5(4.6)$ & 26.4 (5.5) \\
\hline Waist chesmference $1 \mathrm{~km}$ ) & $39 / 33 / 51$ & 89.4 [12.0) & $913[12.1]$ & 909 (15.9) \\
\hline Body fat (⿻) & $37 / 31 / 46$ & $21.3|7.5\rangle\rangle$ & $225(7.4)$ & $215(8.6)$ \\
\hline Visceral AT $\left(\mathrm{cm}^{2}\right\}$ & $29 / 29 / 36$ & $112.5(61.1)$ & $124.9(67.00)$ & 1147 (85.5) \\
\hline Subculanecus AT $\left(\mathrm{cm}^{2}\right)$ & $20 / 22 / 36$ & $200.2(13000)$ & $235.5\langle 132.1\rangle$ & $191.6\{120.6\}$ \\
\hline Total abdominsl AT $\left(\mathrm{cm}^{2}\right)$ & $29 / 29 / 36$ & 320.7 (1769) & $3604(189.5)$ & $306.3(197 / A)$ \\
\hline Fasting glucose ommolu & $39 / 33 / 51$ & 5.00 [0.59) & 4.93 [0.57| & $4.97(0.52)$ \\
\hline HOMA $\mathrm{R}$ index & $28 / 30 / 39$ & $2.32(1.72)$ & $2.56[2.77]$ & $2.87(2.46)$ \\
\hline Ghacose ALC Immoll & $30 / 2 \mathrm{x} / 37$ & $1224(244)$ & $1126[197)$ & 1143 (253) \\
\hline Insulin AUC Ipmolu & $28 / 28 / 36$ & 692496076701 & $66615(43996$ & $732000(50413)$ \\
\hline Total cholesterol (mmahu & $39 / 22 / 50$ & 4.95 [0.86] & 5.1510 .901 & $4.89(1.02)$ \\
\hline HDL-chabusterd (mmol/L) & $39 / 33 / 50$ & $1.16 \mid 0.300$ & $1.13|0.22|$ & $1.08(0.25)$ \\
\hline ID-cholesterol Immollu & $36 / 2 / 50$ & 3.17 (a.n] & 3.25 |ar3| & 313 inas \\
\hline Triglycerides (mmollu) & $39 / 33 / 90$ & 1.49 (a.90) & 1.77 [1.18| & $1.56(0.76)$ \\
\hline Energy incake (had/day) & $39 / 32 / 51$ & $2910(596)$ & $2714(715)$ & 2672 (8003) \\
\hline \multicolumn{5}{|c|}{ Total family income in Canadian dollars in IND0 } \\
\hline$<70,0005$ & & $0(0) 1$ & 0,01 & 0,06 \\
\hline $10,000-29,0005$ & & 12.61 & 0001 & 0,000 \\
\hline $30,000-8,0005$ & & 13 [342] & $13[37.1\}$ & $8\{170\}$ \\
\hline $50,000-69,0006$ & & $13[34.2]$ & $9(25 \pi)$ & $13(27)$ \\
\hline $70,0005+$ & & 11 (28.95) & $13|37.1|$ & $26(55.3)$ \\
\hline \multicolumn{5}{|l|}{ Education loved } \\
\hline High School & & 20 [48:8] & $902 . n$ & $10(21, n$ \\
\hline Colkge" & & $14[342]$ & $12[34.3 \mid$ & $21(45, \pi)$ \\
\hline Uniwersity & & $7(17.1)$ & 1410001 & $15(32.6)$ \\
\hline
\end{tabular}

Dua are expresied as mean (SD) unlers othermie specifiod.

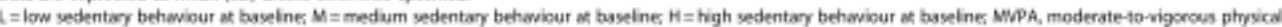
activity, BMI, body mass index AT, adpose thsue: HOMh- R homeostass modet assessment of ingulh reskstance: AuC, area under-the-curve

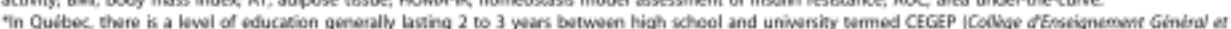

Professionnel. an acrorym that does not heve ary trandation in finglish

Professioninel an acrorym that does nof i 
Table 2. Baseline subject characteristics across tertiles of baseline sedentary behaviour in females.

\begin{tabular}{|c|c|c|c|c|}
\hline & n (L/M/M0 & Low & Medium & High \\
\hline Age (yoars? & $54 / 58 / 41$ & $41[12]$ & $42[15]$ & $32(134)$ \\
\hline Baveline sedentary time (min/day) & $54 / 54 / 41$ & 305000 & $463(41)$ & 6201601 \\
\hline MUPA iminidgyl & $53 / 57 / 30$ & $21|26|$ & $10[15]$ & $14(16)$ \\
\hline sMI $\left(\mathrm{kg}^{2} \mathrm{~m}^{2}\right)$ & $54 / 58 / 41$ & $25.216 .0 \%$ & $253(6.3)$ & $245(4.7)$ \\
\hline Waást creumference $(\mathrm{cm})$ & $54 / 58 / 41$ & 73.9 [14.5] & 79.0 [13.8] & 76.8011 .9 \\
\hline Body fat (WeI & $45 / 50 / 40$ & 30.8 [10.1\} & 31.219 .00 & $20.4(183)$ \\
\hline Msceral AT $\left(\mathrm{cm}^{2}\right)$ & $41 / 42 / 29$ & 87.7 161.8] & $101.2(55.9)$ & $64.4(44,0)$ \\
\hline Subculanecous AT $\left[\mathrm{cm}^{2}\right)$ & $41 / 42 / 29$ & 282.8 (154.8) & 312.5 (16.2.61 & $268.1\{147.4\}$ \\
\hline Total abdomind AT (com') & $41 / 42 / 29$ & 370.5002 .21 & 413.7000 .91 & $332.5\{180.61$ \\
\hline Fasting giucose ímmolul & $54 / 50 / 40$ & 4.06 \{a.51] & $4.81 \mid 0.521$ & 40910.401 \\
\hline HOMW-F Index & $44 / 45 / 32$ & 2.29 [1.96] & 2.2312 .001 & $1.94(1.27)$ \\
\hline Glucose ALC immol/L) & $40 / 41 / 29$ & $10751200)$ & $1162[257)$ & 1101 (156) \\
\hline Insulin ALX Ipmolu & $39 / 41 / 29$ & $66290(46-463)$ & $79134\langle 74763\rangle$ & $6100714269 \%$ \\
\hline Total chokestenol (mmollu & $5457 / 39$ & $5.10\{1.01\}$ & $5.16[1.03]$ & 5.0112 .001 \\
\hline HDL-cholesterd (mmol/L) & $54 / 5739$ & 1.40 (10.36) & $1.37|0.32|$ & $1.32(0.37)$ \\
\hline IDC-shdenterol immollLs & $54 / 5758$ & 3,13 10.50 & 3.1810 .601 & 2.8404 .084 \\
\hline Triglycerides immolu & $54 / 57 / 39$ & 1.28 10.591 & 1.3910 .621 & $200[5.11]$ \\
\hline Energy hake (kcalday) & $54 / 56 / 41$ & $1877|381\rangle$ & 1869 [396] & $2096(434)$ \\
\hline \multicolumn{5}{|c|}{ Totad family income in Canadian dollars in (s)io } \\
\hline$<10,0005$ & & 1 (1.80) & 20.01 & $1(2,2)$ \\
\hline $10,000-29,0005$ & & $1(1.8)$ & 0,01 & 0,100 \\
\hline $30,000-49,0005$ & & 25 [45.5] & $16[29.1]$ & $6(16.7)$ \\
\hline $50,000-69,0005$ & & 13 [23.6] & 14 [25.5] & $10(27.8)$ \\
\hline $70.0005+$ & & $15|223|$ & 23 |41,月| & $19(52.8)$ \\
\hline \multicolumn{5}{|l|}{ Education kevel } \\
\hline High School & & 29 [50.9] & $30[50.91$ & $10(27.8 \%$ \\
\hline College*" & & $15|203|$ & 21135.01 & $16(43.2)$ \\
\hline University & & 13 [22.8] & $8 \leqslant 13.6)$ & $11(29.7)$ \\
\hline \multicolumn{5}{|l|}{ Menopsusal stahus } \\
\hline In menopiase & & 13 [36.1] & 15 [е6.91 & $3(30)$ \\
\hline Not in menopause & & 23 16391 & 17 [53.11 & $7(70)$ \\
\hline
\end{tabular}

Dasa are expressed as mean ISD) uniess otherwise specified.

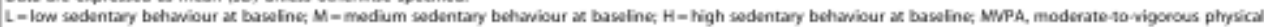

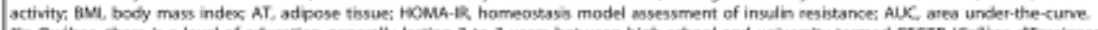

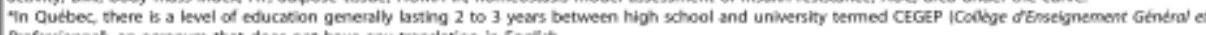

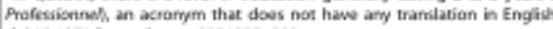

doi:10.13r1/jounstpoce 0054225 to002

from 13.8 to $64.9 \mathrm{~kg} / \mathrm{m}^{2}$. An additional 123 families with at keast I parent and 1 offspring with a BMI of 32 or highes were added to the stuxly in Phase 2 (1989-1994) and 3 (1995-2001) of the study, while also retesting 100 fannilies from Phase 1. Families were recruited through the media and were all French Canadians from the greater Qudtee City area. From the sample of 223 whits nuclear families itocaling 951 subjects imolved in Phases 1, 2, and 3) 147 men and 169 women were eligible for longitudinal analyses between Phase 2 and 3. Longitudinal analyses were not possible with Phase 1 as assessments differed at this time point from those employed in Phases 2 and 3. Additional details about the Quebec Family Study have been peeviously published [13].

Baseline in the current study correspooded to Phase 2, and the mean duration of follow-up between Phase 2 and 3 was 6.0 [SD 1.0] yearx. The following exclusion criteria were applied: (i) aged less than 18 years or greater than 64 years 13 men and 9 womes excluded: (ii) diabetic, defined as use of insulin or a hypoghycemic agent, a fasting plasma glucose level of $\geq 7.0 \mathrm{mmol} / \mathrm{L}$, or a 2 -hour postoad plasma glocose level of $\geq 11.1 \mathrm{mmol} / \mathrm{L}$ (7 men and 3 women excluded) and (iii) missing data for sedentary behaviour (4 men and 4 women excluded]. The final number of eligible participants within the longitudinal sample was 286 individuals (123 men and 153 women) (see Tables 1 and 2).

\section{Sedentary Behaviour and Physical Activity}

Sedentary bebaviour and physical activicy were estimated using a physical activity record [14]. Sulhjects had to complete a physical activity diary foe 3 days, including 2 weekdays and 1 weekend day, with each day being divided into 96 periods of 15 minutes each. Subjects were asked to code the main activity performexd during each 15-minute period using a scale from 1 to 9 , ranging from sleeping (category 1) to intense manual work (category 9). Time 
Table 3. Associations (95\% confidence interval) of sedentary behaviour and markers of adiposity at baseline.

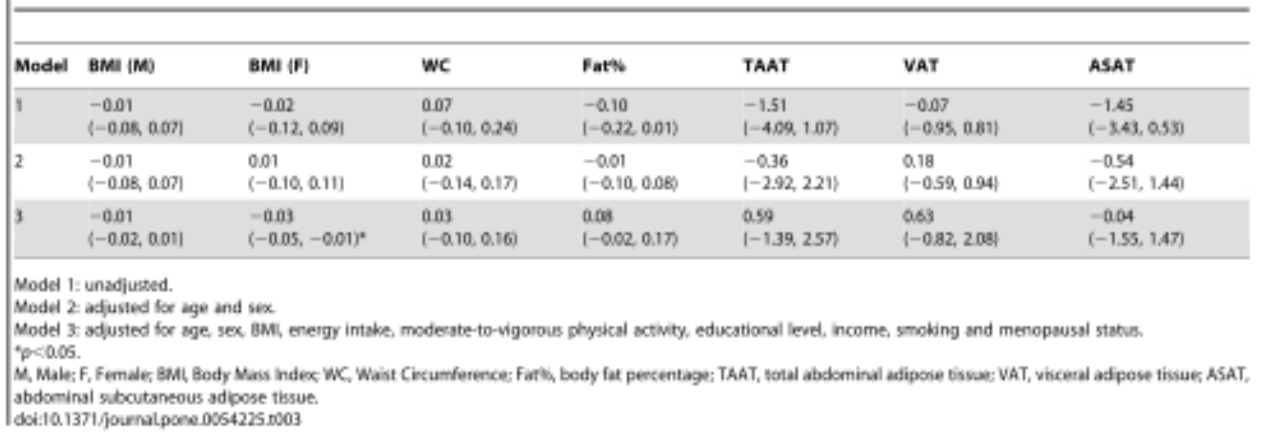

spent in categories 6 to 9 was used to cakulate moderate-tovigorous physical activity (MVPA) [14]. Sedentary behaviour was calculated as the sum of time identified as being in category 2 ("Sitting: eating, listening, writing, ete") Time spent in category 1 ("slecping, resting in bed") was not included in the sedentary behaviour categrory as sedentary behaviour refers only to waking behaviours [2]. The reliability and validity of the record have been previously reported [14]. These measurements were performed both at bascline and after 6 years.

\section{Assessment of Abdominal Fat by Computed Tomography $(C T)$}

Cros-sectional abdominal adipose tissue areas were assessed by CT using a Sicmens Somatom DRH scanner (Erlanger, Germany) as described in detail elsewhere [15]. Briefly, an abdominal scan was taken between the fourth and fifth lumbar vertebrae (L $/$ - L.5) with subjects lying in a supine position with arms stretched above the bead. The poestion of the scan was determined using a scout radiograph of the abdoenen. Total and visceral adipose tissue areas were delineated with a graph pen and then computed using an attenuation range of -190 to -30 Hounsfickd units [16]. Vixceral fat area was determined by drawing a line within the musck wall surrounding the abobominal cavity. Abdominal subcutaneous fat area was obtained by computing the difference between total and visceral adipose tissue areas.
Anthropometric and Body Composition Measurements

Height was measured to the neares $0.1 \mathrm{~cm}$ using a standard stadiometer, and body weight was measured to the nearest $0.1 \mathrm{~kg}$ using a digital panel indicator scale (Beckman Industrial Lid. Model 610/612, Scotland, UK. BMI was calculated as body weight divided by beight squared $\left(\mathrm{kg} / \mathrm{m}^{2}\right)$. Waiss circumference was measured at the line between the lower border of the last rit and the upper border of the iliac crest. All anthropoenetrix measurements were performed acoording to standardized proce dures recommended at The Airlie Conference [17]. Body density was obtained from the mean of 6 valid measurements derived from underwater wrighing [18], The helium dilution method of Meneely and Kaltreider [19] was used to determine the pulmonary residual volume before immersion in the hydrostatic tank.. Total body fat percentage was determined from body densily with the equation of Siri [20]. Body fat mass was estimated from body weight and the percentage of body fat. These measurements were performed in the same way at boch baseline and after 6 years.

\section{Cardiometabolic Risk Factors}

Total cholesterol and trighyceride concentrations were determined by use of commencial enxymatic-based methods, as described elsewbere [21]. HDL-cholesterol conocatrations wer analyzed after precipitation of apolipoprotein B-containing lipoproteins with heparin and manganese chloride [22]. Glucose concentrations were measured enzymatically and serum insulin

Table 4. Associations (95\% confidence interval) of sedentary behaviour and markers of cardiometabolic risk at baseline.

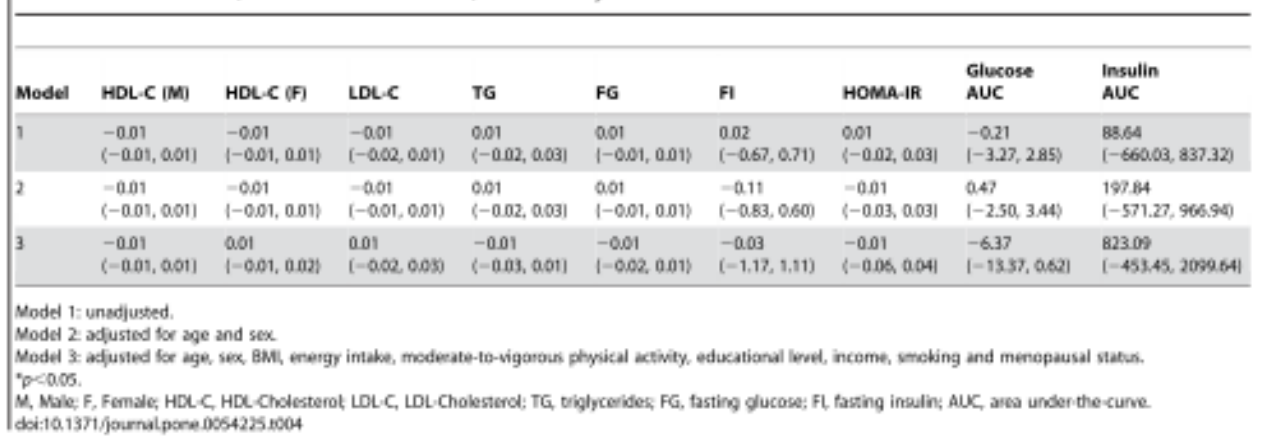


Table 5. Associations (95\% confidence interval) of baseline sedentary behaviour with 6-year change in markers of adiposity.

\begin{tabular}{|c|c|c|c|c|c|c|c|}
\hline Model & BMI (M) & BMI (क) & we & Fat 6 & TAAT & VAT & ASAT \\
\hline 1 & $\begin{array}{l}0.01 \\
(-0,02,0.05)\end{array}$ & $\begin{array}{l}-0.04 \\
{[-0.06-0.01 \%}\end{array}$ & $\begin{array}{l}-6.02 \\
1-0.09,0.05\end{array}$ & $\begin{array}{l}-0.03 \\
\{-0.06 \\
\mid 0.02\}\end{array}$ & $\begin{array}{l}-0.47 \\
\langle-157,0.63\}\end{array}$ & $\begin{array}{l}-0.23 \\
(-0.73,0201\end{array}$ & $\begin{array}{l}-0.24 \\
(-1.05,0.56\end{array}$ \\
\hline 2 & $\begin{array}{l}0.01 \\
(-0.02,0.05)\end{array}$ & $\begin{array}{l}-0.04 \\
{[-0.08,-0.01 \%}\end{array}$ & $\begin{array}{l}-0.02 \\
1-0.10,0.05\}\end{array}$ & $\begin{array}{l}-0.03 \\
\{-0.08,0.02\}\end{array}$ & $\begin{array}{l}-0.33 \\
\{-1.47, \text { a.81\} }\end{array}$ & $\begin{array}{l}-0.19 \\
(-0.70,0.33)\end{array}$ & $\begin{array}{l}-0.14 \\
(-0.98,0.70)\end{array}$ \\
\hline 3 & $\begin{array}{l}-0.02 \\
(-0.06,0.05)\end{array}$ & $\begin{array}{l}0.03 \\
1-0.05,0.11)\end{array}$ & $\begin{array}{l}-0.08 \\
\mid-0.19,0.05\}\end{array}$ & $\begin{array}{l}-0.07 \\
\{-0.15,0.02\}\end{array}$ & $\begin{array}{l}-0.69 \\
\langle-2.92,1.53|\end{array}$ & $\begin{array}{l}-0.16 \\
(-1.38,1.05)\end{array}$ & $\begin{array}{l}-0.53 \\
(-1.91,0.86\end{array}$ \\
\hline
\end{tabular}

Modet 1: unadjusted.

Nodel 2 adustied for age and sex

Model 3 adfusted for age, sex, baseline ami, energy intake, modente-to-vigorous physical activity, educationsl leved, income, smoking and menopousal status

tecio.05.

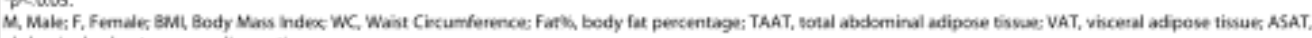
abdominal suboutaneous adpose tissue

concentrations were measured by radioimmunoassay [23]. A $75 \mathrm{~g}$ oral glucose tolerance test (OGTT) was performed in the morning after a $12 \mathrm{~h}$ fast. The total areas under the curve during the OGTT for insulin and glucose were computed from the plasma levels determined at $15 \mathrm{~min}$ intervals during the first hour following the glucose ingestion and every $30 \mathrm{~min}$ for the subsequen $3 \mathrm{~h}$, using the raperoidal method as perviously described [24]. Insulin sensitivity was estimated in the fasting state using the homesotasis model assessment for insulin resistance (HOMA-IR] [25].

\section{Energy Intake}

Diet was evaluated with a 3-day food recoed, including 2 weekdays and 1 weekend day, at baseline and year 6 . Participants were shown how to complete this record by a dietician who provided instruction about measuring the quantities of ingested foods [26]. Mean daily energy intake was estimated by a dietician using a computerized version of the Canadian Nutrient File $[27]$.

\section{Measurement of Covariates}

Several cosariates were measured via self-reported ogestionnaires. These indude age, sex, smoking habits (nonsmoker or exsmoker, light smoker [ 510 cigarettes per day], heavy smoker [ $>10$ cigarettes per dayl, highest educational level (high school, college [CEGEP for Quebec], university], total annual family income icategorized into 5 groupes ranging from $<\$ 10,000$ to $\$ 70,000$ or more) and menopausal status.

\section{Statistical Analysis}

Sample size calculations were performed to assess whether the present dataset was likely to provide sulficient power to detect significant relationship between sedentary behaviour and longitudinal changes in our primary outcome of viseeral adiposity, shoukl one exist. Assuming that the fully adjusted model would accoun for roughly $25 \%$ of the variance in changes in visceral adiposity during the f-year follow-up, and that sedentary hehaviour woukl account for at least $3 \%$ of this variance, the current dataset of $20 \%$ participants with full data for our primary outcome provides mote than $85 \%$ poner to detect a significant association should one exist at an alpha kesel of 0.05 .

To determine if men and women could be combined into one analysis, sex-by-sedentary behaviour interactions were assessed for all dependent variables. Significant interactions were detected for BMI and HDI - holesterol, and thus anakyes insolving these variables are presented in men and women separately. There were no significant sex interactions for any obler variables of interes, therefore all other analyses present men and women combined in order to maximize statistical power. Normality of discribution was assessed using the Shapiro-Wilk test and visual inspection. Modkrate- and vigorous-intensicy physical activity and BMI were both transformed using a $\log$ function. Regression analyses were

Table 6. Associations ( $95 \%$ confidence interval) of baseline sedentary behaviour with 6-year change in markers of cardiometabolic risk.

\begin{tabular}{|c|c|c|c|c|c|c|c|c|c|}
\hline Model & MDL-C IM) & HDLCC (F) & LDLC & TG & FG & $\mathbf{F}$ & HOMA-IR & $\begin{array}{l}\text { Glucose } \\
\text { AuC }\end{array}$ & $\begin{array}{l}\text { Insulin } \\
\text { Auc }\end{array}$ \\
\hline 1 & $\begin{array}{l}-0.01 \\
(-0.01,0.01)\end{array}$ & $\begin{array}{l}0.01 \\
0.01,0.01 \%\end{array}$ & $\begin{array}{l}-0.01 \\
(-0.01,0.01)\end{array}$ & $\begin{array}{l}-0.01 \\
(-0.03,0.02)\end{array}$ & $\begin{array}{l}0.01 \\
1-0.01,0.01)\end{array}$ & $\begin{array}{l}0.51 \\
(-0.05,1.06)\end{array}$ & $\begin{array}{l}0.02 \\
\langle-0.01,0.05|\end{array}$ & $\begin{array}{l}-0.58 \\
(-4.07,2.92)\end{array}$ & $\begin{array}{l}-8.53 \\
(-579.88,502.82)\end{array}$ \\
\hline 2 & $\begin{array}{l}-0.01 \\
(-0.01,0.01)\end{array}$ & $\left.{ }_{\langle-0.01}^{0.01}, 0.01\right\}$ & $\begin{array}{l}-0.01 \\
{[-\infty, 1,-0.01 \%}\end{array}$ & $\begin{array}{l}-0.01 \\
(-0.03,0.02)\end{array}$ & $\begin{array}{l}0.01 \\
\mid-0.01,0.01\}\end{array}$ & $\begin{array}{l}0.67 \\
0.10,1.25)^{*}\end{array}$ & $\begin{array}{l}0.03 \\
0.01,0.05 \%\end{array}$ & $\begin{array}{l}0.06 \\
{[-3.45,3.57)}\end{array}$ & $\begin{array}{l}65.56 \\
(-524.90,656.02\end{array}$ \\
\hline 3 & $\begin{array}{l}-0.01 \\
(-0.01,001)\end{array}$ & $\begin{array}{l}0.01 \\
\langle-0.01,0.02\}\end{array}$ & $\begin{array}{l}-0.01 \\
(-0.03,0.01)\end{array}$ & $\begin{array}{l}-0.01 \\
(-0.02,0.02)\end{array}$ & $\begin{array}{l}-0,01 \\
1-0.03,0.017\end{array}$ & $\begin{array}{l}0.18 \\
(-0.99,1.35)\end{array}$ & $\begin{array}{l}0.01 \\
\langle-0.07,0.07\rangle\end{array}$ & $\begin{array}{l}-2.63 \\
{[-1251,7.251}\end{array}$ & $\begin{array}{l}-1252.36 \\
(-2527.59,22.86\end{array}$ \\
\hline
\end{tabular}

Model 1: unadjustod.

Vodel 2 adfusted for age and sex

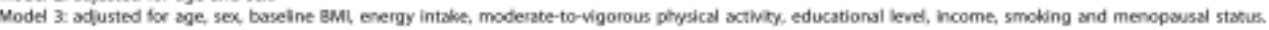

Tocas.

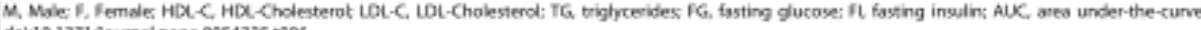

dol:10.1371/joumalpone. 0054225 .0006 
A

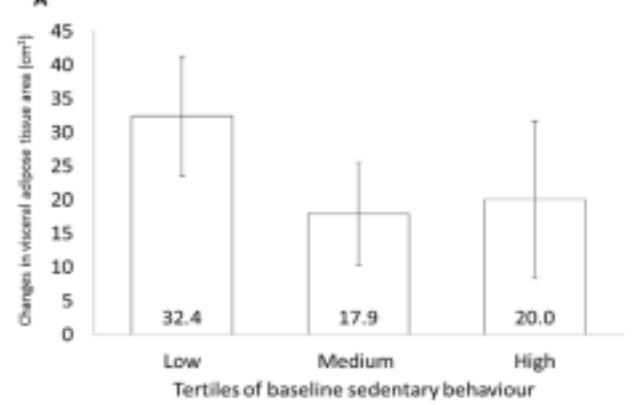

B

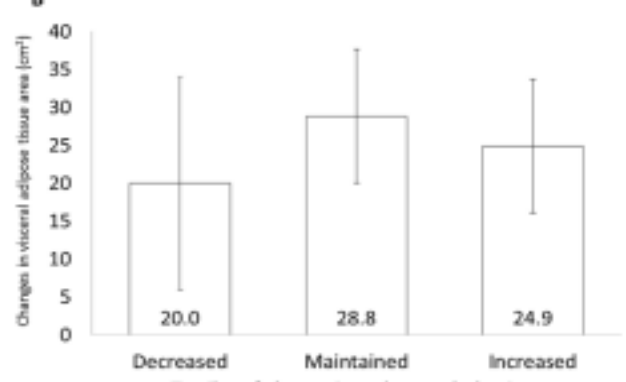

Tertiles of change in sedentary behaviour

Figure 1. Changes in visceral adipose tissue across tertiles of sedentary behaviour. Changes in visceral adipose tissue crosssectional area across tertiles of baseline sedentary behaviour (Figure 1A) or change in sedentary behaviour (Figure 18) were compared by analysis of cowariance with adjustment for age, sex, baseline BMI, energy intake, moderate-to vigorous physical activity, educational level, income, smoking and menopsusal status. Data are presented as mean \pm standard error. There were no significant dfferences across tertiles of sedentary behaviour in either anabysis.

dok $10.1371 /$ joumal.pone.005 $4225 \mathrm{~g} 001$

performed to determine the univariate and multivariate associatons between sedentary hehaviour and 6-year changes in markers of cartiometabolic risk. Multivariate models were adjusted for age, sex, baseline BMI, energy intake, moderate- and vigorous-intensity physical activity, education level, income, smoking and menopausal status. Participants were also divided into sex-specific tertiles of bascline sedentary behaviour and change in sedentary behaviour from baseline to follow-up, and an ANCOVA was then used to compare the change in markers of adiposity acroes these tertiles, adfusting for the same covariates as in the above regression analyses. It should be noted that participants with identical values were grouped into the same tertile, which resulted is unequal numbers of participants in each tertile. A Bonferroni correction was used to adjust for multiple comparisons in post hoc tests following the ANCOVA.

Data are given as mean and sandard deviation unless ochervise noted. Statistical significance was set at a $p$ value of $<0.05$. All statistical analyses were performed using SAS version 9.2 [SAS Institute, Cary, NC:

PLOS ONE I www plosone.org

\section{Results}

Baseline Sedentary Behaviour and Markers of Cardiometabolic Risk

Bascline characteristics of male and female participants are presented in Tables 1 and 2. At baseline, men and women averaged 8.3 and 7.5 bours of daily sedentary behaviour, respectisely. Sedkntary behaviour was not associated with any marker of adiposity or cardiometaholic risk in unadjusted croessectional analyses at baseline (Tables 3 and 4). These results were not changed following adjustment for age and sex. Following additional adjustment for energy intake, moderate-to-vigorous physical activity, educational level, income, smoking and menopausal status, each additional 15-minutes of baseline sedentary behaviour was croes-sectionally associated with $0.03 \mathrm{~kg} / \mathrm{m}^{2}$ lower BMI $(95 \% \mathrm{CI}=-0.05,-0.01)$ in women, but nos men. However, there were no other significant associations between sedentary behaviour and any other marker of adiposity or cardiometabolic risk in the fully adjusted model at baseline.

Tables 5 and 6 present associations of bascline sedentary behaviour with changes in measures of adiposity and markers of cardiometabolic risk, respectively, In unadjusted analyses each 15minute increase in baseline sedentary hehaviour was associated with a $0.01 \mathrm{mmol} / \mathrm{L}$ increase in HDI cholesterol $195 \% \mathrm{CI}=0.01$, $0.01)$ and $\mathrm{a}=0.04 \mathrm{~kg} / \mathrm{m}^{2}$ reduction in BMI $95 \% \mathrm{Cl}=-0.00$, -0.01 ) in women, bat not men. Following adjustment for age and sex, the association with BMII in women remained unchained, while each 15-minute increase in baseline sedentary behaviour was also associated with a $0.01 \mathrm{mmol} / \mathrm{L}$ reduction in LDL-cholesterol (95\% CI $=-0.01,-0.01)$, a $0.67 \mathrm{pmol} / \mathrm{L}$ increase in fasting insulin $\{95 \% \mathrm{CI}=0.10,1.25\rangle$, and a 0.03 unit increase in HOMAIR $(95 \% \mathrm{CI}=0.01,0.05)$ in men and women combined. However, after further adjustment for baseline BMI, energy intake, moderate-to-vigorous physical activicy, educational kevel, income, smoking and menopausal status, haseline sedentary behaviour was not assoxiated with changes in any marker of adiposity or cardiometabolic risk.

Figure $1 \mathrm{~A}$ presents the average accumulation of visceral adipose tissue across the three tertiles of baseline sedentary behaviour. The mean (standard deviation) reported sedentary time in the thee tertiles were $305(66), 471 \quad(53)$ and 642 [B9] minutes in the kow, medium, and high tertiles, respectively. There were no differences in the accumulation of any abdominal fat compartment across the three tertiles of sedentary bechaviour. Adjusting for conariates did not materially change the results.

\section{Longitudinal Changes in Sedentary Behaviour and} Markers of Cardiometabolic Risk

In the filly adjusted model, each 15-minute increase in sedentary behaviour was positively associated with a $0.13 \mathrm{~cm}$ increase in waist circumference $(95 \% \mathrm{CI}=0.02,0.25)$. However, there were no significant associations between the change in sedentary behaviour and the change in visceral adiposity or any other marker of cardiometabolic risk (data not sbown).

Participants were also classified into terriles based on their longitudinal changes in sedentary behaviour from baseline to follow up. One third of participants reduced their sedentary time by a mean (standard deviation) of $195\langle 108\rangle$ minutes over the 6 year follow-up. Another third maintained roughly the same amount of sedentary behaviour throughout the study, reducing their sedentary time by an average of just $13[39\rangle$ minutes. The final third of participants increased their sedentary time by an aserage of 165 (97) minutes. However, there were bo differences in 
the accumulation of any abdominal fat compartment among these three tertiles (Figure IB).

\section{Discussion}

Our results suggest that sedentary behaviour is not associated with 6-year changes in visceral adiposity in adult men and women. To our knowledge, this is the first longitudinal study to examine the relationship between sedentary behaviour and the accumula bon of viscral adipose tissue measured by CT. These findings are consistent with a recent study that found no cross-sectional association between objectively measured sedentary behaviour and visceral adiposity in a group of 126 abdominally obese men and women [12].

It is worth noting that both our study, and the previous study by MeGuire and Ross [12], examined the assoxiation of visceral fat with a mxasure of total scdentany time, It is unclear whether similar results would have been observed for specific modalities of sedentary behaviour (e.g, sereen-based v8, non-sereen sedentar behavioursi. For example, prospective studies in both the US and Australia have found associations between TV viewing and increased waist circumference $[7,8]$, Given that TV viewing has been linked with increased energy intake [3,28,29] this modality of sedentary behaviour may be more cloecly associated with changes in adiposity and metabolic rist than global mesasures of total vedentary time [30-32]. For example, a study of 9,000 American adultes found that those who watched more than 2 hours per day of television also consumed higher amounts of energy-dense snack foods and soft drinks, as well as consuming more calories during snacks and the evening meal [32]. Further, a recent intervention study by Harris et al. [33] reports that exposure to food adhertisements resulted in roughly a 305 increase in food intake among adult participants. Other specific forms of sedentary behaviour such as seated mental work have also been shown to result in increased food intake, as compared to simply resting in the seated position [34]. Taken together, these findings suggest that specific modalities of sedentary behaviour are likely to impact food intake (and therefore adiposity) in different ways, and highlight the importance of assessing the impact of both global sedentary bebaviour and of these specific modalities [35].

With the exception of waist circumference, the present stody also failed to detect a prospective association between sedentary behaviour and several important markers of cardiometabolic ris including plasma lipids, HOMA-IR, and glucose tolerance. These findings are consistent with some, but not all, previous prospective atudies in this area. For example, Ekelund and colleagues found no association between bascline sedentary behaviour and HOMA-IR at l-year follow-up in a group of 192 men and women [36]. In contrast, Helmethorst and colleagues reported a significant association between baseline sedentary behaviour and fasting insulin at 5-year follow-up in a cohort of 376 adults, independent of physical activity levels [37]. Of note, both of these studies used objective measures of total sedentary time at baseline. Wijndack and colleagues have also reported prospective associations betweet

\section{References}

1. Tremeday MtS Cadey RC, Sunders T]. Healy GN, Owen N [2010

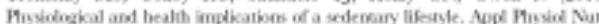
Mreab 50: 725-740.

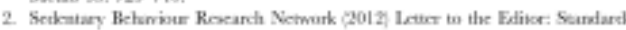

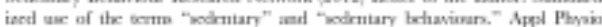

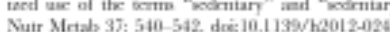

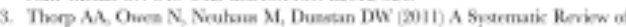
Longmadnal Studies, 1960-2011. An J Prev Med 41; 20j-215. changes in television viewing and clustered cardiometabolic risk in women, but not men [8].

As with adiposity, it is likely that the relationship between sedentary bebaviour and markers of cardiometabolic risk may also vary depending on the modality of sedentary bebaviour. For example, we have previously reported that seated video-game use, but not seher forms of sedentary behaviour, arr asociated with imcreased metabolic risk in overweight and obese adolescents [38]. Given these and ocher findings, it is difficult to come to global conclusions regarding sedentary behaviour and the developmen of subsequent cardiometabolic risk. However, given the consistent associations between sedentary behaviour and the risk of mortality reported in other studics, public bealth messages promoting reductions in sedentary behaviour remain important [3].

The present study contains strengths and weaknesses that warrant mention. Limitations include the measurement of sedentary behaviour by self-report, and a lack of information related to specific modalities of sedentary behaviour. The observed results may have differed if an objective measure of sedentary behaviour had becen emploned, or if seckentary behaviour had becen broken into specific modalities such as screen-time and non-screen sedentary behaviours. The relatively small sample size and homogencous sample of the current study atso limits our statiscical power, and the generalizability of these findings. It should also be noted that this was a retrospective analysis, as the Quebec Family Study was originally designed to assess the genetic contributions to obesity. Strengths of this study include its longitudinal design and the use of computed tomography to assess visceral and subcutaneous abdominal adiposiny [39]. This study also included objective measures of several important markers of cardiomptabolse risk, including lipidk, insulin resistance, and glucose tolerance in both men and women srudied in their natural environment.

In summary, our results suggest that peither baseline sedcntary behaviour nor changes in sedentary behaviour are associated with longitudinal changes in visceral adiposity in adult men and women. With the execption of waist circumference, sedentary behaviour does not appear to be associated with longitudinal changes in any marker of cardiometabolic risk in this population. These findings suggest that the developenent of cardioenetabolis risk may be due primarily to factors otber than self-reported sedentary behaviour.

\section{Acknowledgments}

The autben wish to thank the stach participants and the staff of the Pheviral Activity Scirnors I ahoratory at Iaval University for contributing to this study. The authers specially thank G. Fournier, Dr G. Therisult, 1. Alard, M. Chagnoe and C. Leblane for their contributioes to the recruimsent and data collection of the Quebee Family Study.

\section{Author Contributions}

Designed the satistical analyes: TJS JPC MST. Revied manuerim: JPC MST CB AT JPD TJS, Coscrived and designed the esperiments CB.JPD AT. Performed the experiments: CB JPD AT. Anahred the data: TJS Wrote the paper: TJS.

4. van de Poez HP, Chey T, Konda RJ, Banks E, Bowman A [2012, Siming Tim

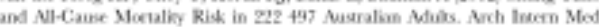

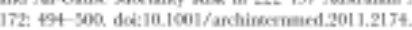

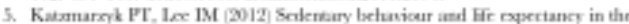
US

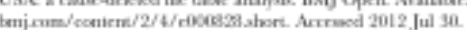

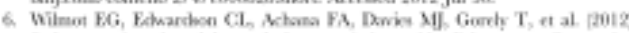
Sodenary time in addits and the asociative whe dibetes, candwasuber

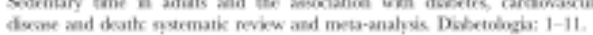




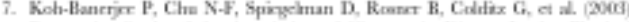

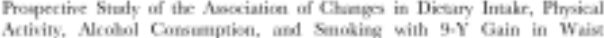
Groumfirence Amoes 16587 US Men. Am J Clin Natr 78: J19-727.

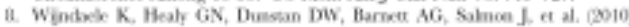

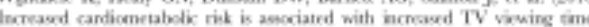
Mnd Sri Spros Exerr 42. 1511.

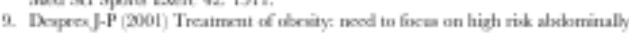

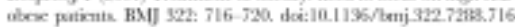

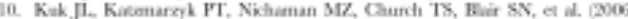

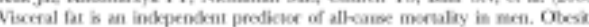

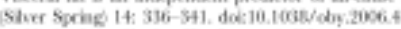

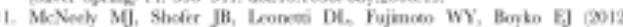

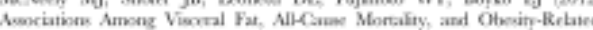

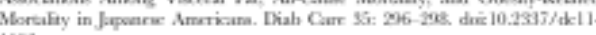
1193.

12. MeGuire KA, Ress R [2011) Incidemal Plmical Activity and Sodenary

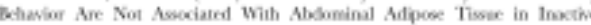

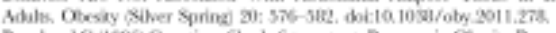

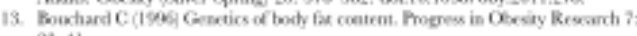
33- 41 .

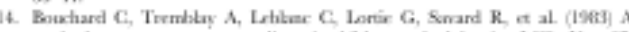

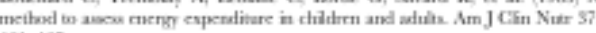
651-467.

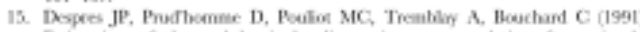
Estimation of dep abdominal adipose-thaje accumulation from simph

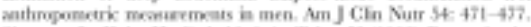

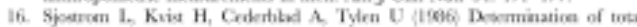

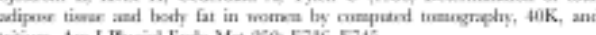

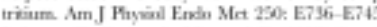

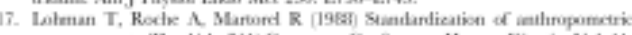

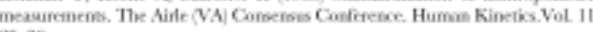
39-30.

11. Ketinke AR, Wilmoe JH (1974) Exaluation and regulation of body beidd and

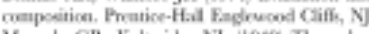

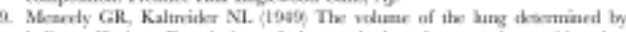

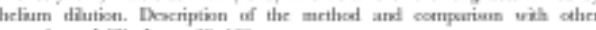
prosoduro. J Clin Imest 28 129.

20. Siri WE (1956) The gros composite of the body. Mtences in biobgical and medical phrosis $t 238$

1. Peruse L, Despees JP. Tremblay A Latian C, Tahos J, ef al (1909) Genetk

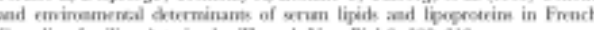

22. Pirume L, Eice T, Dequro JP, Bryzron J. Prosincx XLA, et al. (1997) Familia

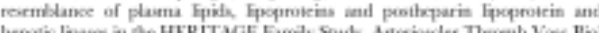

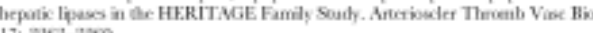

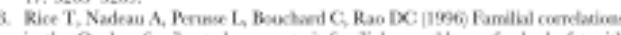

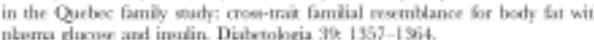

24. Oppert JM, Nadrau A, Trembitry A, Dospris JP, Therriult G, at ad. (1995)

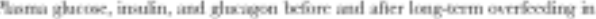

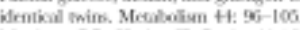

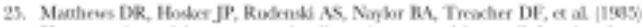

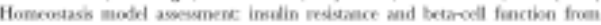

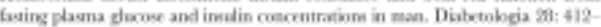
419 .

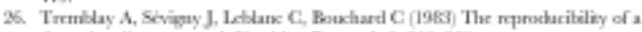
there-day dictary roord. Nutrition Rrourch sa 819 ase

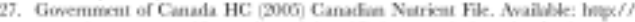

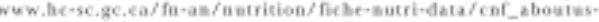

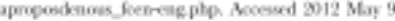

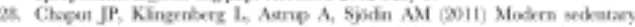
artivities promote owrenasamprion of food in our current desong=ir envionment. Otasiy Kretiem 12: $12-20$

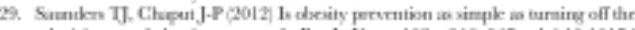
iclesision and liaving a nap?' Bt J Nuir 108: 946-947. doé10.1017J soñ114512008itt

90. Carsen V, Jansen 1 (2011) Vedianc, panens, and pros of sedenary betasioe

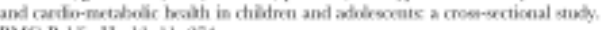
BMC Puthir Healith $11=274$

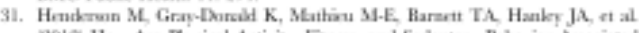

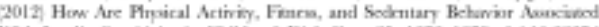

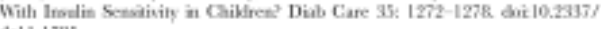
de11-178s.

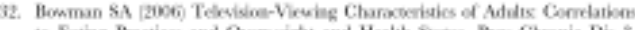

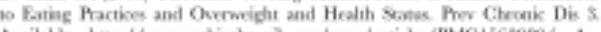

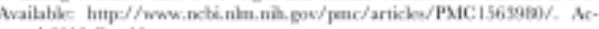
coeved $2012 \mathrm{Oct}$ is.

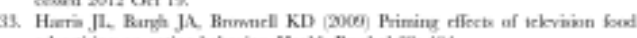
athertiaing an eating bethavier. Healh Probol 28: 404

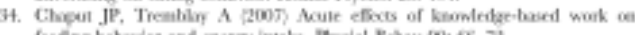

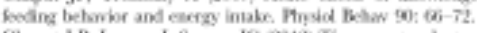

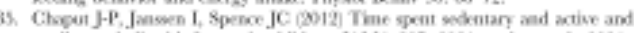

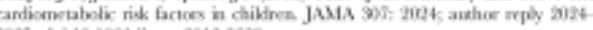
ins. dui 10.1001/jama.2012.5i24.

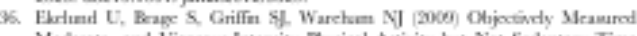

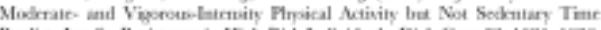

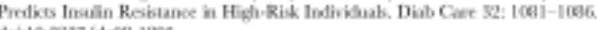
bot. $10.2357 / \mathrm{dcc} \theta-1890$

37. Heimesters HIF, Wiodveb K, Broge 8, Warcham N]. Eladued U poos Objectively Sleasured Sedentary Time May Predict Insula Kesintance

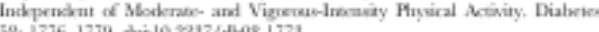

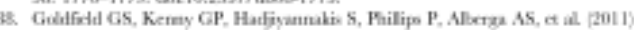

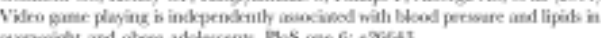

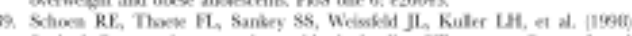

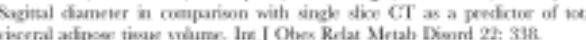




\title{
Clustering of children's activity behaviour: the use of self-report versus direct measures
}

Travis J Saunders", Stephanie A Prince and Mark S Tremblay

\begin{abstract}
While we concur with the objectives of the recent intemational Joumal of Behovioural Mutrition and Physical Activity paper published by Jaoo and colleagues titled "Physical activity and sedentary behaviour typologies of 10-11 year olds", we feel that the results as currently presented do not support their conclusions. Though the authors created groups of children with dramatically different patterns of self-reported physical activity and sedentary behaviour, an inspection of the objectively measured accelerometry data shows little difference between the groups. Further, in at least one instance the difference between groups was of the opposite direction when using objective measures as opposed to the self-report measures used in the published analysis. Thus, we caution the authors from making conclusions based on their self-report data, and propose that they re-analyze their data using their objectively measured data instead.
\end{abstract}

To the Editor,

We read with great interest your recently published study by Jago and colleagues [1] titled "Physical activity and sedentary behaviour typologies of $10-11$ year olds". The authors argue convincingly that interventions which aim to promote increased physical activity and/or reduced sedentary behaviour should focus on the specific needs and characteristics of their target populations. As such, we concur that their objective to identify clusters of children with similar patterns of physical activity and sedentary behaviour would provide key information for the design of targeted interventions. Unfortunately, we believe that the data presented in the paper suggests that the clusters created by the authors do not represent groups of children with distinct activity patterns, and that the conclusions of the paper are therefore unsupported.

In their paper, Jago et al. [1] assessed physical activity and sedentary behaviour using both self-report questionnaires and accelerometry. However, when creating clusters of children with similar behaviour, the authors relied on only the self-reported data. While this resulted in clusters of children with very distinct quantities of self-reported physical activity and sedentariness, the

- Comerpondence

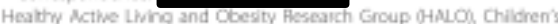

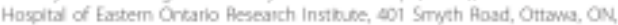

K1H \&L. Cangda

() BioMed Central

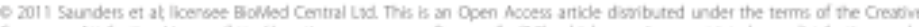

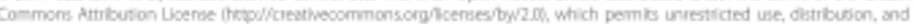
rearoduction in ary medium, prowided the origind work is proserty cted. 
Activity/Medium Sedentary" group on both weekdays and on weekends. Further, it is questionable whether it would even be possible for children to accumulate the daily volume of screen time (13.86 hours) and physical activity ( 5.89 hours) reported by children in the "High Activity/High Sedentary" cluster. If true, this would leave the children less than 5 hours per day for both school-work and sleep, suggesting that these values are not just unlikely but impossible.

The large discrepancies between objective and selfreport activity patterns observed in the present study have also been reported by others. For example, a recent systematic review by Adamo and colleagues [2] reports that, in comparison to accelerometry, self-report mea sures overestimate physical activity by an average of $114 \%$ in boys and $584 \%$ in girls. Recent findings also suggest that self-reported screen time is only weakly correlated with objectively measured sedentary behaviour in adults [3]. It has also been noted that few of the studies which purport to assess sedentary behaviour have actually measured it [4]. Given the discrepancies between self-report and direct measures of activity in the literature, and the availability of directly measured data in the present situation, we caution the authors from making conclusions based on their self-report classifications. Further, we would be interested to know how the behaviour clusters created in the present study might differ if they were based on the accelerometry data, and whether this might also result in more pronounced differences between the clusters in terms of body mass index or the Index of Multiple Deprivation score.

We welcome comments from the authors of the current study in order to provide further clarification of the methods employed and conclusions made.

Respectfully,

Travis I. Saunders, Stephanie A. Prince and Mark S. Tremblay.

Acknowiedgements and Funding

TSS is supported by a Doctord Fesearch Aumand from the Conadion hetitutes of Health Research and a Universicy of Onawa Ercelence Scholarthip. SAP is supported by an Orcario Graduate Scholarshipa a Universioy of Otrawa Excellence schotastip and by Doctaral Roseanch Awards from the Social sciences and Humanities Hesearch Councl of Canada and fom the University of Ottawa. The funding agencies did not plas a nole in the decision to sutarit this manuscript for publication.

Authors' comriautions

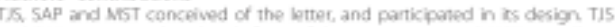
and SAP participated in the drafting of the maruscript, and MST cortically

revised it. All authors read and apporand the final manuscripe.

Competing interest

The authors declase the ther hare no competing irveregs.

Feceined: 27 lanuary 2011 Accepted: 25 May 2011 Published 25 May 2011
References

Jago R. For KR Page AS, Brockman R. Thompson l. Phrsical activity and

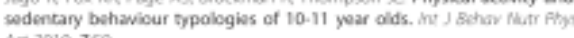
Act 2010, 759 .

2. Adamo KB, Prince SA, Tricco AC, Connor Gorber S, Tremblyy MSE A comparison of direct versus self-report messures for assessing physical activity in the pediatric population a systemotic review. Int $f$ Ped Obesiy 2006, 4(1)2-27

3. Cark BK Healy GN, Winider EA, Gardiner PA Sugjama T, Dunstan DW, Matrhews CE, Omen N Relationship of velevesion time with accelerometer-derived sedentary time: NHANES. Med Sci Sports Evenc $2011,43 / 5)$ : 222 -

4. Thembloy MS, Colley AC, Sounders Td, Hesthy GN, Omen N: Physiological and health implications of a sedentary lifestyle Apol Prosiol Notr Mesot $201035 \pi 5-740$

doi:10.1 186/1479-5so6-5-48

Che this article as: Saunders ef oc Clustering of chaldren's activety

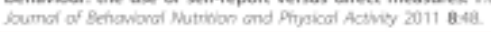

Submit your next manuscript to BioMed Central and take full advantage of:

- Converient online submission

- Thorough peer review

- No space constraints or color tigure charges

- Immediate publication on accoptance

- Inclusion in Pubned, CAS, Scopus and Google Scholar

- Research which is freely available for redistribution

Submit your manuseript at

( ) Biomed Central 


\title{
Review Article
}

\section{Potential Contributors to the Canadian Pediatric Obesity Epidemic}

\author{
Travis Saunders ${ }^{1,2}$ \\ 'Healthy Active Living and Obesity Research Group, Children's Hospital of Eastern Ontario Research Institute, Ottawa, \\ ON, Canada K1H $8 L 1$ \\ School of Human Kinetics, University of Ottawa, Ottawa, ON, Canada K1N 6N5 \\ Correspondence should be addressed to Travis Saunder \\ Received 12 September 2011; Accepted 24 October 2011 \\ Academic Editors: R. Hentschel, J. A. O'Neill, and D. D. Trevisanuto \\ Copyright $\otimes 2011$ Travis Saunders. This is an open access article distributed under the Creative Commons Attribution License, \\ which permits unrestricted use, distribution, and reproduction in any medium, provided the original work is properly cited. \\ As a group, Canadian children and youth are heavier than at any time in the recent past. However, to date there has been no critical \\ examination of the factors which are likely to have contributed to these deleterious trends. A review of the evidence suggests that \\ there is robust evidence supporting the role of reduced sleep, increased sedentary time, increased consumption of sugar-sweetened \\ beverages, and secular increases in adult obesity as contributing factors to the current epidemic of childhood obesity. There is \\ moderate evidence that these trends are related to changes in either total energy intake or physical activity, while there is very little \\ evidence supporting the role of maternal age, breastfeeding, exposure to endocrine disrupters, or inadequate calcium intake. These \\ findings suggest that targeting sleep, sedentary time, and sugar-sweetened beverage intake in Canadian children and youth may \\ help to prevent future weight gain at the population level.
}

\section{Introduction}

Available evidence suggests that Canada is in the midst of an epidemic of childhood obesity [1-4]. Between 1981 and 2007-2009, the average body mass index (BMI) of 12-yearold Canadians increased from 18.1 to $19.2 \mathrm{~kg} / \mathrm{m}^{2}$ in boys, and from 18.4 to $19.5 \mathrm{~kg} / \mathrm{m}^{2}$ in girls [4]. During this same time period the prevalence of overweight/obesity among Canadians aged 15-19 increased dramatically from $14 \%$ to $31 \%$ in boys, and from $14 \%$ to $25 \%$ in girls [4]. In fact, among Canadians aged $15-19$, fully $14 \%$ of boys and $10 \%$ of girls are now considered obese [4]. Equally worrying, as BMI has increased during the past 30 years, so too has the prevalence of abdominal obesity $[1,4]$. Since 1981 , the average waist circumference (WC) among Canadian youths aged 12-19 has increased by more than 5 centimetres, such that approximately one-fifth of Canadians in this age group now have a WC that places them at some form of increased health risk [1].

These recent increases in both the BMI and WC of Canadian youth are a tremendous public health concern, as pediatric obesity is associated with both metabolic dysfunction in childhood [5-8], as well as an increased risk of mortality well into adulthood $[9,10]$. Thus, the objective of the present paper is to give a comprehensive examination of the possible causes of our current epidemic of childhood obesity. This will be done through a discussion of the strength of the evidence base and biological plausibility for each putative factor, before finally comparing their relative contributions to these deleterious trends. This review will focus on Canadian data whenever possible, Before examining the putative causes of the epidemic, however, it is important to briefly review the factors affecting energy balance and their role in body weight.

\section{Energy Balance}

As noted by Jéquier and Tappy, the first law of thermodynamics-which states that energy can neither be created nor destroyed-applies to humans [11]. With respect to body weight, this means that changes in stored energy (e.g. 
adiposity) are equal to energy intake (EI) minus energy expenditure (EE) [11]. Energy expenditure can be broken down into three separate components [11]:

(i) basal metabolic rate (BMR),

(ii) diet-induced thermogenesis, and

(iii) energy used for exercise and physical activity (PA).

Energy intake, on the other hand, is simply the sum of the energy consumed by an individual, minus approximately $5-10 \%$ that is excreted in urine and feces [11]. When EI exceeds EE, the result is an increase in energy stores, and therefore weight gain. Thus, any putative cause of the childhood obesity epidemic must influence either EI, EE, or both. With that in mind, let us now evaluate the role of both conventional and unconventional factors in the etiology of childhood obesity.

\section{Reduced Physical Activity}

Given its role in the energy balance equation it is quite obvious that, all else being equal, a reduction in the number of calories burned through PA will directly lower EE and result in positive energy balance. Regular bouts of PA are also known to result in substantial elevations in BMR in both lean and obese individuals $[12,13]$, suggesting that reductions in $\mathrm{PA}$ may further reduce $\mathrm{EE}$ by deleterious changes to BMR. Similarly, it has also been suggested that regular PA results in more accurate coupling of EI and EE [13-17]. Taken together, these findings suggest that reductions in PA may negatively impact both sides of the energy balance equation by directly reducing $\mathrm{EE}$ and by inhibiting the proper regulation of EI. Not surprisingly, available observational evidence also suggests that PA plays a role in the prevention of excess weight gain in children and youth.

Numerous cross-sectional studies report that overweight and obese children are less active than their lean peers, while the majority of longitudinal studies report small, inverse associations between high levels of PA and the accumulation of excess body weight [18-20]. For example, Berkey and colleagues report that every hour of self-reported daily PA in girls aged $9-14$ is associated with a $-0.0284 \mathrm{~kg} / \mathrm{m}^{2}$ smaller increase in BMI over a one-year period (the relationship was of borderline significance in boys) [21]. Similarly, a recent systematic review by Connelly and colleagues reports that compulsory PA is the single most defining factor of controlled trials that successfully prevent the development of childhood overweight or obesity [22]. It should be noted that current findings are based mainly on self-reported levels of PA, which are known to be substantially less accurate than objective measures such as pedometry and accelerometry $[23,24]$. However, despite these methodological limitations, the balance of evidence suggests that low levels of PA are likely to predispose to future weight gain.

While the above evidence suggests that low levels of PA are likely to result in increased risk of future weight gain, at present it is unclear whether current levels of PA in Canadian youth are lower than those of previous generations, which would be necessary in order for PA to play a causal role in the current obesity epidemic [25]. Self-reported leisuretime PA among Canadian adolescents actually increased during the 1980 's and remained stable throughout the 1990 's [26], suggesting that current PA levels may be higher than they were before the obesity epidemic. However, this data conflicts with other lines of evidence, which suggest that total PA levels among Canadian children may be lower than they were in the past. For example, it has been reported that the proportion of trips to school that involves active transportation decreased by roughly $20 \%$ between 1986 and 2006 among Canadian children in the Toronto region [27]. Similarly, children who live in Canadian Old Order Amish and Mennonite communities, where lifestyles are similar to those in contemporary Canadian society $60-100$ years ago [23], accumulate roughly $50 \%$ more steps per day than their contemporary Canadian peers $[28]$, as well as $30-$ $50 \%$ more moderate-to-vigorous PA [29]. In the absence of more complete and objective data on the PA of past generations of Canadian youth and given that less than $10 \%$ of Canadian youth are currently meeting PA guidelines [3032 , it appears relatively safe to conclude that total PA-related EE of Canadian youth is at or near historic lows.

Thus, given the multiple biological mechanisms linking reduced PA with increased adiposity, consistent but relatively small longitudinal associations between PA and weight gain, and evidence suggesting that Canadian children are likely less active than in previous generations, there is currently moderate evidence that insufficient PA plays a causal role in the current epidemic of childhood obesity.

\section{Increased Sedentary Behaviour}

Sedentary behaviour is defined as "a distinct class of behaviours (e.g., sitting, watching TV, driving) characterized by little physical movement and low energy expenditure (s1.5 METs)" [33]. At present, it is unclear whether the prevalence of sedentary behaviours in Canada and other industrialized nations has increased in recent decades. For example, a review by Marshall and colleagues reports that the screen time (time spent watching television, playing videogames, or using computers) of children in modern nations has not increased since the 1950's [34]. In contrast, Nelson and colleagues report that weekly computer usage increased by roughly 4 hours/week between 1999 and 2004 in American youth [35]. Similarly, a recent report suggests that Canadian children average more than 6 hours of screen time on weekdays, and that even preschoolers watch an average of almost 2 hours of television per day [36] — amounts that seem highly unlikely 40 years ago. Similar trends are seen in sedentary modes of transportation such as driving, which have also increased dramatically in recent decades [27]. Finally, accelerometry data from the nationally representative Canadian Health Measures Survey suggests that Canadian youth spend an average of 8.6 hours per day (more than $60 \%$ of their waking hours) engaging in sedentary behaviour [30]. Taken together, these reports suggest that Canadian children are likely more sedentary than previous generations.

In addition to reports of increasing levels of sedentary behaviour in Canadian youth, there is also an accumulating body of evidence which suggests that high levels of sedentary behaviour may predispose to weight gain, especially in 
young children, while reductions in sedentary behaviour may promote weight loss or weight maintenance $[18,37]$. For example, Burke et al. [38] report that every hour of television watching at age 6 was associated with a $50 \%$ increased risk of overweight or obesity at age 8 in a sample of Australian children, independent of other risk factors for weight gain. Similarly, a randomized controlled trial which reduced screen time resulted in significant reductions in both weight gain and the accumulation of abdominal fat in elementary school children [39]. However, few studies have found relationships between sedentary behaviour and weight gain in older children, suggesting that sedentary behaviour may only be a risk factor for obesity in young children $[18,37]$.

The potential mechanisms which are thought to link sedentary behaviour and adiposity involve deleterious changes to both EE and EI. Most obviously, sedentary activities are defined by having low EE [33] and may also displace PA, although there is currently little evidence that such displacement takes place $[18,40-42]$. With respect to $\mathrm{EI}$, excess sedentary behaviour may also result in "uncoupling" between EE and EI [14]. Further, it has been suggested that television viewing may exert a particularly negative impact on pediatric EI. For example, Wiecha and colleagues report that every one-hour increase in daily television viewing among school children is associated with an extra consumption of 167 calories [43]. In addition, it has also been suggested that exposure to television food advertisements increases children's food intake at subsequent meals $[44,45]$. Thus, through its impact on both EE and EI, it is very plausible that sedentary behaviour has a deleterious impact on energy balance, and therefore body weight.

Given the strong and consistent relationship between sedentary behaviours and weight gain in early childhood, temporal trends which suggest that sedentary behaviours have increased in recent decades, and numerous plausible biological mechanisms, there is currently strong evidence that increases in sedentary behaviour play an important role in the epidemic of childhood obesity.

\section{Increased Total Energy Intake}

It is well established that intentional overfeeding results in significant weight gain [46-48]. For example, Levine and colleagues report that overfeeding volunteers by $1000 \mathrm{kcal} / \mathrm{day}$ results in an average weight gain of $5 \mathrm{~kg}$ in just 8 weeks [47]. However, counterintuitively, recent reviews have noted that total EI has not been a consistent predictor of weight gain in prospective studies of children $[19,49]$. It is worth noting however that this may be due to the limitations of self- or parent-reported caloric intake, as both adults and children are known to have great difficulty in accurately reporting EI [50-52]. For example, Huang and colleagues report that fully $55 \%$ of children aged 3-19 who participated in the Continuing Surveys of Food Intakes by Individuals study reported physiologically implausible values for energy intake, and that excluding these individuals resulted in dramatic improvements in both the strength and the consistency of the relationship between EI and body weight [52].
As with PA, there is currently little information regarding historical trends in the EI of Canadian children, and data from the United States are equivocal as some [53-56], but not all $[57,58]$, studies report increased EI during the past half century. For example, self-reported energy intake from the 1977-1978 Nationwide Food Consumption Survey and the 1999-2004 National Health and Nutrition Examination Survey suggest that the average daily EI of American children aged $1-10$ in $1999-2004$ was $15 \%$ higher than in 1977-1978, with similar increases observed in adolescents $[54,55]$. In contrast to these findings, however, Troiano and colleagues report that between 1970 and 1994, EI in American youth was relatively stable [57]. Thus, given the absence of Canadian data and the ambiguity of available data from our closest neighbour, there is currently insufficient evidence to conclude that EI has increased in Canadian youth during recent decades.

While prospective studies and historical trends may lend only weak support to the putative role played by EI in the Canadian childhood obesity epidemic, it remains extremely plausible biologically. As mentioned earlier, several trials have shown that intentional overfeeding results in dramatic weight gain in adults [46-48], and there is little reason to expect this relationship to be different in children. While it should be noted that there is evidence that overconsumption results in compensatory increases in $\mathrm{EE}$ in some individuals, [47], this would likely be insufficient to prevent an increase in obesity rates at the population level. Further, other consistent predictors of weight gain which lend themselves to more accurate self-reporting than total EI (e.g., television watching and sugar-sweetened beverages, which will be discussed below) are thought to exert their influence through their impact on EI. Thus, despite the weak evidence presented from observational studies, the strong biological plausibility and impressive results from studies of chronic overfeeding suggest that there is currently moderate evidence that increased EI has contributed to the childhood obesity epidemic.

\section{Increased Sugar-Sweetened Beverage Intake}

While trends in total EI over the past 40 years are unclear, there is little ambiguity for trends in sugar-sweetened beverage (SSB) intake, which has increased dramatically in recent decades [58-60]. For example, the average self-reported softdrink intake in American youth increased from roughly $150 \mathrm{~mL} /$ day in 1977 to more than $350 \mathrm{~mL} /$ day in 1998 [59], and recent studies suggest that total SSB intake has continued to increase into the 21st century [60]. Interestingly, while this may be partially due to increased fast food consumption, available evidence suggests that SSB intake has also increased in the home environment in recent decades [56].

Several recent systematic reviews have also concluded that there is consistent evidence that excess consumption of SSBs is associated with an elevated risk of weight gain $[19,61$, 62]. For example, among longitudinal studies, Vartanian and colleagues report significant effect sizes of 0.24 and 0.09 for the relationship of SSB consumption with total EI and body 
weight, respectively [62]. Similarly, a 19 -month prospective study of 548 school children reports that every serving of sugar-sweetened beverages at baseline was associated with a $0.18 \mathrm{~kg} / \mathrm{m}^{2}$ increase in BMI at followup [63]. Finally, it has recently been estimated that removing sugar-sweetened beverages from the diet of American children and youth would reduce caloric intake by an average of 235 calories per day [64], which has the potential to dramatically reduce the risk of positive energy balance in this age group. Thus, available evidence suggests that excessive consumption of SSBs plays a strong role in the etiology of the childhood obesity epidemic

This relationship between SSB and prospective weight gain can be explained by multiple biological mechanisms. First and foremost, SSB intake is associated with increased EI, as described earlier [62]. This is likely due to the fact that SSBs are both energy dense and have little impact on satiety, both of which could lead to increases in EI [61]. Further, many SSBs are sweetened with high fructose corn syrup (HFCS) and therefore contain a fructose fraction. This fructose fraction may also contribute to weight gain through increased lipogenesis, inhibition of satiety signals, and reductions in EE, although it should be noted that the relative importance of HFCS in the etiology of obesity is stil a matter of dispute $[61,65]$.

\section{Increased Dietary Fat Intake}

Not surprisingly, as the consumption of carbohydrates has increased during the past 30 years, the relative contribution of fat to total EI has decreased, although intake remains above recommendations $[57,58]$. For example, Cavadini and colleagues report that between 1965 and 1996 fat intake decreased from $39 \%$ to $32 \%$ of EI for Americans aged $11-$ 18 [58]. However, it should be noted that this same study found that absolute fat intake actually increased by $4 \%$ during the 1990's [58], suggesting that the relative changes in fat intake may have more to do with increased consumption of carbohydrates than with reductions in fat consumption.

The evidence linking fat intake and obesity in prospective studies is surprisingly equivocal and provides little support for the role of fat intake in the development of obesity [19]. For example, Davis and colleagues report that of 15 longitudinal studies of childhood weight gain reviewed by the American Dietetic Association, just 4 supported the role of dietary fat intake, while 4 others showed mixed results, and 7 found no association [19]. However, as with total $\mathrm{EI}$, fat intake remains an extremely plausible mechanism biologically. Similar to SSBs, dietary fat is both energy dense and relatively nonsatiating per calorie ingested, lending itself to passive overconsumption [66]. Further, increased fat consumption appears to have little impact on fat oxidation or overall EE, suggesting that excess EI related to increased fat intake is very likely to result in positive energy balance and weight gain [67]. However, despite these plausible mechanistic links, available evidence provides only weak support of the role of dietary fat intake in the current childhood obesity epidemic.

\section{Reduced Calcium Intake}

Along with the changes in fat and SSB intake in recent decades, there has also been a well-documented reduction in the intake of dietary calcium $[58,68]$. For example, between 1965 and 1996, Cavadini et al. report that the milk consumption of American youth decreased by $36 \%$, while total calcium intake dropped by roughly 13\% [58]. Further, it has been suggested that high calcium intake may influence body weight through increases in fecal fat excretion, fat oxidation, and thermogenesis $[69,70]$. However, a recent meta-analysis of childhood calcium supplementation studies reports no significant association between supplementation and any measure of weight or body composition [71], which is supported by the findings of a similar review in adults [72]. Thus, while there is some evidence for plausible mechanisms linking reduced calcium with increased adiposity, the lack of evidence linking calcium intake with changes in actual measures of body composition suggests that reductions in calcium intake do not represent an important cause of the Canadian childhood obesity epidemic.

\section{Reduced Sleep}

Available evidence suggests that short-sleep duration may be another important risk factor for childhood overweight and obesity. A recent systematic review and meta-analysis by Cappuccio and colleagues reports that children who sleep less than 10 hours per night are at $89 \%$ greater risk than their peers who sleep more than 10 hours/night [73]. Using this same data, it has been estimated that 5 to $13 \%$ of all childhood obesity could be due to short-sleep duration [74]. Although the vast majority of the research to date has been cross-sectional [73], there is evidence of sleep as a predictor of weight gain in prospective studies as well. For example, Reilly and colleagues report that toddlers who slept less than 11 hours per night at age 2.5 years were $35-45 \%$ more likely to be obese at age 7 than toddlers who averaged more than 12 hours of sleep [75], with similar findings reported by Bell and Zimmerman [76].

Secular trends in sleep duration also support the putative role of sleep duration in the childhood obesity epidemic. Since the 1970's, the average sleep duration of children has decreased significantly among industrialized nations. Between 1974 and 1986, the average sleep time of 2year olds in the Zurich Longitudinal Studies decreased by 45 minutes [77], while Dollman and colleagues report a 30-minute decrease from 1985 to 2004 among South Australian teenagers [78]. Similarly, the prevalence of sleeponset difficulties has also increased dramatically in recent years [79].

Finally, a putative role for shortened sleep in the etiology of the obesity epidemic is also supported by plausible mechanisms which are thought to influence both EE and EI $[80,81]$. For example, it has been reported that sleep restriction in adults results in significant increases in hormones which promote EI including cortisol and ghrelin, along with decreases in anorectic hormones such as leptin and PYY $[80,82-84]$. Not surprisingly, short-sleep duration has also 
been shown to result in increased hunger and appetite, both of which were strongly associated with the changes in ghrelin and leptin mentioned earlier [84]. Given that leptin and ghrelin are thought to, respectively, promote and inhibit physical activity, it has been suggested that sleep debt could potentially result in reductions in $\mathrm{EE}$ as well $[81,85]$. However, recent experimental evidence in young men suggests that acute sleep restriction results in relatively little change in EE [86]. Thus, at present it appears very likely that sleep deprivation results in increased EI, while there is little direct evidence that it results in reduced EE. When these biological mechanisms are considered alongside the consistent relationship between shortened sleep and obesity in prospective studies, and secular trends in sleep duration, there is currently strong evidence that shortened sleep plays a role in the childhood obesity epidemic.

\section{Prenatal Exposure to Endocrine-Disrupting Chemicals}

Endocrine-disrupting chemicals (EDCs) are any "compound, either natural or synthetic, which alters the hormonal and homeostatic systems that enable the organism to communicate with and respond to its environment" [87], several of which (known as obesogens) may influence body weight [88]. Limited evidence suggests that EDCs may exert a negative influence on aspects of $\mathrm{EE}$. For example, it has been reported that mothers who have high levels of polychlorinated biphenyls (PCBs) in their breast milk also have low levels of plasma triiodothyronine, a thyroid hormone which is known to stimulate basal metabolism [89]. Similarly, interventions in adults which increase plasma organochlorine concentrations result in significant decreases in both triiodothyronine and resting metabolic rate [90] and may also reduce skeletal muscle oxidative capacity [91]. However, despite this limited biological evidence linking EDCs and EE, at present it is unclear whether prenatal exposure to EDCs predisposes to future weight gain [92]. For example, while some reports suggest that the concentration of PCBs in cord blood is positively associated with BMI in early childhood [92], other reports suggest no relationship [93], or even a negative relationship [94] between prenatal PCB exposure and prospective weight gain. Similar inconsistencies have also been observed for other EDCs such as DDE [92]. Thus, while being an interesting area for future research, at present there is very little evidence that EDCs play a causal role in the childhood obesity epidemic.

\section{Increased Maternal Age}

The average age of first pregnancy has increased dramatically in recent decades in both Canada [95-97] and around the world [98-100], and several plausible mechanisms have been suggested, which could link maternal age with increased risk of childhood obesity. For example, older mothers are known to give birth to smaller infants, which is itself a risk factor for the development of obesity $[96,101]$. Similarly, older women are also likely to have both higher plasma concentrations of EDCs and higher BMIs, both of which may also predispose their children to future weight gain, as discussed elsewhere in this review [102-104]. Finally, research in sheep suggests that older maternal age may result in increased fat deposition [105], which may be related to accelerated reductions of proteins responsible for thermogenesis-related energy expenditure [25], although it is not immediately clear how or if this relates to humans.

Although the mechanisms described above are all at least somewhat plausible, the relationship between maternal age and childhood obesity in observational studies is inconsistent. For example, while Patterson and colleagues report that the odds of obesity in a cohort of American girls increased by $14 \%$ for every 5-year increase in maternal age [106], a more recent study of 8234 British children found no relationship between maternal age and risk of obesity at age 7 [75]. Given this conflicting evidence, there is currently only weak evidence that maternal age plays a role in the childhood obesity epidemic, and future prospective studies are needed to clarify this relationship.

\section{Reduced Breastfeeding}

Duration of breastfeeding has been strongly and consistently linked with reduced risk of childhood overweight and obesity [107]. For example, Harder and colleagues performed a meta-analysis which examined the association between duration of breastfeeding and the risk of childhood overweight in 17 independent observational studies [107]. In comparison to children who were breastfed for less than 1 month, they report that children who were breastfed for 1-3 months had $19 \%$ reduced risk of overweight. The risk of being overweight continued to decrease as the duration of breastfeeding increased-risk was reduced by $24 \%$ among those breastfed for 4-6 months, $33 \%$ among those breastfed for 7-9 months, and by $50 \%$ for those breastfed for more than 9 months. On average, each additional month of breastfeeding reduced the risk of being overweight by $4 \%$.

Despite consistent reports of the relationship between breast feeding and reduced risk of overweight and obesity, the mechanisms underpinning this relationship remain unclear. It has been suggested that it may be due to alterations in the neuroendocrine control of appetite, although this has yet to be verified in human participants [107]. Thus, it is not possible at present to determine the precise mechanisms linking the duration of breastfeeding to body weight in childhood.

While breastfeeding appears to have a strong relationship with the risk of excess weight gain in childhood, trends in the prevalence of breastfeeding suggest that it is not a major contributor to secular increases in childhood obesity rates during the 20th century. Since the 1970's, the prevalence of breastfeeding has remained constant or increased among most western nations for which data is available $[108,109]$. For example, in the early 1970's roughly $20 \%$ of American women exclusively breastfed while in the hospital, but this increased to $45 \%$ by the year 2000 [109]. Given that obesity rates continued to increase steadily throughout this period 
despite increases in the prevalence of breastfeeding, there is currently weak evidence that breastfeeding plays a primary role in the childhood obesity epidemic.

\section{Increased Adult Obesity Rates}

Available evidence suggests that both parental obesity and gestational weight gain are risk factors for childhood obesity $[75,110,111]$. For example, Reilly and colleagues examined the relationship between parental and childhood obesity in a prospective study of nearly 9,000 British children [75]. In comparison to children born to two nonobese parents, they report that children were 2.5 times more likely to be obese when they had an obese father, and 4.3 more likely to be obese if they had an obese mother. Further, children born to two obese parents were more than 10 times more likely to develop obesity by age 7 than those born to two non-obese parents [75]. It has been reported that gestational weight gain is also a predictor of childhood obesity, and that this impact is stronger in women who were obese prior to becoming pregnant [111]. Finally, recent reports suggest that surgical weight loss prior to pregnancy dramatically reduces the risk of childhood obesity in babies born to obese women [112]. These relationships suggest that any putative cause of the increasing prevalence of adult obesity [113] including those that are unlikely to play a direct role in the epidemic of childhood obesity (e.g-, iatrogenic weight gain [25]) may nonetheless play important indirect roles.

The relationship between parental and childhood obesity is likely to be linked via numerous mechanisms. For example, genetic factors are reported to account for roughly $25 \%$ of the variance in fat mass [114], which is likely to mediate some of the relationship in body composition between parent and child. Further, learned behavioural characteristics such as food choices, PA, and sedentary behaviours are also likely to mediate the transmission of intergenerational obesity [75, 115]. Finally, studies of animal models suggest that obesity or excessive weight gain during pregnancy is likely to predispose childhood obesity through deleterious changes in the central regulation of energy balance [116]. For example, lambs born to overfed ewes are less sensitive to signals of excess nutrient supply or fat mass than lambs born to control animals [116]. Taken together, the strong association between parental and childhood obesity and the numerous plausible mechanisms underlying these associations suggest that one of the most important drivers of the childhood obesity epidemic may in fact be adult obesity.

\section{Relative Contributions to Childhood Obesity}

As has been noted by others, there is currently insufficient information to make a truly objective ranking of the putative causes of an issue as complex as the current obesity epidemic [25]. However, the evidence presented above does allow some general conclusions to be made. This review has identified 4 factors-reduced sleep, increased sedentary time, increased consumption of sugar-sweetened beverages, and secular increases in adult obesity - which are likely to have made an important contribution to Canada's childhood obesity epidemic. Each of these factors has shown strong and consistent associations with childhood weight gain, has increased in prevalence during the obesity epidemic, and results in either biological or behavioural changes that are likely to promote positive energy balance. Of these, adult obesity appears to have the most powerful impact on childhood obesity levels, while reducing the consumption of sugar-sweetened beverages may be among the simplest ways to prevent future weight gain in individuals of all ages.

Available evidence provides only moderate support for the role of either total EI or PA in the etiology of childhood obesity. This is likely due to methodological limitations of self-reported intake and expenditure, as both of these factors are biologically plausible and have been shown to have impressive effects on adiposity in experimental studies. It is possible that methodological limitations may also explain the inconsistent relationships seen between obesity and dietary fat intake. Future studies employing more objective methods of measurement are important to determine the true role of these factors in the etiology of the childhood obesity epidemic.

Finally, although each has been linked in some way with childhood obesity, there is currently weak evidence supporting the role of maternal age, breastfeeding, exposure to endocrine disrupters, or calcium insufficiency in the etiology of the childhood obesity epidemic. Of these, maternal age, breastfeeding, and endocrine disruptors appear worthy of future study, while there is sufficient evidence to conclude that calcium intake plays little role in pediatric obesity rates at the population level.

\section{Summary}

Although influenced by numerous factors, available evidence suggests that the Canadian childhood obesity epidemic is most closely related to deleterious changes in sugarsweetened beverage intake, sedentary behaviour, reduced sleep, and adult obesity. Interventions aimed at modifying these factors may help to prevent further increases in obesity rates among the Canadian pediatric population.

\section{Acknowledgments}

The Author is supported by Doctoral Research Awards from the Canadian Institutes of Health Research and the Canadian Diabetes Association, as well as a University of Ottawa Excellence Scholarship. The funding agencies did not play a role in the decision to submit this paper for publication.

\section{References}

[1] I. Janssen, M. Shields, C. L. Craig, and M. S. Tremblay, Prevalence and secular changes in abdominal obesity in Canadian adolescents and adults, 1981 to 2007-2009," Obesity Reviews, vol. 12, no. 6, pp. 397-405, 2011.

[2] M. S. Tremblay, P. T. Katzmarzyk, and J. D. Willms, "Temporal trends in overweight and obesity in Canada, 1981-1996," 
International Journal of Obesity, vol. 26, no. 4, pp. 538-543, 2002.

[3] M. S. Tremblay and J. D. Willms, "Secular trends in the body mass index of Canadian children," CMAJ, vol. 163, no. 11, pp. $1429-1433,2000$

[4] M. S. Tremblay, M. Shields, M. Laviolette, C. L. Craig, I. Janssen, and S. C. Gorber, "Fitness of Canadian children and youth: results from the 2007-2009 Canadian Health Measures Survey," Health Reports, vol. 21, no. 1, pp. 7-20, 2010.

[5] S. Lee, F. Bacha, N. Gungor, and S. A. Arslanian, "Waist circumference is an independent predictor of insulin resistance in black and white youths," Journal of Pediatrics, vol. 148, no. 2, pp. 188-194, 2006.

[6] L. B. Andersen, L. Sardinha, K. Froberg, C. J. Riddoch, A. S. Page, and S. A. Anderssen, "Fitness, fatness and clustering of cardiovascular risk factors in children from Denmark, Estonia and Portugal: the European Youth Heart Study," International Journal of Pediatric Obesity, vol. 3, supplement International Journal

[7] W. H. Dietz, "Health consequences of obesity in youth: childhood predictors of adult disease," Pediatrics, vol. 101, no, 3, part 2, pp. 518-525, 1998 .

[8] D. S. Freedman, W. H. Dietz, S. R. Srinivasan, and G. S. Berenson, "The relation of overweight to cardiovascular risk factors among children and adolescents: the Bogalusa Heart Study," Pediatrics, vol. 103, no. 6, part 1, pp. 1175-1182, 1999.

[9] A. Must, P. F. Jacques, G. E. Dallal, C. J. Bajema, and W. H. Dietz, "Long-term morbidity and mortality of overweight adolescents - a follow-up of the Harvard Growth Study of 1922 to 1935," The New England Journal of Medicine, vol. 327, no. 19 , pp. 1350-1355, 1992.

[10] D. J. Gunnell, S. J. Frankel, K. Nanchahal, T. J. Peters, and G. D. Smith, "Childhood obesity and adult cardiovascular mortality: a 57-y follow-up study based on the Boyd Orr cohort," American Journal of Clinical Nutrition, vol. 67 , no. 6, pp. 1111-1118, 1998.

[11] E. Jéquier and L. Tappy, "Regulation of body weight in humans," Physiological Reviews, vol. 79, no. 2, pp. 451-480, 1999.

[12] A. Tremblay, E. Fontaine, E. T. Poehlman, D. Mitchell, L Perron, and C. Bouchard, "The effect of exercise-training on resting metabolic rate in lean and moderately obese individuals," International Journal of Obesity, vol. 10, no. 6, pp. 511-517, 1986.

[13] J. P. Chaput, L. Klingenberg, M. Rosenkilde, J. A. Gilbert, A. Tremblay, and A. Sjodin, "Physical activity plays an important role in body weight regulation," Journal of Obesity, vol. 2011, Article ID 360257, 11 pages, 2011.

[14] J. Mayer, P. Roy, and K. P. Mitra, "Relation between caloric intake, body weight, and physical work: studies in an industrial male population in West Bengal," American Journal of Clinical Nutrition, vol. 4, no. 2, pp. 169-175, 1956.

[15] R. J. Stubbs, D. A. Hughes, A. M. Johnstone, G. W. Horgan, N. King, and J. E. Blundell, "A decrease in physical activity affects appetite, energy, and nutrient balance in lean men feeding ad libitum," American Journal of Clinical Nutrition, vol. 79, no. 1, pp. 62-69, 2004.

[16] C. Martins, L. Morgan, and H. Truby, "A review of the effects of exercise on appetite regulation: an obesity perspective," International Journal of Obesity, vol. 32, no. 9, pp. 1337-1347, 2008.

[17] C. Martins, H. Truby, and L. M. Morgan, "Short-term appetite control in response to a 6-week exercise programme in sedentary volunteers," British Journal of Nutrition, vol. 98 , no. 4, pp. 834-842, 2007.

[18] A. Must and D. J. Tybor, "Physical activity and sedentary behavior: a review of longitudinal studies of weight and adiposity in youth," International Journal of Obesity, vol. 29. supplement 2, pp. S84-S96, 2005.

[19] M. M. Davis, B. Gance-Cleveland, S. Hassink, R. Johnson, G. Paradis, and K. Resnicow, "Recommendations for prevention of childhood obesity," Pediatrics, vol. 120, supplement 4, pp. S229-\$253, 2007 .

[20] W. B. Strong, R. M. Malina, C. J. R. Blimkie et al., "Evidence based physical activity for school-age youth," Journal of Pediatrics, vol. 146, no. 6, pp. 732-737, 2005.

[21] C. S. Berkey, H. R. Rockett, A. E. Field et al., "Activity, dietary intake, and weight changes in a longitudinal study of preadolescent and adolescent boys and girls," Pediatrics, vol. 105, no. 4, p. E56, 2000.

[22] J. B. Connelly, M. J. Duaso, and G. Butler, "A systematic review of controlled trials of interventions to prevent childhood obesity and overweight: a realistic synthesis of the evidence," Public Health, vol. 121, no. 7, pp. 510-517, 2007.

[23] M. S. Tremblay, D. W. Esliger, J. L. Copeland, J. D. Barnes, and D. R. Bassett, "Moving forward by looking back: lessons learned from long-lost lifestyles," Applied Physiology Nutrition and Metabolism, vol. 33, no. 4, pp. 836-842, 2008.

[24] S. A. Prince, K. B. Adamo, M. E. Hamel, J. Hardt, S. Connor Gorber, and M. Tremblay, "A comparison of direct versus self-report measures for assessing physical activity in adults: a systematic review," International Journal of Behavioral Nutrition and Physical Activity, vol. 5, no. 55, 2008.

[25] S. W. Keith, D. T. Redden, P. T. Katzmarzyk et al., "Putative contributors to the secular increase in obesity: exploring the roads less traveled," International Journal of Obesity, vol. 30, no. 11, pp. 1585-1594, 2006.

[26] J. C. Eisenmann, P. T. Katzmarzyk, and M. Tremblay, "Leisure-time physical activity levels among Canadian adolescents, 1981-1998," Journal of Physical Activity and Health, vol. 1, no. 2, pp. 154-162, 2004.

[27] R. N. Buliung, R. Mitra, and G. Faulkner, "Active school transportation in the Greater Toronto Area, Canada: an exploration of trends in space and time (1986-2006), Preventive Medicine, vol. 48, no. 6, pp. 507-512, 2009.

[28] D. R. Bassett, "Physical activity of Canadian and American children: a focus on youth in Amish, Mennonite, and modern cultures," Applied Physiology, Nutrition and Metabolism, vol. 33 , no. 4, pp. 831-835, 2008 .

[29] D. W. Esliger, M. S. Tremblay, J. L. Copeland, J. D. Barnes, G. E. Huntington, and D. R. Bassett Jr., "Physical activity profile of old order Amish, Mennonite, and contemporary children," Medicine and Science in Sports and Exercise, vol. 42, no. 2, pp. 296-303, 2010

[30] R. C. Colley, D. Garriguet, I. Janssen, C. L. Craig, J. Clarke, and M. S. Tremblay, "Physical activity of Canadian children and youth: accelerometer results from the 2007 to 2009 Canadian Health Measures Survey," Health Reports, vol. 22, no. 1, pp. 15-23, 2011.

[31] M. S. Tremblay, D. E. R. Warburton, I. Janssen et al., "New Canadian physical activityguidelines," Applied Physiolog! Nutrition and Metabolism, vol. 36, no. 1, pp. 36-46, 2011.

[32] M. S. Tremblay, D. E. R. Warburton, I. Janssen et al. "The new Canadian physical activity guidelines," Applied Physiology, Nutrition and Metabolism, vol. 36, no. 1, pp. 4758,2011 . 
[33] M. S. Tremblay, R. C. Colley, T. J. Saunders, G. N. Healy, and N. Owen, "Physiological and health implications of a sedentary lifestyle," Applied Physiology, Nutrition and Metabolism, vol. 35, no. 6, pp. 725-740, 2010.

[34] S. J. Marshall, T. Gorely, and S. J. H. Biddle, "A descriptive epidemiology of screen-based media use in youth: a review and critique," Journal of Adolescence, vol. 29, no. 3, pp. 333$349,2006$.

[35] M. C. Nelson, D. Neumark-Stzainer, P. J. Hannan, J. R. Sirard, and M. Story, "Longitudinal and secular trends in physical activity and sedentary behavior during adolescence," Pediatrics, vol. 118, no. 6, pp. e1627-e1634, 2006.

[36] Active Healthy Kids Canada, It's Time to Unplug Our KidsCanada's Report Card on Physical Activity for Children and Youth, Toronto, Ontario, Canada, 2008.

[37] S. J. Marshall, S. J. H. Biddle, T. Gorely, N. Cameron, and I. Murdey, "Relationships between media use, body fatness and physical activity in children and youth: a meta-analysis," International Journal of Obesity, vol. 28, no. 10, pp. 1238 1246, 2004.

[38] V. Burke, L. J. Beilin, K. Simmer et al., "Predictors of body mass index and associations with cardiovascular risk factors in Australian children: a prospective cohort study, International Journal of Obesity, vol. 29, no. 1, pp. 15-23, 2005.

[39] T. N. Robinson, "Reducing children's television viewing to prevent obesity: a randomized controlled trial," Journal of the American Medical Association, vol. 282, no. 16, pp. 1561$1567,1999$.

[40] Active Healthy Kids Canada, Healthy Habits Start Earliet Than You Think-Canada's Report Card on Physical Activity for Children and Youth, Toronto, Ontario, Canada, 2010.

[41] U. Ekelund, S. Brage, K. Froberg et al., "TV viewing and physical activity are independently associated with metabolic risk in children: the European Youth Heart Study," PLoS Medicine, vol. 3, no. 12, article e488, pp. 2449-2457, 2006.

[42] J. P. Rey-López, G. Vicente-Rodriguez, M. Biosca, and L. A. Moreno, "Sedentary behaviour and obesity developmen in children and adolescents," Nutrition, Metabolism and Cardiovascular Diseases, vol. 18, no. 3, pp. 242-251, 2008.

[43] J. L. Wiecha, K. E. Peterson, D. S. Ludwig, J. Kim, A. Sobol, and S. L. Gortmaker, "When children eat what they watch: impact of television viewing on dietary intake in youth," Archives of Pediatrics and Adolescent Medicine, vol. 160, no. 4, pp. 436-442, 2006.

[44] J. C. G. Halford, E. J. Boyland, G. Hughes, L. P. Oliveira, and T. M. Dovey, "Beyond-brand effect of television (TV) food advertisements/commercials on caloric intake and food choice of 5-7-year-old children," Appetite, vol. 49, no. 1, pp. 263-267, 2007.

[45] J. C. G. Halford, J. Gillespie, V. Brown, E. E. Pontin, and T. M. Dovey, "Effect of television advertisements for foods on food consumption in children," Appetite, vol. 42, no. 2, pp. 221-225, 2004

[46] C. Bouchard, A. Tremblay, J. P. Despres et al., "The response to long-term overfeeding in identical twins," The New England Journal of Medicine, vol. 322, no. 21, pp. 1477-1482, 1990.

[47] J. A. Levine, N. L. Eberhardt, and M. D. Jensen, "Role of nonexercise activity thermogenesis in resistance to fat gain in humans," Science, vol. 283, no. 5399, pp. 212-214, 1999.

[48] N. G. Norgan and J. V. G. A. Durnin, "The effect of 6 weeks of overfeeding on the body weight, body composition, and energy metabolism of young men," American Journal of Clinical Nutrition, vol. 33, no. 5, pp. 978-988, 1980.

[49] L. A. Moreno and G. Rodriguez, "Dietary risk factors for development of childhood obesity," Current Opinion in Clinical Nutrition and Metabolic Care, vol. 10, no. 3, pp. 336341, 2007.

[50] J. Pomerleau, T. Østbye, and E. Bright-See, "Potential underreporting of energy intake in the Ontario Health Survey and its relationship with nutrient and food intakes," European Journal of Epidemiology, vol. 15, no. 6, pp. 553-557, 1999.

[51] C. M. Champagne, N. B. Baker, J. P. DeLany, D. W. Harsha, and G. A. Bray, "Assessment of energy intake underreporting by doubly labeled water and observations on reported nutrient intakes in children," Journal of the American Dietetic Association, vol. 98, no. 4, pp. 426-430, 1998.

[52] T. T. K. Huang, N. C. Howarth, B. H. Lin, S. B. Roberts, and M. A. McCrory, "Energy intake and meal portions: associations with BMI percentile in U.S. children," Obesity Research, vol. 12, no. 11, pp. 1875-1885, 2004.

[53] S. J. Nielsen, A. M. Siega-Riz, and B. M. Popkin, "'Trends in energy intake in U.S. between 1977 and 1996: similar shifts seen across age groups," Obesity Research, vol. 10, no. 5, pp. 370-378, 2002.

[54] Y. K. Park and E. A. Yetley, "Intakes and food sources of fructose in the United States," American Journal of Clinical Nutrition, vol. 58, no. 5, pp. 737S-747S, 1993.

[55] B. P. Marriott, N. Cole, and E. Lee, "National estimates of dietary fructose intake increased from 1977 to 2004 in the United States," Journal of Nutrition, vol. 139, no. 6, pp. $1228 \mathrm{~S}-1235 \mathrm{~S}, 2009$.

[56] S. J. Nielsen and B. M. Popkin, "Patterns and trends in food portion sizes, 1977-1998," Journal of the American Medical Association, vol. 289, no. 4, pp. 450-453, 2003.

[57] R. P. Troiano, R. R. Briefel, M. D. Carroll, and K. Bialostosky, "Energy and fat intakes of children arid adolescents in the United States: data from the National Health and Nutrition Examination Surveys," American Journal of Clinical Nutrition, vol. 72, no. 5, pp. 1343S-1353S, 2000.

[58] C. Cavadini, A. M. Siega-Riz, and B. M. Popkin, "US adolescent food intake trends from 1965 to $1996, "$ Archives of Disease in Childhood, vol. 83, no. 1, pp. 18-24, 2000.

[59] S. A. French, B. H. Lin, and J. F. Guthrie, "National trends in soft drink consumption among children and adolescents age 6 to 17 years: prevalence, amounts, and sources, $1977 / 1978$ to 1994/1998," Journal of the American Dietetic Association, vol. 103, no. 10, pp. 1326-1331, 2003 .

[60] Y. C. Wang, S. N. Bleich, and S. L. Gortmaker, "Increasing caloric contribution from sugar- sweetened beverages and $100 \%$ fruit juices among US children and adolescents, 19882004," Pediatrics, vol. 121, no. 6, pp. e1604-e1614, 2008.

[61] V. S. Malik, M. B. Schulze, and F. B. Hu, "Intake of sugarsweetened beverages and weight gain: a systematic review," American Journal of Clinical Nutrition, vol. 84, no. 2, pp. 274288, 2006.

[62] L. R. Vartanian, M. B. Schwartz, and K. D. Brownell, "Effects of soft drink consumption on nutrition and health: a systematic review and meta-analysis," American Journal of Public Health, vol. 97, no. 4, pp. 667-675, 2007.

[63] D. S. Ludwig, K. E. Peterson, and S. L. Gortmaker, "Relation between consumption of sugar-sweetened drinks and childhood obesity: a prospective, observational analysis," The Lancet, wol. 357, no. 9255, pp. 505-508, 2001. 
[64] Y. C. Wang, D. S. Ludwig, K. Sonneville, and S. L. Gortmaker, "Impact of change in sweetened caloric beverage consumption on energy intake among children and adolescents," Archives of Pediatrics and Adolescent Medicine, vol. 163, no. 4, pp. 336-343, 2009.

[65] K. L. Stanhope and P. J. Havel, "Endocrine and metabolic effects of consuming beverages sweetened with fructose, glucose, sucrose, or high-fructose corn syrup," American Journal of Clinical Nutrition, vol. 88, no. 6, pp. 1733S-1737S, 2008.

[66] J. E. Blundell and J. I. Macdiarmid, "Fat as a risk factor for overconsumption: satiation, satiety, and patterns of eating," Journal of the American Dietetic Association, vol. 97, no. 7, pp. S63-S69, 1997

[67] G. A. Bray and B. M. Popkin, "Dietary fat intake does affect obesity," American Journal of Clinical Nutrition, vol. 68, no, 6, pp. 1157-1173, 1998.

[68] S. J. Nielsen and B. M. Popkin, "Changes in beverage intake between 1977 and 2001," American Journal of Preventive Medicine, vol. 27, no, 3, pp, 205-210, 2004.

[69] A. Tremblay and J. P. Chaput, "About unsuspected potential determinants of obesity," Applied Physiology, Nutrition and Metabolism, vol. 33, no. 4, pp. 791-796, 2008.

[70] G. C. Major, J. P. Chaput, M. Ledoux et al,, "Recent developments in calcium-related obesity research," Obesity Reviews, vol. 9, no. 5, pp. 428-445, 2008.

[71] T. Winzenberg, K. Shaw, J. Fryer, and G. Jones, "Calcium supplements in healthy children do not affect weight gain, height, or body composition," Obesity, vol. 15, no. 7, pp. 1789-1798, 2007

[72] R. Trowman, J. C. Dumville, S. Hahn, and D. J. Torgerson, "A systematic review of the effects of calcium supplementation on body weight," British Journal of Nutrition, vol. 95, no. 6, pp. 1033-1038, 2006.

[73] F. P. Cappuccio, F. M. Taggart, N. B. Kandala et al., "Metaanalysis of short sleep duration and obesity in children and adults," Sleep, vol. 31, no. 5, pp. 619-626, 2008.

[74] T. Young, "Increasing sleep duration for a healthier (and less obese?) population tomorrow," Sleep, vol. 31, no. 5, pp. 593$594,2008$.

[75] J. J. Reilly, J. Armstrong, A. R. Dorosty et al., "Early life risk factors for obesity in childhood: cohort study," British Medical Journal, vol. 330, no. 7504, pp. 1357-1359, 2005.

[76] J. F. Bell and F. J. Zimmerman, "Shortened nighttime sleep duration in early life and subsequent childhood obesity," Archives of Pediatrics and Adolescent Medicine, vol. 164, no.

[77] I. Iglowstein, O. G. Jenni, L. Molinari, and R. H. Largo, "Sleep duration from infancy to adolescence: reference values and generational trends," Pediatrics, vol. 111, no. 2, pp. 302-307, 2003.

[78] J. Dollman, K. Ridley, T. Olds, and E. Lowe, "Trends in the duration of school-day sleep among 10- to 15-year-old South Australians between 1985 and 2004," Acta Paediatrica, vol. 96, no. 7, pp. 1011-1014, 2007.

[79] S. Pallesen, J. Hetland, B. Sivertsen, O. Samdal, T. Torsheim, and H. I. Nordhus, "Time trends in sleep-onset difficulties among Norwegian adolescents: 1983-2005," Scandinavian Journal of Public Health, vol. 36, no. 8, pp. 889-895, 2008

[80] C. A. Magee, X. F. Huang, D. C. Iverson, and P. Caputi, "Examining the pathways linking chronic sleep restriction to obesity," Journal of Obesity, vol. 2010, Article ID 821710, 8 pages, 2010.
[81] K. L. Knutson, K. Spiegel, P. Peney, and E. van Cauter, "The metabolic consequences of sleep deprivation," Sleep Medicine Reviews, vol. 11, no. 3, pp. 163-178, 2007.

[82] K. Spiegel, R. Leproult, M. L'Hermite-Balériaux, G. Copinschi, P. D. Penev, and E. van Cauter, "Leptin levels are dependent on sleep duration: relationships with sympathovagal balance, carbohydrate regulation, cortisol, and thyrotropin," Journal of Clinical Endocrinology and Metabolism, vol. 89, no. 11, pp. 5762-5771, 2004.

[83] K. Spiegel, R. Leproult, and E. van Cauter, "Impact of sleep debt on metabolic and endocrine function," The Lancet, vol. 354 , no. 9188 , pp. $1435-1439,1999$.

[84] K. Spiegel, E. Tasali, P. Penev, and E. van Cauter, "Brief communication: sleep curtailment in healthy young men is associated with decreased leptin levels, elevated ghrelin levels, and increased hunger and appetite," Annals of Internal Medicine, vol. 141, no. 11, pp. 846-850, 2004

[85] C. M. Novak and J. A. Levine, "Central neural and endocrine mechanisms of non-exercise activity thermogenesis and their potential impact on obesity," Journal of Neuroendocrinology, vol. 19, no. 12, pp. 923-940, 2007.

[86] L. Brondel, M. A. Romer, P. M. Nougues, P. Touyarou, and D. Davenne, "Acute partial sleep deprivation increases food intake in healthy men," American Journal of Clinical Nutrition, vol. 91, no, 6, pp. 1550-1559, 2010.

[87] E. Diamanti-Kandarakis, J. P. Bourguignon, L. C. Giudice et al., "Endocrine-disrupting chemicals: an endocrine society scientific statement," Endocrine Reviews, vol. 30, no. 4, pp. 293-342, 2009.

[88] F. Grün and B. Blumberg, "Environmental obesogens: organotins and endocrine disruption via nuclear recepto signaling," Endocrinology, vol. 147, no. 6, pp. S50-S55, 2006.

[89] C. Koopman-Esseboom, D. C. Morse, N. Weisglas-Kuperus et al., "Effects of dioxins and polychlorinated biphenyls on thyroid hormone status of pregnant women and their infants," Pediatric Research, vol. 36, no. 4, pp. 468-473, 1994.

[90] C. Pelletier, E. Doucet, P. Imbeault, and A. Tremblay, "Association between weight loss-induced changes in plasma organochlorine concentrations, serum $\mathrm{T}(3)$ concentration, and resting metabolic rate," Toxicological Sciences, vol. 67, no. 1, pp. 46-51, 2002.

[91] P. Imbeault, A. Tremblay, J. A. Simoneau, and D. R. Joanisse, "Weight loss-induced rise in plasma pollutant is associated with reduced skeletal muscle oxidative capacity," American Journal of Physiology - Endocrinology and Metabolism, vol. 282, no. 3, Pp. E574-E579, 2002.

[92] E. E. Hatch, I, W. Nelson, R. W. Stahlhut, and T. F. Webster, "Association of endocrine disruptors and obesity: perspectives from epidemiological studies," International Journal of Andrology, vol. 33, no. 2, pp. 324-331, 2010.

[93] W. Karmaus, J. R. Osuch, I. Eneli et al., "Maternal levels of dichlorodiphenyl-dichloroethylene (DDE) may increase weight and body mass index in adult female offspring," Occupational and Environmental Medicine, vol. 66, no. 3, pp. 143-149, 2009.

[94] H. M. Blanck, M. Marcus, C. Rubin et al., "Growth in girls exposed in utero and postnatally to polybrominated biphenyls and polychlorinated biphenyls," Epidemiology, vol. 13 , no. 2, pp. 205-210, 2002.

[95] S. Loh and B. Ram, "Delayed childbearing in Canada: trends and factors," Genus, vol. 46, no, 1-2, pp. 147-161, 1990,

[96] S. C. Tough, C. Newburn-Cook, D. W. Johnston, L. W. Svenson, S. Rose, and J. Belik, "Delayed childbearing and 
its impact on population rate changes in lower birth weight, multiple birth, and preterm delivery," Pediatrics, vol. 109, no. 3 , pp. 399-403, 2002.

[97] S. Wadhera, "Trends in birth and fertility rates, Canada, 1921-1987," Health Reports, vol. 1, no. 2, pp. 211-223, 1989.

[98] S. J. Ventura, "First births to older mothers, 1970-86," American Journal of Public Health, vol. 79, no. 12, pp. 1675-1677, 1989.

[99] G. Breart, "Delayed childbearing," European Journal of Obstetrics Gynecology and Reproductive Biology, vol. 75, no. 1, pp. 71-73, 1997.

[100] U. Kalberer, D. Baud, A. Fontanet, P. Hohlfeld, and D. de Ziegler, "Birth records from Swiss married couples analyzed over the past 35 years reveal an aging of first-time mothers by 5.1 years while the interpregnancy interval has shortened," Fertility and Sterility, vol. 92, no. 6, pp. 2072-2073, 2009.

[101] K. K. L. Ong, M. L. Ahmed, P. M. Emmett, M. A. Preece, and D, B. Dunger, "Association between postnatal catch-up growth and obesity in childhood: prospective cohort study," British Medical Journal, vol. 320, no. 7240, pp. 967-971, 2000.

[102] O. Hue, J. Marcotte, F. Berrigan et al., "Plasma concentration of organochlorine compounds is associated with age and not obesity," Chemosphere, vol. 67, no. 7, pp. 1463-1467, 2007.

[103] J. L. Kuk, T. J. Saunders, L. E. Davidson, and R. Ross, "Agerelated changes in total and regional fat distribution," Ageing Research Reviews, vol. 8, no. 4, pp. 339-348, 2009.

[104] A. Smink, N. Ribas-Fito, R. Garcia et al., "Exposure to hexachlorobenzene during pregnancy increases the risk of overweight in children aged 6 years," Acta Paediatrica, vol. 97, no. 10, pp. 1465-1469, 2008.

[105] M. E. Symonds, S. Pearce, J. Bispham, D. S. Gardner, and T. Stephenson, "Timing of nutrient restriction and programming of fetal adipose tissue development," Proceedings of the Nutrition Saciety, vol. 63, no. 3, pp. 397-403, 2004.

[106] M. L. Patterson, S. Stern, P. B. Crawford et al., "Sociodemographic factors and obesity in preadolescent black and white girls: NHLBI's Growth and Health Study," Journal of the National Medical Association, vol. 89, no. 9, pp. 594-600, 1997.

[107] T. Harder, R. Bergmann, G. Kallischnigg, and A. Plagemann, "Duration of breastfeeding and risk of overweight: a metaanalysis," American Journal of Epidermiology, vol. 162, no. 5, pp. 397-403, 2005.

[108] A. Yngve and M. Sjōstrŏm, "Breastfeeding in countries of the European Union and EFTA: current and proposed recommendations, rationale, prevalence, duration and trends," Public Health Nutrition, vol. 4, no. 2, pp. 631-645, 2001.

[109] A. S. Ryan, Z. Wenjun, and A. Acosta, "Breastfeeding continues to increase into the new millennium," Pediatrics, vol. 110 , no. 6, pp. 1103-1109, 2002

[110] E. Oken, E. M. Taveras, K. P. Kleinman, J. W. Rich-Edwards, and M. W. Gillman, "Gestational weight gain and child adiposity at age 3 years," American Journal of Obstetrics and Gynecology, vol. 196, no. 4, pp. 322.el-322.e8, 2007.

[111] C. M. Olson, M. S. Strawderman, and B. A. Dennison, "Maternal weight gain during pregnancy and child weight at age 3 years," Maternal and Child Health Journal, vol. 13, no. 6, pp. $839-846,2009$.

[112] J. G. Kral, S. Biron, S. Simard et al., "Large maternal weight loss from obesity surgery prevents transmission of obesity to children who were followed for 2 to 18 years," Pediatrics, vol. 118 , no. 6, pp. el644-e1649, 2006.

[113] M. Shields, M. S. Tremblay, M. Laviolette, C. L. Craig, I. Janssen, and S. C. Gorber, "Fitness of Canadian adults: results from the 2007-2009 Canadian Health Measures Survey," Health Reports, vol. 21, no. 1, pp. 21-35, 2010.

114] C. Bouchard, "Current understanding of the etiology of obesity: genetic and nongenetic factors," American Journal of Clinical Nutrition, vol. 53, no. 6, pp. 1561S-1565S, 1991.

[115] L. A. Francis, Y. Lee, and L. L. Birch, "Parental weight status and girls' television viewing, snacking, and body mass indexes," Obesity Research, vol. 11, no. 1, pp. 143-151, 2003.

[116] I. C. McMillen, L. Rattanatray, J. A. Duffield et al., "The early origins of later obesity: pathways and mechanisms," Advances in Experimental Medicine and Biology, vol. 646, pp. 71-81, 2009. 


\title{
Systematic review of sedentary behaviour and health indicators in school-aged children and youth
}

\author{
Mark S Tremblay ${ }^{1 *}$, Allana G LeBlanc ${ }^{1}$, Michelle E $1 \mathrm{Kho}^{2}$, Travis J Saunders', Richard Larouche', Rachel C Colley',
} Gary Goldfield ${ }^{1}$ and Sarah Connor Gorber ${ }^{3}$

\begin{abstract}
Accumulating evidence suggests that, independent of physical activity levels, sedentary behaviours are assoclated with increased risk of cardio-metabolic disease, all-cause mortality, and a variety of physiological and psychological problems. Therefore, the purpose of this systematic review is to determine the relationship between sedentary behaviour and health indicators in school-aged children and youth aged 5-17 years. Online databases SMEDLINE, EMBASE and PsycINFO), personal libraries and government documents were searched for relevant studies examining time spent engaging in sedentary behaviours and six specific health indicators (body composition, fitness, metabolic syndrome and cardiovascular disease, self-esteem, pro-social behaviour and academic achievement). 232 studies including 983,840 participants met inclusion criteria and were included in the review. Television (TV) watching was the most common measure of sedentary behaviour and body composition was the most common outcome measure. Cualitative analysis of all studies revealed a dose-response relation between increased sedentary behaviour and unfavourable health outcomes. Watching TV for more than 2 hours per day was associated with unfavourable body composition, decreased fitness, lowered scores for self-esteem and pro-social behaviour and decreased academic achievement. Meta-analysis was completed for randomized controlled studies that aimed to reduce sedentary time and reported change in body mass index (BMI) as their primary outcome. In this regard, a metaanalysis revealed an overall significant effect of $-0.81(95 \% \mathrm{Cl}$ of -1.44 to $-0.17, \mathrm{p}=0.01)$ indicating an overall decrease in mean BMI associated with the interventions. There is a large body of evidence from all study designs which suggests that decreasing any type of sedentary time is asscciated with lower health risk in youth aged 5-17 years. In particular, the evidence suggests that daily TV viewing in excess of 2 hours is associated with reduced physical and psychosocial health, and that lowering sedentary time leads to reductions in BMI.
\end{abstract}

Keywords: Inactivity, sitting, TV, body composition, fitness, metabolic syndrome, cardiovascular disease, self-esteem, pro-social behaviour, academic achievement

\section{Introduction}

Engaging in regular physical activity is widely accepted as an effective preventative measure for a variety of health risk factors across all age, gender, ethnic and socioeconomic subgroups [1-6]. However, across all age groups, levels of physical activity remain low [7-12] and obesity rates continue to rise $[10,11,13,14]$; collectively threatening the persistent increase in life expectancy enjoyed over the past century and efforts to counteract the inactivity and obesity crisis [15].

This inactivity crisis is especially important in the pediatric population as recent data from the Canadian Health Measures Survey [8] suggest that only $7 \%$ of children and youth aged 6-19 years participate in at least 60 minutes of moderate- to vigorous-intensity physical activity per day, thus meeting the current physical activity guidelines from Canada [16], the U.S. [6], the U.K [17], Australia [18] and the World Health Organization (W/HO) [5]. However, even for those children and youth who meet current guidelines, there remains 23 hours per day for school, sleep, work, and discretionary time. Several sources report that children and youth spend the majority of their
() Biomed Central

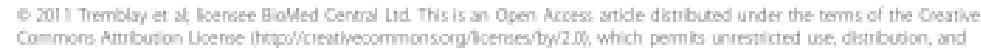
reproduction in ary medium, prowided the orignil work is propety cted. 
discretionary time engaging in sedentary pursuits (e.g. watching television (TV) or playing video games) [8,19-28]. Canadian children and youth are spending an average of 8.6 hours per day, or $62 \%$ of their waking hours being sedentary [8]. Similar trends are being reported in the US. where children and youth spend an average of 6-8 hours per day being sedentary [22-28]. Accumulating evidence shows that, independent of physical activity levels, sedentary behaviours are associated with increased risk of cardio-metabolic disease, all-cause mortality, and a variety of physiological and psychological problems [29-31]. Therefore, to maximize health benefits, approaches to resolve the inactivity crisis should attempt to both increase deliberate physical activity and decrease sedentary behaviours, especially in the pediatric population. However, to date, public health efforts have focused primarily on physical activity and have paid little attention to the mounting evidence to support sedentary behaviour as a distinct behaviour related to poor health.

A recent scoping review identified review articles, meta-analyses, and grey literature that examined the relationship between sedentary behaviour and health [32], The large majority of this information reported on the relationship between screen time and body composition and did not include other indicators of health [23-25]. Furthermore, none of these reviews followed the rigorous process of a systematic review and are therefore not able to be used to inform the development of clinical practice guidelines. As a result, to our knowledge, there are no systematic, evidence-based sedentary behaviour guidelines for any age group, anywhere in the world. Guidelines that do exist are largely based on expert opinion or narrative literature reviews $[33,34]$.

Therefore, the purpose of this systematic review was to gather, catalog, assess and evaluate the available evidence examining sedentary behaviours in relation to selected health outcomes in children and youth 5-17 years of age and present a summary of the best available evidence. Specifically, the review presents available evidence for minimal and optimal thresholds for daily sedentary time in children and youth, and when possible, how thresholds differ across health outcome or demographic status (i.e. age, gender). The information gathered in this review can serve to guide future research and inform the development of evidence-based clinical practice guideline recommendations for safe and healthy amounts of daily sedentary behaviour in the pediatric population.

\section{Methods}

\section{Study Inclusion Criteria}

The review sought to identify all studies that examined the relationship between sedentary behaviour and a specific health outcome in children and youth (aged 5-17 years).
All study designs were eligible (e.g. cross sectional, retrospective, prospective, case control, randomized controlled trial (RCT), longitudinal), Longitudinal studies were included if the data presented in the article was consistent with the age limits that were set (i.e. if the study looked at participants at age 10 and then again at age 30 , only baseline measurements from age 10 were used).

Studies were included only if there was a specific measure of sedentary behaviour. Eligible exposures of sedentary behaviours included those obtained via direct (e.g. measurements of sitting, or low activity measured by accelerometer) and self-reported (e.g., questionnaires asking about TV watching, video gaming, non-school computer use, and screen time - composite measures of TV, video games, computers) methods. Sedentary behaviour was often measured as a composite measure of all time engaging in sedentary behaviours including screen time outside of school hours. Six health indicators were chosen based on the literature, expert input, and a desire to have relevant measures from a range of holistic health indicators (L.e. not only physical health, but also emotional, mental and intellectual health). The six eligible indicators in this review were:

1. Body composition (overweight/obesity measured by body mass index (BMI), waist circumference, skin folds, bio-impedance analysis (BLA), dual-energy $x$ ray absorptiometry (DXA or DEXA));

2. Fitness (physical fitness, physical conditioning, musculoskeletal fitness, cardiovascular fitness);

3. Metabolic syndrome (MS) and cardiovascular disease (CVD) risk factors (unfavourable lipid levels, blood pressure, markers for insulin resistance or type 2 diabetes);

4. Self-esteem (self-concept, self-esteem, self efficacy);

5. Behavioural conduct/pro-social behaviour (child behaviour disorders, child development disorder, prosocial behaviour, behavioural conduct, aggression); 6. Academic achievement (school performance, grade-point average).

No Language or date limits were imposed in the search. The following definitions were used to help guide the systematic review [31]:

- Sedentary: A distinct class of behaviours (e.g. sitting. watching TV, playing video games) characterized by little physical movement and low energy expenditure ( 51.5 METs).

- Sedentarism: Engagement in sedentary behaviours characterized by minimal movement, low energy expenditure, and rest. 
- Physically active: Meeting established physical activity guidelines (e.g. see Tremblay et aL 2011 for Canadian Physical Activity Guidelines [16]\}.

- Prysical inactivity: The absence of physical activity, usually reflected as the proportion of time not engaged in physical activity of a pre-determined intensity and therefore not meeting established physical activity guidelines.

\section{Study Exclusion Criteria}

As the volume of literature on sedentary behaviour was anticipated to be very high, to control the feasibility of this project, the following sample size limits were set $a$. priori: population based studies (observational, cross sectional, cohort, and retrospective studies) were required to have a minimum sample size of 300 participants; RCTs, and intervention studies were required to have at least 30 participants. Studies of 'active gaming' (e.g., Nintendo WiiTM, Microsoft Kinect TM, Sony's Playstation Move TM, video arcades, etc.) were excluded. Finally, studies that defined sedentary behaviour as 'failing to meet physical activity guidelines' were excluded from the review.

\section{Search strategy}

The following electronic bibliographic databases were searched using a comprehensive search strategy to identify relevant studies: Ovid MEDLINE(R) (1950 to February Week 22010 ), Ovid EMBASE (1980 to 2010 Week 07), and Ovid psycINFO (1806 to February Week 3 2010 ). The search strategy was created by a single researcher (JM) and run by a second researcher ( $A L$ ). The search strategies can be found in Additional file 1. The search was limited to studies looking at 'school-aged' children and youth (mean age of 5-17 years). Articles were extracted as text files from the OVID interface and imported in to Reference Manager Software (Thompson Reuters, San Francisco, CA). Duplicate articles were first removed using Reference Manager Software, and any remaining duplicates were removed manually. All articles were given a unique reference identification number in the database.

Titles and abstracts of potentially relevant articles were screened by two reviewers (AL and one of GG, MT, RC, RL or TS) and full text coples were obtained for all articles meeting initial screening by at least one reviewer. Two independent reviewers examined all full text articles (AL and one of GG, MT, RC, RL. or TS) and any discrepancies were resolved by discussion and consensus between the two reviewers. If the reviewers were unable to reach consensus, a third reviewer was asked to look at the article in question. Consensus was obtained for all included articles
Twelve key content experts were contacted and asked to identify the most influential papers from their personal libraries examining sedentary behaviour and health in the pediatric age group. Government documents from the U.S [6], the U.K. [17], and Australia [18] were used for reference and to help guide the review process.

\section{Data extraction}

Standardized data extraction tables were created; data extraction was completed by one reviewer (AL) and checked by another (one of GG, RC, RL, or TS) for accuracy. Information was extracted regarding study characteristics (i.e. year, study design, country, number of participants, age), type of sedentary behaviour, measure of sedentary behaviour (i.e. direct, or indirect), and health outcome. Reviewers were not blinded to the authors or journals when extracting data.

\section{Risk of bias assessment}

The Downs and Black checklist was used to asses study quality [35]. This 27 point checklist assesses the quality of reporting (e,g. "Are the main findings of the study clearly described"); external validity (e.g. "Were the subjects asked to participate representative of the entire population from which they were recruited"); internal validity (e.g. "Were subjects randomized to intervention groups"); and power (e.g, "Was there sufficient power such that the difference being due to chance is less than $5 \%$ ). The maximum score a study can receive is 32 , with higher scores indicating better quality. Inter-rater reliability was calculated using Cohen's kappa.

Quality of evidence was determined by the study design and by Downs and Black score. Level of evidence was used to explain the quality of available studies and the confidence of the findings [36]. RCTs were considered to have the highest level of evidence while anecdotal reports were considered to have the lowest evidence See Table 1 for more details. When possible, studies were examined for differences among age and gender subgroups.

\section{Analysis}

A meta-analysis was performed with the data that were sufficiently homogeneous in terms of statistical, clinical, and methodological characteristics using Review Manager Software 5.0 (The Cochrane Collaboration, Copenhagen Denmark). Pooled estimates for the meta-analysis and their $95 \%$ confidence intervals were obtained using the random effects estimator of DerSimonian-Laird [37]. Studies were weighted by the inverse of their variance. Cochrane's $Q$ was used to test for heterogeneity among studies and the $\mathrm{I}^{2}$ (squared) index [10] was used to determine the degree of heterogeneity [38]. Funnel plots were 
Table 1 Criteria for assigning level of evidence to a recommendation

\begin{tabular}{|c|c|}
\hline Level of evidence & Criteria \\
\hline Level 1 & - Plandomized control trials without importank limitations \\
\hline Level 2 & $\begin{array}{l}\text { - Pondomized control trials with important limitations } \\
\text { - Observational studies [non-randomized dinical trials of cohort studies) with overwhelming evidence }\end{array}$ \\
\hline Level 3 & - Cther obsenational studies (prospective cohort studies, case-control studies, case series) \\
\hline Level 4 & $\begin{array}{l}\text { - Inadequate or no data in population of interest } \\
\text { - Anecdotal evidence or clinical experience }\end{array}$ \\
\hline
\end{tabular}

Adsapted from: Lau OC et a. 2005 [30]

used to assess publication bias (data not shown). Qualitative syntheses were conducted for remaining studies.

\section{Results}

Description of studies

After de-duplication, the preliminary search of electronic databases, reference lists, and grey literature identified 5,291 potentially relevant articles (Figure 1). Of these, 3,299 were identified in MEDLINE, 1,016 in EMBASE, 912 in psyclNFO, and 64 through key informants, government documents, and bibliographies. After a preliminary review of titles and abstracts, 828 articles were included for detailed assessment of the full text article. Of these, 232 met the criteria for study inclusion ( 8 RCTs, 10 intervention studies, 37 longitudinal studies and 177 cross sectional studies). Individual study charac teristics can be seen in Table 2. Reasons for excluding studies included: ineligible population (e.g. ineligible age or sample size) ( $n=161$ ), ineligible exposure (e.g. diet, physical activity) ( $n=145$ ), ineligible measure of sedentary behaviour (i.e. not meeting physical activity guidelines) ( $n=19$ ), ineligible outcome ( $n=60$ ), ineligible analysis (e.g. analysis focused on content of screen time versus duration of screen time, analysis focused on active video gaming) ( $n=60$ ), and 'other' ( $n=216$ ) (e.g. commentary article or methodological paper). Some studies were excluded for multiple reasons. Some articles ( $n=9$ ) could not be retrieved due to missing or incorrect reference information.

Table 2 provides a summary of all studies included in the review. The majority of the studies included in this systematic review were cross sectional $(n=177)$. In total, data from 983,840 participants were included in this review. Studies ranged from 30 participants in intervention studies and RCTs, to 62,876 participants in cross sectional observational investigations. Articles were pub. lished over a 51 year period from 1958 to 2009 , and included participants ranging from 2-19 years of age. Although the scope of the review focused on those 5-17 years of age, studies that had a range below 5 years or over 17 years were not excluded as long as the mean age was between $5-17$ years. Included studies involved participants from 39 countries; there were a greater number of articles reporting on female-only data than those reporting on male-only data. Translators were contracted to read non-English articles and complete any necessary data extraction for studies that met inclusion criteria ( $\mathrm{n}=8$ ).

Of the 232 studies, 170 studies reported data on body composition, 15 on fitness, 11 on MS and CVD, 14 on self-esteem, 18 on pro-social behaviour, and 35 on academic achievement. The majority of studies ( $n=223$ ) used indirect measures to assess sedentary behaviour (i.e. parent-, teacher-, or self-report questionnaires). There were 14 studies $[24,27,28,39-49]$ that directly measured sedentary behaviour with accelerometers and one that directly measured television viewing through a monitoring device [50]. The direction of the association between increased sedentary behaviour and health outcomes were similar between direct and indirect measures. Meta-analysis was conducted for RCTs examining change in body mass index.

\section{Risk of bias assessment}

Risk of bias assessment was completed for all included studies (Additional file 2). The mean Downs and Black score was 20.7 (range $=16-26$ ). The studies were then split into groups and labeled as 'high quality' (score 23$26, \mathrm{n}=36$ ), 'moderate quality' (score 19-22, $\mathrm{n}=169$ ), and 'lower quality' (score 16-18, $\mathrm{n}=27$ ). Quality of study did not affect the outcome of the study; in other words, both lower quality and high quality studies showed a positive relationship between increased time spent sedentary and health risk. Inter-reviewer assessment using the Downs and Black tool was very high (kappa $=0.98$ ).

\section{Data Synthesis}

Body composition

Of the 232 studies included in this review, 170 examined body composition, with the majority of these focusing on the relationship between overweight and obesity and time spent watching TV (Table 3). Body composition was measured in a variety of ways including body mass index (BMI), sum of skin folds, percent body fat and various composite measures (e.g. BMI + sum of skin folds). Of the 8 RCTs, 7 showed that decreases in sedentary time lead to reductions in body weight (see meta-analysis below for details). Intervention studies reported desirable 


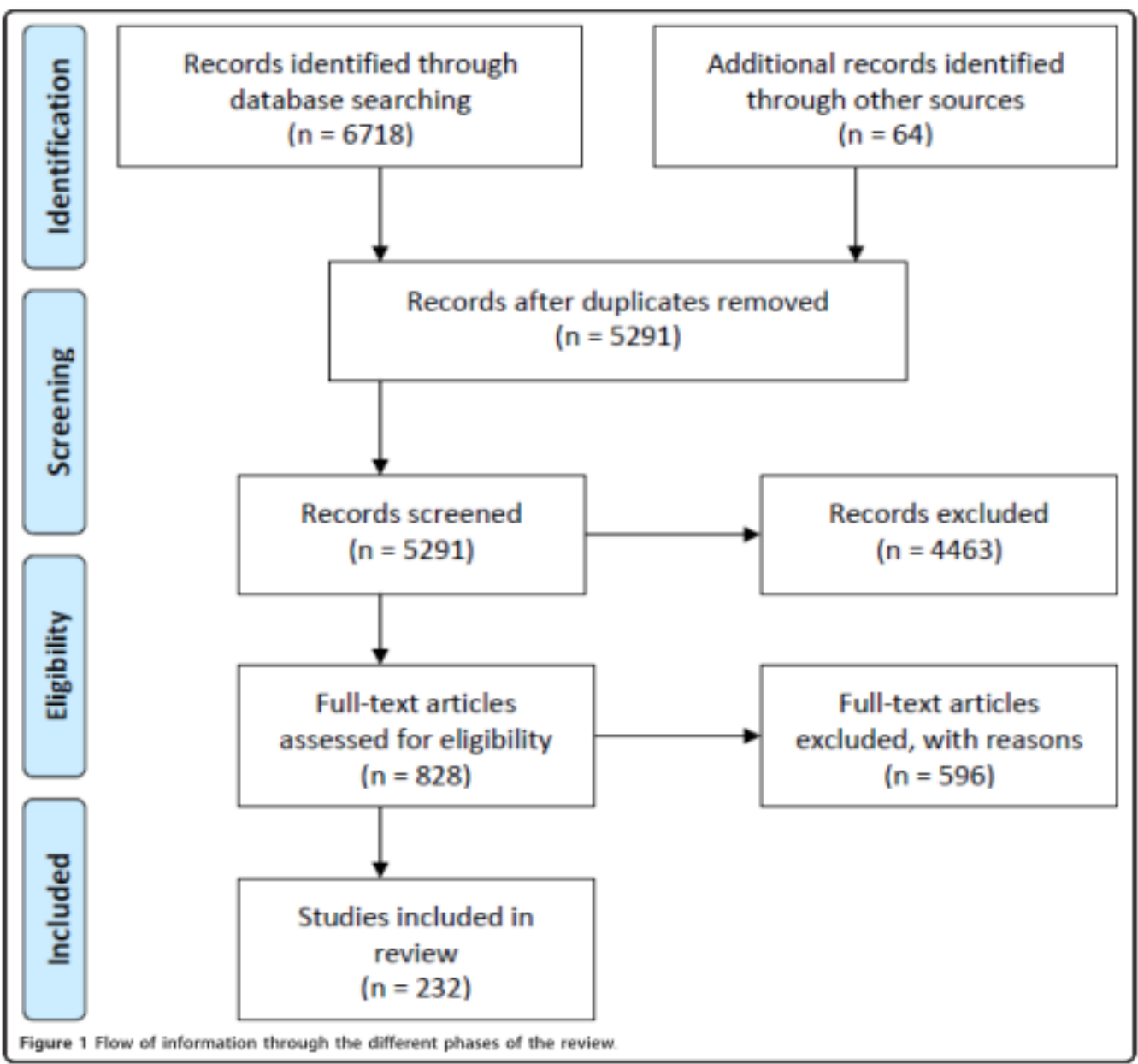

changes in body weight, BMI, and weight status among children and youth who successfully decreased their sedentary time [51-60]. Three intervention studies [61-63] reported that although sedentary behaviour decreased, there was no change in weight status (measured through BMI and skinfold thickness): however, these studies had relatively short follow-up periods ( $\sim 1$ year) and no control group leading the author: hypothesized that a longer follow up period was needed to detect a significant change in body composition. While nine-teen longitudinal studies reported that children who watched greater amounts of TV at baseline saw steeper increases in BMI, body weight and fat mass over time [64-82], nine longitudinal studies reported no significant relationship between time spent sedentary and weight status or fat mass [61-63,83-89]. Of the 119 cross sectional studies, 94 reported that increased sedentary time was associated with one or more of increased fat mass, increased BMI, increased weight status and increased risk for being overweight [28,90-182]. Risk for obesity increased in a dose response manner with increased time spent engaging in sedentary behaviours $(92,106,110,128,156,178]$. Twenty-five cross sectional studies reported no significant relationship between 
Table 2 Summary of characteristics of included studies

\begin{tabular}{|c|c|c|c|c|c|c|c|c|c|c|c|c|}
\hline \multirow{2}{*}{ First Author } & \multirow[b]{2}{*}{ Year } & \multirow{3}{*}{ Country } & \multirow{3}{*}{ Grade } & \multirow{3}{*}{$\begin{array}{l}\text { Age } \\
\text { Range }\end{array}$} & \multirow{3}{*}{ Mean age } & \multicolumn{3}{|c|}{$n$ analyzed } & \multirow{2}{*}{\multicolumn{2}{|c|}{$\begin{array}{l}\text { Units of } \\
\text { sedentary } \\
\text { behaviour }\end{array}$}} & \multirow{3}{*}{ Exposure } & \multirow[b]{2}{*}{ Outcome } \\
\hline & & & & & & Total & Boys & Girls & & & & \\
\hline \multicolumn{8}{|c|}{$\begin{array}{l}\text { RANDOWIZED CONTROLLED } \\
\text { THLALS }\end{array}$} & & & & & \\
\hline Epstein LH [265] & 1995 & US & & $8-12$ & 10.1 & 61 & & & hour & week & TV & $B C$ \\
\hline Epstein LH $[50]$ & 2008 & US & & $4-7$ & 6 & 20 & 37 & 33 & hour & dyy & TV & $\mathrm{BC}$ \\
\hline Goldfield GS [264] & 2006 & Canbada & & $8-12$ & 10.4 & 30 & 13 & 17 & $\min$ & day & TV & $B C$ \\
\hline Gortmaker SL [5]] & 1995 & US & & & 11.7 & 1295 & 668 & 627 & hour & $d z y$ & TV & $B C$ \\
\hline Hughes AR [262] & 1991 & Scotland & & $5-11$ & 8.8 & 134 & 59 & 74 & hour & $d y y$ & $\$ B$ & $B C$ \\
\hline Robinson TN [58] & 1999 & US & & & & 192 & & & hour & week. & TV, GMMES & $B C$ \\
\hline Robinson TN [221] & 2003 & US & & $8-10$ & 9.5 & 61 & 0 & 61 & hour & week. & TV & BC, SE \\
\hline Shelson D [263] & 2007 & Austerdia & & $3-10$ & 7.5 & 43 & 20 & 23 & hour & day & TV & $B C$ \\
\hline \multicolumn{13}{|c|}{ INTERVENTION STUDES } \\
\hline Epstein tH $|56|$ & 2000 & US & & $8-12$ & 105 & 76 & 24 & 52 & hour & menth & SB. ST & $\mathrm{BC}, \mathrm{FIT}$ \\
\hline Epstein LH [SS] & 2004 & uS & & $8-12$ & 9.8 & 60 & 23 & 39 & times & week & TV & $\mathrm{AC}$ \\
\hline Epstein LH $|60|$ & 2005 & US & & $8-16$ & & 58 & 28 & 30 & hour & dsy & SB, TV & $B C$ \\
\hline Gentile DA [61] & 2009 & US & & & 9.6 & 1323 & 685 & 674 & hour & $d \approx y$ & ST & $B C$ \\
\hline Goldfield G5 [52] & 2007 & Canada & & $8-12$ & 104 & 30 & 13 & 17 & hour & $d z y$ & 58 & BC, SE \\
\hline Hamison M [62] & 2003 & reland & & & 102 & 312 & 177 & 135 & $\min$ & $d y y$ & TV, ST & $\mathrm{BC}$ \\
\hline Ochoa MC [S3] & 2007 & Spain & & $6-18$ & 11.6 & 370 & 196 & 174 & hour & week & TV & $B C$ \\
\hline Salmon J [51] & 2008 & Australis & & 1011 & 108 & 311 & 152 & 159 & hour & dsy & TV & $B C$ \\
\hline $\sin 0 n C[54]$ & 2002 & France & & & 11.7 & 954 & 468 & 486 & hour & $d z y$ & TV, COMP & $B C, S E$ \\
\hline Tanasescu M [55] & 2000 & Puerto Hico & & $7-10$ & 9.2 & 53 & 22 & 31 & hour & $d z y$ & TV & $B C$ \\
\hline LCNGITUCINAL STUOES & & & & & & & & & & hour & & \\
\hline Aires L [83] & 2010 & Portugal & & $11-19$ & & 345 & 147 & 198 & hour & dsy & SCAEEN & $\mathrm{BC}, \mathrm{FI}$ \\
\hline Berkey CS [76] & 2003 & US & & $10-15$ & & 11887 & 5120 & 6767 & hour & dyy & TV, GMMES & $B C$ \\
\hline Bhargava A [77] & 2006 & US & & & & 7635 & & & $\min$ & $d z y$ & TV & $B C$ \\
\hline Blair NJ [68] & 2007 & England & & & 5.5 & 591 & 287 & 304 & hour & $d y$ & SB, TV & $B C$ \\
\hline Bornadsile KE [BG] & 2008 & US & & & 11.2 & 1092 & 501 & 591 & hour & woek & TV & $B C$ \\
\hline Burke V [71] & 2006 & Australis & & & $7.6 / 10.8$ & 1569 & 690 & 648 & hour & week & SCAEEN & $B C$ \\
\hline Chen IL [78] & 2007 & Chinese & & $7-8$ & 7.52 & 307 & 147 & 160 & hour & dyy & TV, GMMES & $B C$ \\
\hline Danner PW [66] & 2006 & Us & & & & 7334 & 3674 & 3660 & hour & $d z y$ & TV & $B C$ \\
\hline Dasgupea K [215] & 2006 & Canada & & & $\begin{array}{l}12.7 / 15.1 / \\
170\end{array}$ & 662 & 319 & 343 & hour & week & SB, TV & MS \\
\hline Dry AS [BS] & 2009 & US & & $8-14$ & & 556 & 237 & 279 & $\min$ & dsy & TV & $B C$ \\
\hline Dietz WH [181] & 1985 & US & & $12-17$ & & 2153 & & & hour & $\mathrm{d} y \mathrm{y}$ & TV & $B C$ \\
\hline Elgar FJ [D9] & 2005 & Wales & & & 11.7 & 654 & 293 & 361 & hour & week & TV & $B C$ \\
\hline Elgar FJ [79] & 2005 & Wales & & & 15.3 & 392 & 181 & 211 & hour & week & TV & $B C$ \\
\hline Ennemoser M [237] & 2007 & German & & 68 & & 332 & & & $\min$ & $\mathrm{d} w y$ & TV & $S E, A A$ \\
\hline Fulton JE [94] & 2009 & US & & $10-18$ & & 472 & 245 & 227 & $\min$ & $d s y$ & TV & $B C$ \\
\hline Gable 5 [70] & 2007 & US & & & & 8000 & & & hour & day & TV & $B C$ \\
\hline Hancox RJ [B8] & 2004 & New Zealand & & $5-15$ & & 1013 & & & hour & $d \approx y$ & TV & $B C, M S$ \\
\hline Hancox RI $\mid 72\rceil$ & 2006 & New Zealand & & $5-15$ & & 603 & 372 & 339 & hour & $d y y$ & SCHEEN & $\mathrm{AC}$ \\
\hline Henderson VR $\mid k T]$ & 2007 & US & & $11-19$ & & 2379 & 0 & 2379 & hour & $\mathrm{d} z y$ & TV, SCREEN & $B C$ \\
\hline Hesketh K (80) & 1997 & Australis & & $5-10$ & 7.6 & 1278 & 630 & 648 & hour & dsy & SCREEN & $B C$ \\
\hline Hesketh K [8O] & 1997 & Austedis & & $8-13$ & 10.7 & 1278 & 630 & 648 & hour & dyy & SCREEN & $B C$ \\
\hline Hesketh K. [64] & 2009 & Austoralia & & $5-10$ & 7.7 & 1943 & 972 & 971 & hour & $d z y$ & TV, GMMES & $B C$ \\
\hline Hesketh K $\mid 64]$ & 2009 & Australia & & $8-13$ & & 1569 & 816 & 753 & hour & $d y y$ & TV, GAMES & $B C$ \\
\hline Jackson LA [223] & 2009 & US & & & 12 & 500 & 235 & 26,5 & hour & dsy & $\begin{array}{l}\text { COWP, } \\
\text { SCREEP }\end{array}$ & $\Phi$ \\
\hline Jago R [82] & 2005 & US & & $5-6$ & 6.5 & 138 & 65 & 73 & $\min$ & $\mathrm{ht}$ & SB, TV & $B C$ \\
\hline $\operatorname{Jan} 2 \mathrm{KF}[73]$ & 2005 & us & & & $5.6 / 8.6$ & 378 & 176 & 202 & hour & dæy & SCAEEN & $B C$ \\
\hline Johrson JG [41] & 2007 & US & & & & & & & hour & $d y$ & TV & $A A$ \\
\hline
\end{tabular}


Table 2 Summary of characteristics of included studies (Continued)

\begin{tabular}{|c|c|c|c|c|c|c|c|c|c|c|c|c|}
\hline Kaur H [75] & 2003 & US & & $12-17$ & & 2223 & 1149 & 1074 & hour & dyy & TV & $B C$ \\
\hline Lajunen HA [128] & 2007 & Finland & & $15-19$ & & 5184 & & & hour & & $5 B$ & $B C$ \\
\hline Lonner W [238] & 1985 & US & & 9.19 & 14.2 & 367 & & & hour & $d y$ & TV & AA \\
\hline Maffecs C [99] & 1998 & raly & & & 87 & 298 & 148 & 150 & $\min$ & dsy & SCAEEN & $A C$ \\
\hline Mistry K [229] & 2007 & US & & & & & & & hour & dey & TV & PAO \\
\hline Mitchel JA [49] & 2009 & UK & & $11-12$ & 118 & 5434 & 2590 & $2 B 44$ & hour & $d z y$ & 58 & $B C, F I T$ \\
\hline Must A [87] & 2007 & US & & $10-17$ & & 156 & 0 & 156 & hour & $d z y$ & SB, SCFEEN & $B C$ \\
\hline OBtien $M[f r]$ & 2007 & US & & $2: 12$ & & 653 & & & hour & woek & TV & $B C$ \\
\hline Parscrs TJ [74] & 2005 & England/Scr & dand/Wa & & $11 / 16$ & 17733 & & & hour & dsy & TV & $\mathrm{BC}$ \\
\hline Purslow LR [63] & 2008 & England & & $8-9$ & & 345 & 176 & 169 & $\min$ & $\mathrm{d} s y$ & $S B$ & $\mathrm{BC}$ \\
\hline Timperio A [55] & 2008 & Austealia & & $10-12$ & & 344 & 152 & 192 & times & week & SB, SCFEEN & $B C$ \\
\hline Treuth MS [29) & 2007 & US & & & 119 & 964 & 0 & 984 & $\min$ & $d z y$ & $S B$ & $B C$ \\
\hline Treuth MS [27] & 2009 & US & & & 13.9 & 984 & 0 & 984 & $\min$ & dsy & $\$ B$ & $\theta C$ \\
\hline Wasje.KS [206] & 2009 & US & & $6.35-7.25$ & & 214 & & & hour & dsy & SCREEN & AT \\
\hline \multicolumn{13}{|c|}{ CAOSS SECTONAL STUOES } \\
\hline Al SH [192] & 2009 & memationa & & $12-18$ & & 17715 & 8503 & 9212 & hour & dzy & TV & $B C$ \\
\hline Albarwani 5 [207] & 2009 & Oman & & $15-16$ & & 529 & 245 & 284 & hour & week & TV, COMP & FIT \\
\hline Ahes JG [191] & 2009 & Brasil & & $7+10$ & & 733 & 407 & 326 & hour & $\mathrm{d} w y$ & TV & $\mathrm{AC}$ \\
\hline Aman J [218] & 2009 & Swreden & & $11-18$ & 14.5 & 2093 & 1016 & 991 & hour & week & TV, COMP & MS \\
\hline Andersen LF [155] & 2005 & Nanway & & $8-14$ & & 1432 & 702 & 730 & hour & dsy & TV & $\mathrm{BC}$ \\
\hline Andersen AE [142] & 1998 & US & & $8-16$ & & 4063 & 1985 & 2071 & hour & $d y$ & TV & $B C$ \\
\hline Anderson SE [103] & 2008 & US & & $4-12$ & 8 & 2964 & 1509 & 1455 & hour & $d \approx y$ & TV & $B C$ \\
\hline Ammstrong CA [213] & 1998 & US & & & 9.28 & 588 & 304 & 284 & hour & dsy & TV & FI \\
\hline Assrete Ph [183] & 2009 & US & & $3-13$ & 8.5 & 324 & 182 & 142 & hour & dsy & SCREEN & $\mathrm{BC}$ \\
\hline Aucote HM [163] & 2009 & Austedis & $5-6$ & & 11.09 & 393 & 198 & 195 & hour & week & TV, GMMES & $B C$ \\
\hline Barlow SE [151] & 2007 & US & & $6-17$ & 12.1 & 52845 & & & hour & dæy & TV & $B C$ \\
\hline Basaldua N [109] & 2008 & Mexice & & $6-12$ & 89 & 551 & 278 & 273 & hour & $d y$ & TV & $B C$ \\
\hline Beliske F [123] & 2007 & France & & $9-11$ & & 1000 & 500 & 500 & hour & dsy & TV & $\theta C$ \\
\hline Berhey CS [90] & 2000 & US & & & Sep-14 & 10769 & 4620 & 6149 & hour & dsy & TV & $\mathrm{BC}$ \\
\hline Beyerlein A [105] & 2008 & Germary & & $45-73$ & & 4967 & 2585 & 2382 & hour & day & TV & $B C$ \\
\hline Boone JE [164] & 2007 & US & & & 15.9 & 9155 & 4879 & 4276 & hour & week & SCAEEN & $B C$ \\
\hline Boone-Heinonen J [104] & 2008 & US & & $11-21$ & & 9251 & & & hour & & SB & $B C$ \\
\hline Boutele KN [130] & 2007 & US & & $16-18$ & & 1726 & 890 & 836 & hour & dyy & TV & $\mathrm{AC}$ \\
\hline Brodersen MH [235] & 2005 & England & & & 11.8 & 4320 & 2578 & 1742 & hour & week & SB & $\Phi$, PAO \\
\hline $\begin{array}{l}\text { Bukara-fodujkovic G } \\
\text { [96] }\end{array}$ & 2009 & Bosnia & & $11-12$ & 11.5 & 1204 & 578 & 626 & hour & dyy & TV, COMP & $B C$ \\
\hline Burte NF (119) & 2007 & US & & $6-17$ & 108 & 897 & 441 & 456 & hour & $d y$ & SCAEEN & $\mathrm{BC}$ \\
\hline Caldas S [245] & 1999 & US & & $4-19$ & & 34542 & & & hour & dsy & TV & $\mathrm{Ma}$ \\
\hline Carvalhal MM [131] & 2007 & Portugal & $10-11$ & & & 3365 & 1755 & 1610 & hour & dsy & TV, COMP & $\mathrm{BC}$ \\
\hline Chaput J [154] & 2006 & Cansda & & $5-10$ & 66 & 422 & 211 & 211 & hour & dyy & SCREEN & $B C$ \\
\hline Chen Mr [78] & 2007 & Taiwan & & $13-18$ & $15: 03$ & 660 & 351 & 309 & hour & $d z y$ & TV, COMP & $\begin{array}{l}B C, S E, \\
\text { PHO }\end{array}$ \\
\hline Chowhan I [232] & 2007 & Cansda & & $12-15$ & & 2666 & & & hour & dsy & TV & PAO \\
\hline Christcforids A [95] & 2009 & Greece & & $4-18$ & 11.41 & 1549 & 735 & 814 & hour & dsy & SCREEN & $\mathrm{BC}, \mathrm{FIT}$ \\
\hline Colins AE [149] & 2008 & indonesid & & $12-15$ & & 1758 & 815 & 916 & hour & dsy & TV, COMP & $B C$ \\
\hline Colwell J [200] & 2003 & lapan & & $12-13$ & & 305 & 159 & 146 & hour & $d y y$ & SCAEEN & $B C$, PFO \\
\hline Cocper H [247] & 1999 & US & $7-11$ & & & 424 & 225 & 199 & hour & $d z y$ & TV & AA \\
\hline Crespo $\subset[177]$ & 2001 & US & & $8-16$ & & 4069 & 1994 & 2075 & hour & dsy & TV & $\mathrm{BC}$ \\
\hline $\mathrm{Da} C \mathrm{CA}[157]$ & 2003 & Araol & & $7-10$ & & 446 & 107 & 107 & hour & dsy & TV & $\mathrm{BC}$ \\
\hline Dasgupea K. [215] & 2007 & Cansda & & $13-17$ & & 1267 & & & hour & week & SCAEEN & MS \\
\hline Detva J [125] & 2007 & Us & & & & 11265 & 5274 & 5991 & hour & week & TV & $B C$ \\
\hline Dietz MH [181] & 1965 & US & & $12-17$ & & 6671 & & & hour & $d x y$ & TV & AA \\
\hline Dietz WH [181] & 1985 & US & & $6-11$ & & 6065 & & & hour & dsy & TV & $\mathrm{BC}, \mathrm{MA}$ \\
\hline Dollman J [211] & 2006 & Austealis & 6 & $10-11$ & & 843 & 439 & 404 & min & Day & TV & AT \\
\hline
\end{tabular}


Table 2 Summary of characteristics of included studies (Continued)

\begin{tabular}{|c|c|c|c|c|c|c|c|c|c|c|c|c|}
\hline Dumais SA [255] & 2009 & US & & $10-12$ & & 15850 & & & hour & & TV & AA \\
\hline Dominick $R$ [225] & 1964 & US & 10.11 & $14-18$ & & 250 & 110 & 140 & hour & Day & TV, GAME & SE, PFO \\
\hline Ekermarn $K C$ [175] & 2002 & US & & $14: 18$ & & 15143 & & & hour & $d y y$ & TV & $\mathrm{BC}$ \\
\hline Elsermarn JC [113] & 2008 & US' & & & 16.2 & 12464 & 6080 & 6394 & hour & dsy & TV & $A C$ \\
\hline Ekelund U [130] & 2006 & Europe & & $9-16$ & & 1921 & 911 & 1010 & hour & dsy & TV & $\mathrm{BC}, \mathrm{MS}$ \\
\hline Fetler $M[249]$ & 1964 & US & 6 & & & 10603 & & & hour & day & SCAEEN & AA \\
\hline Forshee RA [201] & 2004 & US & & $12-16$ & 14 & 2216 & 1075 & 1141 & hour & $d y y$ & TV & $\mathrm{BC}$ \\
\hline Forshee RA [188] & 2009 & us & & $5 \cdot 18$ & & 1459 & 734 & 725 & hour & week & SCAEEN & $A C$ \\
\hline Gaddy GD [257] & 1986 & US & & & & 5074 & & & hour & dsy & TV & $M$ \\
\hline Gismmattei J][140] & 2003 & US & & $11-14$ & 126 & 385 & 186 & 199 & hour & dey & TV & $B C$ \\
\hline Gibson 5 [156] & 2004 & England & & $7-18$ & & 1294 & 655 & 639 & $\min$ & $d z y$ & TV & $B C$ \\
\hline Gomez UF [150] & 2007 & Colombia & & $5-12$ & & 11137 & 5539 & 5598 & hour & $d y y$ & TV, GMMES & $B C$ \\
\hline Gorden-Larsen P [176] & 2002 & US & & $11-19$ & 15.9 & 12759 & 6290 & 6496 & hour & week & TV, GMMES & $B C$ \\
\hline Gortmaker SL. [143] & 1996 & US & & $10-15$ & 11.5 & 746 & 389 & 358 & hour & $\mathrm{d} s y$ & TV & $\mathrm{BC}$ \\
\hline Gortmaker SL [5]] & 1999 & US & & $6-11$ & & 1745 & & & $\min$ & week & TV & $S E, A A$ \\
\hline Gormaker SL [5]] & 1999 & US & & $12-17$ & & 1745 & & & $\min$ & week & TV & $\mathrm{SE}, \mathrm{AA}$ \\
\hline Graf C $[167]$ & 2004 & Germary & & & 6.8 & 344 & 177 & 167 & hour & $d z y$ & TV, COMP & $\mathrm{BC}$ \\
\hline Guxser SM [40] & 2005 & Germary & 6 & & 11.83 & 323 & 175 & 148 & hour & $d x y$ & TV & $\mathrm{MA}$ \\
\hline Hardy LL [133] & 2006 & Australis & & $11-15$ & & 2750 & 1446 & 1304 & hour & $d s y$ & SCAEEN & AT \\
\hline Hemandez B [178] & 1999 & Mexico & & $9-16$ & & 461 & 244 & 217 & hour & dyy & TV & $B C$ \\
\hline Hirschler V [144] & 2009 & Argentinà & & $7-11$ & 89 & 330 & 168 & 162 & hour & $d z y$ & TV & $B C$ \\
\hline Holder MD |222] & 2009 & Canada & & $8-12$ & & 375 & 252 & 262 & hour & $d z y$ & SCAEEN & $\mathbb{E}$ \\
\hline Hume C [190] & 2009 & Nectherlands & & & 13 & 580 & 237 & 303 & hour & dsy & SCAEEN & $A C$ \\
\hline Islam-Zwart K [195] & 2008 & US & & & & 480 & 198 & 282 & hour & dsy & TV & $\mathrm{BC}$ \\
\hline Jacksen LA [223] & 2009 & US & & & 12.18 & 515 & 259 & 256 & hour & day & $\begin{array}{l}\text { GAMES, } \\
\text { COWP }\end{array}$ & $A A$ \\
\hline Janssen I [166] & 2004 & Canada & & $11-16$ & & 5890 & 2812 & 3078 & hour & $d z y$ & TV, COMP & $B C$ \\
\hline $\operatorname{lanz} \mathrm{K}[174]$ & 2002 & US & & 46 & 5.3 & 462 & 216 & 246 & hour & dsy & TV & $\mathrm{BC}$ \\
\hline Jaruratanssirkul S [241] & 2009 & Thaland & $7-12$ & & 15.9 & 1492 & 562 & 929 & hour & & GAMES & AA \\
\hline Jotrison CC [41] & 2007 & US & & & 12 & 1397 & 0 & 1397 & hour & $d y y$ & SB & $S E$ \\
\hline Katzmarzyk PT [197] & 1996 & Canada & & 9.18 & & 784 & 423 & 361 & $\min$ & $d x y$ & TV & $\mathrm{BC}, \mathrm{FIT}$ \\
\hline Katzmarzyk PT [184] & 1996 & Canada & & & & 640 & 356 & 284 & hour & $d z y$ & TV & $\mathrm{BC}, \mathrm{FIT}$ \\
\hline Kautisinen S [135] & 2005 & Finland & & $14-18$ & & 6515 & 2916 & 3599 & hour & dzy & SCAEEN & $\mathrm{AC}$ \\
\hline Keith TZ [256] & 1986 & US & high sct & hool seriors & & 28051 & & & hour & dsy & TV & $A A$ \\
\hline Kein-Platat C [165] & 2005 & France & & & 12 & 2714 & 1357 & 1357 & hour & week & SB & $B C$ \\
\hline Kosti Al [196] & 2007 & Greece & & $12-17$ & & 2006 & 1021 & 987 & hour & dzy & TV & $B C$ \\
\hline Kristjarsson AL [243] & 2009 & Iceland & & $14-15$ & & 5810 & 2807 & 3004 & hour & $d x y$ & TV & AA \\
\hline Kunssche E [230] & 2006 & Imemational & & $11-15$ & & 31177 & & & hour & $d y y$ & TV & $\mathrm{PAO}$ \\
\hline Kuriyan $\mathrm{A}[117]$ & 2007 & India & & $6-16$ & & 598 & 324 & 274 & hour & $d s y$ & TV & $\mathrm{BC}$ \\
\hline Lagiou A [ [1600] & 2008 & Greece & & $10-12$ & & 633 & 316 & 317 & hour & dsy & TV, GAMES & $B C$ \\
\hline Lajcus M [92] & 2009 & Mexico & & $11-18$ & 139 & 9132 & 3519 & 5613 & hour & $\mathrm{d} z y$ & TV & $B C$ \\
\hline Lajunen HR [128] & 2007 & Finland & & & 176 & 4098 & 1981 & 2117 & hour & week & comp & $B C$ \\
\hline Lasserre AM [1 16] & 2007 & Switzerland & & $10.1-149$ & 12.3 & 5207 & 2621 & 2586 & hour & dsy & TV & $B C$ \\
\hline Lsurson KR [107] & 2008 & US & & $7-12$ & & 709 & 318 & 391 & hour & week & SCAEEN & $B C$ \\
\hline Lacarou C [217] & 2009 & Cyporus & & & 11.7 & 622 & 306 & 316 & hour & day & TV & MS \\
\hline Leatherdale ST [11] & 2006 & Canada & & $14-19$ & & 25416 & 12806 & 12610 & hour & $d y y$ & TV & BC, PFO \\
\hline Lioret \$ [127] & 2007 & France & & $3-14$ & & 1016 & 528 & 488 & hour & $d y$ & SB, TV, COMP & $\mathrm{BC}$ \\
\hline Lobela F [20e] & 2009 & US & & $14-18$ & & $\$ 210$ & 0 & $\$ 210$ & hour & $d x y$ & SCAEEN & AT \\
\hline Lowry A [173] & 2002 & US & & & & 15349 & 7445 & 7828 & hour & dsy & TV & $B C$ \\
\hline Lutfiyya MN [118] & 2007 & US & & $5-17$ & & 7972 & & & hour & d2y & TV & $B C$ \\
\hline Maffeis C [114] & 2008 & traly & & $8-10$ & 9.3 & 1837 & 924 & 913 & hour & $d z y$ & TV & $\mathrm{BC}$ \\
\hline Mark AE [270] & 2008 & US & & $12=19$ & 15.9 & 1803 & 1005 & $7 \times 8$ & hour & $d x y$ & TV & $A C, M S$ \\
\hline McMurray AG [187] & 2000 & US & & $10-16$ & 12.7 & 2389 & 1149 & 1240 & hour & dsy & TV & $\theta C$ \\
\hline Whas C [193] & 2009 & Greece & & $12-17$ & 14.4 & 2008 & 1021 & 997 & hour & $d s y$ & SCREEN & $B C$ \\
\hline
\end{tabular}


Tremblay et af. intematianal Jounal of Behawioral Mutrition and Physical Activity 2011,

Page 9 of 22 hittp//wawijibnpa.org/content/3/1/98

Table 2 Summary of characteristics of included studies (Continued)

\begin{tabular}{|c|c|c|c|c|c|c|c|c|c|c|c|c|}
\hline Mikolajocyk RT [194] & 2008 & Germarty & & $11-17$ & 13.5 & 4878 & 2433 & 2445 & hour & $\begin{array}{l}\text { low' } \\
\text { hight }\end{array}$ & $5 B$ & $B C$ \\
\hline Moraes SA [135] & 2006 & Mexico & & $6-14$ & $80 / 113$ & 662 & 343 & 339 & hour & week & & \\
\hline Morgenstem M [94] & 2009 & Germanyuls & & $10-17$ & 128 & 4810 & 2294 & 2516 & hour & dsy & SCAEEN & $\mathrm{AC}$ \\
\hline Morgenstem M [94] & 2009 & GermarnyUS & & $12-16$ & 14 & 4473 & 2239 & 2224 & hour & dsy & SCREEN & $B C$ \\
\hline Mota J [199] & 2006 & Portugal & & & 14.6 & 450 & 220 & 230 & hour & day & TV, COMP & $B C$ \\
\hline Mules w [179] & 1999 & Germary & & $5-7$ & & 1468 & 739 & 729 & hour & $d z y$ & TV & $B C$ \\
\hline Nagel G (193] & 2009 & Germary & & 6.9 & 7,57 & 1079 & & $4 \times 8$ & hour & $\mathrm{d} w y$ & TV, GAMES & $B C$ \\
\hline $\begin{array}{l}\text { nastassea-Vlachou K } \\
{[240]}\end{array}$ & 1996 & Greece & & $6-13$ & & 4090 & 2279 & 2411 & hour & $d s y$ & TV & MA \\
\hline Nawal LM [148] & 1996 & US & & $5-18$ & & 62976 & & & hour & day & TV, COMP & $B C$ \\
\hline Netson MC [233] & 2006 & US & & $7-12$ & & 11957 & 5979 & 5978 & hour & $d a y$ & SCAEEN & PAO \\
\hline $\begin{array}{l}\text { Neumark-Sztainer D } \\
\text { [224] }\end{array}$ & 2004 & US & & $11-18$ & 149 & 4746 & 2382 & 2354 & hour & week & TV & SE, PHO \\
\hline Nogueira th [45] & 2009 & Brasl & & $83-16.8$ & 13 & 326 & 204 & 122 & hour & dsy & $S B$ & $B C$ \\
\hline Cosrzanek E [180] & 1994 & US & & $9-10$ & 10.1 & 2379 & 0 & 2379 & hour & week & TV & $B C$ \\
\hline Oharnessian om [226] & 2009 & Us & & $14-16$ & 14.99 & 328 & 138 & 190 & hour & $d z y$ & SCAEEN & $\begin{array}{l}\mathrm{SE}, \mathrm{PHO}, \\
\mathrm{AA}\end{array}$ \\
\hline Ortega FB [122] & 2007 & Spain & & $13 \cdot 18.5$ & 15.4 & 2859 & 1357 & 1502 & hour & $d x y$ & $\$ B$ & $\theta C$ \\
\hline Overby NC [219] & 2009 & Narway & & $6-19$ & & 723 & 375 & 348 & $\min$ & $d s y$ & TV & \\
\hline Oemert E [42] & 2002 & Turkey & & & & 689 & 343 & 306 & hour & doy & TV & PAO, AA \\
\hline Padez C 1991 & 2009 & Portugal & & $7-9$ & & 3390 & 1696 & 1694 & hour & $d y y$ & TV & $B C$ \\
\hline Page FM [234] & 2001 & Phlippine & & & 15,1 & 3307 & 1267 & 1819 & hour & week & TV & PAO \\
\hline Pate FR [210] & 2006 & US & & $12-19$ & 15.4 & 3287 & 1685 & 1601 & hour & dsy & TV & FT \\
\hline Patrick K [169] & 2004 & US & & $11-15$ & 12.7 & 878 & 407 & 471 & $\min$ & dsy & TV & $B C$ \\
\hline Pratt C [101] & 2008 & US & & & 12 & 1458 & 223 & 1235 & hour & dsy & 58 & $B C$ \\
\hline Purath J [185] & 1995 & US & $3-5$ & & & 365 & 189 & 176 & hour & $\mathrm{d} 3 y$ & TV & $B C, M S$ \\
\hline Ramos E [126] & 2007 & Portugal & & 13 & & 2161 & 1045 & 1116 & $\min$ & week & SR, TV, COMP & $B C$ \\
\hline Rapp K [138] & 2005 & Germary & & & 6.2 & 2140 & 1015 & 1125 & hour & $\mathrm{d} z y$ & TV & $\theta C$ \\
\hline Pidley-Jchreon R [252] & 1983 & US & $5-8$ & & & 290 & & & hour & dsy & TV & $A A$ \\
\hline Proberts of [250] & 1984 & US & & & & 539 & & & hour & week & TV & AA \\
\hline Ropbinson TN [58] & 1999 & us & & & 12.4 & 971 & 0 & 971 & hour & dyy & TV & $B C$ \\
\hline $\begin{array}{l}\text { Ruangdaraganon } \mathrm{N} \\
\text { [141] }\end{array}$ & 2002 & Thalland & & $6-12$ & 9.4 & 4197 & 2126 & 2035 & hour & $d y y$ & TV & $B C$ \\
\hline Russ Sh [147] & 2009 & uS & & $6-17$ & & 54863 & 28153 & 26710 & hour & dsy & SCREEN & BC, SE \\
\hline Sokamoto A [236] & 1994 & Japan & $4-6$ & & & 307 & 165 & 142 & times & week & GAMES & PAO \\
\hline Sakamoto A [236] & 1994 & lapan & $4 \cdot 6$ & & & 537 & 287 & 250 & hour & week & $\begin{array}{l}\text { COWP, } \\
\text { GANES }\end{array}$ & PAO \\
\hline Sakamoto A [235] & 1994 & tapan & 4.5 & & & 118 & 118 & 0 & hour & week & $\begin{array}{l}\text { COMP, } \\
\text { GANES }\end{array}$ & PAO \\
\hline Salmon J [136] & 2006 & Austedis & & $5-12$ & & 1560 & 743 & 817 & hour & dey & TV & $\mathrm{BC}$ \\
\hline Sädinhà L8 [48] & 2008 & Portugal & & $9-10$ & 98 & 306 & 161 & 147 & hour & dyy & SB & M5 \\
\hline Scont LF [254] & 1958 & U5 & $6-7$ & & & 407 & & & hour & & TV & AA \\
\hline Sharf I [244] & 2006 & US & & $10-14$ & & 6922 & 3169 & 3353 & hour & $d x y$ & TV, GAMES & $\mathrm{PAO}, \mathrm{AA}$ \\
\hline Sharf I [260] & 2010 & us & & $9-15$ & 12 & 4508 & 2209 & 2299 & hour & $d s y$ & TV, GAMES & $\mathrm{AA}$ \\
\hline Shejwal B [246] & 2006 & india & & & 16.05 & 654 & 368 & 296 & hour & day & TV & $A A$ \\
\hline Shields M [162] & 2006 & uscan & & $2-17$ & & 8661 & & & hour & d $3 y$ & SB, TV & $B C$ \\
\hline Shin N [239] & 2004 & Us & & $6-13$ & 9 & 1203 & 605 & 598 & $\min$ & dzy & TV & AA \\
\hline Singh GK [105] & 2003 & US & & $10-17$ & & 46707 & 24072 & 22635 & hour & $d y y$ & TV & $\theta C$ \\
\hline Singh GX [106] & 2003 & US & & $10-17$ & & 46307 & 24072 & 220635 & hour & dsy & TV & $B C$ \\
\hline Skoric MM [258] & 2009 & Singapore & & $8-12$ & 10 & 333 & 180 & 153 & hour & & TV, GMMES & AA \\
\hline Smith BJ [161] & 2007 & $\mathrm{Fij}$ & & $11-16$ & & 443 & 200 & 245 & hour & d $3 y$ & TV & $B C$ \\
\hline Spinks AB [124] & 2007 & Australia & & $5-12$ & & 518 & 282 & 236 & $\min$ & week & SB, SCFEEN & $B C$ \\
\hline Steffen LM [Q8] & 2009 & us & & $8-11$ & & 526 & 256 & 270 & hour & dsy & TV & $A C$ \\
\hline Stettler N [160] & 2004 & Switzerland & & & 8 & 872 & 410 & 462 & hour & dsy & TV, GAMES & $B C$ \\
\hline
\end{tabular}


Table 2 Summary of characteristics of included studies (Continued)

\begin{tabular}{|c|c|c|c|c|c|c|c|c|c|c|c|c|}
\hline Sugiyama $\mathrm{T}[47]$ & 2007 & US & & $12-19$ & 15.9 & 4508 & 2295 & 2213 & hour & $\mathrm{d} z y$ & $5 B$ & MS \\
\hline $\operatorname{Sun} Y[91]$ & 2009 & lapan & & $12-13$ & & 5753 & 2842 & 2911 & hour & $d x y$ & TV & $B C$ \\
\hline Taylar Wc [158] & 2002 & us & & $6-15$ & 11,1 & 509 & 231 & 278 & kal & $d x y$ & $S B$ & $B C$ \\
\hline te Veide SJ [129] & 2007 & Imernational & & $9-14$ & 11.4 & 12538 & 0258 & 6282 & hour & dsy & TV, COMP & $\mathrm{aC}$ \\
\hline Thompson AM [189] & 2009 & Cansda & $\begin{array}{l}3,7 \\
11\end{array}$ & & & $17 m 7$ & 795 & 992 & $\min$ & dsy & TV & $B C$ \\
\hline Toschike AM [112] & 2008 & Germary & & $5-6$ & & 4884 & & & hour & $d \approx y$ & TV & $B C$ \\
\hline Toschike AM [121] & 2007 & Germary & & $5 \cdot 6$ & & 5472 & & & hour & $d y$ & TV & $B C$ \\
\hline Trang NHHD [145] & 2009 & Aurtialis & & $11-16$ & & 2060 & 1332 & 1328 & hour & dsy & SCAEEN & $B C$ \\
\hline Trembisy MS [172] & 2003 & Cansdes & & $7-11$ & & 7261 & & & hour & dsy & TV & $B C$ \\
\hline Treuth WS [27] & 2009 & us & & $11-12$ & 11.9 & 1579 & 0 & 1579 & hour & day & 58 & $B C$ \\
\hline Tsai H [153] & 2007 & Tawan & & $11-12$ & & 2218 & 1146 & 1072 & hour & $d \approx y$ & TV & $B C$ \\
\hline Tsai H [145] & 2009 & Tawan & & $11-12$ & & 1329 & 615 & 672 & hour & $d x y$ & SB. TV & $B C$ \\
\hline Tucker LA [212] & 1987 & US & & & 15.7 & 406 & 406 & 0 & hour & dsy & TV & $\begin{array}{l}\text { AT, SE, } \\
\text { PAO }\end{array}$ \\
\hline Tucher LA [206] & 1986 & US & & & 15.7 & 379 & 379 & 0 & hour & dsy & TV & FIT \\
\hline Tudver LA [214] & 1996 & us & & $9-10$ & 9.8 & 262 & 162 & 100 & hour & $d z y$ & TV & Fा \\
\hline Ussher MH [231] & 1007 & England & & $13-16$ & & 2623 & & & hour & $d y y$ & TV & $P A O, A A$ \\
\hline Utter J [171] & 2003 & US & & & 14.9 & 4480 & 2240 & 2240 & hour & dsy & SCAEEN & $\mathrm{BC}$ \\
\hline Utter J [152] & 2007 & New Zesland & & $5-14$ & & 1743 & $\$ 59$ & 794 & hour & dsy & TV, COMP & $B C$ \\
\hline Vader AM [97] & 2009 & us & & & 11,7 & 11594 & 6162 & 5432 & hour & day & TV & $B C$ \\
\hline van Schie EG [261] & 1997 & Nerherlands & & $10-14$ & 115 & 346 & 171 & 175 & hour & $d x y$ & SCREEN & PAO, AA \\
\hline van Zusphen M [159] & 2007 & Australia & & $4-12$ & 8 & 1926 & 939 & 987 & $\min$ & $d x y$ & TV & $\mathrm{BC}$ \\
\hline Vandewater EA [1/0] & 2004 & US & & $1-12$ & 6 & 2831 & 1444 & 1387 & hour & dsy & $S B, S C R E E N$ & $B C$ \\
\hline Vaughan C [199] & 2007 & Australis & & $11-18$ & 14 & 443 & 189 & 254 & hour & dsy & SCAEEN & $\mathrm{BC}$ \\
\hline $\begin{array}{l}\text { Voente-fodriguez G } \\
{[110]}\end{array}$ & 2006 & Spain & & $13-18.5$ & & 1960 & 1012 & 948 & hour & day & TV, GAMES & $B C$ \\
\hline Volane R [137] & 2005 & Mexice & & $6: 14$ & & 8624 & 258 & 4366 & hour & $d y$ & TV & BC \\
\hline Wshe M [186] & 2003 & Australis & & $5-13$ & 9.1 & 2802 & 1445 & 1417 & hour & week & SCAEEN & $B C$ \\
\hline Wolberg HJ [251] & 1984 & US & $2-6$ & & 13 & 2890 & 1445 & 1445 & hour & $\mathrm{d} s y$ & TV & $A A$ \\
\hline Walberg HJ [253] & 1982 & US & & & 17 & 2001 & 1031 & 970 & hour & dyy & TV & $A A$ \\
\hline Waller ce [202] & 2003 & China & & $6-11$ & 9 & 880 & & & hour & week & TV & $B C$ \\
\hline Wang $Y[120]$ & 2007 & US & & & 11.9 & 496 & 218 & 280 & hour & $d y$ & SCAEEN & $B C$ \\
\hline Welch WW [248] & 1986 & Australis & $3-4$ & 9 & 9 & 1960 & & & & & TV & $\mathrm{MA}$ \\
\hline Wells JC [108] & 2000 & Brasol & & $10-12$ & & 4452 & 2193 & 2258 & hour & dsy & TV & $B C, M S$ \\
\hline Whitt-Glover MC [24] & 2009 & Us & & $6-19$ & & 749 & 351 & 398 & $\min$ & day & 58 & $B C$ \\
\hline Wiggins J [227] & 1967 & Us & $4-12$ & & & 483 & 252 & 231 & $\min$ & $d \geqslant y$ & TV & $\mathrm{SE}, \mathrm{AA}$ \\
\hline Wolf AM [203] & 1998 & us & & $11-14$ & & 552 & 0 & 552 & hour & $d x y$ & TV & $B C$ \\
\hline Wang \& $[100]$ & 2009 & Cansda & & & 15.5 & 2060 & 12906 & 12854 & hour & dsy & SB, SCREEN & $B C$ \\
\hline Zabinski MF [132] & 2007 & us & & $11-15$ & & 878 & 425 & 453 & hour & dsy & $S B$ & $B C$ \\
\hline
\end{tabular}

SR. sedentary behavious: TV, televivion viewing COMP, sompute time; CAME, video game playing: SCDEEN composite measure of 2 or more screen activities lie. television vewing compuser time, or video game playingt BC, body composition MS, measures of metabolic syndrome andfor candiovascular disesse leg. insulin resktances, blood pressuev, SE, self essoem, PFo, peosocial behaviour, AA, academic achievement.

sedentary time and weight status $[24,85,137,183-204]$. One study [131] reported an effect in boys but not girls and one showed an effect in girls but not boys [139]. One study showed that among boys, being underweight was associated with more screen time [111]. The level of evidence reporting on the relationship between sedentary behaviour and body composition was of moderate quality and was classified as Level 2 with a mean Downs and Black score of 20.6 (standard deviation: \pm 1.9 ).
Fitness

Fifteen studies assessed the relationship between time spent engaging in sedentary behaviour and fitness (Table 4). Increased time spent being sedentary was associated with decreased scores for overall physical fitness, $\mathrm{VO}_{2}$ max, cardiorespiratory fitness, and musculoskeletal fitness. An intervention reported that targeting decreased sedentary behaviour lead to increases in aerobic fitness [56]. This study ( $\mathrm{n}=13$ boys and 26 girls, mean age $=$ 
Table 3 Summary table of results showing relation between sedentary behaviour and measures of body composition

\begin{tabular}{|c|c|c|c|}
\hline $\begin{array}{l}\text { Type of } \\
\text { Study }\end{array}$ & $\begin{array}{l}\text { Number of } \\
\text { Studies }\end{array}$ & $\begin{array}{l}\text { Number of } \\
\text { participants }\end{array}$ & Narrative recommendation and main findings \\
\hline$\overline{\mathrm{FCT}}$ & 8 & 1896 & Reductions in sedertary behaviaur are directly related to improved body composition. \\
\hline Intervention & 10 & 3547 & $\begin{array}{l}\text { TV wathing and overweightrobesity were related in a dose-resporse manner tie. those who } \\
\text { wasched more TV were more likely to be overweighwobesel }\end{array}$ \\
\hline Longitudinal & 33 & 85753 & $\begin{array}{l}\text { TV watching and overweight/obesity were related in a dose-resporse manner the, those who } \\
\text { wanched mone TV were mare likely to be ovenweight/obescl. }\end{array}$ \\
\hline $\begin{array}{l}\text { Cross } \\
\text { sectional }\end{array}$ & 119 & 691759 & $>2$ hrs of sedernary behwiour nelated to increased risk of being overweight or obese. \\
\hline $\begin{array}{l}\text { Total of al } \\
\text { studies }\end{array}$ & 170 & 782884 & 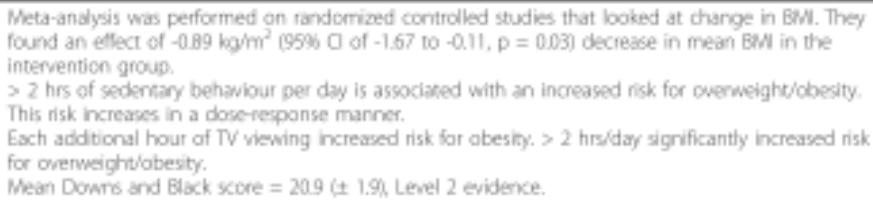 \\
\hline
\end{tabular}

10.5 years) showed that an intervention to decrease targeted sedentary behaviours (watching TV, playing computer games, talking on the telephone, or playing board games) led to increases in both physical activity and nontargeted sedentary behaviours. Longitudinal evidence was conflicting. One longitudinal study showed that $>2$ hours per day of TV and computer use was associated with decreased musculoskeletal fitness [205]; while the second longitudinal study found no association between increased screen time and decreased fitness. Eight of 12 cross sectional studies showed that greater than 2 hours of screen time per day was associated with decreased $\mathrm{VO}_{2} \max$, lower cardiorespiratory fitness, and lower aerobic fitness [95,206-212]. Two studies showed weak relationships between television watching and fitness $[197,213]$. Two studies showed no consistent association between television viewing and aerobic and musculoskeletal fitness $[184,214]$. The level of evidence related to fitness was classified as Level 3 with a mean Downs and Black score of 20.9 (standard deviation: \pm 2.1 ), indicating moderate quality of reporting.

Metabolic syndrome and risk for cardiovascular disease

Eleven studies assessed the relationship between time spent engaging in sedentary behaviour and risk factors for MS and CVD (Table 5). All of the studies reported that increased sedentary time was associated with increased risk for MS or CVD. However, the results of these studies should be viewed with caution as the proportion of children and youth who have measurable health risk factors for MS or CVD is quite low. Longitudinal studies found that those watching more than 2 hours of television per day had higher serum cholesterol levels [88] and were more likely to have high blood pressure [215] than their peers who watched less TV. Cross sectional studies reported that high levels of screen time and self-reported sedentary behaviour were associated with increased risk for high systolic and diastolic blood pressure $[47,108,216,217]$, higher HbA1 c [218], fasting insulin $[134,216]$, insulin resistance $[48,219]$, and MS [220]. These risk factors increase in a dose response manner with increased screen time $[216,220]$. One cross sectional study reported a significant relationship between watching TV and increased cholesterol in adolescents, but not in younger children [185]. The level of evidence for MS and CVD risk factors was classified as Level 3 with a mean Downs and Black score of 21.7 (standard deviation: \pm 2.1 ), indicating moderate quality of reporting.

Table 4 Summary table of results showing relation between sedentary behaviour and fitness

\begin{tabular}{|c|c|c|c|}
\hline $\begin{array}{l}\text { Type of } \\
\text { Study }\end{array}$ & $\begin{array}{l}\text { Number of } \\
\text { Studies }\end{array}$ & $\begin{array}{l}\text { Number of } \\
\text { participants }\end{array}$ & Narrative rexommendation and main findings \\
\hline FCT & 0 & & \\
\hline Intervention & 1 & 76 & Heductions in sedencary behaviour lead to increased ftness. \\
\hline Longtudinal & 2 & Sol & $\begin{array}{l}\text { Cne study showed no association whereas one study showed higher musculosbeletal finess in } \\
\text { those watching }<2 \text { trs of TV per day. }\end{array}$ \\
\hline $\begin{array}{l}\text { Cross } \\
\text { sectional }\end{array}$ & 12 & 17227 & $\begin{array}{l}>2 \text { hrs of scoeen time per day is associated with better woymax scores, better musculoskeletal } \\
\text { and cardiorespiatory fitness scores. }\end{array}$ \\
\hline $\begin{array}{l}\text { Total of all } \\
\text { studies }\end{array}$ & 15 & 17864 & $\begin{array}{l}\text { Those watching less than } 2 \text { hours of TV a day showed higher results for fichess testing and more } \\
\text { favcurable bone hearh. } \\
\text { Mean Downs and Black score }-20.6 \text { It } 2.1 \% \text {, Level } 3 \text { evidence. }\end{array}$ \\
\hline
\end{tabular}


Table 5 Summary table of results showing relation between sedentary behaviour and markers for metabolic syndrome and cardiovascular disease

\begin{tabular}{|c|c|c|c|}
\hline $\begin{array}{l}\text { Type of } \\
\text { Study }\end{array}$ & $\begin{array}{l}\text { Number of } \\
\text { Studies }\end{array}$ & $\begin{array}{l}\text { Number of } \\
\text { participants }\end{array}$ & Narrative recommendation and main findings \\
\hline AnCT & 0 & & \\
\hline Longitudinal & 2 & 1675 & $\begin{array}{l}>2 \mathrm{hr} \text { of TV per day is assodated with higher serum cholesterol levels. }>1.2 \mathrm{hes} \text { of TV per day is } \\
\text { associated whe increased systolic blood pressure. }\end{array}$ \\
\hline $\begin{array}{l}\text { Cross } \\
\text { sectionsl }\end{array}$ & 9 & 17339 & $\begin{array}{l}>2 \text { af screen time per day is axsociated wth higher blood pressure and incressed risk for } \\
\text { metabolic syndrome. }\end{array}$ \\
\hline Intervention & 0 & & \\
\hline $\begin{array}{l}\text { Total of al } \\
\text { studies }\end{array}$ & 11 & 19014 & $\begin{array}{l}\text { Increased scresen time is associated with increased risk for markers of metabolic syndrome and } \\
\text { cardiovascular disease. Fisk increases in a dose-response manner. } \\
\text { Mean Downs and Black score }=21.7 \text { \& } 200 . \text { Level } 3 \text { evidence. }\end{array}$ \\
\hline
\end{tabular}

\section{Self esteem}

Fourteen studies assessed the relationship between time spent engaging in sedentary behaviour and self-esteem (Table 6). One RCT aimed to increase physical activity and decrease TV viewing [221], leading to a trend in improvements in self-esteem $(P=0.26)$ and concerns with body shape $(p=0.03)$. Intervention studies that targeted changes in sedentary behaviour produced inverse changes in physical self-worth and self-esteem [52,54]. Cross sectional studies showed that increased screen time was associated with higher depressive symptoms, low self-esteem, and decreased perceptions of self-worth $[44,115,147,212$, 221-223]. There was evidence for a dose-response relationship as each additional hour of screen time seemed to increase the risk for lower self-esteem [147]. Two studies $[224,225]$ reported that increased TV viewing was associated with decreased self-esteem in boys but not girls, and increased aggression in girls but not boys. Two studies showed no significant relationship $[226,227]$. One study [228] showed a significant relationship between increased TV viewing and decreased self-esteem in adolescents but not in young children. The level of evidence for studies examining self-esteem was classified as Level 3 with a mean Downs and Black score of 21.0 (standard deviation: \pm 24 ) indicating moderate quality of reporting.

Pro-social behaviour

Eighteen studies assessed the relationship between time spent engaging in sedentary behaviour and pro-social behaviour (Table 7). The one longitudinal study examining the relationship between sedentary behaviour and pro-social behaviour found that sustained TV exposure (i.e. 22 hours per day) was a significant risk factor for behavioural problems [229]. Cross sectional studies reported similar findings. Those who watched less TV were more emotionally stable, sensitive, imaginative. outgoing, self-controlled, intelligent, moralistic, college bound, and less likely to be aggressive or to engage in risky behaviour $[42,115,230-235]$. Two studies found a significant relationship between increased computer use and behaviour problems in boys $[111,236]$ but not girls. One study showed that increased TV viewing was associated with aggression in girls but not boys [225]. The level of evidence for studies reporting on pro-social behaviour was classified as Level 3 with a mean Downs and Black score of 19.9 (standard deviation: \pm 1.3 ) indicating moderate quality of reporting.

Academic achievement

Thirty five studies assessed the relation between time spent engaging in sedentary behaviour and academic achievement (Table 8). Academic achievement was measured in a variety of ways but included measures of LQ. school grades, grade point average (GPA), performance on standardized tests, and self-report questionnaires (e.g. students rated their own level of academic achievement). The longitudinal studies included in this review found that children who watched higher amounts of TV had

Table 6 Summary table of results showing relation between sedentary behaviour and self-esteem

\begin{tabular}{|c|c|c|c|}
\hline $\begin{array}{l}\text { Type of } \\
\text { Study }\end{array}$ & $\begin{array}{l}\text { Number of } \\
\text { Studies }\end{array}$ & $\begin{array}{l}\text { Number of } \\
\text { participants }\end{array}$ & Narrative recommendation and main findings \\
\hline RCT & 1 & 61 & $\begin{array}{l}\text { Girls who decreased sedentary behaviour had lower booly dissarisfaction and showed a trend } \\
\text { towards improwed selfesteem. }\end{array}$ \\
\hline Intervention & 2 & 994 & Decreases in sedentary behaviour lesd to improved sef worth and selfesteem. \\
\hline Longitudinal & 0 & & \\
\hline $\begin{array}{l}\text { Cross } \\
\text { sectional }\end{array}$ & 11 & 71068 & $\begin{array}{l}\text { Those with higher reported sedentary behaviour had poorer scores on ser worth. This associanion } \\
\text { seems to increase in a dose-response manner }\end{array}$ \\
\hline $\begin{array}{l}\text { Total of all } \\
\text { studies }\end{array}$ & 14 & 72113 & $\begin{array}{l}\text { Each additional hour of TV viewing was associated with decreases in sef-worth and self-concept } \\
\text { Mean Downs and Black score }-21.0 \Leftrightarrow 2.4 \lambda \text { Level } 3 \text { evidence. }\end{array}$ \\
\hline
\end{tabular}


Table 7 Summary table of results showing relation between sedentary behaviour and pro-social behaviour

\begin{tabular}{|c|c|c|c|}
\hline $\begin{array}{l}\text { Type of } \\
\text { Study }\end{array}$ & $\begin{array}{l}\text { Number of } \\
\text { Studies }\end{array}$ & $\begin{array}{l}\text { Number of } \\
\text { participants }\end{array}$ & Narrative recommendation and main findings \\
\hline FCT & 0 & & \\
\hline Longitudinal & 1 & 2707 & Wasching $>2$ hrs of TV per day is a risk factor for social bethaviour problems \\
\hline Intervention & 0 & & \\
\hline $\begin{array}{l}\text { Cross } \\
\text { sectionsl }\end{array}$ & 17 & 91934 & $\begin{array}{l}\text { Individuals wanching }>3 \text { hrs of TV per day are more libely to exhibit poor sodial behawours and be } \\
\text { more aggressive. Limited evidence to suggest this relationship is stronger in bays. }\end{array}$ \\
\hline $\begin{array}{l}\text { Total of al } \\
\text { studies }\end{array}$ & 18 & 94391 & 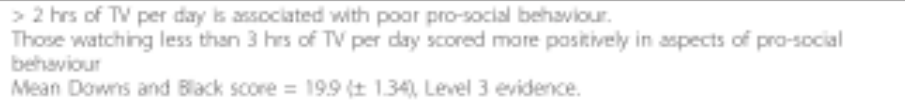 \\
\hline
\end{tabular}

greater difficulties with attention as teenagers [41], showed lower progression for reading level [237], and performed worse on cognitive tests [238] than those watching less than one hour of television per day. The majority of cross sectional studies (75\%) reported that children and youth who watched higher levels of TV tended to spend less time doing homework, studying, and reading for leisure which may lead to a decrease in academic achievement $[42,181,239-255]$. This association increased in a dose response manner [181,244,248]. Ten of the cross sectional studies found no significant relationship [57,226,227,238,256-261], One study [228] found that this relationship was significant in adolescents but not younger children. The evidence for academic achievement was classified as Level 3 with a mean Downs and Black score of 19.2 (standard deviation: \pm 2.1 ) indicating moderate quality of reporting

\section{Quantitative data synthesis}

Data for each of the outcomes were assessed to determine if they were sufficiently homogeneous to make meta-analysis appropriate. The only outcome for which data were consistently collected and reported and for which the characteristics of the studies were similar enough to undertake a meta-analysis was body composition. However, this was only for the RCTs; the longitudinal, cross sectional and intervention studies that examined body composition had too many inconsistencies to allow for a quantitative synthesis of results.
Change in mean BMI before and after the intervention (at the longest point of follow-up for each study) was used as the point estimate for the meta-analysis of the RCT data. Of the 8 RCTs, only 6 had data that could be used to calculate the change in BMI after the intervention $[50,58,221,262-264]$ (the other two reported on prevalence of overweight and obesity) [57,265]. Of the remaining six studies, one [50] examined standardized estimates of BMI only and one [262] presented only medlan change in BMI and not a mean change. Study authors were contacted for missing information, but no additional data was made available and thus these studies were excluded from the meta-analysis. Meta-analysis of the 4 RCTs that remained revealed an overall significant effect of $-0.89 \mathrm{~kg} / \mathrm{m}^{2}(95 \% \mathrm{Cl}$ of -1.67 to $-0.11, \mathrm{p}=0.03$ ) indicating an overall decrease in mean $\mathrm{BMI}$ associated with the interventions (Figure 2). The Chi square test for heterogeneity was not significant but the $\mathrm{I}^{2}$ was $46 \%$ indicating that there was low to moderate heterogeneity in the data. The funnel plot showed no indication of publication bias (data not shown).

Meta-analyses were not undertaken for other outcomes or study designs because there was substantial heterogeneity in the units of measures and type of reporting of sedentary behaviour, as well as the specific measures of each outcome. For example, when reporting on the relation between time spent watching TV and overweight and obesity, one study may report the relation between

Table 8 Summary table of results showing relation between sedentary behaviour and academic achievement

\begin{tabular}{|c|c|c|c|}
\hline $\begin{array}{l}\text { Type of } \\
\text { Study }\end{array}$ & $\begin{array}{l}\text { Number of } \\
\text { Studies }\end{array}$ & $\begin{array}{l}\text { Number of } \\
\text { participants }\end{array}$ & Narrative recommendation and main findings \\
\hline RCT & 0 & & \\
\hline Longitudinal & 3 & 3530 & Watching $>1 \mathrm{hr}$ of TV per day is associated with attention difficulties. \\
\hline Intervention & 0 & & \\
\hline $\begin{array}{l}\text { Cross } \\
\text { sectional }\end{array}$ & 32 & 157637 & $>2 \mathrm{hrs}$ of screen time per day resulted in lower academic achievement. \\
\hline Intervention & 0 & & \\
\hline $\begin{array}{l}\text { Total of al } \\
\text { studies }\end{array}$ & 35 & 161167 & 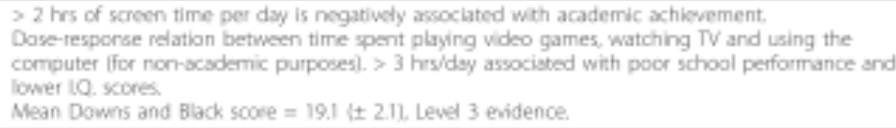 \\
\hline
\end{tabular}




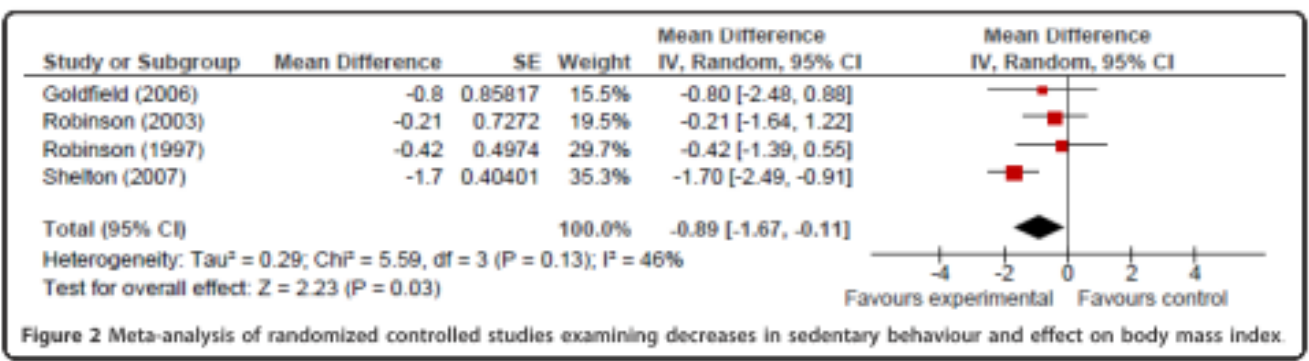

the frequency of TV watching and skin fold thickness, whereas another may examine the relation of daily volume of TV watching and BMI. Even for studies that examined the same outcome, for instance BMI, some would report the proportion overweight or obese, while others would report mean BML. In addition, some studies reported on data for males or females only, while others reported only overall estimates and many were missing key information about participant characteristics or study design. As a result, we were unable to determine common point estimates and associated measures of errors for many of the studies. Due to the scope of the review, it was not feasible to contact every author for individual data to re-run the analyses. Developing reporting standards for primary studies examining the relationship between sedentary behaviour and health would help to ensure that appropriate data are available for future meta-analyses.

\section{Discussion}

Based on this systematic review of 232 studies, sedentary behaviour (assessed primarily through increased TV viewing) for more than 2 hours per day was associated with unfavourable body composition, decreased fitness, lowered scores for self-esteem and pro-social behaviour and decreased academic achievement in school-aged children and youth (5-17 years). This was true for all study designs, across all countries, using both direct and indirect measurements, and regardless of participant sample size. All studies examining risk factors for MS and CVD disease reported that increased sedentary time was associated with increased health risk; however, the included studies examined a wide range of risk factors, and thus there was insufficient evidence to draw conclusions on the relationship for metabolic risk as a whole.

High heterogeneity of the included studies limited meta-analysis to RCTs examining the relationship between television viewing and BMI. This revealed a trend to support the hypothesis that decreased time spent sedentary is associated with decreases in BMI. This result should be interpreted cautiously, given that it is only based on a small number of RCTs and that only half of the RCTs included in the review were included in the meta-analysis. Nonetheless, this meta-analysis of RCTs, which are considered to be the highest quality of research evidence, coupled with the qualitative syntheses of data from the other study designs, provides consistent evidence of the inverse relationship between sedentary behaviour and health outcomes, and that reducing sedentary behaviour can improve body composition. Furthermore, this finding was consistent with the results of observational studies and previous reviews $[19-21,23,25]$.

Studies included in this review used primarily indirect measures (i.e. parent, teacher, and self-report questionnaires) to assess time spent engaging in sedentary behaviour. Those studies that did use direct (i.e. accelerometer) measures found that children and youth are spending a large proportion of their day (up to 9 hours) being sedentary $[24,27,29,39-47,49,178]$. Therefore, for some children and youth, a viable approach to improving health may be to work towards a reduction of at least some of their sedentary behaviours either through smaller, micro-interventions (e.g. interrupting prolonged sedentary time), or lager macro-interventions (e.g. population-based interventions and public health initiatives). Decreasing sedentary time is important for all children and youth, but it may be may be especially important to promote gradual decreases in the most sedentary group as a stepping stone to meeting sedentary behaviour guidelines [266].

\section{Strengths and limitations}

Strengths of this review included a comprehensive search strategy, a-priari inclusion and exclusion criteria and analyses, and inclusion of non-English language articles. We included direct and indirect measures of sedentary behaviour and focused on 6 diverse health indicators in children and youth. Although efforts were made to include grey literature (e.g. by contacting key informants and reviewing government documents), we did not include conference proceedings and other types of grey literature because it was impractical and unfeasible to sift through all unpublished work, and also because of limitations in 
the quality of reporting in conference abstracts $[267,268]$. We do not anticipate that additional, unpublished work would change the results.

Our study has limitations, including the types of outcome measurements and analyses reported in the primary studies and primary study quality. The scope of this review was large and included a great deal of health indicators and measurement tools. A more detailed metaanalysis would have allowed us to estimate the overall effect sizes for each outcome. However, due to the heterogeneity of the data, it was impossible to complete such analysis. Furthermore, some studies had missing information on participant characteristics making it impossible to determine if basic demographics act as a confounder for the relationship between sedentary behaviour and health. Many studies also grouped their variables into tertiles, or groups that also took into account physical activity level. Although it was still possible to ascertain information regarding the association between level of sedentary behaviour and health indicators, it made it very difficult to compare the information across studies. Similarly, very few studies measured time spent being sedentary directly (i.e. with direct observation or accelerometry). Previous work $[269,270]$ has shown significant differences between direct and indirect measures of physical activity; similar work needs to be completed with respect to sedentary behaviour to gain a better understanding of possible biases in previous studies. Indirect measurements of sedentary behaviour often lead to grouping for analyses. This may lead to bias in the results of the systematic review as many studies arbitrarily grouped their participants as "high users" if they watched more than 2 hours of television per day. This could perhaps be falsely leading us to conclude that 2 hours is the critical cut-point or threshold. Further work using direct (i.e. accelerometer) measures of sedentary behaviour and screen time as continuous variables will help to clarify if a cut-point of 2 hours is in fact biased.

The final important limitation of this review was the type of primary studies that were available for analysis. Studies with small sample sizes were excluded; however we do not believe that this had a significant impact upon the strength or direction of associations observed in this review. The majority of studies (78.4\%) included in this review were cross sectional, observational studies, using indirect (i.e. parent-, teacher, or self-report) measurements of sedentary behaviour. Cross sectional data make it impossible to infer causation and results should therefore be interpreted with caution. However, it should be noted that due to ethical considerations, it may be impossible to conduct a RCT on the effects of long periods of sedentary behaviours in children and youth. Due to the large and diverse sample sizes available in populationbased cross sectional research, and given that this information demonstrates similar trends as those seen in RCTs and intervention studies, we believe that the evidence presented in this review provides important insights into the relationship between sedentary behaviour and health outcomes in school-aged children and youth.

\section{Future work}

The purpose of this review was to provide an evidence base to inform clinical practice sedentary behaviour guidelines for children and youth [266]. Future work is needed to translate this information into clinical practice guidelines and disseminate this information to health care providers and the general public. While this review was limited to children and youth, similar work is needed to inform sedentary guidelines for young children aged 0-5 years, adults, and older adults.

As the accessibility and popularity of multiple forms of screen-based technology increases among the pediatric population, future work needs to continue to focus on media engagement. Specifically, with increasing popularity for hand-held, portable devices, 'sedentary multitasking' is becoming increasingly common. Children and youth are able to watch television, talk on the phone, and use the computer at the same time. This is a relatively new phenomenon and we are currently unaware what, if any, are the health effects associated with this high level of 'multi-screen' time. This is also true for the effect of advancements in technology and their associated health effects. For example, 'active video gaming' (e.g., Nintendo Wiirs, Microsoft Kinect ${ }^{\mathrm{TM}}$, Sony's Playstation Move ${ }^{\mathrm{TM}}$ ) is advertised as an effective mode of physical activity. Although it is true that some games can require sufficient energy expenditure for health benefits [271], the socio-cognitive and physiological aspects of remaining indoors for long periods are unknown. Furthermore, children and youth can learn quite quickly how to use minimal gestures (e.g., using wrist movement only) to play the game thereby substantially reducing energy expenditure.

Finally, as described above, the vast majority of the current evidence has been based on self-report questionnaires focused on TV viewing and body composition. It is now clear that these two variables are related. Future work needs to move beyond this relationship and focus on other modes of sedentarism (e.g, prolonged sitting, passive transport) and other associated health indicators. To do this, objective measures of the time, type and context of sedentary pursuits will be needed in combination with robust and standardized measures of health indicators.

\section{Conclusions}

Physical inactivity and sedentary behaviour are pervasive and persistent public health challenges to overcome. This 
review demonstrates that there is a need to advocate for increases in physical activity AND decreases in sedentary behaviour. It is believed that a multi-level, multi-sectoral approach is required for this to be successful [11]. Ulti. mately, resolving the problem of inactivity requires a sustained change in individual daily activity and sedentary patterns. From a public health perspective, a reduction in sedentary behaviour may be easier than increasing physical activity per se because there are fewer restrictions (i.e. no need to change clothing or use special equipment), and can be easily attained with minimal burden to a person's time or financial resources.

This systematic review summarizes the current evidence examining the relationship between sedentary behaviours and a series of health indicators. It was determined that increased sedentary time was associated with negative health outcomes in both boys and girls: this was true across all study designs with the majority of studies $(85.8 \%)$ reporting similar relationships. The majority of current work has focused on television viewing and body composition and suggests that children and youth should watch less than 2 hours of TV per day during their discretionary time. Furthermore, chil. dren and youth should try to minimize the time they spend engaging in other sedentary pursuits throughout the day (e.g. playing video games, using the computer for non-school work or prolonged sitting). This work can be used to inform the development of evidence. based sedentary behaviour recommendations for children and youth.

\section{Additional material}

Additional file is Search strategy.

Additional file 2: Search strategy.

List of Abbreviation

BME Body Mass index; CVD. Cardiovasoular dkease; DXA or DEXA: Dua energy x-ay absorptionetry, MS: Metabolic syndrome; RCT: Pandomibed controled triat Ty: Television.

Acknowledgements

The aluthas corcrbutions to this proiect.

Michelle Kho is funded by a Felowship hawad and Bsty Prize from the Ganadian Institutes of Heath Acseanch. Travis Ssunders 15 supported by a

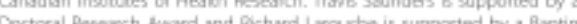

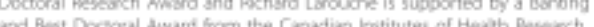
Partal finoing for the complation of this revisw came from the Puklic

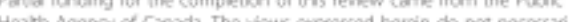

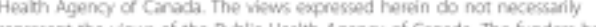

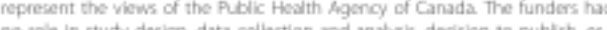
no nole in study design, data collection and analysis, dedision vo publish, of preporation of the monuscript.

Author details

'Healthy Adtive Lining and Cbesity Pesearch Crildren's Hospial of Eaghem

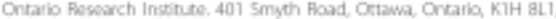

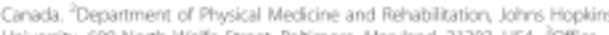
Universty 600 Nonth Wofe Street, Batimore, Maryland 21202 USA. "Office of the Task Force on Preventive Heath Care, Public Howith Agency of Canada. TBS Carling Avenue, Ottawa, Omtaria, K1/A OKS, Canacis

\section{Authors' contributions}

MT was resocrsible for the initiation conceptualestion and sesign of the systemaric review; owersaw the dara colkection and extraction, andissis, and incerpretarion of data and wis nerponsible for revking the manuscript

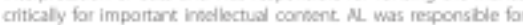
conducting the seasth data collection and exteratica, the titk of bis sasessment, snabsis and imerpretation of dota, and drafing the manuscript Masessment, andyse and imterpretation of dacs, and cratting the manusci Mek was rexponstbe for the desgn and methodaiogy of the rewew and

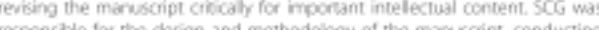
rexponste kar tore design and methodalogy of the marusoipt, condusing the meta analysh, and revking the manuscript crically for imporant ineflectual content. AC, GG, TS and AL were nesponsible for data collection and extraction, nisk of bis assessment, and were resporable for nevising the manuscript critically for important intelectual contert. JM was responsble for the generation of syslematic review search terms. MS was responsible fic mechodology of the review. All authors have read and approved the fnal manuscrion. MT ks the guarantor of the paper.

\section{Competing interests}

All suthors receved partial financisl support from the Public Health Anency of Canads no other competing imterests exist.

Hecpived 8 Apra 2011 Accepted: 21 September 2011

Published. 21 September 2011

References

1. Jenssen L, LeElani AGG Systematic review of the health benefits of prysical activity and fitness in school-aged children and youth. in

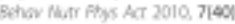

2. Parenson D. Warbution D. Physical activiny and functional limitations in odder adults: a systematic review related to Conodr's Physical Activty Guidelines int $/$ Benov Nut Phys Act 2010, 7 (38)

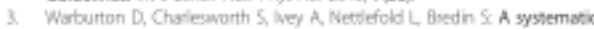
review of the evidence for Canado's Prysical Activity Guidelnes for

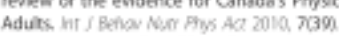

4. US. Dopartmem of Heath and Human Services. Physical Activity and Health: A Heport of the Surgeon Generat. Arlanaz, Geovgiz US Deportment of Hedth and Human Sevvices, Centers for Disease Control and Prevention, Nastional Center for Oronic Cbesse Prevention and Health homotion 1996

5. World Heath Orgarization: Globol recommendations on physizal activity

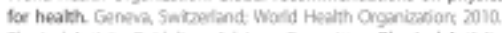

b. Physical Activity Guidelnes Advisony Committree Ptrysical Activity Guidelines Advisory Comminee Heport Washington, DC. US. Departmert of Hedth and Humsn Services; 200 e

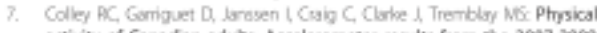
activity of Canodion adulk: Accelerometer resilts from the 2007-2009 Canadian Health Measures Survey, h Hedloh feco Volume 22 Statistic: Canada Carilogue no. 82:003) xPE; 2011111

B. Colley AC, Gamiguet D, tarseen L Craig C Clarbe 1 Tremblay MS Ptrysical activity of Conadian children and youtts Accelerometer results from the activity of Canadian chidren and youth Acoelerometer results from the 2007-2009 Cansdian Heath Messures Survey. I k

9. Samarak PT, Tremblay M5. Limitaicons of Carada's patysical activity dato implications for monitering trends. Appl Fhysol thitr Merob 2007, 32 5185.5194.

10. Shields M, Tnemblay MS, Lavidette M, Caig C, Lrasen L, Connor Gorber ? Fitness of Canadian adults: resuts from the 2007-2009 Cansolisn Heath

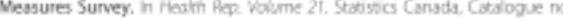
82-003-TPE: 201011

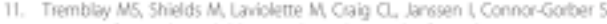
Fitness of Caradian Chidren and youthe Alesults from the 2007-2009

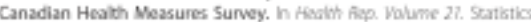

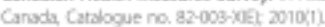

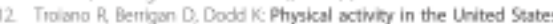

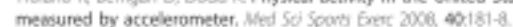


13. Flegal kM Comcl MO. Oguen C. Curin Lit Previlence and trends in

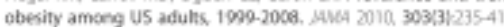

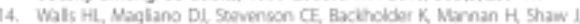
Peeters A Projected progression of the prevalence of obesity in Australa. Obesty (Shive Springl, Epub 2010 Dec 13

15. Obhanshy SL, Passaro DL, Hershow PC, Layden I, Cames BA, Brodt l, Haphick L

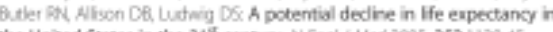
the United Stanes in the $21^{\alpha}$ conary. $N$ Engl I Med 2005, 352:1130-4!

16. Tremblay ME. Warbunon DEFl lansen L Pafterson DH Latimet AE. Fhodes FE, Kho ME, Hids A, Leflianc MG, Zehr L Menumets K, Duggan N.

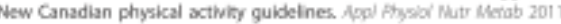
3asesen

17. Bul FC. Expert Working Groups Ptysical sctivity guidelines in the UK: Review and recommendations. Leicesserstive, UK: Schood of Sport.

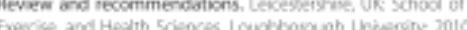

18. Oieky AD, Salmon I Tros SG. Hiniley T. Disoussion paper for the development of physical activity recommendations for chiddren under 5 years. Australin Govemment Department of Heath and hgengy 2006

19. Active Heditty Mids Ganodx Healthy habits start earlier than you think Aeport Card on Physical Activity for Children and Youth. Torontox Adtive Heartry Kied Canada: 2016

20. Adtive Hedithy Wids Canadic Active kids are fis to leam - Report Cand on Physical Activity for Children and Youth. Tosonto, Active Heathy Kics Canada; 2009

21. Acthe Heditty hads Ganadx lit's tome to unphug our kids - Report Cand on Ptrosical Activity for Children and Youth, Toxretos Active Heglthy Kind Canada: 2009

22. Marthews CE, Ghen KY, Freedson P5, Buchowdi M5. Beech BM, Pane AR Troino F. Amount of time spent engacing in sodencary behawiours in

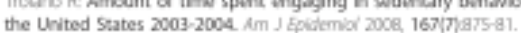

23. Aideour VI. Foetr UG. Aloberts DF: Generotion MZ Media in the Lives of 8- to 18-Year -oids. A. Kaber Family Foundation Study. Merlo Park Falforris: Henty L Kaser Family Foundation: 2010 .

24. Whitt-Gover MC Tajor WC. Fiopd MF, Yone Mu, Yancey AK, Matthews CE Disparties in physical activity and sedemary betwrioes among US children and adolesents! prevalence, coriciaces, and imerverto on implications. I Publi: Heath Palky 2009, 305 suppl 115309-34.

25. Wrk SPL, Penney Th, Langlle ll: The relationship between screen time, physical activity, detary intake and hed thy weights in children and youth: literature review and recommendations for intervention. Haroor Halrax Regional Physical Activity and the MK. Hedth Cente. Nova Scotia Doparment of Hoath Fromotion and Protoction zopo

26. Whitbock EP, OConnor EA. Wilams SB, Beil Th, Lutz KW. Effectweness of weight management imterventions h chidrerc A targeted systematic review for the USPSTE. Andings 2010,125 tos-418.

27. Treuth MS, Beggett CD, Prott CA, Going SB, Elder D. Oremeco E. Vebber $L S$ A bonghudinal study of sedencary behwior and everweigh

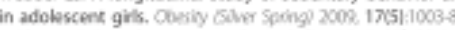

28. Leelanc MG, Saunders TI, Larouche R, Colley FC, Golafield G, Toemblay ME Helasion between sedentary behwrours and health outcomes in chidren and youth. bour Meeting of the North American Sockty for Pedlank Evercie

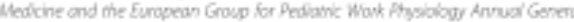
Meening 2010 Ses 22-26, 2010, Niagara-on-the Late, ON. (obstiacti.

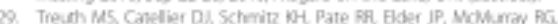
How FM, Yang S. Wobber L Woekond and weekday pattems of physical sctivity in ovenweight and normal-weight adolescent girls. Dbesty istiver Soung $2007,15071782-8$

90. Katomorak PT, Onurch TS, Craig O, Bouchard C. Sitting time and mortality from al causes, cardionsscular disease, and cancer. Med So Spovers Exert 2009 . 41996-1005

31. Omen N. Bauman A. Brown W. Too mach sitting: a novel and importart predictor of chronic dissase risk? Br I Sponts Mert 2009, 43(2) B1-3.

32. Tremblyy MK, Colcy R, Suinders TL, Hedty GN. Owen N: Physiological and heath implications of a sedentary lifestyle. Apd Physiol Nutr Metab 2012 $35: 725-40$.

33. American Academy of Pediasics: Active heathy living Prevertion of

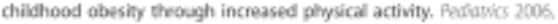
$117 \mid 5 \times 1834-42$

34. Canadian Pzediaric Society, Hed thy Active Living Committee. Healthy

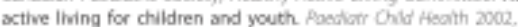
$7 \times 39-45$
35. Donems SH. Black N- The feastility of creating a checkist for the assessment of the mothodological quality both of randomized and non-

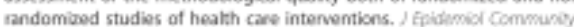
thateh 1993 , 52.377 .84

36. Lau DC, Douketis ID, Morison nM, Hramiak HU, Sharma AM, Ur E 2006 Cinical practice guidelnes on the management and peevention of obesity in sodults and children (summary] Covi 2007, 17608151-13

37. Desimarian R Laid N Meta-analysis in dirical triak Contro' on Thials $1966.7: 177-88$

38. Hiogins JPT, Thompocon SG Quantifying heterogeneicy in a meta analysk. Star Med 2002, 21:1539:54

39. Lampert T, Sygusch A, Sctlack A, Lampert T, Sipgusch R, Schibok R, Uuse of electronic medis in adolescence. Pesults of the German Hedith interview and Eamination Survey for Children and Adolescents [KGGGS],

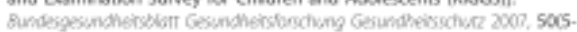
6) 643.52 , German.

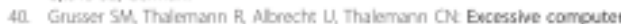
urage in adolescents - A psychometric evaluation. Wener Bibche

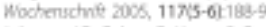

41. Jotroon JG, Cohen P, Kasen S, Brook J6. Extensive telerision wiewing and the development of attertion and leaming diffoulties during adoleksence. Anch Pediay Adolex Med 2007, 161 (5) 460 -6

42 Ormert E, Toyran M, Yurctuock K, Oamert E, Tayran M, Yundabie K Behaviord contiates of teievision viewing in peimary school children evaluated by the child behavior checklist. Anch Pediat Adolesc Mad 2002 $15691910-4$

43. Neumam H. Neumam P. Nutrition and euestise of schookctiktren. Results of a cross-sectional study at the Lessing Gymnasium tsecondary) school in Manerioim, Germany? Desch Mod Wecherich 2007, 132151. $5212736-42$, Geman.

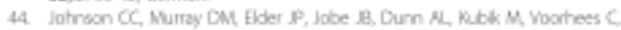
Sctuscher $K$. Depressive symptoms and physical activity in adolescent girls. Mod So Sports Ever 2008, 40157818-2t

45. Nogueira IA edo da Costa TH Nogueira JAR Macedo da Cosa TH Gender dfferences in phrysical activity, sedentary behavior, and their retation to body composition in active Brasian adolescents. J Fhy Ad heath 2009 (1) $93-8$.

40. Poshitt EME: The evolution of childhood obesity. in $J$ Aedvit Cbes 2010 . Sisuped 11:11.

47. Sugjami T, Xe D, Gramam-Mar RC, hove K. Kobapasi Y, Seetler Ns Diecary and liestyle factors associned with blood pressure among US

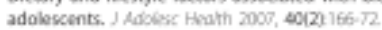

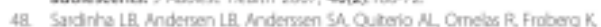
Aaddoch $C$, Elebund U Oejectively mexured time spent sedentary is associsted with insulin resibtance independent of overdl and centsal body fat in 9- to 10-year-old Portuguese children Didele Cave 2006, 3163569-?

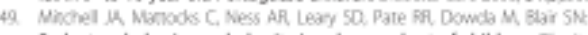
Sedentary behavior and obesify in a large cohort of dildken, Obesiy Esler Springl 2009, 17\%ay.1596-602

50. Epstein LH, Aoemmich IN. Alobirson IL, Paluch RA, Wriswicz DO Fuecth $H$, Aobireon Thk A randomized trial of the effects of reducing television viewing and computer use on body mass index in young

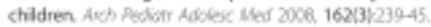

51. Samon 1 Ball K Hume C Booth M, Crawlond D- Outcomes of a group randomizod trial to prevent excess weight gain, reduce screen behwiours and peomote physical activity in 10-year-old children: switch. play. ine J Obes flond 2003 32/4|1301-1?

Q2. Goldfeld GS, Malory R, Porbe T, Cunningham T, Lego C, Lumb A, Parker K. Prufhomme $D$, Adomo NB: Effects of modfying physical axtivity and sedentary behwior on psychosocial adustment in overweightoblese children I Pediar Pgychol 2007, 32177783.93.

53. Ochoa MC, Moreno Alaga M, Martinez Gonvalez Ma, Marthez IA, Marti A Prediktor factors for childhood obesty in a Spanish case-contsol study. Numtion 2007, 23/5;3798.

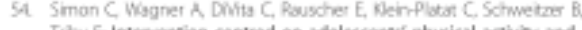
Triby E Imterention centred on adolescents physical activity and sedentary behawiour 0CAPS: concept and 6-month results. hit f Obes Relat Mrrab Disond 2004 2815ippl 31596:5103.

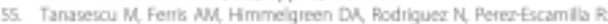
Bobehavioral factors are associsted with obesity in Puerto Rican children I Nut 2000 13007:1234-42 


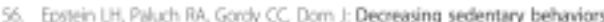

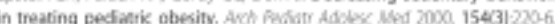
57. Gortmabar SL, Penersan K. Whacha L Sobol AM Deer S, Far MK, Laird N Reducing obesiby via a school based interdisciplinary intervention smong youth: Planet Heaith Anch Peaturn Adales Med 1999, 153101009-18.

58. Aobiroon The Aeducing children's television wiewing to prevent obesity a randemined controlled trial Jus $1999,262[16 \mathrm{t}-1561-$ ?

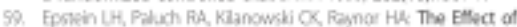
Reinforcoment or Stimulus Control to Foduce Sodenary Bchwior in the Trestment of Pediatric Cbesity. Hesih Pijechol 2004, 23(4) 371-BC

60. Epsich LH, Aoemmich W, Paliuch AA, Raynor HA: Physical activity as a

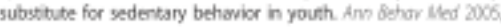
290012009

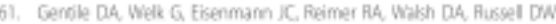

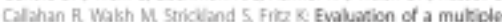
ecological level child obesity peewention program: Switch what you Do, View, and Chew. buc Mer 2009,749

02. Harison M. Bums CF, MoGumess M, Mesin l, Murphy Mat Influence of a heath education intervention on physical activity and screen time in

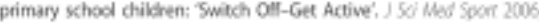
9:51388.94

63. Pusber LR. HIIC S Suton 1 Cosder $K$, Wardle $\perp$ Defferences in ptrsical sctivity and sedentary time in relation to weight in 8.9 year old children. int J Behor hutr Phys Act 2008.5167 .

64. Hespeth $\mathrm{K}$. Carin L, Wase M. Cranford D. Predictors of body mass inde: change in Austrdian primary schod childen, hit $f$ Pediar Obes 2008 $4 / 1 / 45-53$

65. Timperio A Samon 1 Ball K, Bayr LA Telford A, lackoon M Salmon L. Crowford D. Family physical activity and sedentary enweronments and weight change in childen, ht J Pedigri Ohes 2008 , 3138180-7.

60. Darre AN A national longitudinal study of the sssocistion between hous of TV viewing and the tajectory of BMI growth among US

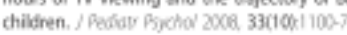

67. Henderson ve Langitudinal asvociations beoween telovision viewing and body maxs index among white and black girls. I Adoksc hooth 2007 $41(6)-544-50$

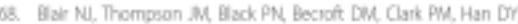
Adbrom E Walde KE. Wid C. Mitchell EAC Risk factors for obesity in ?. year-dd Europen children the Aucllend Birthweingt Collaborative Shudy. Anch Dis Ond 2007, 920106866-77

69. OBrion M Nader PR, Hous FM, Bradloy R, Friedman S1, Bokiky 1 Susman E The ecology of childhood overweight a 12 -year longitudinal analyss. $h$ J Ober fland) 2007, 31/9/146\%-78.

70. Gatele $S$, Chang $Y$, Krul $\ell$ - Television watching and frequency of fomity meab are predictive of overweight anset and persistence in a national

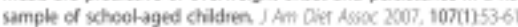

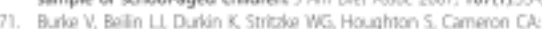
Telovision, computer use, physical activity, diet and fooness in Australian

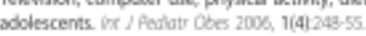

72. Hancos RL, Poutton R. Watching television is associsted with chlidhood obesity: but is it divically important? ine I Obes flond 2006.3011:171-5.

13. lane KF, Bums TL. Levr SM kowa Bone DS. Tracking of activity and sedemary behwioss in chidhood: the lowa Barw Develagenent Studs. Am L A fiv Mod 2005, 2913) 171 \&

74. Parsons TL, Power C Munor $O$. Physical actwity, telerision viewing and body mass index a cross-sectional andysis from chilchood to

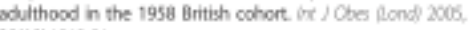
$29010+1212-21$

75. Kax H, Owoi W5. Mapo MS. Haris Kt Daration of televisien watching is

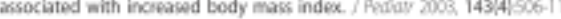

76. Berkey CS, Rodvent HR, GIIman MW, Coldtz GR. Oneyear changes it sctivity and in insctivity among 10- to 15 -year-oid boys and girts: relationship to change in body mass index. Pertative 2003, 111 (4)

37. Phorgews A, Joliffe D, Howad LL: Socioeconomic, behavicural and etwinomemal foctors predicted body weights and household food insecurity scores in the Early Chidhood longtudinal Spuby.

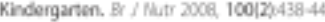

78. Chen 1 , Wal D, Kennedy C, Unrithan V, Yeh OH: Predictors of increased body mass index in Chinese children. Alog Condonox . Nurs 2007. $2203139-4$

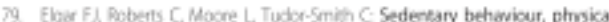
ativity and weigh problems in adolescents in Wales. Pubci Healon 2005 11966151824

50. Hesketh $\mathrm{K}$, Wake $M$, Graham M, Wavers E Stability of televkion viewing and electronic game/compuser use in a prospective cohort study of Australian children: Relstionshio with body mass index int I lechov Nur Plys Act 2003, 4600

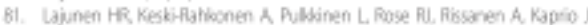
Leisure activity pateems and their asseciations with everweight: a

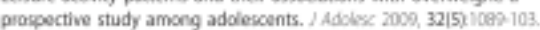

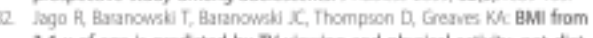
3-6 $\mathrm{y}$ of age is predicted by TV viewing and physical activity, not det. int J Ober thand 2005, 29/6)557-64.

63. Aires L Andersen Lit, Mendonca D. Martino C. Stha G Mota k: A 3-year lenghudinal andlysis of dharges in forwess physial activing, Gatness and

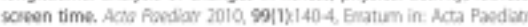
$2010.99(1) 153$

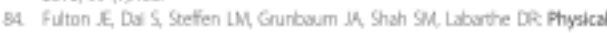
activity, energy intake, sedentary behavior, and adposity in youth. Am

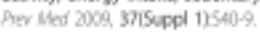

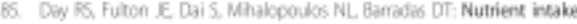
olvysical activity, and CVD risk factors in dildken: Proipct HeartBeat! Ant

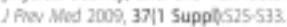

97. Borradale KE, Foter GD, Mor H. Karpyn h Greman S Gundy K Nachmsn $\perp$ Vander Weu S., Bonuch BF: Assoolations between the Youth Adolescent Questionnaire, the Youth/Adolescent Activity Questionnaire. and body mass index $z$ score in lowincome inner <ily fourth through sixth gade chidren. Am J Con Matr 2006 B7/60 $1650-5$

87. Must A, Handini LG, Tycor D. Philips 5M, Naumova EN, Diez WH Activing. (nsctivity, and screen time in relation to weight and fatness owes

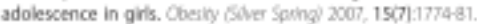

6y. Hisncox RI. Mine BI. Poulion R. Association between child and adolescent televisian viewing and adut hedith: ol longtudinal birth cohort study. Lence 2004, 36404300.257 62

62. Maffés $\mathrm{C}$ Talamini $\mathrm{G}$. Taro Linflience of diet, physical activity and parents' obesity on children's salposity a fouryear longinudinal study. int J Ober fiolax Metab Olsord 1998, 22|15:758-64.

90. Bewer CS, Aockest H. Feld AE, Gliman MN, Frasier AL, Camargu Ch t. Coldtz Gat Activity, detary intake, and weight changes in a longinudines Gudy of preadolestert and addiescent beys and give. Pedianio 2000 $105 / 41+56$

1. Sun Y, Setine M, Kayamimori S: Lfestyle and owerweight among apanese adolescentas the Toyama Binth Cohort Study. I Enidemilal 2009 $19|6| 303-10$

92. Lajous M. Onewsmo I, Pesergon KE, Hemander frodo B., Gruz Waldez A. Hernendez.Avia M, Laccano Ponce E: Screen time and adiposity in adolescents in Mexico. Pude Heath Muty 2009. 12110-1938-45

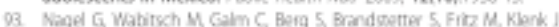

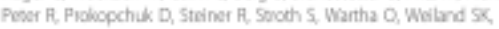
Steinacker $\perp$ Deberminants of obesity in the Ulm Research on Metabolim, Exercise and Lifestyle in Chidren (UAMELLCCE, EW J Pediort 2009, $168210 \times 1259-6 ?$ ?

94. Morgenstem M Sament JD. Henewirbel R Melation between socioeconomic suatus and body mass index: evibence $\alpha$ an indrect

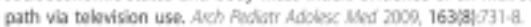

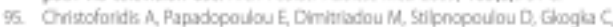
Katrus G, ThanassioutMetras $M$ : Aeference values for quancistive utrasonography (QUS) of radius and tibis in hesithy Greek pediatric

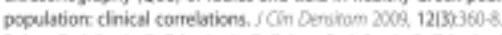

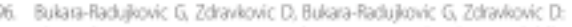
Pysical activity as an importank decerminant in derveloping childhood obesty. Mad Fugr 2009, 6213-4;:107-13.

97. Voder AM Watters ST. Hantik TR. Hoebcher CMt Television viewing and rocking behwions of fourth- and eighth-gade schookchidren in Teass. Per Oronic Des 2009, 6IStehe9.

96. Steflen LM, Cai S, Fullon J, Labarthe CA Overweight in childen and adolekents assodated with TV viewing and paremal weight: Projed

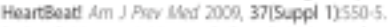

92. Padez C, Mouvo (, Moreis P. Aorado V. Long skep duration and childhood overweightiobesity and body fat. Am I Human flol $200 \mathrm{~s}$ 211303716 
100. Wong SL Leathendale ST: Association between sedentary behavior. phrsikal activily, and obesity; inactivity among adtive kids. Fev Chor Dis 2000,611$) 426$

101. Pratt C, Webber LS, Baggeet CD, Ward D, Fare Fr, Loham T, Litie L, Elder ff Sedentary activity and body composition of middle school girls: the trial of activity for adolescent grils. Afes O Exer Spont 2008, 79/4 458-0?

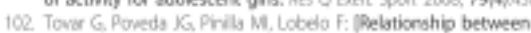
overweight, physical activity and physical fitness in school-aged boys in Bogoca Colombial, Anch Lashoam Ne.r 2008, 58/31265-73, Sparish

103. Anderson SE, Econamos CD, Must A. Active play and scroen time in us children aged 4 to 11 years in relation to sociodemographic and weight status characteristics: a nationally representabive cooss-sectional anablysa. BMC Public Heath 2008. S006.

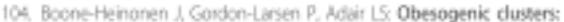
mulbidimensional adolexcem oberitymelated behaviors in the U.S. Am Brion Med 2008, 36/37217.32

105. Beyerieh A, Fahmeir L, Mansmann U, Toachin AM Alternative regression models to assess increase in childhood BML BMC Med fes Methodol 2008 BSS

106. Singh GX, Kogan MQ, Van Dyck PC. Sahpush Mt Racialiethric. sociceconomic and betwioral determinants of childhood and adolescemt obesity in the United Stanes: analyzing independent and

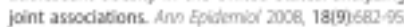

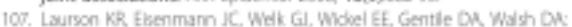
Combined influence of physical activity and screen time recommendstions on childhood overweight $f$ Pediar $2008,153(21209-14$

106. Wels X, Hold PC Reichert FF, Moneces AM Aeayo CL. Wolora CG Steep paterers and television verwing in relation to obexity and blood pressure: evidence from an adolescern Brasilan binth cohart. hit 5 Obes (lond) 2008, 32:77):10429.

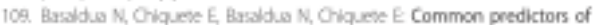
excessive adiposity in childten from a region with high prevalence of

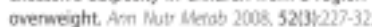

1 la vicente-sodiguez G Rey-Lopez P. Martin-Matlas M Moreno L Wamberg l Fedondo C Texcedor P, Delgado M Mracos A Cascilo M B.eno M AVENU Study Gnoup Telovision watching, videogames, and excess of body fat in Spanish adolescents: The AVENA study. Netribor $2006,2417-5,0054-02$.

111. Lestherdsk ST. Wong SL. Modifisble characteristics associsted with sedentary behwiours among youth. hit / Peviofr Obes 2009 . 3/2093-101

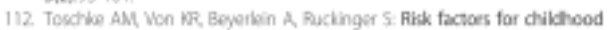
obesity! shift $\alpha$ the entire BWI distribution w. shift of the upper tal only in a cross sectional study. auc Pubic Healh 2008, 8.115

113. Elsermann XC, Bsrtee RT, Smath DT, Welk GI, Fu Q Cambined influence of physical sctivity and television viewing on the risk of overweight in US youth, hy J Ooes Itond $2008,3214 \times 513-9$

114. Miffeis C Gezzani A Pencone L Del Gualke EM Saggree G Taro L Could the savory tasse of seacks be a further risk factor for orerweight in

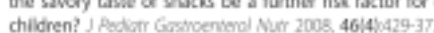

115. Chen Mr, Lou 'M Wu NF' The relationship between TV/computer time and adolescents' health-promoting behavior: a secondary data anstyys. J Nurs fles $2008.16(1) 75-85$

116. Lasene AW, Chidero A Cachat F, Paccad F, Bowet P. Ovenweight in Swiks children and associations with children's and parents' characteristics.

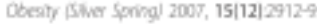

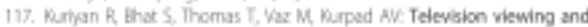
skep are associsted with overweight amoen urban and semivirban South indian children. Nur $/ 2007,625$

118. Lutfyya MW Lipshy MS. Wisdom-Behounek J, inparbutr-Marinkus M- is rural residency a tikk factor for owerweight and obesity for US dildwen?

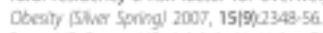

119. Butte NE, Puysu MR, Addolph AL, Vohra FA, Zaveri t Physical acthity in nonowerweight and overweight Hspanic children and adolescents. Med So Sports Ever 200?, 3969:1257-66

120. Wang Y, Ling H. Tussing L. Praunsthwein C. Cabalero B. Flar B-Obesity and velated risk tacters among low socioeconomic status minority students in Chicago. Puelic Hoath Mus 2007, 10197927.38

121. Tosthe AM, Ruclinger S, Borler E, Von KR. Adjusted population atributable fractions and preventable posential of risk factors for childhood obesity. Puble Heath Mut 2003, 1019:902-e.
122. Ortegs FQ, Tresaco B. Auie JR Moreno LA Martinthotilas M Meso IL Wamberg L Bueno M Tercedor P. Gudiemez A, Castilo ML Aveva Study Group Candioespinatory fitness and sedencary activities are associated with adiposity in adolescents. Obesily (siver Sachy) 2007, 15/61.1559.99.

123. Bellise F, Aollind-Cachera NF. Kelogg Sdentríc Adivisony Committee: Thee coneecutive (1993, 1995, 1997) surveys of food intake, nutritions attitudes and knowledge, and lfestyle in 1000 French children aged 9 11 years. I Hum Mutr Dier 2003,20031.241-51

124. Spinks AB Macpherson AK, Bain C. McOlure H.: Compliance with the Australian national physical activing guidelines for children. relationathip to ovenweight status. I Sol Med foort 2007, 1013) 156-63

125. Dedva I, OMaley PM, Johneton LD. Health-related behariors and overweight: a study of Latino adolescents in the United States of

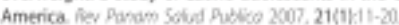

176 Ramos $\mathrm{E}$ Hamms H: Family and school determinams of everuxight in 13. year-old Pertugaew adolesconts. Acca Pandorr 2007, 96(21)281-6.

127. Liores S, Mahe B, wolatier Ih, Charles Mu Chid overweight in France and ts redationship with physical activity, sedentary behwow and sodoeconomic satus. Ex J On Nurr 2007, 61,4/509-10.

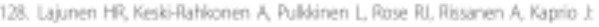
Are compuser and cel phone use wsociated with body maxs index and overweight? A population study among twin adolescems. aMC Pubcr thoob 2007,724

129. ve Velde SI, De B, Thorsdoetir L. Aasmussen M. Hagatromer M. Nepp KL, Brug $t$ Patterns in sedentary and exercise behaviors and assodiations with overweight in 9-14year-dd boys and girls-a cross-sectionsl study. auc Public hooloh 2007, 7:16

130. Bounele KN Hanan PJ, Neumar-Sztainer D. Himes JH bentification and correlanes of weight loxs in adolescemts in a national sample. Dessity

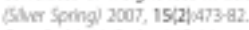

131. Canalod MM. Pader MC. Moreir PA Posado VM. Overweight and obesity

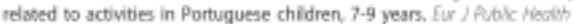
$2007,17(1) 42-6$.

132. Zakinkij MF, Noman GL. Salls JF, Caras KJ. Pawick K: Pattems of sedentary behawier amang adolekents. Heath Pychol 2007, 2611)113-20.

133. Handy $\amalg$, Dobbins T, Booth ML, noy-Whon E, Clisty, ND. Sedentary behaviours among Mustralian adolescents. Aust N Z S Pubic Heath 2006 . $3016) 534-40$.

134. Ekekund U. Frage S. Froberg K. Haro M. Andersen SA Sardinhs LB. Fidsuch C Andersen LF: TV viewing and phrysical activity ane indegendently ascocianed with mwtabolic risk in chidrent the Europer Youth Heart Study. F.05 Mar 2006, 3/1210488.

135. Moraes SA, Betran RU, Mondini L, Freitas IC [Prevalence of overweight and obesity, and associsted factors in school children from urban area in Chilpancinga, Guerrera, Merica, 2004: Cod Saude Publia 2006. 22160t $1209-301$, Portuguese

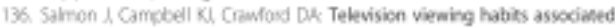
with obesing risk tacters: a survey of Melboume schookchildren. Med ) Cust 2006, 184(2) 54 . 7 .

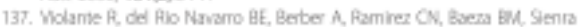
Monge It Obesity risk foctoes in the SAAC Onternational Study of Asthms and Alergies in Chidhoods in Mexico Oty. Aer Abyg Ner 2005. $5214)+41-5$

138. Rapp K Schick KH Bose H Weiland $5 K$. Type of kindergarten and other pocential determinants of overwoighn in preschool children. A.tes. Whooth Nur 2005, a61642.9.

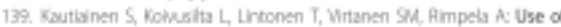
information and communication technology and prevalence of

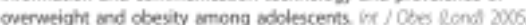
2916razs-33

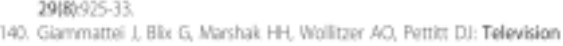
watching and soft drikk consumption associations with obesily in 11-

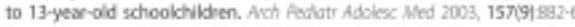

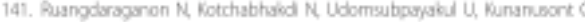
Surigwoncosisd $P$. The association between television viewing and childhood obesity a national survey in Thaland. I Mod Awoc Tha 2002 8S|5uppl 4) $51075-80$

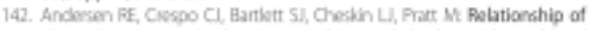
physical activity and television watching with body weight and level of fatness among children results from the Third National Health and Nutrition Examingtion Survey. HuA 1996, 279/121938-42. 
143. Gortmaker SL. Must A Sobol AM Peterson K Coldetz GA, Dieter WH Television vipwing as a cause of increxsing obesihy among ctilden in the Uvited Stater, 1986-1990. Anch Prylat Adaksc Med 190 150|4/356-62.

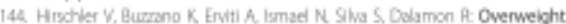
and lifestyle betsribrs of bow socioeconomic elementary school thildren in Buenos Aires. BuC Pactotric 2009 , 9.11.

145. Tsai H-L. Tsai HC. The association of BMS and sedenary time with espiratory symphoms and asthma in 5 th gade shookchidren is

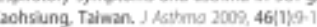

146. Trang NHHO, Hong TK, Dbley ML, Sibbrit DW. Factors associsted with physical hactivity in adolescents in Ho ON Minh Cry, Metnam. Med So soots Evec $2009,4117 \times 1374.81$

147. Alus SA, Lasson K. Franke TM Hollon NE Associations between media use and health in U5 cilden, Acod Pedian 2009, 955:300-6

148. Nawal LM, Garcla R., Darkwa CM, Young T, Lipkby MS. Owewwight and obese prevalence rates in African Mmerican and Hispanik children: An

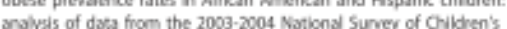

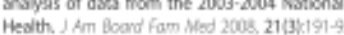

149. Colins AE, Pakic \& Pack CL: Factors sossocisted with obesity in Indonesion

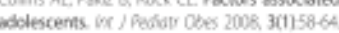

150. Gomez LF, Para DC, Lobelo F, Samper B. Misene l. bocoby E, Lucumi D Mroudo S, Bouda C. Tetevikion viowing and its association with overweight in Colombian childent: Pesults from the 2005 National

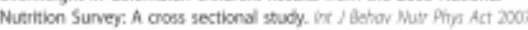
4/411.

151. Batiow SE Chang fft is parentel aggavation associated with childhood ovenweighte An analysis of the national survey of chilbren's bealth 2003

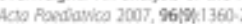

152 Uner 1 Scrago R Schasf D, Fitagerald E. Wilson $N$ Comelates of body mass index among a nationally representasove sample of New Zealand index among a nasionaly representaove sarpit

153. Tsai H-l. Tsai AC, Nrisgu I, Ghosh D, Gong M. Sondretto k Associstione of BM, TV-watching time, and ptrsical activity on resciratory symeceoms

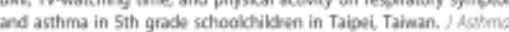
$2000,4451397-40 \%$

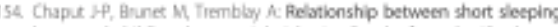
hours and dilikhood overweightlobesty. Reylts from the Quebec of

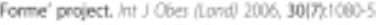

155. Andersen LF, Lilegasid IT, Overty N. Lyle L. Kleps K-t Jchanssan L Dvenweight and obesity among Nonwegin schookhiddrer Changes from 1993 to 2000 s scand S Public Heath $2005,3312299-106$

156. Ghron S, Lambert 1, Neate D. Associations between weight status. physical activity, and consumption of bisouts, cakes and confectionen

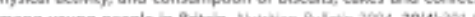

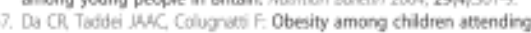
chementar public shook in Sao Pada. Brazi: A case-centrol study.

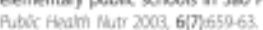

158. Taylor WC, Salis J, Dowda M, Freedson PS, Eacon K, Pate Ff: Activty pasterns and comelates among youth: Differences by weight status. Peatay Ever So 2002, 14/4/2415-3).

159. van Zutphen M. Bell AC Koemer Pl, Suritbuen BA. Association between the Family erwircmemt and television viewning in Austrdian children. Poedion Chid Healh 2007, 43061 458-635

160. Lapiou A Para M Correlates of childhood obosiny in Athens. Grevce.

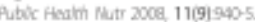

161. Smith Bu, Phongasan P. Haves D, Halavarau Y, Ohey T: Body mass indec, physical activity and detary behsviours amom adolescents in the

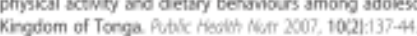

162 Shields Mt Orerweight and obesify among dilben and youth. Heoly

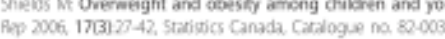

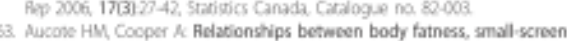

sedentary activity and regionalty among schoodchildren in Victoria. Sedentary activity and regionality among schod

6A. Boone JE, Gondon-Larsen P, Adder LS, Popken BU. Screen time and physical

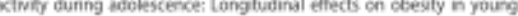

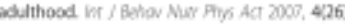

165. Wein Fluat C, Ouja M. Wagrer A, Haan MC, Arveler D, Scollenger l. Imon C. Physical acthity is imversely related to waikt cicumberence n

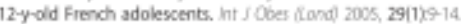

66. Jenssen L, Katzmascak PT, Boyce WE, Kng Mh, Pickett W: Overweight and obesicy in Canadion adolescents and their associations with dietary

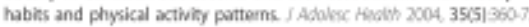

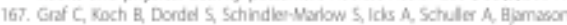
Wetrens B, Toharsik W, Predel HG. Physical activity, letsure habits and

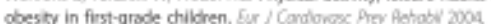
1116208490.

168. Stevler N. Signer TM Suner PMt Electronic games and envirenmental factors associand with childhoed obesity in Switzeland ches pes 2004 $1216) 906903$

169. Parnick K, Norman Gil, Calfas KL, Salls JF, Zabinaki MF. Diet, physical acthity, and sedentary behawors as risk factors for ovenweight in adolescence.

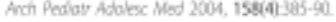

170. Vindewder EA Shim M5, Coplovitz AGC Linking obesity and activity level

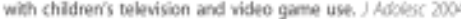
2011271-89

171. Utter 1 Neumak-Stainer D. Jeffery R. Story Mt Couch potatses $\alpha$ french fries: are sedentary behwioes associsted with body mass index, phrsical

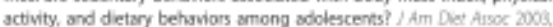
10ख10

172. Trembly MS, Wilms ID. 6 s the Canadian dilahood obesity epidemi

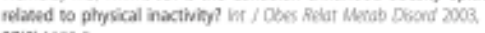
$27 \mid 9 k 11005$

173. Lowy R, Wechiser H, Galusia D, Futon E, Karn L- Television viewing and its associations with overweight, sedentary lifestyle, and irsufficient consumption of fruts and vegetables among US high school students.

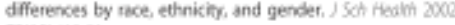
$72|10|<|3-2|$

174. Janz WF, Lew S SU, Bums Th, Torner XC. Wring MC, Wamen II Fatness, physical activity, and tedevision viewing in children during the adiposity rebound period: the bowa Bone Development Studf. Per Med 2000, $3516665-71$.

175. Esenmam IC, Bartee HT. Wong MC. Physical astivity, TV viewing and weight in US. youth; 1999 Youth Risk Behavior Survey, Obes fes 2002 1015) 379.35 .

176. Goudon Larsen P, Mdair LS, Poptin BM. Ethnic differences in physical activity and inactovity pasterns and overweight status. Che fRe 2000 , $10 \mid 3 \times 141-4)$

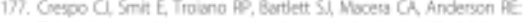
Tekerision wakting, energy intake, and obesity in US chïdretr: results from the thied National Health and Nutritien Ecamination 5urvey, 198:

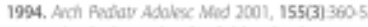

178. Hemsndez B. Gortmither SL, Coldtz Gh Peterson KE, Laid MM PanzCatrera S Associstion of obesity with physical actwity, telerision progarms and other forms of video viewing among children in Mexion

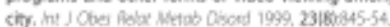

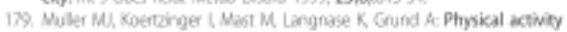

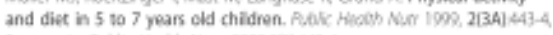
Ematum in: Public Heath Nitr. 2000,317/443/4

190. Obarzanek E, Streber GB, Craword PB, Goldman SR, Bamier PM Frederick MM, Lakstos E. Energy intake and physical activity in relation to inderes of body fat the National Heart lung and siood institute Growth and Heath Study. Am I Oin Mus 1994, 6011 K15-22.

181, Dietz WH Jr, Gonmiber Sl: Do we tateen cur childen at the belevision sot? Obesity and tolevision vivwing in children and abolescemes Pedionics 1985, 75(5) $1807-12$

182. da Stwo RC, Malina AM: Ileved of physical activity in adolescents from Niteroi Alo de laneiro, Bravil. Cood Soude Putuca 2000, 1614:1091-?. Portuguese

183. Asonte PM C Cos 1 Sorrevile K Samver AC. Tiveras EMt Overweight prewention in pediatric peimacy care: a nevds assexsment $d$ an urtan

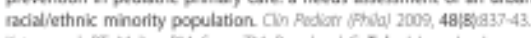

184. Katzmatzk PT, Maina FM, Song TM, Bouchard C. Television wiewing physical activity, and health-related fitness of youth in the Queber Farmily Study. I Adolec hooth 1998, 25/5/318-25.

185. Puath \. Longinger T, Ragheb C Candax risk eviluation for elementary

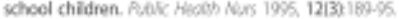

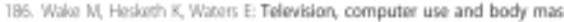

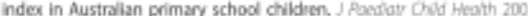
$39 \mid 2 \times 130-4$ 


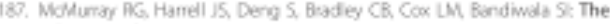
infuence of physical activity, socioeconomic statw, and ethnicity on the weight status of adolescents. Obes fes 2000, B/21:130.9

188. Forshee PA, Andersan PA, Stoney ML: Associations of various family characteristics and time uke with children's body mass inder. Communty Heath Mus 2009, 2602137-86.

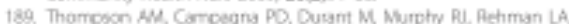
Wadswonth SA. Are overweight studemts in Gades 3, 3, and 11 less phrsicaly active than their healthy weight counterparts? ho J Pedian apes $3009,40728.35$

190. Hume C, Singh A, Bug J, Mechelen W, Chinapsw M. Dose-response associstions between screen time and overweight among youth. hit Pediat Obes 2009, 4(1)661-4

191. Alves JG. Siquesro PP. Figuesios IN: Overweight and physical inactivity in children living in faredas in the metropolitan negion of Recife, Brabl. $f$

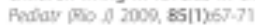

192. N SH Veseedenen C, Nbdeen Z, Cans E. Mass L: Associations of overweight and of weight dissatisfaction amang Palestinian adolescents: fnding: from the national study of Palestinian schookchildren $f$, hum fiutr Dief $2008,2211) 40-9$.

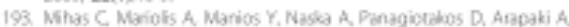

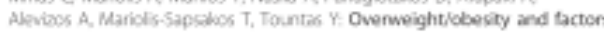
associated with body maxs index during adolescence the VYFONAS study. Acta Ploedion 2009, 98/30495-900

94. Mikdzicayk RT, Aictiver M: Associations of behavioural, psychosodial and socioeconomic factors with over- and underweight smong German

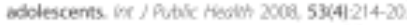

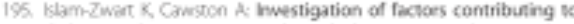
diabeess risk in American Indian/Alaska native youth. Am indas Ams

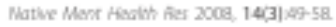

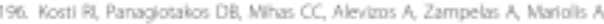
Tourtas Y: Oietary habiss, physical sctivity and prevalence of overweightiobesity among sdolescents in Greece: the Wyrongs study. Werweghtidbesty among sodolescents

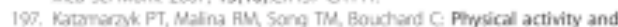

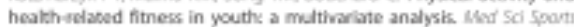
Ever 1996, 3015)709-14

196. Wasghan C, KIleinen A, Heisaso S, Lastikinen T, Dunbar $t$ The dietary, physical activity and sedentary beheviours of Victorian regions secondary school students. Aus $J$ Prim Hooks 2007, 13/1181-6.

199. Moca A, Rateino it sancos MP, Gomes H Obexity, phryikal activity, computer use, and TV viewing in Portugesew adolescents. Padiorr Eom Scl 2006, 18:17:113-21.

200. Colwed A, Kato M: Imestigation of the relationship between social isolation, sef-esteem, agoression and compuner game play in lapanese adolescents. Aion Jound' of Socol Pyychology 2003. 6(2) Aug -158

201. Fionshe AA, Anderson PA 5torey ML. The role of beverage consamption physical actovity, sedentary bohavior, and domagraphics on body mass physical activity, sedentary bohavior, and somagraphics on b

202. Waler CE, Du S, Poplin EM. Paterns of overweight, inactivity, and snacking in Chinese children. Obes Afer 2003, 11/39957-6

208. Wolf AM, Gortmaker SL. Cheung L, Gray HM Heroog D8, Coldtz Gk Activity, inactivity, and obesing raciel, ethnic, and age dfferences among schoolgive, Am / Pubcíc Healh 1993, 8301 1)1625.

204. Fobinson TM, Hammor D, KIlen JD, Wrasmor HC, Wiloon DM, Hawand C, Taylor CE. Does television viewing increase obesty and neduce physical activity? Cross-sectional and longtudinal analyses among adolescent givts. Pedianis 1993, 91(2)273-90.

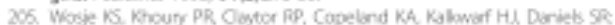
Adiposity and TV viewing ice nelated to less bone accrual in young

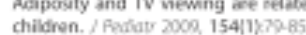

206. Tucker LA: The edationship of television viewing to physical fitness and obesity. Adolescence 1936, 21 (BS4) T97-BDA.

207. Allarwari S, AHHashmi K, Ah-Abri M, lviu D, Hersan Mo EHects of overweight and leisure-time activities on aerobic fitness in urton and rural adolessents. Metab 2009, $714 ; 369$-74

206. Lobelo F, Dowda M. Pfeitter Ka. Pate Fift Bectronik mecia exposuee and its arsociation with activity-relased cutcomes in tomale adolesconts: crors-sectional and longtudinal andiyser. 5 Phr Act Heath 2004 6:2:137-43
209. Hardy U. Dobbins TA ney-Wikson EA Chody AO. Bosth ML: Sedentainess, umal-screen recreation, and fitness in youth. Am $f$ Prev Med 2000 $36 \mid 2 \times 130.5$

210. Pate HR, Wang CY, Dowda M, Famell SW, ONeil IR Candiorespiratory frness levels among US youth 12 to 19 years of age: findings from the 1999-2002 National Heath and Nutrition Examination Survey. Anch 1999-2002 National Heath and Nutrition tor

211. Dolman f, pidey K. Ditterences in body Gatness, fas patserning and carbo-respiranory fitness between groups of Australian children formed on the bass of physical activity and television viewing guidelines. $f$ Plys Act Healih 2006, 3121191-199

212. Tudber LA: Television, teenagers, and health. I) routh Adalexc 198: 1615 ) $415-25$

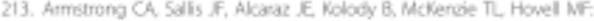
Children's television viewing, body fat, and perysical foress. Am / Healon. Promot 1978, 12|65)3638.

214. Tudber LA Hage FL, Tucker LA, Hager AL: Televikion viewing and

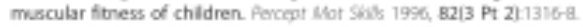

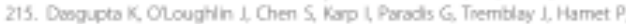
Piote L-Emergence of sex alferences in prevslence of high systolic

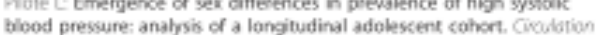
2006 pressure: anatrse d a langtudinal adolestent cahort.

216. Dergapta K. Sex ditlerences in the development $\alpha$ higher systolic blood pressure during adolescence. Contialagy ficwiew 2008, 25|5) 54 -3

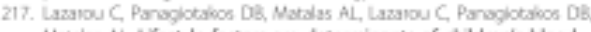
Matalas AL: Lifestyle factors are determinants of children's blood

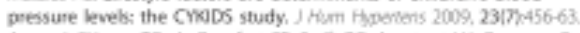

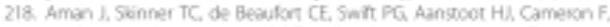
Hividoene Soudy Group on Chilhood Dubetes. Associations botwoen physical activity, sedentary beharioe, and ghycemic control in a large cohort of adolescents with type 1 dabetes: the Huldoere Study Group on Childhood Oisbeves. Podist Diaberes 2009, 10162024.9.

219. Overty NC. Mongeirsdottir HO. Brurbong C. Andossen SA Andersen LF. W. Jangensen K. Norwegian Study Group for Chadhood Dabetes. Pfysical activity and owerweight in childen and adolescems using imensifed insuin trearment. Pediay Dshases $2009,10021135.41$.

220. Mark AE, lansen $t$ Aelationship between screen time and metabolk

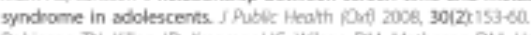

221. Aobinson TN KIen JD, Fraemer HC, Wison DM, Matheson CM, Horkel M.

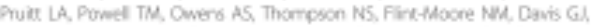
Emig Kh Brown RT, Fochon L. Geen S. Vacady A: Dance and reducing todevikian viewing to prewent woighe gain in African Amwerican girts the Standond GEMS pilot study. Ethn Dis 2003, 13/5uppl 1/S65-73.

222. Holder MD, Colemen B, Sehn Z., Hoider MO, Coleman B, Setn 2L: The conterbution of active and passhe kisure to children's well-being.

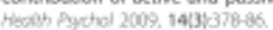

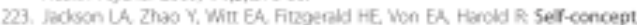
seltesteem, gender, race, and information technology use. Chemerchel Schor 2009 , 12441437 .

224. Neumark-Sztainet D, Goeden C, Story M, Wal M: Associations between body satisfaction and physical activity in adolescents: Implications for programs simed at preventing s beosd spectrum of weight-related doonders fating Dosondses 2004 1202:125-3?

225. Dominick JAR Viseogames, televikion violence, and aggession in toenagers.

225. Ohannessian CM: Meda use and adolescent psychological adjustment: An examination of gender differences. J Chat fam Stud 2009 $1815563-91$

227. Wigins JD. Sel-esteem, earned grades, and television viewing habits of students. Sohool Counsebor 1967, 35:21:128-133.

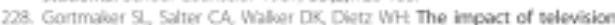
viewing on mental aptitude and achievement: A longitudind study.

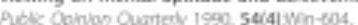

229. Mitry KB, Mrhovitz CS, Strobino DM, Boradoowse CL: Onilden's television exposure and behsviosal and social outcomes at 55 years does timing

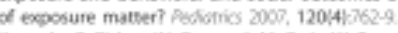

230. Kunsche E Fichert W, Drerpeck M Caig W, Boyce W, de Matos MG Tecovision viewing and forms of bullying amang adolescents from eight countres. 


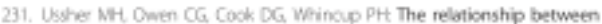
etryikal activety, sedentary behaviour and psychabgical wellbeing

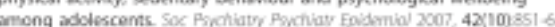

232. Chowhan 1 Scenart JMe Television and the behaviour of adolescents! does socio-conomic atatus moderate the Inkt Soc Scl Mlod 2007 ? 65: $1324-36$

233. Nebon MC, Gordon-Larsen P. Ptyrical activity and sedentary behavior patterrs are associated with selected adoleckent health risk behavions.

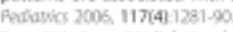

234. Page AM, Zarco EP. Pelationchip besween television vieweing frequency and scores on stryness among Philippine high school students. Pyyche Alep 2001, 89(2) 305-8.

235. Bnodersen NH. Steptoe A, Wilamson S, Warde I: Sociodemographic, developmental, environmental and pischological conrelotes of ptysicd activiny and sedencary behwicr at age 11 to 12 . Ans Behor Med acos. 280112:11,

236. Salamoto A: Mdeo game use and the development of sociocognitive belities in childern: Three surveys of elementary school students. A A Soc Pyychol 1994, 2411,21-4).

237. Ennemose $M$ Sctreeder $W$. Aelations of telerision viewing and reading: Findings from a 4year lenghuesinal study. I Eoluc Pychor 2007 . 902130969

238. Lonner Wh, Thomd he FU, Forbes NE, Astrwarth C. The influence of television on measured coconitive abilities: A study with Nathe Alaskan children. $/$ Coss Cut Ayychol 19es, 16/3/35s-80

239. Shin N: Eaploring pottruays from television viewing to academic

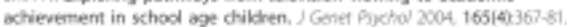

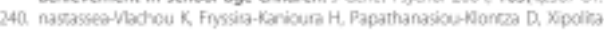
Zachariad A Marsaniotis N. The effects of television verwing in Grevce. and the role of the paediatrician: a familar tolangle revisited. Aus Pexlay 1996, 158/12t:1057-60.

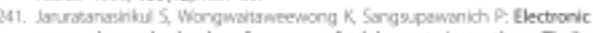
game play and schood performance of adolescents in scutthem Thailend. Getepsochol betov z009, 1215)509-12

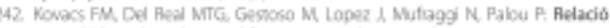
entre habits de vida i qualficacions escolars en adolescents. Apunts Mextcha de /Esport 2008, 43|160k 181-8, Sparish.

243. Wistionsson AL. Sigfuedontr IQ, Alecrante. P. Helowon AR Adolescent hesth behavicr, contentment in school and academic achievemer. An 1 Heowh Betwor 2000.33 (1)69-70

244 Shait ( Sament JD. Asscoiation between velevision, morie, and video game exposure and school pertormance. Fentawics 2005, 11844) 1061-70

245. Caldre SI, Baniation CI Black and white TV: Race, television vieaing and academic achievement. Sociobogcor Specrum 1999, 19/1139.

246. Sheival \&. Television viewing of ligher secondary students: Does i: atfect their academic achievement and mothematical reasoning? Pyctwol Dev $50 \mathrm{C} / 2006,18221201-13$

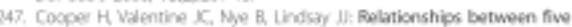

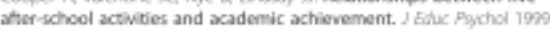

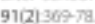

248. Welch WW, Walberg Hl, Fayser Bl: Predicting elementary science leaming using notional assessment dats. foumb of Aeveanch in Soance Teaching 1966, $23060699-704$

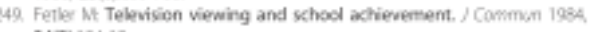
34(2) $104-18$

250. Fobers DF, Bachen CU, Homby MC, Hemandezfamos P: Reading and ielevision: Predictors of resding schievememt at different age levels. Communionon Peseanch 1934, 1111) 949 .

151. Waberg HL, Tsaist Aesaing schievement and dimivishing retums to

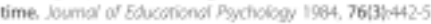

252. Fidley-bohron R Cooper H, Chance I) The melation of childen's velevikian

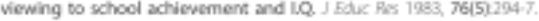

253. Walberg HA, Weinstein T: The production of achievement and attinude in

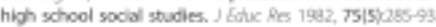

254. Scott UF: Aelationships between elementary school chïdren and television. / Eorke Res 1958 52134-7.

255. Dumaks S4 Cohart and gender diflerences in extracumicular participation: The retriconship between activitios, math achiewement, and college expectations, Socid finectr 2009, 2911/g2.

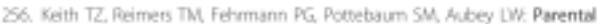

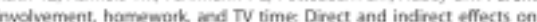
high school achiwwment. I Edw Piychy 1965, 78:5/373.80

ZST. Gaddy GD: Teterikion's impact on high school achierement. Public Dain Q 1996, 50/31340-59.

25s. Skoric MM, Teo $L$, Neo PL: Children and video games addiction engegement, and scholastic schievement. Cjochygchal behor 2009 , $1215) 567-72$.

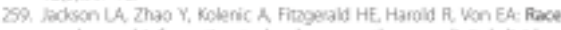
gonder, and infomation wechnology uxe: the new digral divide.

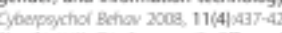

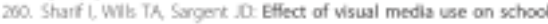
performance: A prospective study. I Adolesc hibath 2010, 46:1182.6\%

261. van Schie EG. Children and videogames: Lesure activities, aggression. socied imtegation and school performance. $f$ Acol SOC Byochor 1999. $27|13|: 1175.94$

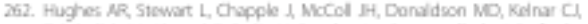
Cabihollsh $M$. Ahmed F. Felly I. Randomized, controlled trial of a best practice ind vidualimed behavional program for trestment of chid hood cyerweight: Scottish Childhood Orermeight Trestment Trial (SCOTH.

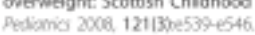

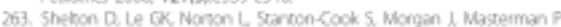
Randomized conoolled triat: A parent tarsed group education progam for ovenweight children J Plantaty Ohad Heath 2007, 431121/799905.

204. Goldfield GS. Malory R, Parber T, Cunningham T, Logg C Lumb A, Parker K, Prud homme Q. Adsmo 12: Effects of open-loop feedback on physical activity and selevision viewing in overweight and obese clilden:

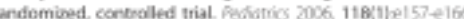

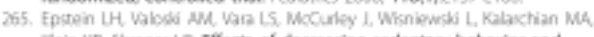
dein KR, Shiager LR Effects of decreasing sedentary behavior and increasing actovity on weight change in obese children. Heolth Pyycha $1995,14[2 ; 109-15$

206. Trembley MS, Leflenc AG, Lanssen L Who ME, Hids A, Munumets K: Canadian sedentary behaviour guidelnes for chädren and yoush Apr. Fiysiol Narr Meaco 2011, 36(1)59-64

2ij. Kho ME, Eva KW, Cooi DI, Brouwers OC. The Complewness of Feporting (COFE) index idenofies important deficiencies in observational study conference abstracts. I On Epitemiol 2008, 61112:1241-s

208. Hopewell S. Clarke M. Ackie L: Aeporting of trials presented in conference

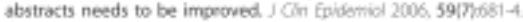

269. Leflinc AG lanssen t Defference between serf-reported and acceleremecer mexured moderme-sovigorous physical activing in youth. Aadotr Exer So 2010, 22 223:34.

270. Adamo KB, Prince Sh, Tricco AC, Connor-Gorber S, Tremblay MS. A comoarison of indibect versus direct measures for assessing physical activity in the pediatric population A systematic review. At I Pediat? Obes $2009,411 \times 2-27$ ?

271. Pddsks $\mathrm{E}$, hwin \& Adtive video games to promoe physical activity in

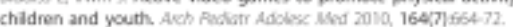

\section{soi:10.1186/1479-5968-8-89}

One this article as Tremblay at al: Systemasic review of sedentary

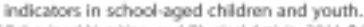

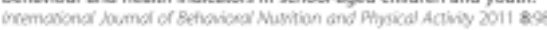

\section{Submit your next manuscript to BioMed Central and take full advantage of:}

- Convenient online submission

- Thorough peer review

- No space constraints or color tigure charges

- Immediate publication en acceptance

- Inclurion in Pubneed, CAS, Scopus and Google Scholar

- Research which is freely available for redistribution

Submit your manuscript at

( ) BioMied Central 


\title{
Video Game Playing Is Independently Associated with Blood Pressure and Lipids in Overweight and Obese Adolescents
}

\author{
Gary S. Goldfield ${ }^{1,2,3,4,5 *}$, Glen P. Kenny ${ }^{2}$, Stasia Hadjiyannakis', Penny Phillips ${ }^{6}$, Angela S. Alberga ${ }^{2}$, \\ Travis J. Saunders ${ }^{1,2}$, Mark S. Tremblay ${ }^{1,2,3}$, Janine Malcolm ${ }^{6}$, Denis Prud'homme ${ }^{2}$, Rejeanne Gougeon ${ }^{7}$, \\ Ronald J. Sigal ${ }^{8}$ \\ 1 Healthy Active Living and Obesty Feseanch Group. Children's Horpital of Earsem Oncario Fesearch Insthune, Otawa, Ontario, Canada, 2 school of Human Kinetics, \\ Faculty of Hesth Sciences, Universicy of Ottawa, Ottawa, Onearia, Canoda, 3Department of Pediacics, University of Ottama, Ottawa, Ontario, Canada, 4 School of

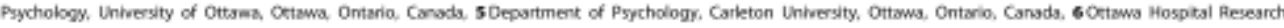

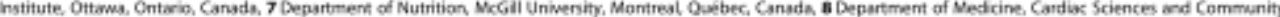 \\ Health Sciencer, Faculies of Medicine and Kinesiology, University of Calgary, Calgary, Alberta, Canadia
}

\begin{abstract}
Objective: To examine the assoclation between duration and type of screen time (TV, video games, computer time) and blood pressure (BP) and lipids in owerweight and obese adolescents.

Design: This is a cross-sectional study of 282 overweight or obese adolescents aged 14-18 years ( 86 males, 196 females) assessed at baseline prior to beginning a lifestyle intervention study for weight control. Sedentary behaviours, defined as hours per day spent watching TV, playing video games, recreational computer use and total screen time were measured by self-report. We examined the associations between sedentary behaviours and $\mathrm{BP}$ and lipids using multiple linear regression.

Results: Seated video gaming was the only sedentary behaviour associated with elevated BP and Fids before and after adjustment for age, sex, pubertal stage, parental education, body mass index (BMI), caloric intake, percent intake in dietary fat, physical activity (PA) duration, and PA intensity. Specifically, video gaming remained positively associated with systolic $B P$ (ad)usted $r=0.13, \beta=1.1, p<0.05$ ) and total cholesterol $/ \mathrm{HOL}$ ratio (adjusted $r=0.12, \beta=0.14, p<0.05$ ).

Conclusions: Playing video games was the only form of sedentary behaviour that was independently associated with increased BP and lipids. Our findings provide support for reducing time spent playing seated video games as a possible means to promote health and prevent the incidence of cardiovascular disease (CVD) risk factors in this high risk group of overweight and obese adolescents. Future research is needed to first replicate these findings and subsequently aim to elucidate the mechanisms linking seated video gaming and elevated BP and lipids in this high risk population.

Trial Registration: Clinicaltrials,gov NCT00195858

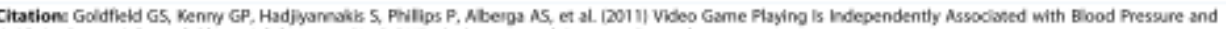

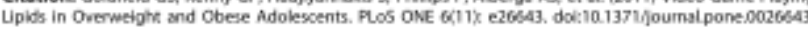

Editer: Phlipge Foust, I2MC INSERM UMR U1048, France

Received Augua 17, 2011; Accepted September 30, 2011; Published Nowember 1, 2011

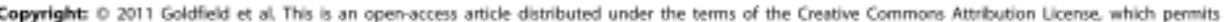
unrestricted use, distribution and reproduction in any mediam, provided the original suthor and source are coedited.

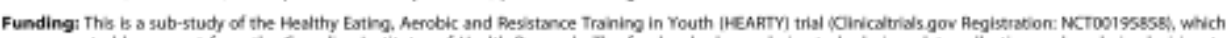
was supported by a grant from the Canadian hatitutes of Health Resesrch. The funders had no role in study design dats collection and ansibysis, decibion to pubish or preparation of the manuscript GSG is the CHEO Volunteer Association Endowed Scholar. GPK is supported by a Uriversity of Ottawa Alesearch Ohar. (a)

Competing Interests: The outhors hewe declared that no competing imterests exibt.

- E-mat:
\end{abstract}

\section{Introduction}

Most adolesoents living in Western countries spend excessive amounts of time being sodentary, mainly in the form of screen time behaviours such as TV vicosing, sated video gaming, and recreational computer use [1-3]. This is concerning becaluse sedentary behaviours track throughout adokesenoe and into adulthood [4], and sedentariness via screen time in adulabood is associated with increased morbiclity and mortality, making the relationship between sedentary behaviour and health indicators an

Q. PLOS ONE I wwwplosone.org important area of study [5], Many of the studies conducted in the pecliatric population bave focused primarily on glucose-relatex measures with relatively little attention to traditional cardiovascular disease (CVD) riak factors such as high blood pressure (BP) and dyslipidemia. Howener, there is evidence that dyslipidemia and elevated $\mathrm{BP}$ are being increasingly observed in youth, especially overweight and obese youth $[6,7]$, and that these CVD risk factor in adolescence prectict the developenent of CVD and mortality in adulthood $[8,9]$. Moreover, animal research has shown that extended bouts of sedentary behaviour result in dramatic 
reductions in lipoprotein lipase activity leading to reductions in high-density lipoprotein cholesterol (HDL-C) in rat skeletal musch [10]. Similarly, escessive sedentary behasiour is associated with increases in low density lipoprotein cholesterol (LDL-C) and riglycerides in adult humans [11].

In the studies focusing on $\mathrm{BP}$ and lipids as an indicator of CVD risk in youth, screen time was independently associated with increased CVD risk after adjusting for moxterate-tovigorous intensity physical activity $(\mathrm{PA})$ and other confounders in some studies [12-14] but not others [15-17]. However, the studies demonstrating null findings have design limitations including limited statistical power [16], TV viewing as the only index of sedentary behaviour [15], a limited number of measured CVD risk factors [15], and the assessment of screen time not including video gaming [17]. Including seated video gaming as part of screen time measurement is important because it is appealing to adolescents [18] and is associated with obesity in youth [19-21], possibly due to increased food intake $[22,23]$. Moreover, recent research found that different types of acreen bebaviour may bave different and independent effects of chronic disease risk in wouth [24.25] but again these studies failed to include video gaming as part of screen time. Thus, measurement of time spent playing video games in association with the full spectrum of $\mathrm{BP}$ and lipid measurements as indicators of CVD risk factors remains unclear

The parpose of this study was to examine the independent relationshipe between the volume and types of sedentary sereen time behaviours (i.e., TV viewing, seated video games, computes time) with systolic and diastolic BP and lipids in overweight and obese adolescents, controlling for a wide range of confounders.

\section{Materials and Methods}

\section{Participants}

The sample consisted of 282 adolescents who were either obese [ 295 body mass index (BMI) percentile for age and sex) of werweight $\left(85^{\text {th }}\right.$ to $94^{\text {th }}$ BMII percentile for age and sex) based on cut-off values from the Centers for Disease Control (CDC) sroweth charts [26], recruited as potential participants in a diet and exercise trial. Those participants who were ovenveight had to have at least one comorbid CVD or diabetes riak factor, including family history, to be induded in the study. The sample was comprised of 86 males and 196 female aged 14 to 18 years, with mean age of $15.5 \pm 1.4$ years. All participants were Tanner stage IV or $V$ with respect to pubertal development, and the mean BMI was $34.4 \pm 4.3 \mathrm{~kg} / \mathrm{m}^{2}$. The majority (70\%) of the sample was Casocasian, $12 \%$ were African Canadian, $2 \%$ Asian, $3 \%$ Hispanic. $2 \%$ First Nations, $4 \%$ Arabic, $4 \%$ mixed race, and $3 \%$ categorized as other. Parens of participants were well edacated, with $75 \%$ of mothers and $68 \%$ of fathers having completed some university of community college program. Participants were recruited by posters and advertigements in the Children's Hospital of Easten Ontario's endocrine/obesity clinic, by radio and bus advertise ments, and flyers in communiry physicians' offices. Bus advertisements were the most effective recruitment strategy, accounting for 485 of recruitment. Data for this analyxis come from the Healthy Fating and Acrobic and Resistance Training in Youth (HEARTV) trial, a randomized controlled exercise intervention aimed at redacing adiposity in obese adolescents. The data reported berein represent baseline data collocted before the intervention from 2005 to 2010 . Of the 3.58 adolescents, 282 (79\%) had complete data for analysis from the baseline data collection of the HEART) trial. All participants completed the testing individually (i.e. one on onel with the resareh coordinatos.

\section{Ethics Statement}

Informed written consent from each participant was obtained in accordance with the principles expressed in the Declaration of Helsinki guidelines for human subjects. In the case of minors, written informed consent was also obtained by parents or legal guardians. This study was reviewed and approwed by the Research Fthics Boards of the Children's Hospital of Eastern Ontario (CHEO) and The Ottawa Hospital.

\section{Screen Time Behaviour and Physical Activity}

Time (hours per day) spent watching TV, playing seated (inacrive) video games (excluding compoter games), and recreational computer time (excluding school work) was assessed by selfreport questionnaire. Total screen time was ealculated by agsregating the three types of screen hehaviours. Self-reported PA duration was cakculated based on the question "On average, how long do you participate in some sort of physical activity each day - with physical activity being cumulatise not conscutive." Likert-type rating seales included 6 response ratings, whereby $I=k \times s$ than 5 minutes, $2=5$ to 15 minutes, $3=15$ to 30 minutes, $4=30$ to 45 minutes, $5=45$ to 60 minutes, and $6=$ greater than 60 minutes. Intensity of $\mathrm{PA}$ was asessed by the question "on average, bow would you describe the intensity of most of your physical activity?" Response ratings included I = light, $2=$ mod. erate, and $\mathrm{S}=$ vigorous. Both $\mathrm{PA}$ duration and $\mathrm{PA}$ intensity were used as covariates in our statistical analyses.

\section{Lipids}

Onernight-fasting blood samples of aporoximatcly $20 \mathrm{~mL}$ of venous blood were taken in the morning, from a forcarm or antecubital vein and were transported directly to the laboratory at the Ottawa Hospital for anahsis. The lipid profile measurements included triglycerides (mmol/L), tocal cholesterol $(\mathrm{mmol} / \mathrm{T}$, and HDL-C (mmol/L) which were measured by using encymatic methods on a Beckman-Coulter LX20 analyzer (Beckman instruments, Brea, California), while L.DL - C concentrations were calculated by using the Friedewald equation [27]. Total cholesterol/HDL-C ratio was derived from measured values.

\section{Blood Pressure}

BP was measured manually using a mercury sphymmomanom. eter on the left arm after 4 minutes of rest, with subjects sitting with their back supported. Three BP measurements were taken at 1-minute intervals the mean of the final two measures of BP was used for the anahsis.

\section{Covariates}

Weight and height were measured using a Health $\mathrm{O}$ Meter manual scale (Health O Meter, Continental Srake Corp, Bridgevixw, II.L. while participants wore light cloching, without shoes, using standard techniques. BMII was calculated as weight (kg)/ height in metres ${ }^{2}$. Children were classified as overweight or obese according to age and sex specific cut-points as dexribed above in the participants section [26]. Sexual maturity was assessed using the 5 stage seale for breast development in females and testicular volume in makes, acoording to Tanner [28].

Participants completed questionnaires on the same date as the physical measurements were taken. They were asked to report their age, sex, race and whetber their mother and father completed elementary school, high school, communiry collkge or university. Highest level of maternal and paternal education was assessed as a proxy for soxio-cconomic status.
November 2011 | Wolume 6 | Issue 11 | e26643 
Participant dietary intake, induding total kilocalories consumed. kilocalories (and percent kilocalories) from fat, protein, and earbohydrates were assessed using 3-day, 24 hour food diarie under the supervision of a registered dietitian. The research coordinatoe provided instruction on food recoeding prior to evaluation of energy intake, and participants were provided with tools to aid in measurement. Food records were completed for three days, two weekdays and one weekend day, and the meat intake was averaged across the three days using food composition analysis sofiware The Food processoe SOL. 2006, ESH Research, Salem, OR). A registered dictitian met with each participant shortly after completing the 3-day food records to clarify recording and gain more accurate measurement of energy intake.

\section{Statistical Analyses}

The distributions for $\mathrm{BP}$ and lipid outcomes were examined to determine if they were normally distributed. All distributions were normally distributed except for trighyorides, which was positinely skewed but normalized when it was logarithmically transformed, and this transformed triglycerides variable was used in the analyses. Sex differences were examined by independent t-tests and chi-souares for continuoss and categorical variables, rexpectively (Table 1). The relationships between the covariates and the individual BP and lipid outcomes were assesed using Pearson Correlations for continuous variables and Spearman Rho fnooparametric) correlations for categorical variables (Table 2). The independent associations berween the types of sedentary behaviour (seated video games, TV, recreational computer time, total sereen time) and PA duration and PA intensity with $\mathrm{BP}$ and lipic measures (dependent variables) were tested by multiple linear regression, adjusting for sex, age, highest level of maternal and paternal education, sexual maturity, BMI, total cakoric intake, percent of calories from dietary fat, PA intensity and PA duration Table 3) Each regression foe the askociations between sedentary behaviours and BP and lipid outcomes were aloo asoesocd for sex Xsedentary hehaviour interaction. Regarding the association berween PA duration and PA intensity and BP and lipid outcomes, total screen time was used as the sedentary behaviour in a regressions because it represents an aggregate measure of the time spent in all sereen time behaviour. For regressions involving PA duration and $\mathrm{PA}$ intensity, sex $\times \mathrm{PA}$ interactions were also asoessed for each BP and lipid outcome. Seatistixs were analyzed with SPSS for Windows (Version 18) and a p-value $=0.06$ denoted statistical significance.

\section{Results}

The descriptive characteristics of the sample are shown in Table 1. On average, makes bad a higher BMI, reported more time spent playing seated video games, greater total screen time, and greater caloric intake compared to females. Males were significantly more likely to be obese than females $2100 \%$ vs $88 \%$. p<0.001). Males alko had higher systolic BP, triglycerides, and total cholesterol/HDL tatio, while females were more sexually mature and had higher HDL-C. There were no sex differences on self-reported PA.

Unadjusted correlations are shown in Table 2. None of the sedentary behaviours was significandy correlated with BP or lipid. exoept for time spent playing video games, which was poesinely associated with systolic BP $\{r=0.20, p<0.001\}$ triglyceride: $\langle r=0.10, p<0.001\rangle$ total cholesterol/HDL $-\mathrm{C}$ ratio $\langle\mathrm{r}=0.19$ $\mathrm{p}<0.001)$, and pexatively associated with HDL-C ( $\mathrm{r}=-0.19$ p $<0,001)$, Although PA duration was bot aseociated with any BP
Table 1. Descriptive characteristics of the sample.

\begin{tabular}{|c|c|c|}
\hline & Males [N = B6] & Females $[N=197]$ \\
\hline Age [years] & $15.4 \pm 1.3$ & $15.6 \pm 1.4$ \\
\hline Height ICMV": & $1745 \pm 76$ & $165.4 \pm 5.9$ \\
\hline Weight 0 og/p." & $1063 \pm 16.1$ & $93.1 \pm 14.7$ \\
\hline BMv $0 \operatorname{sog}^{2} \mathrm{~m}^{2} \mathrm{~s}^{2}$ & $35.5 \pm 4.0$ & $33.9 \pm 4.4$ \\
\hline Tanner (pubertall Stage"w* & $45 \pm 0.5$ & $4.8 \pm 0.4$ \\
\hline TV riewing /hoursidgyl & $23 \pm 1.7$ & $2 A+2.1$ \\
\hline Compiser time |hoursiday) & $25 \pm 1.9$ & $22 \pm 20$ \\
\hline Video games (hours/daypos & $15 \pm 1.9$ & $0.2 \pm 06$ \\
\hline Screen time thourvidayp:" & $6.7 \pm 2.9$ & $52 \pm 2.8$ \\
\hline Intake in Detary Fat (An) & $34,4 \pm 6.4$ & $340 \pm 56$ \\
\hline Tocal calork hake (kcals)" & $2322 \pm 601$ & $2086=590$ \\
\hline Phyvical activiny score & $3.5 \pm 1.7$ & $3.4 \pm 1.7$ \\
\hline PA interaity-light ral & $\cos 6$ & 043 \\
\hline PA Intersity-moderate 190 & 29.1 & 33.2 \\
\hline$P A$ intensity vigorous & 23 & 2.5 \\
\hline Systolic BP $|\mathrm{mm} \mathrm{Hg}|^{* 2 .}$ & $1190 \pm 109$ & $111.3 \pm 9.1$ \\
\hline Disstolik UP (mm Hgl & $75.6 \pm 6.8$ & $744 \pm 73$ \\
\hline Thiglycerides immolu|" & $1.4 \pm 0.6$ & $12 \pm 0.6$ \\
\hline HDLC ImmelL/2: & $10 \pm 0.2$ & $1.1 \pm 0.3$ \\
\hline Lote immalu & $2.6 \pm 0.7$ & $26 \pm 0.7$ \\
\hline Toeal cholesterol Immollu & $42 \pm 0.8$ & $43 \pm 0.8$ \\
\hline Cholesterol/HDLC Ratiote. & $4.4 \pm 12$ & $3.9 \pm 1.0$ \\
\hline
\end{tabular}

Data are prewented as meins \pm standiud deviations except foe PA imensify which are in percentages: $\mathrm{PA}=$ Phrysical activity score of 1 = less than 5 minutes $2=5$ to 15 minuses, $3=15$ to 30 minuses, $4=30$ to 45 minutes, $5=45$ to 60 mimses, and 6 -Greater than 60 mixutes. Sex dfferences dotermined bry independent t-tests for continacus dets and dij-square for categorical data $\beta<05$

$\alpha \beta=05$

$x<01$,

doț 10.1371/joumal pone.0026643.105

or lipid measures, $\mathrm{PA}$ intensity was insersely correlated with systolic BP $(r=-0.12, p<0.05)$ and total cholesterol/HDL-C ratio $(r=-0.11, p<0.05)$. There were no significant associarions between total caloric intake (keals) or percent fat intake and $\mathrm{BP}$ and lipids, as shown in Tabke 2. Similarly, there was no significan relationship between percent protein or carbohydrate intake and BP and lipids (data not sbown), BMI correlated with BP and lipids more strongly than any oher of the covariates.

Table 3 shows the independent associations between the types of sedentary behaviour and intensity of PA and $\mathrm{BP}$ and lipids after adjustment for potential confounders. The adjusted $\mathrm{ex} \times$ sedentary behaviour interaction was nos significant foe any of the bealth outcomes. Similarly, the $\mathrm{sex} \times \mathrm{PA}$ duration and $\mathrm{xx} \times \mathrm{PA}$ intensity interactions were nox significant idata not shown'. However, after adjusting for age, sex, parental education, BMII, sexual maturity, total caloric intake, peroent of energy intake derived from dietary fat, duration of PA. and intensity of PA, the positive associations between video gaming and systolic BP (adjusted $x=0.13, \beta=1.1$, $\mathrm{p}<0.05$ ) and total cholesterol/ $\mathrm{HDL}$ ratio (arjusted $\mathrm{r}=0.12$, $\beta=0.10, p<0.05\rangle$ remained significant. The relationship between video gaming and trighycerides reached borderline significance (adjusted $r=0.11, \beta=0.04, p=07$, No oxher types of sedentary behaviour, including total screen time, were associated with BP or lipid measures after adjusting for conariates. After adjustment, PA 
Table 2. Correlations between Various Sedentary Behaviours, Physical Activity and Blood Pressure and Lipids.

\begin{tabular}{|c|c|c|c|c|c|c|c|c|c|c|c|c|c|c|}
\hline & TV & $C T$ & vo & ST & BMI & Age & $\operatorname{sex}$ & PE & ME & TS & Wafat & Cal & PA-1 & PA-D \\
\hline 5ystofic BP & 0.06 & $-a c \theta$ & 0.2004 & 0.07 & $0.25 *$ & $0.15 *$ & $-0.33^{*}$ & 0.06 & 0.01 & 0.01 & a.10 & a.10 & 0.12 & 0.03 \\
\hline Ciastolic BP & $a c 0$ & -0.04 & ans & $-0,02$ & $0.25 * *$ & Q.15** & -0.00 & 0.05 & $-\infty .01$ & a.on & 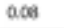 & 0.06 & -0.02 & 0.01 \\
\hline Total Cholestend & 0.03 & $-\infty, 1$ & 0.00 & 0.01 & -0.05 & 0.07 & 0.04 & -0.04 & 0.09 & 0.01 & 0.03 & 0,0 & -0.05 & $-0,04$ \\
\hline HDLC & -0.03 & 0.06 & $-0.19=4$ & -005 & $-0.5 \%$ & $0.14 *$ & $0.27 * *$ & 004 & $a 08$ & $0.19 * *$ & -0.04 & $-\infty 08$ & 0.10 & 0.04 \\
\hline Triglycerides & $a c e$ & $-\infty .00$ & 0.10 & 0.03 & $-a .23=$ & $0.10^{\circ}$ & $-0.12^{*}$ & -0.04 & -0.01 & $-a .12^{*}$ & 0.07 & 0.04 & -0.03 & -0.05 \\
\hline tac & 000 & $-\infty .01$ & 0.03 & 0.02 & 0.06 & acos & $-\infty, 01$ & -0.07 & -0.01 & $-0.12^{*}$ & 0.03 & 002 & -0.09 & $-0,9$ \\
\hline Total CholHOL-C & 0.04 & -0.04 & $0.19{ }^{\circ}$ & 0.08 & $0.25 *$ & -0.07 & $-0.23^{*}$ & -0.09 & -0.01 & $0.15 *$ & 0.06 & 0.07 & $-0.11^{*}$ & 0.09 \\
\hline
\end{tabular}

TV-television viewing in hours/dry, CT - Necreational compuder time in hours/day, VG = video games in hours/day, ST = screen time in hours/day, Sex $0=$ male,

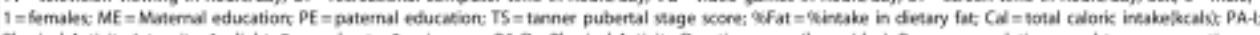

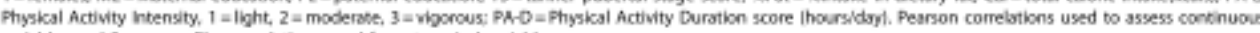
variables and Spearman Rho coendations used for concyorical variables. "p<.05

doi:10.1371/journalpone.0005643.1002

intensity was inversely associated with systolic BP (adjusted $r=-0.13, \quad \beta=-2.3, \quad p<0.05$ ), L.DL $-C$ (adjusted $r=-0.12$, $\beta=-0.15, p<0.05$, and total Cholesterol/HDL ratio [adjusted $r=-0.14, \beta=-0.28, p<0.05\}$ and positively associated with HDL $-\mathrm{C}$ (adjusted $r=0.11, \beta=0.05, p<0.05$ ). After adjustment, PA duration was only inversely associated with total cholesterol/ HDL $-\mathrm{C}$ ratio (adjusted $r=-0.11, \beta=-0,08, \mathrm{p}<0.05$ ).

\section{Discussion}

This sudy examined the independent association between duration of screen time and time spent in types of sedentary screen behaviours with IPP and lipids in overweight and ohese adolescents. Interestingly, total screen time was not associated with BP or lipids before or after adjusting for confounders. However, to our knowledge, we are the first to showe that time spent playing video games was the only type of sedentary behaviour associated with increased BP and lipids before and after adjusting for multiple confounders in a large sample of overweight and obese adolescents. Although males spent signifieantly more time playing video games than females, the sex $\times$ video game interaction was not significant, suggesting that the relationship between video games and BP and lipid outcomes did not differ by sex.

Only a few studies have examined the relationship berween types of screen time behaviours and chronic disease rivk factors in youth. In a large, nationally reperesentative sample of over 2500 children and adolescents from the 2003/4 and 2005/6 National Health and Nutrition Examination Survey (NHANES), Carson and Jansen [24] found that time spent watching TV was predictive of a higher score of an ageregated or clustered measure of cardio-metabolic risk, but recreational compoter time was not. Similarly, Martinez-Gomez et al. [25] found that TV viewing but not computer time was associated with increased BP in pres pubertal chiklren. Hossever, neither study assessed time spent playing seated video games, so it is uncertain how this form of seckentary behaviour would have predicted chronic discase risk compared to other sereen behaviours. In contrast to these findings, neither TV viewing nor recreational computer time was aseciated with $\mathrm{BP}$ or lipids in the current study, whether considering unadjusted or adjusted relationships. These discrepant findings are somewhat surprising since many sudies bave documented

Table 3. Independent Associations of Various Sedentary Behaviours, Physical Activity Intensity and Blood Pressure and Lipids.

\begin{tabular}{|c|c|c|c|c|c|}
\hline & TV & Cт & vo & ST & PA- Intensity \\
\hline Systelic BP & $0.48(-0.72$ to 1.7$\}$ & $-0.77(-1.860 .28)$ & $1.1 \mid 0.11$ to $\left.2.2\right|^{*}$ & $0.34 \mid-0.36$ to 1.00 & $-2.3(-0.3 \text { to }-4.3)^{\circ}$ \\
\hline Cisstolic BP & $-0.09(-0.21$ to 0.03$)$ & $-0.021-0.12$ to 0.09 & $-0.01(-0.12$ to 0.09$)$ & $-0.04\{-0.12$ to 0.031 & $-0.58[-2.1000 .90]$ \\
\hline Total Cholesterol & $-0.01(-0.11$ to 0.09$)$ & $-0.071-0.02$ to 0.16 & $0.03(-0.06000 .17)$ & $0.001-0.02$ to 0.10 & $-0.121-0.23$ to 0.05$)$ \\
\hline HDLC & $0.00\{-0.03$ to 0.03$\}$ & $0.01(-0.02$ to 0.04$)$ & $-0.02(-0.04$ to 0.01$)$ & $0.001-0.02$ to 0.024 & $0.050 .00001 .0 \mathrm{~F}$ \\
\hline Trighyerides & $0.05\{-0.03$ to 0.13$\}$ & $-0.04 \mid-0.11$ to 0.03$\}$ & $0.04(-0.03$ to 0.11$]$ & $0.021-0.03$ to 0.07 & $-0.05[-0.19$ to 0.065 \\
\hline 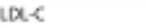 & $0.03(-0.12$ to 0.051 & $0.07(-0.01$ to 0.15$)$ & $0.02(-0.05$ to 0.101 & $0.031-0.02$ to 0.08 & -0.15 I-0.29 to $-\left.0.01\right|^{*}$ \\
\hline Total Cholhol-C & $-0.03(-0.16$ to 0.11$\}$ & $0.05(-0.06$ to 0.17$)$ & 0.100 .00 to $0.211^{\circ}$ & $0.07 \mid-0.01$ to 0.14$\}$ & -0.28 1-0.50 to $-0.061^{*}$ \\
\hline
\end{tabular}

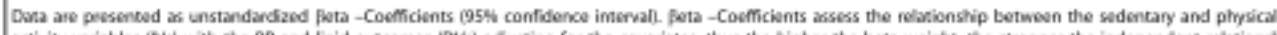
activity variables IVW with the BP and lipid ouscames IDWI acjusting for the covaristes thus the higher the beta weight, the stronger the independert relationship between the IVs and DVs, TV = tekevision viewing in hours/day, CT = recosesional computer time in hours/dy: VG = video gameplasing in hours/day; $S T=$ screen time in

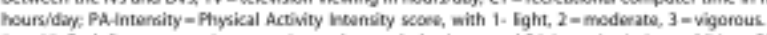

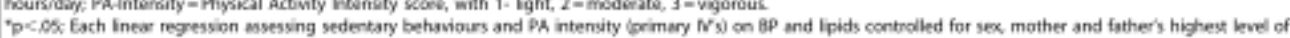

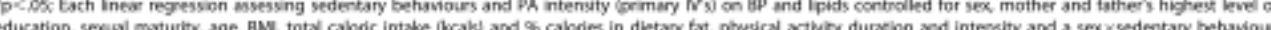

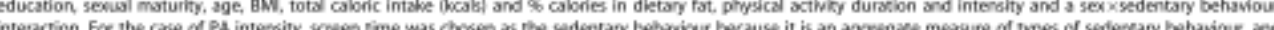

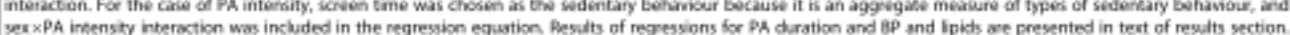
dol: $10.1371 / 90$ ind

Q. PLOS ONE I wwwplosone.org 
independent relationships berween TV viewing and böochemical markers of metabolic and CVD risk in children and adolescents [12-14,24], though not all have found these relationships [15]. The discrepant findings are unlikely to be due to differences in absolute time spent in either type of sedentary behaviour or variation since the mean values for time spent in TV and seated video gaming in the current study was similar, as was the variability. However, our sample was comprised of onerueight and obese adolescents rather than a nationally representative sample [24], so it is possible these differences in sample characteristics coukl explain, in part, the discrepant findings:

Given the nowelty of the findings, it is difficult to speculate what mechanisms may link video game use to high $\mathrm{BP}$ and more atherse lipid profiles however, a few possible explanations are offered. Similar to TV viewing, observational studies showed an association between video game playing and obesity in youth [19-

21]. Well-oontrolled crosover laboratory research indicates that vidoo game playing was associated with an increase in spontaneous food intake of energy dense snack foods compared to resting conditions $[22,23]$ which may have an adverse impact on obesity, BP and lipid profiles. Even though various dietary measures (percent of calories from fat and total caloric intake) were adjusted for in this sudy, residual confounding may have still been presents although unlikely given we found no association between food intake and the BP and lipid measures. In addition, laboratory studies have shown that seated video game playing acutcly causes increased heart rate, elevated systolic and diastolix BP, increased sympatbetic tone and mental workload compared to rest [22], perhaps due to the excitement, stress and concentration required for effective gaming. Given that these associations between seated video gaming and $\mathrm{BP}$ occurred in laboratory settings, it is possible that these effexts become exacerbated and more chronic when video games are played frequently oner time as observed in our study. It is also possible that the self-reporting of gaming was more accurate than other screen behaviours since gaming may be played in discrete bouts that are more distinct and memorable. whereas it may be more difficult to accurately quantify time spent watching TV or computer use because they may be more susceptible to periodic interruptions.

Similar to previous studies $[15,16,24,29]$. PA intensity in this study, primarily that performed at moderate-to-vigorous-intensity, was associated with lower BP and more favourable lipid profile before and after adjustment for confounders, including sedentary behaviour. However, duration of PA, defined as time spent in PA per day, was not associated with BP or lipids before adjustment, and was only associated with total cholesterol/HDL-C ratio after adjustment, indicating that $\mathrm{PA}$ intensity rather than duration of PA may be more dosely related to a lower BP and more favourable lipid profile in this popenlation. In addition, these findings highlight the notion that sedentary behaviour and PA are distinct constructs that may have different mechanisms in how they relate to healih outcomes [30].

\section{Limitations and Strengths}

This study has several strengths and limitations. We utilized a sample of onerweight and obese adokscents volunterring for exercise intervention, thus it is uncertain whether our findings can be generalized to overweight and obese adolescents in the community. In addition, time spent in sereen time behaviours and PA duration and intensity were measured by self-report which may introduce inaccuracies and hias in youth, whereby PA is generally over reported and sedentary behaviour is under reported [31], and it is possible that objective measures of these behaviours may have resulted in a different pattern of results. Anocher
Fimitation is the crose-sectional design, which limits the ability to make causal inferences ahout the relationships ohserved. Alko, makes spent more time video gaming than females, but maks only comprised about $30 \%$ of the sample, thus it is possible that sex differences between seated video games and $\mathrm{BP}$ and lipid profiles may have been detected if the sample was more balanced on sex, thus future rescarch is needed to test the veracity of this hypothesis. Regarding the imbalance in sex in the current study, it is poosible that males peroeive obesity to be a problem warranting intervention only at greater degrees of obesity, perhaps explaining. in part, that maks tended to have more adverse BP and lipoid profiles than females.

Strength of this study include an asessment of the three primary forms of sedentary screen time bebaviours (seated video games, TV and recreational computer time), whereas previous studies in the pediatric population examining the relationship between types of sedentary behaviour and risk factors of chronis disease only included TV and computer time $[17,24,25]$. Our shudy highlights the importance of measuring seated video gaming, which large sunves show has mass apoeal to teenagers [18], esperially males, and our dita also reflect the popularity of vides games gives time spent in gaming was comparable to TV viewing, Our study is also the first to examine the associations between sedentary screen time behaviours and BP and lipid profiles in an overweight and obese adolescent sample, who is at increased rish of CVD and premature mortality in adulthood compared to their normal weight peers $[8,9]$. Additionally, the present study included the broadest spexarum of BP and lipid measures as a proxy for assessing CVD risk factors compared to other studies [12-16] Assessing the full spectrum of $\mathrm{BP}$ and lipids is important because these CVD risk factors in adoleseence track into adolthood [7], and brant dixase and stroke are still leacling causes of morbifity and premature mortality [32]. Lastly, the current study included a comprehensive set of covariates that statistically controlled for several confounding variables, strengthening the internal validity of the findings.

\section{Conclusions}

To the best of our knowledge, the present study is the firs to demonstrate that seated video gaming is associated with increased $\mathrm{BP}$ and lipoids in a sample of oveneight and obese adolescents. after controlling for adiposicy, caloric intake, dietary fat intake, PA duration and intensity and several otber important confounders. Our results prowide further support for the public health guidelines recommending that children and youth limit their sedentary behaviour, especially screen time behaviours [33,34]. Our findings provide support for reducing time spent playing seated vides games as a possible means to promote health and prevent CVD in this high risk group of onerweight and obese adolescents. Future research is needed to first replicate these novel findings and subecquently aim to elucilate the mechanisms linking seated video gaming and elevated $\mathrm{BP}$ and lipoids in a high risk sample of overweight and obese youth.

\section{Acknowledgments}

The authar would like to thank all the sulyjects wbo participated in the tubly and all the rexarch saf utu asisicd with data collection and implementation of the protecol.

\section{Author Contributions}

Conceived and designed the experiments GSG GPK SH JM DP RG TJS MST RJS. Performed the experiments PP ASA. Analyand the data: GSG. Wrote the paper: GSG GPK SH PP ASA TJS MET JM DP RG RJS. 


\section{References}

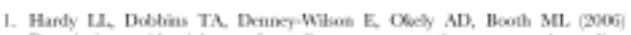
Descripche epdetemiodogy of small screes recreation antong Australian

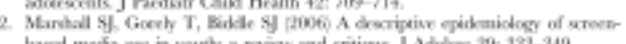

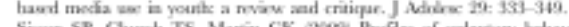

3. Simun SR, Church TS, Marin CK (2009) Prufiles of sndertary behasice in

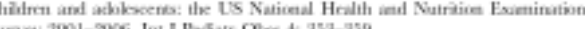

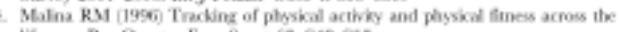
IGspas. Res Qaaner Exer 5pon 67: S45-50?

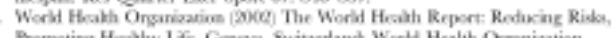

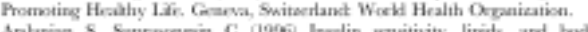

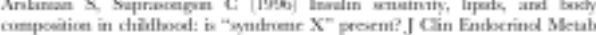
31: $1009-1062$.

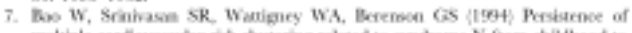

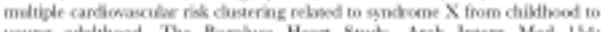

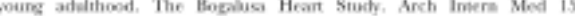
1342-1845.

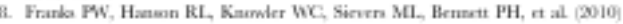

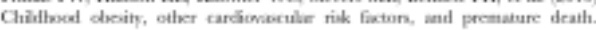

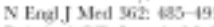

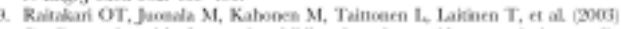

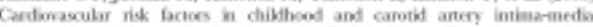

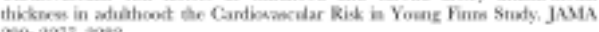
$200 \mathrm{t} 2273-2239$

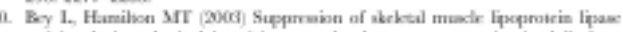

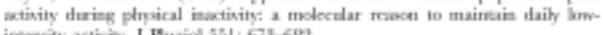
incensty stim . F.

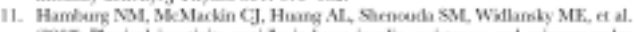
3007) Firsical mactivity repidy induces houln reskiance and microvascular

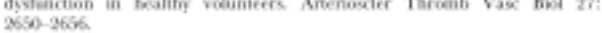

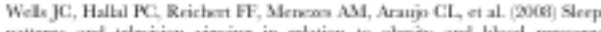

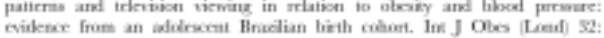
10+2-1019.

13. Weng ND, Hei TK, Qquendah N, Daviden DM, Rusin St, et al. (1992)

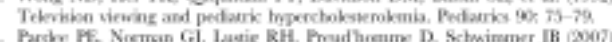

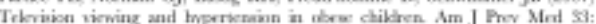
439-44.

15. Bbehund U, Brage S, Friberg K, Harro M, Androum SA, a al. (2005) TV

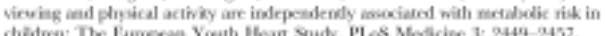

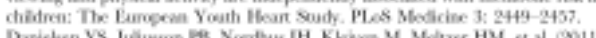

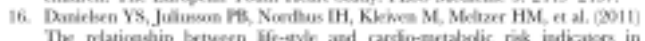

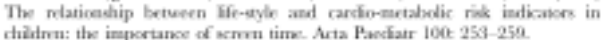

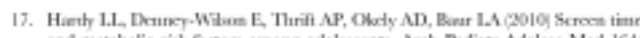

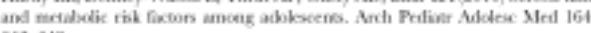

8. Jrakin H (2009) Art from the tightal agr. Techndugical Rer 102 117-120.

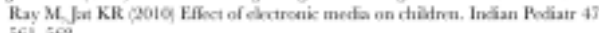

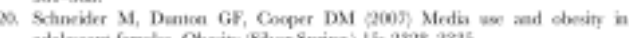

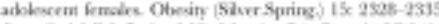

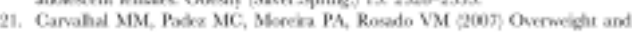

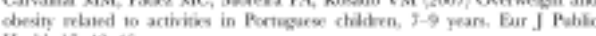

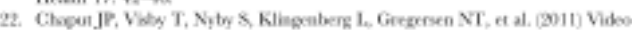

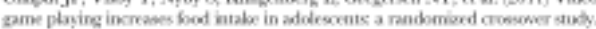
zame plaging increases bod inta
An J Cla Nutr $10: 1196-201$

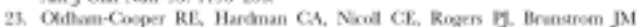

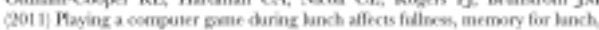

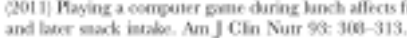

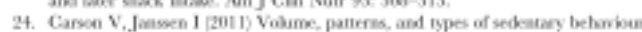

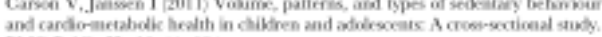
BMC: Putilic Heath 11-274.

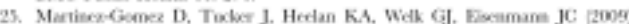

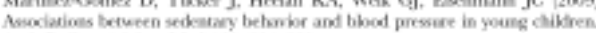
Ach Pedar Mblose Med 16: $724-790$.

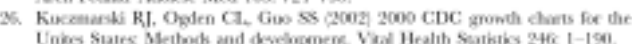

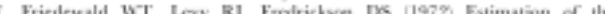

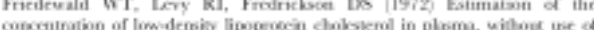

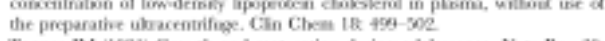

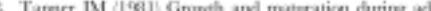
43-5.

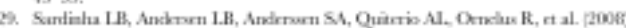

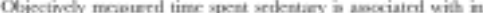

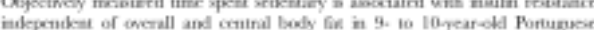
diaken. Disters Care 51 : 569-575.

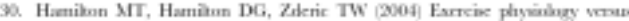

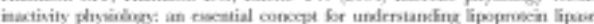
regulation. Ever Spers Sci Rev 32. 161-166

31. Adamo KB, Prince SA, Trims AC, Cumber-Gurter S, Tremblhy MS $(2000)$ A

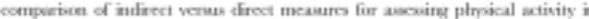

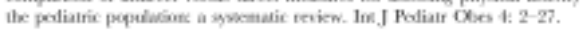

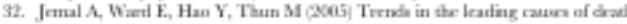
in the Unitrd Stairs, 1970-2002. Jisin 294: 1250-1259.

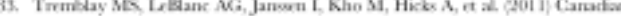

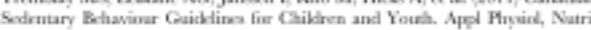
Mrtalued 36 $39-64$

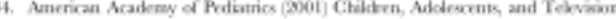
Pociatr 107x: 423-428. 


\section{The association between accelerometer-measured patterns of sedentary time and health risk in children and youth: results from the Canadian Health Measures Survey}

Rachel C Colley ${ }^{12}$, Didier Garriguet', Ian Janssen ${ }^{3,4}$, Suzy L Wong', Travis J Saunders ${ }^{2}$, Valerie Carson ${ }^{3}$ and Mark S Tremblay ${ }^{2}$

Abstract
Background: Self-reported screen time is associated with elevated health risk in children and youth; however, research examining the relationship between accelerometer-measured sedentary time and health risk has reported mixed findings. The purpose of this study was to examine the association between accelerometer-measured patterns of sedentary time and health risk in children and youth.

Methods: The results are based on 1,608 children and youth aged 6 to 19 years from the Canadian Health Measures Survey (2007-2009). Sedentary time was measured using the Actical accelerometer. Breaks in sedentany time and prolonged bouts of sedentary time lasting 20 to 120 minutes were derived for all days, weekend days and during the after-school period (li.e, after 3 pm on weekdays). Regression analyses were used to examine the association between patterns of sedentary time and body mass index (BMI), waist circumference, blood pressure and non-HDL cholesterol.

Results: Boys accumulated more sedentary time on weekdays after $3 \mathrm{pm}$ and had a higher number of breaks in sedentary time compared to girls. Overweight/cbese boys (aged $6-19$ years) accumulated more sedentary time after 3 pm on weekdays (282 v5. 259 min, $p<.05$ ) and as prolonged bouts lasting at least 80 minutes (171 v5. 133 min, $\mathrm{p}<.05$ ) compared to boys who were neither overweight nor obese. Prolonged bouts of sedentary time lasting at least 80 minutes accumulated after $3 \mathrm{pm}$ on weekdays were positively associated with BMI and waist circumference in boys aged 11-14 years ( $\mathrm{p}<006$ ). Each additional 60 min of sedentary time after 3 pm on weekdays was associated with a $1.4 \mathrm{kgm}^{-2}$ higher BMI and a $3.4 \mathrm{~cm}$ higher waist circumference in 11-14 year old boys. No sedentary pattern variables differed between girls who were not overweight or obese and those who were overweightobese and none of the sedentary pattern variables were associated with any health markers in girls.

Conclusions: The findings confirm results of other studies that reported accelerometer-measured sedentary time was not associated with health risk in children and youth. Even when the pattern and timing of sedentary time was examined relative to health markers, few associations emerged and were limited to boys aged 11-14 years.

Keywords: Behaviour, Breaks, Bouts, Physical activity, Pediatric

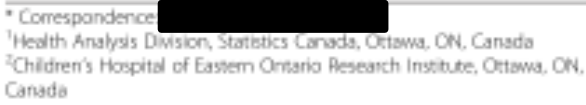




\section{Background}

The association between sedentary behaviour and health risk in children appears to be influenced by how the sedentary behaviour is measured, defined and categorized. Several studies have reported significant associations between self-reported screen time and increased risk of obesity and cardio-metabolic disease risk in children [1-4]. However, screen time provides a limited perspective on total sedentary time because it is only a sub-component of a behaviour that is defined as encompassing "any waking behaviour characterized by an energy expenditure $s 1.5$ METs while in a sitting or reclining position" [5]. Further, self-reported data relating to lifestyle habits may be limited by bias and recall difficulties $[6,7]$.

Accelerometers are now commonly used to objectively measure total sedentary time, and have the capacity to also derive the pattern and timing in which it is accumulated. In contrast to self-report, associations between accelerometer-measured sedentary time and health risk in children have been mixed with some studies reporting significant associations [8-10] and others not [1,11-16]. Studies that collected both questionnaire and accelerometer data on sedentary behaviour/time found an association between self- or parent-reported screen time and health risk but no association between accelerometermeasured sedentary time and health risk $[1,14,15]$. The only studies reporting significant associations between accelerometer-measured sedentary time and health risk did not adjust for moderate-to-vigorous physical activity (MVPA) [8-10] or reported that the significant associations existed in unadjusted models but were attenuated when MVPA was controlled for [13,16]. Some engagement in sedentary behaviour is inevitable in the day (e.g., eating, relaxing, homework, school etc.); however, it is presently unknown how much sedentary time is too much. Inevitably, there is variation between people in the length of time they engage in sedentary behaviour bouts (i.e, prolonged periods of sedentary time) and how often these bouts are interrupted by activity. Research in adults suggests that the pattern of accumulation of sedentary time is important to consider in relation to health risk.

The inconsistent findings between accelerometermeasured sedentary time and health risk among children and youth have led to the examination of more sophisticated sedentary time variables. For example, it has been proposed that the pattern in which sedentary time is accumulated may provide insight beyond what has been observed to-date using the total volume of sedentary time [17]. Others have attempted this approach in adults and children; however, the sedentary pattern variables have been limited to breaks or interruptions in sedentary time [17] and engagement in prolonged bouts of sedentary time lasting up to 30 minutes [1]. Further, studies examining how the pattern of sedentary time relates to health risk in children have not considered the importance of periods of discretionary free time separate from the whole day in children [18-20]. The present study sought to build upon this work by extending the length of the prolonged bouts up to 2 hours and by examining these variables during periods when children and youth typically have free time. In other words, this study sought to identify novel sedentary pattern variables that were more representative of how children and youth typically engage in sedentary behaviour.

The purpose of this study was to examine the association between accelerometer-measured patterns of sedentary time and health risk in children and youth. Specifically, this study examines whether breaks in sedentary time and sedentary time accumulated as prolonged bouts during periods of discretionary free time in children (i.e., after-school and weekends) have stronger associations with health risk in children when compared to average daily sedentary time across the week. We hypothesize that sedentary time accumulated during periods of discretionary free time will better discriminate between children engaging in healthy and unhealthy levels of sedentary behaviour when compared to simply examining overall sedentary time.

\section{Methods \\ Data source}

The Canadian Health Measures Survey (CHMS) collected data from a nationally representative sample of the population aged 6 to 79 years living in private households at the time of the survey. Data were collected at 15 sites across Canada from March 2007 through February 2009. Ethics approval was obtained from Health Canada's Research Ethics Board [21]. For children aged 6-13 years, written informed consent was obtained from a parent or legal guardian, in addition to written informed assent from the child; youth aged $\geq 14$ years provided independent consent. Of the households selected, $69.6 \%$ agreed to participate. Of that group, $88.5 \%$ of the selected 6-19 year olds completed a questionnaire and $86.9 \%$ of this group participated in the mobile examination centre component. Of the children and youth who agreed to wear the accelerometer and returned the device, $87.4 \%$ had at least one valid day of data, and $76.3 \%$ had at least four valid days. These multiple stages of response can be multiplied together $(69.6 \% \times 88.5 \% \times$ $86.9 \% \times 76.39 \%$ ) to provide an overall response rate of $40.8 \%$. Adjustments were made at each stage to manage any potential non-response bias. The data were then weighted to be representative of the Canadian population. More extensive details of the CHMS [22] and direct measurement of physical activity in the CHMS [23,24] are available elsewhere. 
Study procedures

Upon completion of the mobile examination centre visit. ambulatory respondents were asked to wear an Actical accelerometer (Phillips - Respironics, Oregon, USA) over their right hip on an elasticized belt during their waking hours for seven consecutive days, except when the device could get wet. The Actical measures and records time-stamped acceleration in all directions, providing an index of physical activity intensity. The Actical has been validated to measure physical activity in children [25] and cut-points for sedentary intensity have been proposed for children [26]. The accelerometers were initialized to collect data in 60 -sec epochs.

\section{Accelerometer data reduction}

Participants aged 6 to 19 years with four or more valid days [24], one of which was a weekend day, were included in this analysis (Table 1). A valid day was defined as having 10 or more hours of wear time [24]. Wear time was determined by subtracting non-wear time from 24 hours. Non-wear time was defined as at least 60 consecutive minutes of zero counts, with allowance for two minutes of counts between zero and 100 [24]. For each minute, the level of movement intensity was based on cut-points corresponding to intensity level: sedentary $=<$ 100 counts per minute (cpm) [26]; MVPA = $\geq 1,500 \mathrm{cpm}$ [25]. Minutes of MVPA and sedentary time were summed for each day for each participant.

\section{Sedentary time variables}

Sedentary time was calculated for all days, weekdays and weekend days. The total number of breaks in sedentary time was summed for each valid day and then averaged across the week, weekdays and weekend days. A break was considered as an interruption in sedentary time (lasting a minimum of one minute) in which there was a transition in accelerometer count from $<100 \mathrm{cpm}$ to $z$ $100 \mathrm{cpm}$.

To be defined as a prolonged sedentary bout, there had to be $280 \%$ of minutes below the $100 \mathrm{cpm}$ cut-point (eg, 16 out of 20 minutes or 32 out of 40 minutes) [1] The bout stopped when $\angle 80 \%$ was below the $100 \mathrm{cpm}$ cut-point or when there were 23 consecutive minutes $2100 \mathrm{cpm}$ or any observations $21500 \mathrm{cpm}$ (cut-point for moderate intensity). Sedentary bouts lasting at least 20 , $40,60,80,100,120$ minutes were derived using this approach. Multiple lengths of sedentary bouts were derived to reflect a range of different sedentary behaviours such as watching a television show ( 30 minutes), watching a movie (1.5-2 hours), or playing video games (anywhere between 20 minutes and 2 hours). The choice of $800 \%$ as the criteria for sedentary minutes within a bout was purposeful to mimic real-world situations where largely sedentary pursuits (eg, watching TV, doing homework) are often occasionally interrupted with light activity (e.g., to go to washroom, answer the phone, get a snack etc.).

\section{Body mass index and waist circumference}

Height was measured to the nearest $0.1 \mathrm{~cm}$ using a ProScale M150 digital stadiometer (Accurate Technology Inc., Fletcher, USA) and weight was measured to the nearest $0.1 \mathrm{~kg}$ with a Mettler Toledo VLC with Panther Plus terminal scale (Mettler Toledo Canada, Mississauga, Canada). BMI was calculated as weight ( $\mathrm{kg}$ ) divided by height squared $\left(\mathrm{m}^{2}\right)$. Children were categorized as not overweight/obese (which includes underweight and healthy weight) or overweight/obese according to ageand sex-specific cut-points [27]. Waist circumference was measured with a stretch-resistant anthropometric tape at the end of a normal expiration to the nearest $0.1 \mathrm{~cm}$ at the mid-point between the last rib and the top of the iliac crest [28].

\section{Blood pressure}

Systolic and diastolic blood pressure were measured with the BpTRU ${ }^{\mathrm{Tu}}$ BP-300 device (BpTRU Medical Devices Ltd., Coquitlam, British Columbia); an automated and validated $[29,30]$ electronic monitor that uses an upper arm cuff. Six measurements were taken at 1-min intervals with the last 5 measurements used to calculate

Table 1 Descriptive characteristics of the sample (mean \pm standard deviation)

\begin{tabular}{|c|c|c|c|c|c|c|}
\hline & \multicolumn{3}{|c|}{ Boys } & \multicolumn{3}{|c|}{ Girls } \\
\hline & 6 to 10 years & 11 to 14 years & 15 to 19 years & 6 to 10 years & 11 to 14 years & 15 to 19 years \\
\hline Total sample \{n\} & 369 & 256 & 184 & 390 & 248 & 211 \\
\hline Age (vears) & $8.2 \pm 1.4$ & $12.5 \pm 1.0$ & $17.0 \pm 15$ & $8.1 \pm 1.3$ & $123 \pm 1.1$ & $169 \pm 0.1$ \\
\hline Height (cm) & $133.9 \pm 10.4$ & $1589 \pm 11.1$ & $175.6 \pm 7.6$ & $131.6 \pm 10.3$ & $156.9 \pm 78$ & $1662 \pm 6.7$ \\
\hline Weight $0 \mathrm{kgl}$ & $32.5 \pm 9.4$ & $52.1 \pm 147$ & $72.4 \pm 18.1$ & $299 \pm 89$ & $506 \pm 11.6$ & $62.5 \pm 13.8$ \\
\hline EW $\left(\mathrm{kg} / \mathrm{m}^{2}\right)$ & $178 \pm 3.1$ & $20.3 \pm 3.9$ & $23.4 \pm 5.0$ & $17.0 \pm 3.1$ & $20.4 \pm 3.8$ & $226 \pm 44$ \\
\hline Waist circumference (cm) & $61.1 \pm 9.8$ & $706 \pm 10.9$ & $80.1 \pm 12.9$ & $57.9 \pm 8.5$ & $70.1 \pm 100$ & $75.4 \pm 109$ \\
\hline MUPA (average mind ${ }^{-1}$ ) & $69.4 \pm 29.1$ & $59.5 \pm 29.4$ & $53.1 \pm 25.9$ & $58.1 \pm 22.6$ & $472 \pm 246$ & $39.1 \pm 230$ \\
\hline
\end{tabular}

aw body mass index

MVPA moderate-to-vigonous phrysical activing. 
average blood pressure and heart rate [29]. The device automatically inflates and deflates the cuff and uses an oscillometric technique to calculate systolic and diastolic blood pressure.

\section{Non-HDL-Cholesterol}

Non-HDL-cholesterol was calculated by subtracting HDL cholesterol, measured using a non-HDL precipitation method on the Vitros 5,1FS (Ortho Clinical Diagnostics), from total cholesterol [31]. Non-HDL. cholesterol consists of very low density, low density, and intermediate density lipoprotein cholesterol and therefore reflects the cholesterol content of all apo B containing lipoproteins. Non$\mathrm{HDL}$ cholesterol was chosen as the lipid marker because it is an important indicator of cardiovascular and diabetes risk among children and adolescents and is not reliant upon a fasted blood sample [32]. Blood samples were taken by a certified phlebotomist and were analyzed at the Health Canada Laboratory (Bureau of Nutritional Sciences, Nutrition Research Division), Other blood markers are available in the CHMS; however, the fasting requirement for some of these measures resulted in a marked re. duction in the sample size when they are included. To ensure we had appropriate power for the primary purpose of this analysis, we included non-HDL-cholesterol as the sole blood marker.

\section{Statistical analysis}

Differences between sex, age groups and BMI status were assessed using t-tests. Statistical significance was set at a $p$ value of 0.05 . It is important to note that the values presented from this analysis in Figures 1 and 2 represent the mean across the week for sedentary time accumulated in prolonged bouts. In other words, there are zeros included in the averaging (because not all individuals had bouts of each length on all days) which in the case of 120 minutes bouts, brings the mean time accumulated below 120 minutes.

Associations between sedentary time variables and BMI and waist circumference were assessed using regression analyses. BMI and waist circumference vary by sex and change with normal growth and maturation [27,33]. Age and sex were both significantly correlated with average daily minutes of MVPA and age was significantly correlated with average daily minutes of sedentary time. Therefore, all regression analyses were completed separately by sex and the following age categories: 6-10, 11-14 and 15 to 19 years. The choice of age categories was based on the sampling design of the CHMS. Linear regression models were run separately for each sex and age grouping and were adjusted for age, average daily minutes of MVPA on valid days, and accelerometer wear time. The wear time adjustment was specific to the time period being examined. For example, the models for weekdays after $3 \mathrm{pm}$ where adjusted for wear time on weekdays after $3 \mathrm{pm}$. Eight separate regression models (sedentary time, breaks in sedentary time, prolonged bouts lasting at least $20,40,60,80,100,120$ minutes) were run for each time period: overall, weekdays after $3 \mathrm{pm}$ and weekends. The $p$-value to reach statistical significance in the linear regression analyses was adjusted for the number of models run. In other words, to reach statistical significance, the regression $p$ values had to be less than .006 (i.e., $0.05 / 8=0.006$ ).

All statistical analyses were performed using SAS v9.1 (SAS Institute, Cary, NC) and were based on weighted. data (to be representative of the Canadian population and to account for non-response bias) for respondents with at least four valid days. To account for survey design effects of the CHMS, standard errors, coefficients of

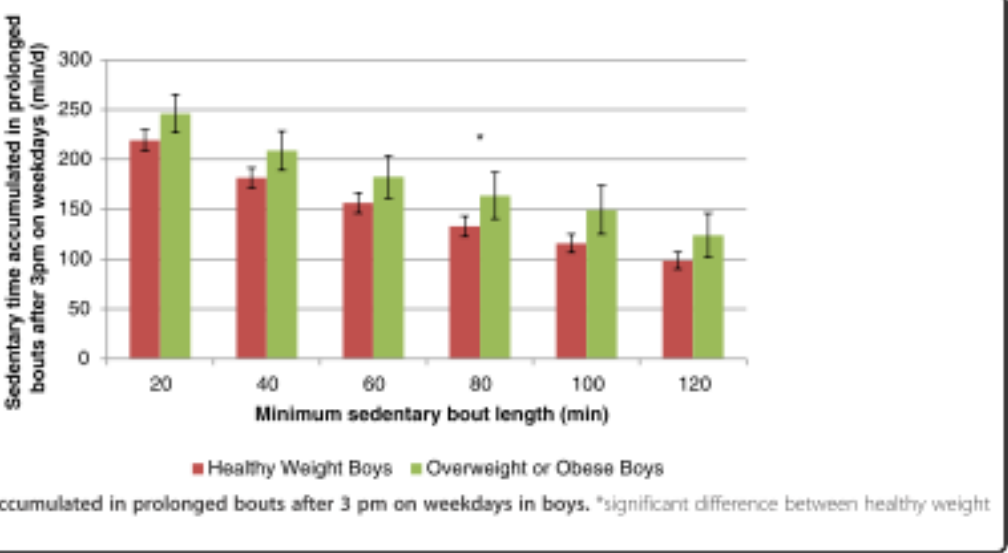




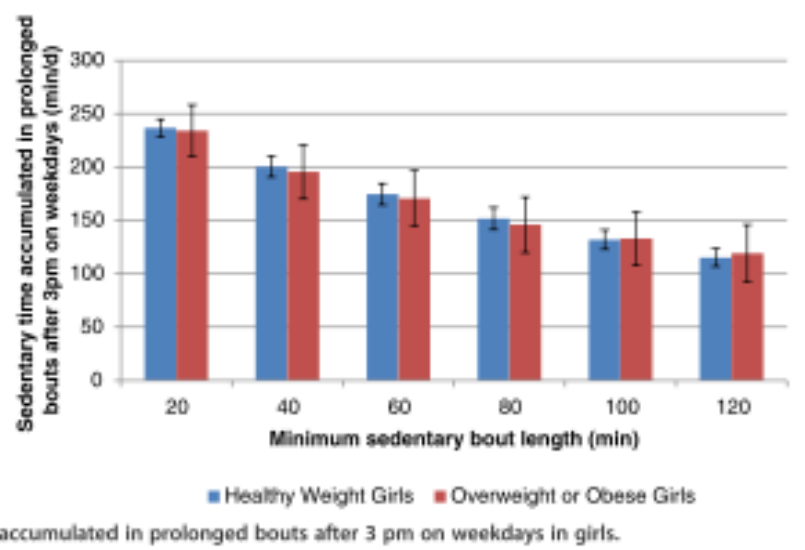

variation, and $95 \%$ confidence intervals were estimated using the bootstrap technique [34-36]. Overweight and obese were collapsed into one category because we lacked statistical power to compare not overweight; obese (includes healthy weight and underweight), overweight and obese as 3 separate categories.

\section{Results}

Descriptive characteristics of the sample are provided in Table 1. The analysis is based on 1,608 children and youth between the ages of 6 and 19 years. The sex split was even between boys $(\mathrm{n}=809,50.3 \%)$ and girls $(n=799)$.

\section{Sex and Age differences}

Sex and age differences are presented in Table 2. On average, boys accumulated 508 minutes per day of sedentary time while girls accumulated 524 minutes per day. Boys accumulated more sedentary time on weekdays after $3 \mathrm{pm}$ compared to girls, while boys had a lower number of breaks in sedentary time per day com. pared to girls. Sedentary time was higher in 11-14 year olds and 15-19 year olds compared to children aged 610 years. Girls aged $15-19$ years accumulated more sedentary time overall and after school compared to boys of the same age.

\section{Body mass index differences}

Differences by BMI status are represented graphically in Figure 1 for boys and in Figure 2 for girls. Overweight and obese boys accumulated more sedentary time after $3 \mathrm{pm}$ on weekdays when compared to boys who are not overweight/obese (Table 2). Overweight and obese boys accumulated more sedentary time after $3 \mathrm{pm}$ on weekdays as prolonged bouts lasting at least 80 minutes when compared to boys who are not overweight/obese (171 vs. 133 min. $^{-1}$ ) (Figure 1). No sedentary time variables differed between girls who are overweight/obese and those who are not overweight or obese (Table 2; Figure 2).

\section{Regression analysis results}

Prolonged bouts of sedentary time lasting at least 40 . minutes, after $3 \mathrm{pm}$ on weekdays, were positively associated with waist circumference $(\mathrm{B}=2.23, p<.006)$ while prolonged bouts of sedentary time lasting at least 80 minutes was positively associated with both BMI $(B=0.72$, $p<.006)$ and waist circumference ( $B=1.76, p<.006)$ in boys aged 11-14 years. Each additional 60 minutes of sedentary time accumulated during the after school period was associated with a $1.4 \mathrm{~kg} \mathrm{~m}^{-2}$ higher BMI and a $3.4 \mathrm{~cm}$ higher waist circumference in 11-14 year old boys. Number of breaks in sedentary time, after $3 \mathrm{pm}$ on weekdays, was negatively associated with waist circumference $(B=-$ $4.04, p<.006)$ in boys aged 11-14 years. No sedentary time variables were significantly associated with BMI or waist circumference in girls of any age or in boys aged 610 or 15-19 years. No sedentary time variables were associated with blood pressure or non-HDL cholesterol in boys or girls. The full results from the regression analyses are available as Additional file 1: Tables S1, Additional file 2: Tables S2, Additional file 3: Tables S3, Additional file 4: Tables S4, Additional file 5: Tables S5.

\section{Discussion}

The objective of this study was to examine the association between accelerometer-measured sedentary time and health risk in children. Our analysis supports previous studies that found few or no significant associations between accelerometer-measured sedentary time and health risk in children $[1,11-16]$. This study is novel because it 
Table 2 Descriptive sedentary time results (mean \pm standard deviation), by age, sex and obesity status

\begin{tabular}{|c|c|c|c|c|}
\hline & $\begin{array}{l}\text { Sedentary time } \\
{[\text { [min/d }]}\end{array}$ & $\begin{array}{l}\text { Sedentary time on weekdays after } \\
\qquad 3 \mathrm{pm}(\mathrm{min} / \mathrm{d})\end{array}$ & $\begin{array}{l}\text { Sedentary time on } \\
\text { weekends (min/d) }\end{array}$ & $\begin{array}{c}\text { Brealks in sedentary time per day } \\
\text { (number/d) }\end{array}$ \\
\hline Boys & $507.5 \pm 90.8$ & $265.5 \pm 65.8^{\prime}$ & $490.9 \pm 114.5$ & $81.2 \pm 11.6^{\circ}$ \\
\hline \multicolumn{5}{|l|}{ Age } \\
\hline 6 to 10 years & $465.5 \pm 79.5$ & $2128 \pm 520$ & $40.5 \pm 101.1$ & $84.4 \pm 10.3^{*}$ \\
\hline 11 to 14 years & $524.1 \pm 769^{*}$ & $2739 \pm 580^{\circ-}$ & $5032 \pm 1033^{-4}$ & $792 \pm 11.2^{* * *}$ \\
\hline 15 to 19 years & $5539 \pm 770^{\circ}$ & $3107 \pm 447^{10}$ & $5338 \pm 126.4$ & $80.1 \pm 13.6^{\prime \prime}$ \\
\hline \multicolumn{5}{|l|}{$8 / \pi$} \\
\hline $\begin{array}{l}\text { Not overweight or } \\
\text { obese }\end{array}$ & $499.9 \pm 89.4$ & $259.4 \pm 63.7$ & $483.7 \pm 1150$ & $812 \pm 11.3$ \\
\hline $\begin{array}{l}\text { Orenweight or } \\
\text { obese }\end{array}$ & $5278 \pm 946$ & $2817 \pm 69.2^{-*}$ & $509.7 \pm 22000$ & $81.1 \pm 12.6$ \\
\hline Girls & $523.8 \pm 91.6$ & $277.1 \pm 67.5$ & $493.6 \pm 106.7$ & $85.4 \pm 11.7$ \\
\hline \multicolumn{5}{|l|}{ Age } \\
\hline 6 to 10 years & $466.1 \pm 729$ & $215.4 \pm 521$ & $428.7 \pm 960$ & $896 \pm 10.4$ \\
\hline 11 to 14 years & $5268 \pm 63.5^{\prime \prime}$ & $275.0 \pm 49.3^{\circ}$ & $5032 \pm 875^{-}$ & $847 \pm 105^{-}$ \\
\hline 15 to 19 years & $582.1 \pm 81.4^{* *}$ & $3263 \pm 47.5^{\circ}$ & $538.4 \pm 106.1^{--}$ & $82.6 \pm 13.6^{-}$ \\
\hline \multicolumn{5}{|l|}{ BM } \\
\hline $\begin{array}{l}\text { Not ovenweight of } \\
\text { obese }\end{array}$ & $523.5 \pm 91.3$ & $277.1 \pm 68.3$ & $495.4 \pm 107.2$ & $852 \pm 11.5$ \\
\hline $\begin{array}{l}\text { Overweight or } \\
\text { obese }\end{array}$ & $5246 \pm 93.1$ & $2770 \pm 64.2$ & $487.1 \pm 1050$ & $862 \pm 12.5$ \\
\hline
\end{tabular}

sigrificantly d flerens to estimate for ginls $\{p<.05\}$.

significantly diflerem to entimate for $6-10$ year olds of same sex $|p<05|$ - significantly difleremt to estimate for not overweight or obese $|p<05|$. aM, body mans inden.

included a wider range of sedentary time variables than what has been previously considered that characterize the timing and patterning of how the sedentary time is accumulated. Further, the sedentary pattern variables were designed to be more reflective of real-world sedentary be haviour. For example, a limited number of short transitions into light activity were allowed to reflect real-life situations where individuals are sedentary for long periods but move around occasionally (i.e., to go to the washroom or answer the phone). Despite the inclusion of more comprehensive sedentary pattern variables, this study found few significant relationships with health risk and the associations observed were limited to boys aged 11-14 years.

In theory, excessive sedentary time is associated with negative health outcomes $[4,37]$ and self-reported screen time is associated with elevated health risk in children [1-3]; however, the way we currently measure sedentary time with accelerometers does not consistently support this link. To date, the research linking accelerometermeasured sedentary time with health risk among children and youth has been mixed. It is therefore unclear whether a relationship exists only in some populations or if differences in analytical approaches explain the inconsistencies observed. There appears to be more evidence supporting a lack of relationship between accelerometer-measured sedentary time and health risk in children and youth [1,11-16] than there is supporting a relationship [8-10]. Adjustment for MVPA appears to attenuate significant associations between accelerometer-measured sedentary time and health risk $[13,16]$, suggesting that MVPA is more powerful than total sedentary time at explaining the variance in health risk in children and youth. In our unadjusted regression models, sedentary time was associated with BMI and waist circumference in boys aged 6 to 14 and girls aged 6 to 10 years; however, after adjustment for MVPA, these associations remained significant only in 11-14 year old boys.

In 2008, Healy and colleagues published a paper that reported a significant association between number of daily breaks in accelerometer-measured sedentary time and lower metabolic risk in adults [17]. This work led researchers to question whether it is the pattern of how sedentary time is accumulated, rather than simply the total volume of sedentary time, which matters for health. Do frequent interruptions in sedentary time attenuate the health risk that sedentary time imposes? Does this relationship apply in both children and adults? Carson and Janssen found no significant associations between breaks in sedentary time or prolonged bouts of sedentary time lasting 30 minutes with cardio-metabolic risk 
factors in a large sample of American children [1]. Number of breaks in sedentary time was only associated with waist circumference in 11-14 year old boys in the present analysis. We included an additional layer of complexity by examining sedentary time, breaks and prolonged bouts of sedentary time during periods of discretionary free time: weekends and after school. We hypothesized that sedentary time accumulated during these periods would better discriminate between children engaging in healthy and unheslthy levels of sedentary behaviour when compared to simply examining overall sedentary time. We observed no significant associations between the patterns of sedentary time accumulated on weekends and health risk in children; however some relationships emerged when we examined sedentary time accumulated during the after school period. Interestingly, we only observed significant associations in boys aged 11 to 14 years of age when the regression models were adjusted for age, MVPA and accelerometer wear time-

It is difficult to speculate why we observed significant findings in boys and not girls. It is possible that more overweight and obese boys in this sample were engaging in prolonged bouts of sedentary time after school, a finding consistent with previous research that has found that boys spend considerably more time in specific sedentary behaviours such as video game playing [38-40]. Average daily sedentary time and weekend sedentary time did not differ between boys and girls while sedentary time accumulated after $3 \mathrm{pm}$ on weekdays was higher in boys compared to girls ( 277 vs. 266 minutes). Significant differences between boys who are not overweight/obese and overweight/obese boys were observed in the sedentary variables; however, no such differences were observed in girls. For example, there was virtually no difference in average daily sedentary time between girls who are not overweight/obese versus those who are ( 524 vs. 525 min. $d^{-1}$ ) while a more marked difference existed between boys who are not overweight/obese versus those who are ( 500 vs. $528 \mathrm{~min}^{-1} \mathrm{~d}^{-1}$. In Figure 1, a distinction between boys who are not overweight/obese and those who are can be observed across all bout lengths; however the difference is only statistically significant when the bout length is at least 80 minutes long. By comparison, no difference is noticeable by overweight/obesity status in girls and there is more crossover in the error bars in girls (Figure 2). Similarly, no significant associations emerged in girls in the regression analyses while in 11-14 year old boys, prolonged bouts of sedentary time lasting at least 80 minutes, accumulated during the after school period were associated with both BMI and waist circumference. Explaining why sig nificant associations were observed in 11-14 year old boys but not those who were $6-10$ or $15-19$ years is not easy. In a large sample of US children, Sisson and colleagues observed an increase in screen time with age from 2 to 15 years [41]. In the Health Behaviour and School Aged Children Survey, the Canadian data show that screen time increases from age 11 to 15 years [42] with the peak occurring in grade 9 (approximately 14 . years) [43]. These large data sets suggest that the 1114 year old age group may be an age range where screen time habits change significantly, thus increasing the amount of variation (and likelihood to find significant associations) in this variable.

The lack of evidence linking accelerometer-measured sedentary time with health risk in children is counterintuitive given the consistent observation that screen time, a key contributor to total sedentary time, is associated with health risk [1-4]. One of the fundamental differences between self-reported screen time and objectively measured sedentary time is that the former is capturing one specific activity while the latter is capturing screen time in addition to many other sedentary behaviours. Much of the time accumulated as "sedentary" represents normal aspects of day-to-day life therefore capturing every minute in a day that is sedentary, as accelerometers do, may dilute the associations between specific sedentary behaviours (e.g., watching television) and health risk. It is possible that some forms of sedentary behavior (e.g., screen time, long car or bus travel) are associated with negative health outcomes while other forms of sedentary behavior (e.g., eating, reading, resting. socializing etc.) are not. Similarly, data reduction procedures used in accelerometry analysis (e.g., 10 hour wear time criteria) were developed to accurately capture MVPA and whether they are appropriate for sedentary behaviour research questions is unknown. For example, it has been suggested that wear time has a disproportionate impact upon estimates of sedentary time compared with MVPA [44]. Teasing out which sedentary behaviours beyond screen time are associated with negative health outcomes represents an important area for future research

The sedentary behaviours that are of known public health concern in children and youth (eg., excessive levels of screen time) typically last for extended periods of time (i.e., up to several hours at once). This reality was the motivation behind the way prolonged bouts of sedentary time were defined in the present analysis. Had we used a strict definition of what ended about (i.e., any transition out of sedentary) then our longest bout length would have been very short (e.g, 10 minutes) and thus not representative of one of the key sedentary behaviours that we were interested in capturing. The allowance of a modest amount of light intensity movement within the prolonged sedentary bouts was therefore purposeful and allowed much longer bout lengths to be 
examined (up to 2 hours). Number of breaks per day, also assessed in this analysis, is an important aspect of sedentary behaviour patterns. Given that we and others [1] have not consistently observed significant associations between number of breaks per day and health risk, it is important to look at alternative pattern variables such as prolonged bouts. Further, the extension of bout length in the present analysis was important to build off the only other published work that examined prolonged bouts up to 30 minutes in children and youth [1].

It is possible that the true health effect of sedentary time is attenuated by limitations with the data and analysis. Possible limitations that dilute the possibility of observing a true relationship include: i) the cross-sectional nature of the data, ii) non-response bias, iii) the possibility that the findings in 11-14 year olds boys reflect Type 1 error. As described in the methodology, the nonresponse bias is adjusted for in the data. We attempted to minimize the likelihood of Type 1 errors in the regression analyses by adjusting the p-value for significance from .05 to .006 . Other limitations include the lack of ability to confirm precisely when children were finished school. We examined the period after $3 \mathrm{pm}$ on weekdays [45] based on the assumption that most kids would finish school sometime between 2-4 pm. Accelerometers are limited in their ability to capture postural changes (i.e., cannot differentiate between sitting and standing) and are therefore limited in their ability to measure sedentary before as well as other tools which encompass an inclinometer in addition to an accelerometer. No significant associations were observed between sedentary time variables and blood pressure or nonHDL cholesterol and this may be due to it likely being more difficult to detect meaningful differences in biomarkers in children and youth than adults because younger people are more distal to pathophysiological developments. A similar examination on a population of high-risk children (e.g., overweight or obese or with a family history of cardio-metabolic disease) may lead to different findings as these children would be more likely to exhibit abnormalities in blood markers and blood pressure. Finally, examination of interaction and confounding effects was limited because the number of variables (including interaction terms) that can be tested within the CHMS data set is limited by the available de grees of freedom.

\section{Conclusions}

Sedentary time accumulated during the after school period was associated with BMI and waist circumference, independent of MVPA, in boys aged 11 to 14 years. No sedentary behaviour variables were independently associated with any health markers in older or younger boys or in girls of any age. Future studies should consider examining more comprehensive sedentary time pattern variables when attempting to elucidate the relationships between sedentary time and health risk in children and youth.

\section{Additional files}

Additional fille 1: Table $\mathbf{5 1}$, Associations between sedentary tims Additional file 1: Table 51. Associations between sedertary tim
variables and booy mass index, presented by sea and age groups Additional file 2: Table S2. Associations between sedenary time variabiles and wakt cheumderence, presented by sex and age group Additional file 3: Table S3. Assoclations between sedertary time varisties and systolic blood pressure, presented by sex and sge groups Additional file 4: Table S4, Associations between sedertary time uariatiles and diastolic blood pressure, presented by sex and age groups Additional file 5: Table 55. Associations between sedenary time variables and non-HDC cholesterol presented by sex and age groups

\section{Competing interests}

The authors declare thas they have no competing imerests

Authors' contributions

ACC conceived the manuscipc formed the research ream direcied the analysis led the writing DG was imolved in the conoeption the manuscriot completed the andysis and contrbuted to the werting. II contributed to the writing and provided critical rewiow of the analysk. SLW was imolived in the conception of the manuscrips, contributed to the writing and purvided critical review of the anslyss and writing TIS, WC and MST contributed to the writing and provided critical review of the writing. All authars read and spproved the finsal manuscript.

Acknowledgements

The authors would liko to thank the participares of the Canadian Heater Messures Survey as wedl as all staff at Statiktics Canada imvolved in the. aperations of the survey.

Author details

'Health Andysis Ohision Statistics Canoda, Detama ON Conada. "Ovildren's Hospital of Eastem Oneario Alesearch Instiuse. Ortewa. ON. Canada 'Schoel of Knesioboy and Hearth Studiex, Quen's Uniwersity, Kingston oN Caras Department of Commurity Hoalth and Epidomiology, Qusen's Lhiversty. Ningstan, ON, Canada.

Heceived 25 October 2012 Accepted: 22 February 2013 Published: 7 March 2013

Rieferences

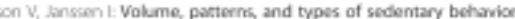
and caediometabolic health in children and adolescents a cross.

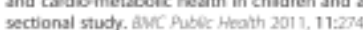

Mork AE trogen l: Aelotionship between acreen time and metabolc

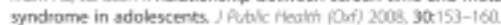

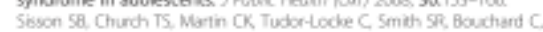
Eamest CP. Parkinen T, Nomenon fy, Karmarge PT. Profles of sedent behiwior in chiblen ind adoleccents: The US Nimional Heath and

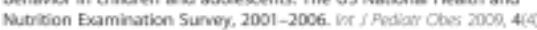
353-359.

4. Tremblyy MS, Lebirnc AG, Who ME, Ssunders T, Laiouche R, Colley RC, Galdfield G, Connor Garber SS Systematic review of sedertary behoviour and health indicators in school-aged children and youth hit I bohor Nutr Pfys Ad 2011, 8:89

5. Sederkary betheviour peseanch Network Leter to the editor: standarsized use of the lerms "sedernary" and "setemary behowiours". Agel Fhos" Mis Merob 2012, 37131540-542

6. Adamo KB, Fince SA, Ticco AC, Connor-Gorter 5, Themblay MS: A comparison of indrect verges direct measures for assessing physical 
activity in the pedistric population a systematic review. int 5 Pediot Obe $2000,4: 172-27$

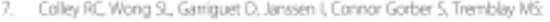

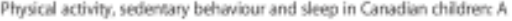
comparisen between parent-report and accolencmenty messurs and the retalive association with health cutcomes. Heath Rep 2012, 23:27:1-9?

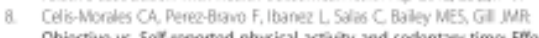
Objective us ser-eqpented physial activey and sedentary times Eflects of measuremens method on retationshiox with risk biomarkers. Flos one 2012, 7506036345

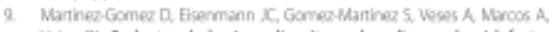
Weiga OL: Sedentary behavior, adiposity, and candiovascular risk faccoer

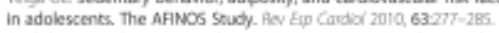

10. Sandirha LB, Andersen LB, Andersen SA, Quiteria AL, Omols R, Froberg $K$, Andersen SA, Fiddoch C Q Quiteria ML, Elefind U Objectively measured time spent sedentary $\mathrm{s}$ associated with insuln resstance independent of overall and central body fot in 9 to 10 -year-old Portuguese childien Dabetes Care $20083103069-575$

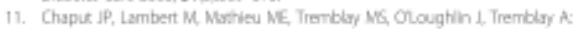
Physical activity vs. sedentary time independent associstions with

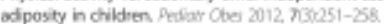

12. Chinapew MiM, Midrim M, Alterburg TM, Singh AS, Kovass E, Mohor $D$, Arug tobjective and seff-rated sederesry time and indicators of mesabolic health in Outch and Hungarion 10-12 yeas obids: The ENERCY project. Plos 0 ne 201275003665 ?

13. Elosund U, Lasa 1, Sherar LA, Esliger DW. Giew P. Cocper A, for the

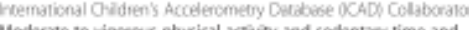
Maderale va vigoras physical active and sedentary time and

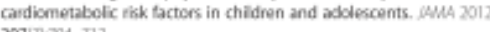

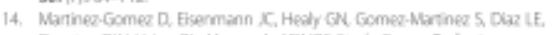
Dinstan DWV Veiga OC, Marcos A, AANOS Study Gioup: Sedemary Dehavioss and emerging carction

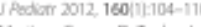

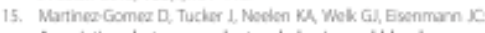
Associstions between sedentary behavior and blood pressue in young

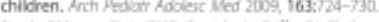

16. Soede AM, wan Sijs EMF, Considy A Giffin S1, Boelund U: Targeting sedentary time or moderabe- and vigorous -imensity activity. independent relstione with adposiry in a popustion-based sample of 10-y-old British chidren Am J Con Nutr 2009, 90:1165-1192.

17. Heak GN, Dunstan DW, Salmon I, Cerin E, Shyw E, Zmmet P, Omen Ne Breaks in sedentary time - Beneficial associations with metabolic riak

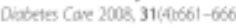

18. Attbin Al, Gorely T, Bidde SI, Marshal SJ, Cameron Nc Critical hours physical activity and sedentary behaviour of adolescents after school. Peofict Ever Soj 2006. 200446-456

19. Fariclough SL, Bucher ZKS Stutton G. Whok-day and segmented-dry physical activity varisblity of nort-mest Endend school childen Prev Med $20097,44 \times 21-42$.

20. Haringlon CM, Dowd WP, Bcourke AK, Dornely NE Cross-sectional andysis of levels and patiens of objectivety measred sedentior time in

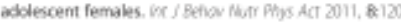

21. Doy B. Landoo R Trembloy MS, Knoppers B-M. Canadian Hedith Measures Sarvey: Ethical legal and social bsues, Heath fiep 2007, 18:37-51.

22. Tremblyy MG, Wolfson M Connor Gorter S. Canadian Hedth Measures Sarvey: background, rationsle and overview. Heabh few 2009 , $18 \mathrm{cuppl}$ ? 720

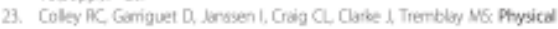
axtiviny of Comadian chidren and youth: Accelesometer resuls from the 2007 to 2000 Canadian Health Mersures Survey. Heath fieg 201 ) $2271215-23$

24. Colley AC, Gober SC. Tremblay MG Oailty canerol and data reduction procedues for accolerometry-derived measures of physical activing.

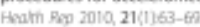

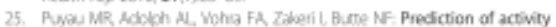

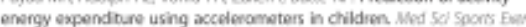
2004 3619:16:5-1631.

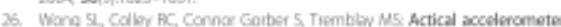
sedentary activing threshold for aduits. J Phy Act Heath 2011,8557-591.

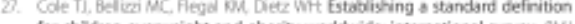

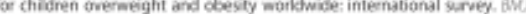
$2000,320: 1240$

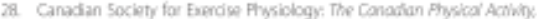

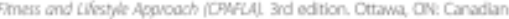
Sociesy for Evercise Physiologry 2004

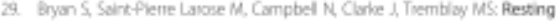
blood pressure and heart rate measurement in the Cangdian thegith

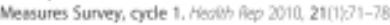

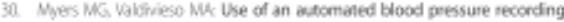
device, the BpTru, to reduce the "while cast effect" in routine practice

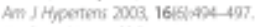

31. Gardiner CD, Whileby MA, Foromann SP. Popdation frequency of non high-density lipocrosein cholesterol (Thind National Hesth and Nutrition

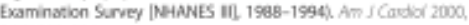
50:31299-104

32 Li l, Josti D, Sempos CT: Non-high-density lipoprotein cholesteol and candiovasodar risk factors among adolescents with ind witheut

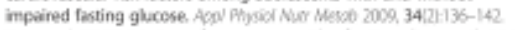

33. Karmangk. PT: Waist ciroumfermoe pescencles for Canadian youth 11-18y of age. Fur / Cho Nerr 2004 5897:1011-1015.

34. Fas INK, WU CFI, Yue K Some recemt work on resampling methods for

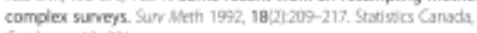
Gatalogue 12-001.

35. Aust KF, Ros NWK Variance estimetion for complex surveys using reslication techriques. 5lox Methods Med Pes 1996, 5:281-310.

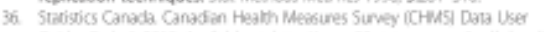

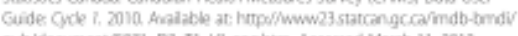
pubstocumene/5071_D2_T1_V1-enghtrm. Acossed Makch 11, 2013

37. Tremblyy MS, Colley $\pi$, Saunders TL, Healy GM, Owen N: Physiblogical and

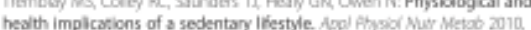
35:225-790.

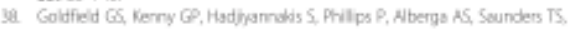
Trembloy MS. Malodm I. Prudhomme D, Gougeon R. Sigd RU: Video game

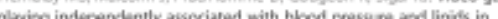

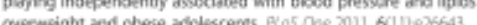

Granich $l$ Fosenberg M Knuman MW. Timperto A. Indwidid, secial, and

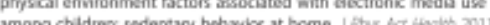

40. Marshal SI, Govety T, Biddle St. A descriptive epidemiology of socen-

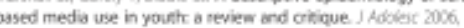
29131333-3e9

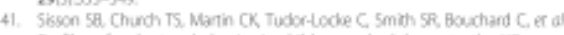
Protiles of sedentary behwior in children and adoloscencs the US National Heatoh and Methon Eam

42. Currie C, Znoutt C, Morgen A, Currie D, de Loaxe M, Paberts C, et al (Eds) Socis cheteminants of heath and werl being among young perple. Heath

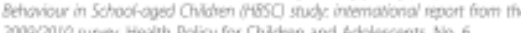

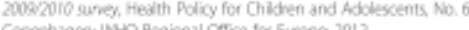
coperhagen: WHO Regmat Chice tor Eurcper 2012

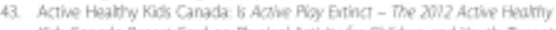

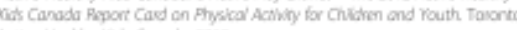

44. Tudor-Lode C, Jonson WO, Katzmasyk PT: U.S. Population profile of time stamped accelerometer cutputs: Impsct of wear time.J thys Act thath 2011, 8t993-698

45. Faindough SI. Beighte A, Exwin H. Fodgers ND: School diay segmented phtysical activity pattems of high and low active children sMC A, Heath 2012,12406

Soi:10.1186/1471-2458-13-20 Che this article as: Coley et $\alpha$ : The association between sccelerometer measured pattems of sedentary time and hoalth risk in ckildren and youth: results from the Canadian Health Measures Survey, aMC Puth 


\section{Appendix D: Publications during PhD Studies}

\subsection{Published and Accepted Manuscripts}

1. Saunders TJ, Chaput JP, Goldfield GS, Colley RC, Kenny GP, Doucet E, Tremblay MS. Prolonged sitting and markers of cardiometabolic disease risk in children and youth: A randomized crossover study. Metabolism, In Press. $\mathrm{IF}=2.772$.

2. Larouche R, Saunders TJ, Colley RC, Faulkner G, Tremblay MS. Associations between active school transport and physical activity, body composition and cardiovascular fitness: a systematic review of 57 studies. Journal of Physical Activity and Health. In Press.

3. Saunders TJ, Tremblay MS, Després JP, Bouchard C, Tremblay A, Chaput JP. Sedentary behaviour, visceral fat accumulation and cardiometabolic risk in adults: a 6-year longitudinal study from the Quebec Family Study. PLOS ONE, 8(1): e54225, 2013. IF = 4.411.

4. Goldfield G., Saunders T.J., Kenny G.P., Hadjiyannakis S., Phillips P., Alberga A.S., Tremblay M.S., Malcolm J., Prud'homme D., Gougeon R., Sigal R.J. TV Viewing and Diabetes Risk Factors in Overweight and Obese Adolescents. American Journal of Preventive Medicine, 44(4 Suppl 4):S364-70, 2013. $\mathrm{IF}=5.128$.

5. Thivel D, Aucouturier J, Doucet E, Saunders TJ, Chaput JP. Daily energy balance in children and adolescents: does energy expenditure predict subsequent energy intake? Appetite, 60(1):58-64, 2013. IF=3.022.

6. Thivel D, Saunders TJ, Chaput JP. Physical activity in children and youth may have a greater impact on energy intake than energy expenditure. Journal of Nutrition Education and Behavior, 45(1):e1, 2013.

7. Colley RC, Garriguet D, Janssen I, Wong SL, Saunders TJ, Carson V, Tremblay MS. The association between accelerometer-measured patterns of sedentary time and health risk in children and youth: a cross-sectional study. BMC Public Health, 13:200, 2013.

8. Chaput JP, Saunders TJ, Mathieu MÈ, Henderson M, Tremblay MS, O'Loughlin $\mathrm{J}$, Tremblay A. Combined associations between moderate to vigorous physical activity and sedentary behaviour with cardiometabolic risk factors in children. Appl Physiol Nutr Metab, 38(5):477-83, 2013. IF=2.401.

9. Saunders TJ, Chaput JP. Is obesity prevention as simple as turning off the TV and having a nap? British J Nutrition, 108(5):946-7, 2012. IF=3.013. 
10. Saunders TJ, RLarouche, RC Colley, MS Tremblay. Acute Sedentary Behavior and Markers of Cardiometabolic Risk: A Systematic Review of Intervention Studies. Journal of Nutrition and Metabolism, 2012:712435.

11. Saunders TJ, A Palobella, KA McGuire, P Janiszewski, JP Després , R Ross.Acute exercise increases adiponectin levels in abdominally obese men. Journal of Nutrition and Metabolism, 2012:148729.

12. Sedentary Behaviour Research Network (Barnes J, Behrens TK, Benden ME, Biddle S, Bond D, Brassard P, Brown H, Carr L, Chaput JP, Christian H, Colley RC, Duggan M, Dunstan D, Ekelund U, Esliger D, Ferraro Z, Freedhoff Y, Galaviz K, Gardiner P, Goldfield G, Haskell WL, Liguori G, Healy G, Herman KM, Hinckson E, Larouche R, Leblanc A, Levine J, Maeda H, McCall M, McCubbin W, McGuire A, Onywera V, Owen N, Peterson M, Prince S, Ramirez E, Ridgers N, Routen A, Rowlands A, Saunders TJ, Schuna JM, Sherar L, Spruijt-Metz D, Taylor B, Tremblay MS, Tucker J, Widndaele K, Wilson J, Woodruff S). Standardized use of the terms "sedentary" and "sedentary behaviours". Applied Physiology, Nutrition and Metabolism, 37(3):540-2, 2012. IF $=2.401$

13. Chaput J.P., Saunders T.J. Bioenergetics of Obesity: Is Fat Gain a Problem or a Solution? Bioenergetics: Open Access, 2012.

14. Larouche R, Saunders TJ. Can active school transport prevent overweight and obesity in children and youth? Health Science Inquiry, 2012; 3:64-65.

15. Saunders TJ. Potential Contributors to the Canadian Pediatric Obesity Epidemic. ISRN Pediatrics, 2011.

16. Goldfield G.S., Kenny G.P., Hadjiyannakis S., Phillips P., Alberga A.S., Saunders T.J., Tremblay M.S., Malcolm J., Prud'homme D., Gougeon R., Sigal R.J. Video Game Playing Is Independently Associated with Blood Pressure and Lipids in Overweight and Obese Adolescents PLoS ONE, 2011;6(11):e26643. IF $=4.411$.

17. Tremblay MS, LeBlanc A.G., Kho M.E., Saunders T.J., Larouche R., Colley R.C., Goldfield G., Connor Gorber S. Systematic review of sedentary behaviour and health indicators in school-aged children and youth. International Journal of Behavioural Nutrition and Physical Activity8:98, 2011. IF $=3.830$.

18. Saunders T.J., Prince S.A., Tremblay M.S. Clustering of children's activity behaviour: the use of self-report versus direct measures. International Journal of Behavioural Nutrition and Physical Activity 8:48, 2011. IF =3.830.

19. Tremblay, M.S., Colley, R., Saunders, T.J., Healy, G.N., \& Owen, N. Physiological and Health Implications of a Sedentary Lifestyle. Applied Physiology, Nutrition, \& Metabolism. 35 (6): 725-40, 2010. IF $=2.401$. 
20. Bullimore, S.R., Saunders, T.J., Herzog, W., \& MacIntosh, B.R. Calculation of muscle maximal shortening velocity by extrapolation of the force-velocity relationship: afterloaded versus isotonic release contractions. Canadian Journal of Physiology and Pharmacology. 88 (10): 937-948, 2010. IF $=1.953$.

21. Church, T., Earnest, C., Thompson, A., Priest, E., Rodarte, R., Saunders, T.J., Ross, R., \& Blair, S. Exercise Without Weight Loss Does Not Reduce CReactive Protein: The INFLAME Study. Medicine \& Science in Sports \& Exercise. 42 (4): 708-716, 2010. IF $=3.710$.

22. Janiszewski, P.M., Saunders, T.J.\& Ross, R. Association between breast volume, cardiometabolic risk factors and fat distribution in premenopausal women. Obesity. 18 (6): 1183-7, 2010. IF = 4.284.

23. Saunders, T.J., Davidson, L.E., Janiszewski, P.M., Despres, J.-P., Hudson, R., \& Ross, R. Associations of the limb fat to trunk fat ratio with markers of cardiometabolic risk in elderly men and women. Journals of Gerontology Series: Medical Sciences. 64A (10): 1066-1070, 2009. IF $=4.598$.

24. Kuk, J.L., Saunders, T.J., Davidson, L.E., \& Ross, R. Age-related Changes in Total and Regional Fat Distribution. Ageing Research Reviews. 8 (4): 339-348, 2009. IF $=6.383$. 


\subsection{Published Abstracts}

1. Saunders TJ, Tremblay MS, Després JP, Bouchard C, Tremblay A, Chaput JP. Sedentary behaviour, visceral fat accumulation and cardiometabolic risk in adults: a 6-year longitudinal study from the Quebec Family Study. Medicine and Science in Sports \& Exercise, 45(5): S84, 2013.

2. Saunders, T.J., Larouche, R., Colley, R.C., Tremblay M.S. Acute sedentary behaviour and markers of cardiometabolic risk: a systematic review of intervention studies. Medicine and Science in Sports \& Exercise, 44: S883, 2012.

3. Saunders T.J., Palombella A., McGuire, K.A. Janiszewski, P.M., Després, J.P., Ross, R. Acute exercise increases adiponectin levels in abdominally obese men.Applied Physiology, Nutrition and Metabolism, 36: S350, 2011.

4. Tremblay, M.S., Leblanc, A.G., Colley, R., Goldfield, G., Saunders, T.\& Larouche, R. Systematic review of the relationship between sedentary behavior and health indicators in school-aged children and youth. Applied Physiology, Nutrition, and Metabolism, 35: S103, 2010.

5. Saunders, T.J., Palombella, A., McGuire, K.A., Janiszewski, P.M., \& Ross, R. Temporal changes in plasma triglyceride levels following acute exercise in abdominally obese men: effect of exercise intensity. Applied Physiology, Nutrition, and Metabolism, 34: S82, 2009. 